\title{
LECTURES ON THE THEORY OF RECIPROCANTS.
}

[American Journal of Mathematics, viII. (1886), pp. 196-260; Ix. pp. 1-37, 113-161, 297-352 ; x. pp. 1-16. Delivered in Oxford, 1886.]

THE lectures here reproduced were delivered, or are still in the course of delivery, before a class of graduates and scholars in the University of Ox ord during the present year. They are to be regarded as easy lessons in the new Theory of Reciprocants of which an outline will be found in Nature for January 7, which contains a report of a Public Lecture on the subject delivered before the University of Oxford in December of the preceding year.

They are designed as a practical introduction to an enlarged theory of Algebraical Forms, and as such are not constructed with the rigorous adhesion to logical order which might be properly expected in a systematic treatise. The object of the lecturer was to rouse an interest in the subject, and in pursuit of this end he has not hesitated to state many results, by way of anticipation, which might, with stricter regard to method, have followed at a later period in the course.

There will be found also occasional repetitions and intercalations of allied topics which are to be justified by the same plea, and also by the fact that the lectures were not composed in their entirety previous to delivery, but gradually evolved and written between one lecture and another in the way that seemed most likely to the lecturer to secure the atterition of his auditors.

Since the delivery of his public lecture in December last, papers have been contributed on the subject to the Proceedings of the Mathematical Society of London by Messrs Hammond, MacMahon, Elliott, Leudesdorf and Rogers, and one to the Comptes Rendus de l'Institut by M. George Perrin. It may therefore be inferred that the lectures have not altogether failed in attaining the desired end of drawing attention to a subject which, in the opinion of the lecturer, constitutes a very considerable extension of the previous limits of algebraical science. 


\section{LECTURE I.}

A new world of Algebraical forms, susceptible of important geometrical applications, has recently come into existence, of which I gave a general account in a public lecture at the end of last term. I propose in the following brief course to go more fully into the subject and lay down the leading principles of the theory so far as they are at present known to me. The parallelism between the theory of what may be called pure reciprocants and that of invariants is so remarkable that it will be frequently expedient to pass from one theory to the other or to treat the two simultaneously. It may be as well therefore at once to give notice that the term invariant will hereafter be applied alike to invariants ordinarily so called and to those more general algebraical forms which have been termed sources of covariants, differentiants, seminvariants, or subinvariants. A form which is an invariant in the old sense will be termed, when necessary to specify it, a satisfied invariant, an expression which the chemico-graphical representation of invariants or covariants will serve to explain and justify.

In an elueidatory course of lectures such as the present, it will be advisable to follow a freer order of treatment than would be suitable to the presentation of it in a systematic memoir. My object is to make the theory known, to excite curiosity regarding it, and to invite co-operation in the task of its development.

By way of introduction to the subject, let us begin with an investigation of the properties of a differential expression involving only the first, second and third differential coefficients of either of two variables in respect to the other. For this purpose let us consider not what I have called the Schwarzian itself, which is an integral rational function of these three quantities, but the fractional expression

$$
\frac{\frac{d^{3} y}{d x^{3}}}{\frac{d y}{d x}}-\frac{3}{2}\left(\frac{\frac{d^{2} y}{d x^{2}}}{\frac{d y}{d x}}\right)^{2}
$$

which becomes the Schwarzian when cleared of fractions, and which after Cayley we may call the Schwarzian Derivative and denote by

$$
(y, x) \text {; }
$$

$(x, y)$ will then of course mean

$$
\frac{\frac{d^{3} x}{d y^{3}}}{\frac{d x}{d y}}-\frac{3}{2}\left(\frac{\frac{d^{2} x}{d y^{2}}}{\frac{d x}{d y}}\right)^{2}
$$


It is easy to establish the identical equation

$$
(y, x)=-\left(\frac{d y}{d x}\right)^{2}(x, y)
$$

Using for brevity $y^{\prime}, y^{\prime \prime}, y^{\prime \prime \prime}$ to denote, as usual,

and $x_{,}, x_{\prime \prime}, x_{\prime \prime}$, to denote

$$
\frac{d y}{d x}, \frac{d^{2} y}{d x^{2}}, \frac{d^{3} y}{d x^{3}}
$$

$$
\frac{d x}{d y}, \frac{d^{2} x}{d y^{2}}, \frac{d^{3} x}{d y^{3}},
$$

respectively, the relation to be verified is

$$
\frac{2 y^{\prime} y^{\prime \prime \prime}-3 y^{\prime \prime 2}}{y^{\prime 2}}=-y^{\prime 2} \cdot \frac{2 x_{1} x_{1 \prime \prime}-3 x_{\prime \prime}{ }^{2}}{x_{!}^{2}} \text {. }
$$

Now,

$$
\begin{aligned}
& x_{1}=\frac{1}{y^{\prime}} \\
& x_{/ 1}=\frac{d}{d y}\left(x_{\prime}\right)=\frac{1}{y^{\prime}} \cdot \frac{d}{d x}\left(\frac{1}{y^{\prime}}\right)=-\frac{y^{\prime \prime}}{y^{\prime 3}},
\end{aligned}
$$

and

$$
x_{11 \prime}=\frac{d}{d y}\left(x_{1 \prime}\right)=\frac{1}{y^{\prime}} \cdot \frac{d}{d x}\left(-\frac{y^{\prime \prime}}{y^{\prime 3}}\right)=-\frac{y^{\prime \prime \prime}}{y^{\prime 4}}+\frac{3 y^{\prime 2}}{y^{\prime 5}} .
$$

Whence we obtain

and the truth of (1) is manifest.

$$
\begin{aligned}
2 x_{1} x_{1 \prime}-3 x_{\prime \prime}{ }^{2} & =\left(-\frac{2 y^{\prime \prime \prime}}{y^{\prime 5}}+\frac{6 y^{\prime \prime 2}}{y^{\prime 6}}\right)-\frac{3 y^{\prime \prime 2}}{y^{\prime 6}} \\
& =-\frac{1}{y^{\prime 6}}\left(2 y^{\prime} y^{\prime \prime \prime}-3 y^{\prime \prime 2}\right)
\end{aligned}
$$

This may be put under the form

$$
\frac{2 y^{\prime} y^{\prime \prime \prime}-3 y^{\prime \prime 2}}{y^{\prime 3}}=-\frac{2 x_{1} x_{1 \prime \prime}-3 x_{1 \prime}{ }^{2}}{x_{1}^{3}}
$$

showing that a certain function of the first, second and third derivatives of one variable in respect to another remains unaltered, save as to algebraical sign, when the variables are interchanged. An example of a similar kind with which we are all familiar is presented by the well-known function $\frac{d^{2} y}{d x^{2}} \div\left(\frac{d y}{d x}\right)^{\frac{3}{2}}$, which is equal to $-\frac{d^{2} x}{d y^{2}} \div\left(\frac{d x}{d y}\right)^{\frac{3}{2}}$

We are thus led to inquire whether there may not be an infinite number of algebraical functions of differential derivatives which possess a similar property, and by prosecuting this inquiry to lay the foundations of the theory of Reciprocation or Reciprocants.

Having regard to the fact that the present theory originated in that of the Schwarzian Derivative, I shall proceed to demonstrate, although this is s. IV. 
not strictly necessary for the theory of Reciprocants, the remarkable identity

$$
(y, x)-(z, x)=\left(\frac{d z}{d x}\right)^{2} \cdot(y, z)
$$

This identical relation is the fundamental property of Schwarzians, and from it every other proposition concerning their form is an immediate deduction.

In the following proof*, $y$ and $z$ are regarded as two given functions of any variable $t$, and $x$ as a variable function of the same: so that $y$ and $z$ are functions of $x$ for any given function that $x$ is of $t$.

It will be seen that

$$
\{(y, x)-(z, x)\}\left(\frac{d x}{d z}\right)^{2}
$$

remains unaltered by any infinitesimal variation $\theta$ of $x$, that is, when $x$ becomes $x+\epsilon \phi(x), \epsilon$ being an infinitesimal constant and $\phi(x)$ an arbitrary finite function of $x$.

For brevity, let accents denote differential derivation in regard to $x$, and let any function of $x$ enclosed in a square parenthesis signify the augmented value of that function when $x$ becomes $x+\theta$. In calculating such augmented values, since we suppose that $\theta=\epsilon \phi(x)$, it is clear that $\theta, \theta^{\prime}, \theta^{\prime \prime} \ldots$ are each of them infinitesimals of the first order, and consequently that all products, and all powers higher than the first of these quantities, may be neglected.

We have therefore

$$
\begin{aligned}
& {\left[y^{\prime}\right]=\frac{d y}{d x+d \theta}=\frac{y^{\prime}}{1+\theta^{\prime}}=y^{\prime}-\theta^{\prime} y^{\prime}} \\
& {\left[\begin{array}{rl}
\left.y^{\prime \prime}\right] & =\frac{d\left[y^{\prime}\right]}{d x+d \theta}=\frac{\frac{d}{d x}\left(y^{\prime}-\theta^{\prime} y^{\prime}\right)}{1+\theta^{\prime}}=\frac{y^{\prime \prime}\left(1-\theta^{\prime}\right)-\theta^{\prime \prime} y^{\prime}}{1+\theta^{\prime}} \\
& =y^{\prime \prime}-2 \theta^{\prime} y^{\prime \prime}-\theta^{\prime \prime} y^{\prime}
\end{array}\right.} \\
& {\left[\begin{array}{c}
\left.y^{\prime \prime \prime}\right]=\frac{d\left[y^{\prime \prime}\right]}{d x+d \theta}=\frac{\frac{d}{d x}\left(y^{\prime \prime}-2 \theta^{\prime} y^{\prime \prime}\right)-\theta^{\prime \prime} y^{\prime}}{1+\theta^{\prime}}=\frac{y^{\prime \prime \prime}\left(1-2 \theta^{\prime}\right)-3 \theta^{\prime \prime} y^{\prime \prime}-\theta^{\prime \prime \prime} y^{\prime}}{1+\theta^{\prime}} \\
=y^{\prime \prime \prime}-3 \theta^{\prime} y^{\prime \prime \prime}-3 \theta^{\prime \prime} y^{\prime \prime}-\theta^{\prime \prime \prime} y^{\prime}
\end{array}\right.} \\
& \begin{array}{c}
\quad\left[y^{\prime} y^{\prime \prime \prime}\right]=y^{\prime} y^{\prime \prime \prime}-4 \theta^{\prime} y^{\prime} y^{\prime \prime \prime}-3 \theta^{\prime \prime} y^{\prime} y^{\prime \prime}-\theta^{\prime \prime \prime} y^{\prime 2} \\
\frac{3}{2}\left[y^{\prime \prime 2}\right]=\frac{3}{2} y^{\prime \prime 2}-6 \theta^{\prime} y^{\prime \prime 2}-3 \theta^{\prime \prime} y^{\prime} y^{\prime \prime} \\
{\left[y^{\prime 2}\right]=y^{\prime 2}-2 \theta^{\prime} y^{\prime 2} .}
\end{array}
\end{aligned}
$$

And since by definition

$$
(y, x)=\frac{y^{\prime} y^{\prime \prime \prime}-\frac{3}{2} y^{\prime \prime 2}}{y^{\prime 2}}
$$

* As originally given in the Messenger of Mathematics, Vol. xv., this was defaced by so many errata as to render expedient its reproduction in a corrected form. 
we readily obtain

$$
[(y, x)]=\frac{(y, x)}{1-2 \theta^{\prime}}-4 \theta^{\prime}(y, x)-\theta^{\prime \prime \prime}=(y, x)\left(1-2 \theta^{\prime}\right)-\theta^{\prime \prime \prime} .
$$

So also

$$
[(z, x)]=(z, x)\left(1-2 \theta^{\prime}\right)-\theta^{\prime \prime \prime} .
$$

Whence by subtraction

$$
[(y, x)-(z, x)]=\left(1-2 \theta^{\prime}\right)\{(y, x)-(z, x)\} .
$$

Dividing the left-hand side of this by $\left[z^{\prime 2}\right]$, and the right-hand side by $z^{\prime 2}\left(1-2 \theta^{\prime}\right)$ which is the equivalent of $\left[z^{\prime 2}\right]$, our final result is

$$
\left[\frac{(y, x)-(z, x)}{z^{\prime 2}}\right]=\frac{(y, x)-(z, x)}{z^{\prime 2}} .
$$

Thus, then, we have seen that the expression

$$
\frac{(y, x)-(z, x)}{\left(\frac{d z}{d x}\right)^{2}}
$$

does not vary when $x$ receives an infinitesimal variation $\epsilon \phi(x)$, from which it follows, by the general principle of successive continuous accumulation, that the same will be true when $x$ undergoes any finite arbitrary variation, and consequently this expression has a value which is independent of the form of $x$ regarded as a function of $t$; it will, of course, be remembered that $y$ and $z$ are supposed to be invariable functions of $t$. Let $x$ become $z$, then $(y, x)$ becomes $(y, z)$, while at the same time $(z, x)$ vanishes and $\frac{d z}{d x}$ becomes unity: so that we obtain

$$
\frac{(y, x)-(z, x)}{\left(\frac{d z}{d x}\right)^{2}}=(y, z) \text {. }
$$

Hence, whatever function $x$ may be of $t$,

$$
(y, x)-(z, x)=\left(\frac{d z}{d x}\right)^{2} \cdot(y, z)
$$

To this fundamental proposition the equation marked (1), itself the important point in regard to the Theory of Reciprocants, is an immediate corollary. For if in (2) we interchange $y$ and $z$, it becomes

$$
(z, x)-(y, x)=\left(\frac{d y}{d x}\right)^{2} \cdot(z, y)
$$

and now, making $x=z$, we have

$$
-(y, z)=\left(\frac{d y}{d z}\right)^{2} \cdot(z, y)
$$

which is the same as (1), except that $z$ occupies the place of $x$. 
But (1) may be obtained more immediately from (2) by substituting in it $x$ for $y$ and $y$ for $z$, leaving $x$ unaltered; when it becomes

$$
-(y, x)=\left(\frac{d y}{d x}\right)^{2} \cdot(x, y)
$$

This is equivalent to saying that

$$
2 y^{\prime} y^{\prime \prime \prime}-3 y^{\prime \prime 2}=-y^{\prime 6}\left(2 x, x_{\prime \prime \prime}-3 x_{\prime \prime}{ }^{2}\right) \text {, }
$$

a verification of which has been given already.

Observe that $\frac{y^{\prime} y^{\prime \prime \prime}-\frac{3}{2} y^{\prime \prime 2}}{y^{\prime 2}}$ or $(y, x)$ contains $\left(\frac{d y}{d x}\right)^{2}$ in its denominator and $(x, y)$ contains $\left(\frac{d x}{d y}\right)^{2}$ in its denominator, which is the same as $\left(\frac{d y}{d x}\right)^{2}$ in the numerator. Thus it is that the square of $\frac{d y}{d x}$ enters three times.

Let me insist for a moment on the import of the fact brought to light in the course of this investigation, that $\frac{(y, x)-(z, x)}{\left(\frac{d z}{d x}\right)^{2}}$ is invariable when $x, y$ and $z$ being regarded as functions of $t, x$ alters its form, but $y$ and $z$ retain theirs. Of course we might write $\left(\frac{d y}{d x}\right)^{2}$ in the denominator instead of $\left(\frac{d z}{d x}\right)^{2}$, and then make the same affirmation as before; as will be evident if we only remember that by hypothesis $y$ and $z$ are both of them constant functions of $t$, and that therefore $\left(\frac{d z}{d y}\right)^{2}$ must also be so. This is tantamount to saying that when the same conditions are fulfilled $\{(y, x)-(z, x)\}(d x)^{2}$ is invariable, that is, that when $x$ becomes $X$ in virtue of any substitution (including a homographic one) impressed upon it,

$$
\{(y, x)-(z, x)\}(d x)^{2}=\{(y, X)-(z, X)\}(d X)^{2},
$$

and thus we see that when $x$ becomes $X$,

$$
(y, x)-(z, x)
$$

remains unaltered except that it takes to itself the factor $\left(\frac{d X}{d x}\right)^{2}$ which depends solely on the particular substitution impressed on $x$.

If

$$
y=f(x), z=\phi(x), \text { and } X=\omega(x),
$$

our formula becomes

$$
\{(f x, x)-(\phi x, x)\}(d x)^{2}=\left\{\left(f \omega^{-1} X, X\right)-\left(\phi \omega^{-1} X, X\right)\right\}(d X)^{2},
$$

so that, speaking of Quantics and Covariants with respect to a single variable $x,(f x, x)-(\phi x, x)$ is to all intents and purposes a Covariant to the simultaneous forms $f(x)$ and $\phi(x)$, in a sense comprehending but far transcending that in which the term is ordinarily employed; for it remains a persistent 
factor of its altered self when for $x$ any arbitrary function of $x$ is substituted, the new factor taken on depending wholly and solely on the particular substitution impressed upon $x$. In the ordinary theory of invariants, the substitution impressed is limited to be homographic; in this case it is absolutely general. We might, moreover, add as a corollary that if $(y, x)$, $(z, x),(u, x) \ldots$ are regarded as roots of any Binary Quantic, every invariant of that Binary Quantic is a covariant in the extended sense in which the word has just been used, in respect to the system of simultaneous forms $f(x), \phi(x), \psi(x) \ldots$ For every such invariant will be a function of

$$
(y, x)-(z, x), \quad(y, x)-(u, x), \quad(z, x)-(u, x), \ldots
$$

and will therefore remain a persistent factor of its altered self, taking on a power of $\frac{d X}{d x}$ as its extraneous factor.

Calling $(f x, x)$ the Schwarzian Derivative of $f(x)$, our theorem may be stated in general terms as follows:

All invariants of a Binary Quantic whose roots are the Schwarzian Derivatives of a given set of functions of the same variable are Covariants (in an extended sense) of that set of functions.

The theory of the Schwarzian derivative originates in that of the linear differential equation of the second order,

$$
u^{\prime \prime}+2 P u^{\prime}+Q u=0
$$

which becomes, when we write $u=v e^{-\int P d x}$,

$$
v^{\prime \prime}+I v=0
$$

where

$$
I=Q-P^{2}-P^{\prime}
$$

Now, suppose that $u_{1}$ and $u_{2}$ are any two particular solutions of the first of these equations, and let $z$ denote their mutual ratio; so that, when $v_{1}$ and $v_{2}$ are the corresponding particular solutions of the second equation, we readily obtain

and therefore, $\quad z^{\prime}=\frac{v_{1} v_{2}^{\prime}-v_{2} v_{1}^{\prime}}{v_{1}^{2}}$.

$$
\begin{gathered}
z=\frac{u_{2}}{u_{1}}=\frac{v_{2}}{v_{1}}, \\
z^{\prime}=\frac{v_{1} v_{2}^{\prime}-v_{2} v_{1}^{\prime}}{v_{1}^{2}} .
\end{gathered}
$$

A second differentiation gives

$$
z^{\prime \prime}=\frac{v_{1} v_{2}^{\prime \prime}-v_{2} v_{1}^{\prime \prime}}{v_{1}^{2}}-\frac{2 v_{1}^{\prime}\left(v_{1} v_{2}^{\prime}-v_{2} v_{1}^{\prime}\right)}{v_{1}^{3}} .
$$

But since

$$
\frac{v_{1}^{\prime \prime}}{v_{1}}=\frac{v_{2}^{\prime \prime}}{v_{2}}=-I
$$

the first term of the expression just found vanishes identically, and we have

$$
z^{\prime \prime}=-\frac{2 v_{1}^{\prime} z^{\prime}}{v_{1}}
$$


or,

$$
v_{1}^{\prime}=-\frac{z^{\prime \prime} v_{1}}{2 z^{\prime}}
$$

Differentiating this again, we find

Hence

$$
\begin{aligned}
-2 v_{1}^{\prime \prime}= & \left(\frac{z^{\prime \prime \prime}}{z^{\prime}}-\frac{z^{\prime \prime 2}}{z^{\prime 2}}\right) v_{1}+\frac{z^{\prime \prime}}{z^{\prime}} v_{1}^{\prime} \\
= & \left(\frac{z^{\prime \prime \prime}}{z^{\prime}}-\frac{3}{2} \frac{z^{\prime 2}}{z^{\prime 2}}\right) v_{1} . \\
& \frac{z^{\prime \prime \prime}}{z^{\prime}}-\frac{3}{2} \frac{z^{\prime \prime 2}}{z^{\prime 2}}=2 I,
\end{aligned}
$$

where the left-hand side of the equation is "the Schwarzian Derivative" with $z$ written in the place of $y$.

\section{LECTURE II.}

The expression $2 y^{\prime} y^{\prime \prime \prime}-3 y^{\prime \prime}$, which we have called the Schwarzian, may be termed a reciprocant, meaning thereby that on interchanging $y^{\prime}, y^{\prime \prime}, y^{\prime \prime \prime}$ with $x_{1}, x_{1,}, x_{\prime \prime \prime}$ its form remains unaltered, save as to the acquisition of what may be called an extraneous factor, which, in the case before us, is a power of $y^{\prime}$ (with a multiplier -1). Before we proceed to consider outher examples of reciprocants it will be useful to give formulae by means of which the variables may be readily interchanged in any differential expression.

We shall write $t$ for $y^{\prime}$ and $\tau$ for its reciprocal $x_{\text {, }}$, using the letters $a, b, c, \ldots$ to denote the second, third, fourth, etc., differential derivatives of $y$ with respect to $x$, and $\alpha, \beta, \gamma, \ldots$ to denote those of $x$ with respect to $y$. The advantage of this notation will be seen in the sequel.

The values of $\alpha, \beta, \gamma, \ldots$ in terms of $t, a, b, c, \ldots$ are given by the formulae

$$
\begin{aligned}
& \alpha=-a \div t^{3} \\
& \beta=-b t+3 a^{2} \div t^{5} \\
& \gamma=-c t^{2}+10 a b t-15 a^{3} \div t^{7} \\
& \delta=-d t^{3}+\left(15 a c+10 b^{2}\right) t^{2}-105 a^{2} b t+105 a^{4} \div t^{9} \\
& \epsilon=-e t^{4}+(21 a d+35 b c) t^{3}-\left(210 a^{2} c+280 a b^{2}\right) t^{2}+1260 a^{3} b t-945 a^{5} \div t^{11},
\end{aligned}
$$

If, in these equations, we write

and

$$
\begin{aligned}
& a=1.2 . a_{0}, \quad b=1.2 .3 . a_{1}, \quad c=1.2 .3 .4 . a_{2}, \ldots \\
& \alpha=1.2 . \alpha_{0}, \quad \beta=1.2 .3 . \alpha_{1}, \quad \gamma=1.2 .3 .4 . \alpha_{2}, \ldots
\end{aligned}
$$


they become

$$
\begin{aligned}
& \alpha_{0}=-a_{0} \div t^{3} \\
& \alpha_{1}=-a_{1} t+2 a_{0}^{2} \div t^{5} \\
& \alpha_{2}=-a_{2} t^{2}+5 a_{0} a_{1} t-5 a_{0}^{3} \div t^{7} \\
& \alpha_{3}=-a_{3} t^{3}+\left(6 a_{0} a_{2}+3 a_{1}^{2}\right) t^{2}-21 a_{0}^{2} a_{1} t+14 a_{0}^{4} \div t^{9} \\
& \alpha_{4}=-a_{4} t^{4}+\left(7 a_{0} a_{3}+7 a_{1} a_{2}\right) t^{3}-\left(28 a_{0}{ }^{2} a_{2}+28 a_{1}^{2}\right) t^{2}+84 a_{0}^{3} a_{1} t-42 a_{0}^{5} \div t^{11}
\end{aligned}
$$

Any one of the formulae in either set may be deduced from the formula immediately preceding it by a simple process of differentiation.

Thus, since

$$
\beta=\frac{-b t+3 a^{2}}{t^{5}} \text { and } \frac{d}{d y}=\frac{1}{t} \cdot \frac{d}{d x},
$$

we have

$$
\frac{d \beta}{d y}=\frac{1}{t} \cdot \frac{d}{d x}\left(\frac{-b t+3 a^{2}}{t^{5}}\right)
$$

But

$$
\frac{d \beta}{d y}=\gamma \text { and } \frac{d}{d x}=a \partial_{t}+b \partial_{a}+c \partial_{b}+\ldots,
$$

so that

$$
\begin{aligned}
\gamma & =\frac{1}{t}\left(a \partial_{t}+b \partial_{a}+c \partial_{b}+\ldots\right)\left(\frac{-b t+3 a^{2}}{t^{5}}\right) \\
& =\frac{1}{t^{7}}\left(-c t^{2}+10 a b t-15 a^{3}\right) .
\end{aligned}
$$

By continually operating with $\frac{1}{t}\left(a \partial_{t}+b \partial_{a}+c \partial_{b}+\ldots\right)$ the table may be extended as far as we please, the expressions on the right-hand side being the successive values of

$$
\left\{\frac{1}{t}\left(a \partial_{t}+b \partial_{a}+c \partial_{b}+\ldots\right)\right\}^{n}\left(-\frac{a}{t^{3}}\right)
$$

found by giving to $n$ the values $0,1,2,3, \ldots$.

Precisely similar reasoning shows that, when the modified letters $a_{0}, a_{1}, a_{2}, \ldots$ are used,

$$
\begin{aligned}
& (n+2) \alpha_{n}=\frac{1}{t}\left(2 a_{0} \partial_{t}+3 a_{1} \partial_{a_{0}}+4 a_{2} \partial_{a_{1}}+\ldots\right) \alpha_{n-1} \\
& \alpha_{n}=\frac{\left\{\frac{1}{t}\left(2 a_{0} \partial_{t}+3 a_{1} \partial_{a_{0}}+4 a_{2} \partial_{a_{1}}+\ldots\right)\right\}^{n}\left(-\frac{a_{0}}{t^{3}}\right)}{3.4 .5 \ldots(n+2)}
\end{aligned}
$$

A proof of the formula

$$
a_{n}=-t^{-n-3}\left(e^{-\frac{V}{t}}\right) a_{n},
$$

obtained by Mr Hammond, in which

$$
V=4 \cdot \frac{a_{0}^{2}}{2} \partial_{a_{1}}+5 a_{0} a_{1} \partial_{a_{2}}+6\left(a_{0} a_{2}+\frac{a_{1}^{2}}{2}\right) \partial_{a_{3}}+7\left(a_{0} a_{3}+a_{1} a_{2}\right) \partial_{a_{4}}+\ldots,
$$

will be given later on, when we treat of this operator, which, in the theory of Reciprocants, is the analogue of the operator $a \partial_{b}+2 b \partial_{c}+3 c \partial_{d}+\ldots$, with which we are familiarly acquainted in the theory of Invariants. 
Consider the expression

$$
c t-5 a b \text {. }
$$

If, in $\gamma \tau-5 \alpha \beta$, which may be called its transform, we write

$$
\tau=\frac{1}{t}, \quad \alpha=-\frac{a}{t^{3}}, \quad \beta=\frac{-b t+3 a^{2}}{t^{5}}, \quad \gamma=\frac{-c t^{2}+10 a b t-15 a^{3}}{t^{7}},
$$

this becomes a fraction whose denominator is $t^{8}$, while its numerator is

$$
-c t^{2}+10 a b t-15 a^{3}+5 a\left(-b t+3 a^{2}\right)=-c t^{2}+5 a b t .
$$

Removing the common factor $t$ from the numerator and denominator of this fraction, we have

$$
\gamma \tau-5 a \beta=-\frac{c t-5 a b}{t^{7}}
$$

Here, then, as in the case of the well-known monomial for which

$$
a=-t^{3} \alpha,
$$

and the Schwarzian for which

the expression

$$
\begin{aligned}
2 b t-3 a^{2} & =-t^{6}\left(2 \beta \tau-3 \alpha^{2}\right), \\
c t-5 a b & =-t^{7}(\gamma \tau-5 \alpha \beta)
\end{aligned}
$$

changes its sign on reciprocation.

That reciprocation is not always accompanied with a change of sign will be clear if we consider the product of any pair of the three expressions given above. Or we may take, as an example of a reciprocant in which this change of sign does not occur, the form

$$
3 a c-5 b^{2} .
$$

Here $\quad 3 a \gamma-5 \beta^{2}=\frac{3 a\left(c t^{2}-10 a b t+15 a^{3}\right)-5\left(b t-3 a^{2}\right)^{2}}{t^{10}}$.

In the fraction on the right-hand side the only surviving terms of the numerator are those containing the highest power of $t$, the rest destroying one another. Thus

$$
3 a \gamma-5 \beta^{2}=\frac{1}{t^{8}}\left(3 a c-5 b^{2}\right) .
$$

Reciprocants which change their sign when the variables $x$ and $y$ are interchanged, will be said to be of odd character; those, on the contrary, which keep their sign unchanged will be said to be of even character. The distinction is an important one, and will be observed in what follows.

Forms such as the one just considered, where $t$ does not appear in the form itself, but only in the extraneous factor, will be called Pure Reciprocants, in order to distinguish them from those forms (of which the Schwarzian $2 t b-3 a^{2}$ is an example) into which $t$ enters, which will be called Mixed Reciprocants. It will be seen hereafter that Pure Reciprocants are the analogues of the invariants of Binary Quantics. 
With modified letters (that is, writing $a=2 a_{0}, b=6 a_{1}$, and $c=24 a_{2}$ )

$$
3 a c-5 b^{2} \text { becomes } 144 a_{0} a_{2}-180 a_{1}^{2}=36\left(4 a_{0} a_{2}-5 a_{1}^{2}\right) \text {. }
$$

Operating on this with

$$
V=2 a^{2} \partial_{a_{1}}+5 a_{0} a_{1} \partial_{a_{2}}+\ldots
$$

we have

$$
V\left(4 a_{0} a_{2}-5 a_{1}^{2}\right)=0
$$

We shall prove subsequently that all Pure Reciprocants are, in like manner, subject to annihilation by the operator $V$.

Hitherto we have only considered homogeneous forms; let us now take as an example of a non-homogeneous reciprocant the expression

$$
\left(1+t^{2}\right) b-3 a^{2} t
$$

Here

$$
\begin{aligned}
\left(1+\tau^{2}\right) \beta-3 \alpha^{2} \tau & =\left(1+\frac{1}{t^{2}}\right)\left(\frac{-b t+3 a^{2}}{t^{5}}\right)-\frac{3 a^{2}}{t^{7}} \\
& =\frac{\left(1+t^{2}\right)\left(-b t+3 a^{2}\right)-3 a^{2}}{t^{7}}
\end{aligned}
$$

In the numerator of this fraction the terms $+3 a^{2}$ and $-3 a^{2}$ cancel, a factor $t$ divides out, and we have finally

$$
\left(1+\tau^{2}\right) \beta-3 \alpha^{2} \tau=-\frac{\left(1+t^{2}\right) b-3 a^{2} t}{t^{6}} .
$$

In general, a Reciprocant may be defined to be a function $F$ of such a kind that $F(\tau, \alpha, \beta, \gamma, \ldots)$ contains $F(t, a, b, c, \ldots)$ as a factor. An important special case is that in which the other factor is merely numerical; the function $F$ is then said to be an Absolute Reciprocant.

When we limit ourselves to the case where $F$ is a rational integral function of the letters, it may be proved that

$$
F(t, a, b, c, \ldots)= \pm t^{\mu} F(\tau, \alpha, \beta, \gamma, \ldots)
$$

For, in the first place, since any one of the letters $\alpha, \beta, \gamma, \ldots$ is a rational function of $t, a, b, c, \ldots$ and integral with respect to all of them except $t$, containing only a power of this letter in the denominator, it is clear that any rational integral function of $\tau, \alpha, \beta, \gamma, \ldots$ such as $F(\tau, \alpha, \beta, \gamma, \ldots)$ is supposed to be, must be a rational integral function of $t, a, b, c, \ldots$ divided by some power of $t$. But since $F$ is a reciprocant, $F(\tau, \alpha, \beta, \gamma, \ldots)$ must contain $F(t, a, b, c, \ldots)$ as a factor; and if we suppose the other factor to be

$$
\frac{\phi(t, a, b, c, \ldots)}{t^{\lambda}}
$$

we must have

$$
F(\tau, \alpha, \beta, \gamma, \ldots)=\frac{\phi(t, a, b, c, \ldots)}{t^{\lambda}} F(t, a, b, c, \ldots),
$$

where $\phi$ is rational and integral with respect to all the letters. 
Moreover, $F(t, a, b, c, \ldots)=\frac{\phi(\tau, \alpha, \beta, \gamma, \ldots)}{\tau^{\lambda}} F(\tau, \alpha, \beta, \gamma, \ldots)$.

Hence we must have identically

$$
\phi(t, a, b, c, \ldots) \phi(\tau, \alpha, \beta, \gamma, \ldots)=1,
$$

where, on the supposition that the functions $\phi$ contain other letters besides $t$ and $\tau, \phi(t, a, b, c, \ldots)$ is, and $\phi(\tau, \alpha, \beta, \gamma, \ldots)$ can be expressed as, a rational function integral as regards the letters $a, b, c, \ldots$. But this supposition is manifestly inadmissible, for the product of two integral rational functions of $a, b, c, \ldots$ cannot be identically equal to unity. Hence $t$ is the only letter that can appear in the extraneous factor and we may write

$$
F(\tau, \alpha, \beta, \gamma, \ldots)=\frac{\psi(t)}{t^{\lambda}} F(t, a, b, c, \ldots)
$$

where $\psi(t)$ is a rational integral function.

The same reasoning as before shows that we must have identically

$$
\psi(t) \psi(\tau)=1
$$

But this cannot be true if $\psi(t)$ has any root different from zero; for if we give $t$ such a value as will make $\psi(t)$ vanish, this value must also make $\psi(\tau)$ infinite; and since

$$
\begin{aligned}
\psi(\tau) & =A+B \tau+C \tau^{2}+\ldots+M \tau^{m} \\
& =A+\frac{B}{t}+\frac{C}{t^{2}}+\ldots+\frac{M}{t^{m}}
\end{aligned}
$$

the only value of $t$ for which $\psi(\tau)$ becomes infinite is a zero value. Hence $\psi(t)$ is of the form $M t^{m}$, and consequently $\psi(\tau)=M \tau^{m}$. Thus

and therefore

$$
\psi(t) \psi(\tau)=M^{2} t^{m} \tau^{m}=1
$$

$$
M^{2}=1 \text {. }
$$

We have now proved that if $F$ is a rational integral reciprocant,

or we may say,

$$
\begin{aligned}
F(t, a, b, c, \ldots) & = \pm t^{\mu} F(\tau, \alpha, \beta, \gamma, \ldots) \\
& =(-)^{\kappa} t^{\mu} F(\tau, \alpha, \beta, \gamma, \ldots)
\end{aligned}
$$

where $\kappa=1$ or 0 according as the reciprocant is of odd or even character.

It obviously follows that the product or quotient of any two rational integral reciprocants is itself a reciprocant; but it must be carefully observed that this is not true of their sum or difference unless certain conditions are fulfilled. For if we write

and

$$
F_{1}(t, a, \ldots)=(-)^{\kappa_{1}} t^{\mu_{1}} F_{1}(\tau, \alpha, \ldots)
$$

we see that

$$
F_{2}(t, a, \ldots)=(-)^{\kappa_{2}} t^{\mu_{2}} F_{2}(\tau, \alpha, \ldots)
$$

$$
p F_{1}(t, a, \ldots)+q F_{2}(t, a, \ldots)=(-)^{\kappa_{1}} t^{\mu_{1}} p F_{1}(\tau, \alpha, \ldots)+(-)^{\kappa_{2}} t^{\mu_{2}} q F_{2}(\tau, \alpha, \ldots),
$$


and consequently this expression will be a reciprocant if $\kappa_{1}=\kappa_{2}$ and $\mu_{1}=\mu_{2}$, but not otherwise. If we call the index of $t$ in the extraneous factor the characteristic, what we have proved is that no linear function of two reciprocants can be a reciprocant, unless they have the same characteristic and are of the same character. In dealing with Absolute Reciprocants, since the characteristic of these is always zero, we need only attend to their character.

I propose for the present to confine myself to homogeneous and isobaric reciprocants*, that is, to such as are homogeneous and isobaric when the letters $t, a, b, c, \ldots$ are considered to be each of degree 1 , their respective weights being $-1,0,1,2, \ldots$. The letter $w$ will be used to denote the weight of such a reciprocant, $i$ its degree, and $j$ its extent, that is, the weight of the most advanced letter which it contains.

Let any such reciprocant $F^{\prime}(t, a, b, c, \ldots)$ contain a term $A t^{v} a^{l} b^{m} c^{n} \ldots$, then

and

$$
\begin{aligned}
& v+l+m+n+\ldots=i \\
& -v+m+2 n+\ldots=w .
\end{aligned}
$$

The corresponding term in $F(\tau, \alpha, \beta, \gamma, \ldots)$ will be $A \tau^{v} \alpha^{l} \beta^{m} \gamma^{n} \ldots$ where

$$
\tau=\frac{1}{t}, \alpha=-\frac{a}{t^{3}}, \quad \beta=-\frac{b}{t^{4}}+\ldots, \quad \gamma=-\frac{c}{t^{5}}+\ldots, \text { etc. }
$$

Now, if no term of $F$ contains a smaller number of the letters $a, b, c, \ldots$ than are found in the term we are considering, the first terms of $\beta, \gamma$, etc., may be taken instead of these quantities themselves and $A \tau^{v} \alpha^{l} \beta^{m} \gamma^{n} \ldots$ may be replaced by

$$
(-)^{l+m+n+\ldots} A t^{-v-3 l-4 m-5 n-\ldots} a^{l} b^{m} c^{n} \ldots=(-)^{i-v} A t^{v-3 i-w} a^{l} b^{m} c^{n} \ldots
$$

But since $\quad F(t, a, b, c, \ldots)=(-)^{\kappa} t^{\mu} F(\tau, \alpha, \beta, \gamma, \ldots)$

we must have identically

$$
A t^{v} a^{l} b^{m} c^{n} \ldots=(-)^{i-v+\kappa} A t^{\mu+v-3 i-w} a^{l} b^{m} c^{n} \ldots
$$

Hence the character is even or odd according to the parity of $i-v$ (that is, of the smallest number of letters different from $t$ in any term), and the characteristic $\mu=3 i+w$.

The type of a reciprocant depends on the character, weight, degree and extent. As the extraneous factor is always of the form $(-)^{\kappa} t^{\mu}$, where $\kappa$ is 1 or 0 , we may define the type of a reciprocant by

$$
1: w: i, j \text { or } 0: w: i, j
$$

according as its character is odd or even.

For Pure Reciprocants the smallest number of letters different from $t$ in any term is (since all the letters are different from $t$ ) the same as its degree.

\footnotetext{
* Here and elsewhere the word reciprocant is used in the sense of rational integral reciprocant: this will always be done when there is no danger of confusion arising from it.
} 
Hence the character of a Pure Reciprocant is odd or even according to the parity of $i$, and for this reason the type of a Pure Reciprocant may be defined by $w: i, j$.

A linear combination of reciprocants of the same type will be a reciprocant, for when the type is known both the character and characteristic are given.

\section{LECTURE III.}

Let $F$ be any function (not necessarily homogeneous or even algebraical) of the differential derivatives which acquires a numerical multiplier $M$, but is otherwise unchanged when the reciprocal substitution of $x$ for $y$ and $y$ for $x$ is effected. A second reciprocation multiplies the function again by $M$, and thus the total effect of both substitutions is to multiply $F$ by $M^{2}$. But since the second reciprocation reproduces the original function, we must have $M^{2}=1$. Functions of this kind are therefore unaltered by reciprocation (except it may be in sign), and for this reason are called Absolute Reciprocants. These, as we shall presently see, play an important part in the general theory. Like all other reciprocants, they range naturally in two distinct classes, those of odd and those of even character.

It is perhaps worthy of notice that the extraneous factor of a general reciprocant is the exponential of an absolute reciprocant of odd character. For if

$$
F(t, a, b, c, \ldots)=\phi(t, a, b, c, \ldots) F(\tau, \alpha, \beta, \gamma, \ldots),
$$

we must still have, as before,

$$
\phi(t, a, b, c, \ldots) \phi(\tau, \alpha, \beta, \gamma, \ldots)=1
$$

that is $\quad \log \phi(t, a, b, c, \ldots)=-\log \phi(\tau, \alpha, \beta, \gamma, \ldots)$;

or $\log \phi(t, a, b, c, \ldots)$ is an absolute reciprocant of odd character.

An absolute reciprocant may be obtained from any pair of rational integral reciprocants in the same way that an absolute invariant is found from two ordinary invariants. For let

and

$$
\begin{aligned}
& F_{1}(t, a, b, c, \ldots)=(-)^{\kappa_{1}} t^{\mu_{1}} F_{1}(\tau, \alpha, \beta, \gamma, \ldots), \\
& F_{2}(t, a, b, c, \ldots)=(-)^{\kappa_{2}} t^{\mu_{2}} F_{2}(\tau, \alpha, \beta, \gamma, \ldots),
\end{aligned}
$$

then $\quad \frac{\left\{F_{1}(t, a, b, c, \ldots)\right\}^{\mu_{2}}}{\left\{F_{2}(t, a, b, c, \ldots)\right\}^{\mu_{1}}}=(-)^{\kappa_{1} \mu_{2}-\kappa_{2} \mu_{1}} \frac{\left\{F_{1}(\tau, \alpha, \beta, \gamma, \ldots)\right\}^{\mu_{2}}}{\left\{F_{2}(\tau, \alpha, \beta, \gamma, \ldots)\right\}^{\mu_{1}}}$

or we may say that $F_{1}^{\mu_{2}} \div F_{2}^{\mu_{1}}$ is an absolute reciprocant of even or odd character according to the parity of $\kappa_{1} \mu_{22}-\kappa_{2} \mu_{1}$. 
Thus, for example, from

$$
\begin{aligned}
a & =-t^{3} \alpha \\
\text { and } & 3 a c-5 b^{2}=t^{8}\left(3 \alpha \gamma-5 \beta^{2}\right)
\end{aligned}
$$

we form $\frac{\left(3 a c-5 b^{2}\right)^{3}}{a^{8}}$, an absolute reciprocant of even character.

From a reciprocant $F$ whose characteristic is $\mu$ we obtain an absolute reciprocant of the same character as $F$ by dividing it by $t^{\frac{\mu}{2}}$.

For if we only remember that $\tau=\frac{1}{t}$, it obviously follows that

$$
F(t, a, b, c, \ldots)= \pm t^{\mu} F(\tau, \alpha, \beta, \gamma, \ldots)
$$

can be written in the form

$$
\frac{F(t, a, b, c, \ldots)}{t^{\frac{\mu}{2}}}= \pm \frac{F(\tau, \alpha, \beta, \gamma, \ldots)}{\tau^{\frac{\mu}{2}}},
$$

where the original character of the reciprocant $F$ is preserved.

It may be noticed that a reciprocant of odd character cannot be divided by $\sqrt{ }(-1) t^{\frac{\mu}{2}}$ so as to give an absolute reciprocant of even character; for, the reciprocal of $F$ being $-t^{\mu} F^{\prime}$, that of $F \div \sqrt{ }(-1) t^{\frac{\mu}{2}}$ will still be $-F^{\prime} \div \sqrt{ }(-1) \tau^{\frac{\mu}{2}}$. The character of a reciprocant is thus seen to be one of its indelible attributes.

As simple examples of absolute reciprocants we may take $\frac{3 a c-5 b^{2}}{t^{4}}$, which becomes on reciprocation $\frac{3 a \gamma-5 \beta^{2}}{\tau^{4}}$, and $\frac{a}{t^{\frac{3}{2}}}$, which reciprocates into $-\frac{a}{\tau^{\frac{3}{2}}}$. The character of the former is even, that of the latter odd.

Observing that

$$
\log t=-\log \tau \text { and } \frac{1}{\sqrt{ } t} \cdot \frac{d}{d x}=\frac{1}{\sqrt{ } \tau} \cdot \frac{d}{d y}
$$

we have

$$
\left(\frac{1}{\sqrt{ } t} \cdot \frac{d}{d x}\right) \log t=-\left(\frac{1}{\sqrt{ } \tau} \cdot \frac{d}{d y}\right) \log \tau
$$

From this, in like manner, we obtain

and so, in general,

$$
\left(\frac{1}{\sqrt{ } t} \cdot \frac{d}{d x}\right)^{2} \log t=-\left(\frac{1}{\sqrt{ } \tau} \cdot \frac{d}{d y}\right)^{2} \log \tau
$$

$$
\left(\frac{1}{\sqrt{ } t} \cdot \frac{d}{d x}\right)^{i} \log t=-\left(\frac{1}{\sqrt{ } \tau} \cdot \frac{d}{d y}\right)^{i} \log \tau
$$


Hence $\left(\frac{1}{\sqrt{ } t} \cdot \frac{d}{d x}\right)^{i} \log t$ is an absolute reciprocant, and of an odd character, for all positive integral values of $i$. We thus obtain a series of fractions with rational integral homogeneous reciprocants in their numerators and powers of $t^{\frac{3}{2}}$ in their denominators. It will be sufficient, before proceeding to the more general theory of Eduction, as it may be called, to examine, by way of illustration, the cases in which $i=1,2$ and 3 .

Let $i=1$; then

$$
\left(\frac{1}{\sqrt{ } t} \cdot \frac{d}{d x}\right) \log t=\frac{a}{t^{\frac{3}{2}}}
$$

So that, in the case where $i=2$, we have

$$
\begin{aligned}
\left(\frac{1}{\sqrt{ } t} \cdot \frac{d}{d x}\right)^{2} \log t & =\left(\frac{1}{\sqrt{ } t} \cdot \frac{d}{d x}\right) \frac{a}{t^{\frac{3}{2}}}=\frac{b}{t^{2}}-\frac{3}{2} \cdot \frac{a^{2}}{t^{3}} \\
& =\frac{2 b t-3 a^{2}}{2 t^{3}}
\end{aligned}
$$

The numerator of this fraction is the Schwarzian.

In like manner, when $i=3$,

$$
\left(\frac{1}{\sqrt{ } t} \cdot \frac{d}{d x}\right)^{3} \log t=\left(\frac{1}{\sqrt{ } t} \cdot \frac{d}{d x}\right)\left(\frac{2 b t-3 a^{2}}{2 t^{3}}\right)=\frac{2 c t-4 a b}{2 t^{\frac{7}{2}}}-\frac{6 a b t-9 a^{3}}{t^{\frac{9}{2}}}=\frac{2 c t^{2}-10 a b t+9 a^{3}}{2 t^{\frac{9}{2}}}
$$

But here a reduction may be effected, for $\left(\frac{a}{t^{\frac{3}{2}}}\right)^{3}$, as well as $\frac{a}{t^{\frac{3}{2}}}$ itself, is an absolute reciprocant of the same character as the whole of the expression just found. Hence we may reject the term $\frac{9}{2} \cdot \frac{a^{3}}{t^{\frac{9}{2}}}$ without therehy affecting the reciprocantive property of the form, and thus obtain

$$
\frac{c t-5 a b}{t^{\frac{7}{2}}}
$$

an absolute reciprocant of odd character. The corresponding rational integral reciprocant is

$$
c t-5 a b \text {. }
$$

We have found that $\frac{a}{t^{\frac{3}{2}}}$ and $\frac{2 b t-3 a^{2}}{t^{3}}$ are each of them reciprocants. Why, then, by parity of reasoning, is not $\frac{2 b t}{t^{3}}$, and therefore $b$, a reciprocant? It is because $\frac{a^{2}}{t^{3}}$, the square of $\frac{a}{t^{\frac{3}{2}}}$, is of even character, while $\frac{2 b t-3 a^{2}}{t^{3}}$ is of an odd character, so that no linear combination of the two would be legitimate. 
If we differentiate any absolute reciprocant with respect to $x$, we shall obtain another reciprocant of the same character. For let $R$ be any absolute reciprocant and $R^{\prime}$ its transform, then

$$
R= \pm R^{\prime}
$$

and since $\frac{d}{d x}=t \frac{d}{d y}$ may be written in the equivalent but more symmetrical form

$$
\frac{1}{\sqrt{ } t} \cdot \frac{d}{d x}=\frac{1}{\sqrt{ } \tau} \cdot \frac{d}{d y},
$$

we have

$$
\left(\frac{1}{\sqrt{ } t} \cdot \frac{d}{d x}\right) R= \pm\left(\frac{1}{\sqrt{ } \tau} \cdot \frac{d}{d y}\right) R^{\prime}
$$

On one side of this identical equation is a function of the differential derivatives of $y$ with respect to $x$; on the other, a precisely similar function of those of $x$ with respect to $y$. Hence $\frac{1}{\sqrt{ } t} \cdot \frac{d R}{d x}$ is an absolute reciprocant, and therefore $\frac{d R}{d x}$ is a reciprocant, the character of each being the same as that of $R$.

I will avail myself of the conclusion just obtained, which is the cardinal property of absolute reciprocants, to give a general method of generating from any given Rational Integral Reciprocant an infinity of others-rational integral educts of it, we may say. Let $F$ be such a reciprocant, and $\mu$ its characteristic; then $\frac{F}{t^{\frac{\mu}{2}}}$ is an absolute reciprocant, and consequently $\frac{d}{d x}\left(\frac{F}{\frac{\mu}{\frac{\mu}{2}}}\right)$ is a reciprocant, both of them of the same character as $F$; that is

$$
\frac{t \frac{d F}{d x}-\frac{\mu}{2} \cdot a F}{t^{\frac{\mu}{2}+1}}
$$

or we may say

$$
2 t \frac{d F}{d x}-\mu a F
$$

is a reciprocant of the same character as $F$.

This is even true for non-homogeneous reciprocants, for the only assumption made at present as to the nature of $F$ is that it is a rational integral reciprocant. But if we further assume that it is homogeneous and isobaric*, we know that

$$
\mu=3 i+w .
$$

Now, Euler's equation gives

$$
3 i=3\left(t \partial_{t}+a \partial_{a}+b \partial_{b}+c \partial_{c}+\ldots\right)
$$

* It will subsequently be proved that every rational integral reciprocant which is homogeneous is also isobaric. 
and from the similar equation for isobaric functions (remembering that the weights of $t, a, b, c, \ldots$ are $-1,0,1,2, \ldots)$ we obtain

$$
w=-t \partial_{t}+b \partial_{b}+2 c \partial_{c} \ldots
$$

so that

$$
\mu=2 t \partial_{t}+3 a \partial_{a}+4 b \partial_{b}+5 c \partial_{c}+\ldots .
$$

And since

$$
\frac{d}{d x}=a \partial_{t}+b \partial_{a}+c \partial_{b}+d \partial_{c}+\ldots
$$

we may in $\left(2 t \frac{d}{d x}-\mu a\right) F$ replace $2 t \frac{d}{d x}-\mu a$ by

$$
\begin{gathered}
2 t\left(a \partial_{t}+b \partial_{a}+c \partial_{b}+d \partial_{c}+\ldots\right) \\
-a\left(2 t \partial_{t}+3 a \partial_{a}+4 b \partial_{b}+5 c \partial_{c}+\ldots\right),
\end{gathered}
$$

or by its equivalent

$$
\left(2 b t-3 a^{2}\right) \partial_{a}+(2 c t-4 a b) \partial_{b}+(2 d t-5 a c) \partial_{c}+\ldots .
$$

The conclusion arrived at is that when $F$ is a rational integral homogeneous reciprocant,

$$
\left\{\left(2 b t-3 a^{2}\right) \partial_{a}+(2 c t-4 a b) \partial_{b}+(2 d t-5 a c) \partial_{c}+\ldots\right\} F
$$

is another, and that both are of the same character.

It will be convenient to use the letter $G$ to denote the operator just found and to speak of it as the generator for mixed reciprocants. By the repeated operation of this generator on a we may obtain the series $G a, G^{2} a, G^{3} a, \ldots$, whose terms will be mixed reciprocants, since each operation increases the highest power of $t$ by unity. The forms thus obtained will, in general, not be irreducible. It is, in fact, easy to see that a reduction must always take place at every second step. Observing that $G F$ only expresses the numerator of the absolute reciprocant $\frac{1}{\sqrt{ } t} \cdot \frac{d}{d x}\left(\frac{F}{t^{\frac{\mu}{2}}}\right)$ in a convenient form, and that $G^{2} F$ is equivalent to the numerator of $\left(\frac{1}{\sqrt{ } t} \cdot \frac{d}{d x}\right)^{2}\left(\frac{F}{t^{2}}\right)$, we have

$$
\frac{1}{\sqrt{ } t} \cdot \frac{d}{d x}\left(\frac{F}{t^{\frac{\mu}{2}}}\right)=\frac{t \frac{d F}{d x}-\frac{\mu}{2} \cdot a F}{t^{\frac{\mu+3}{2}}}
$$

so that $\left(\frac{1}{\sqrt{ } t} \cdot \frac{d}{d x}\right)^{2}\left(\frac{F}{\frac{\mu}{2}}\right)=\frac{1}{\sqrt{ } t} \cdot \frac{d}{d x}\left(\frac{t \frac{d F}{d x}-\frac{\mu}{2} \cdot a F}{t^{\frac{\mu+3}{2}}}\right)$

$$
=\frac{t \frac{d}{d x}\left(t \frac{d F}{d x}-\frac{\mu}{2} \cdot a F\right)-\frac{\mu+3}{2} \cdot a\left(t \frac{d F}{d x}-\frac{\mu}{2} \cdot a F\right)}{t^{\frac{\mu}{2}+3}} .
$$


The whole of this fraction is an absolute reciprocant of the same character as $F$; so also is $\frac{a^{2} F}{t^{\frac{\mu}{2}+3}}$ (the product of the even absolute reciprocant $\frac{a^{2}}{t^{3}}$ by $\left.\frac{F}{\frac{\mu}{2}}\right)$. We may therefore reject the term $\frac{\mu}{2} \cdot \frac{\mu+3}{2} \cdot a^{2} F$ from the numerator, and the remaining fraction

$$
\frac{\frac{d}{d x}\left(t \frac{d F}{d x}-\frac{\mu}{2} \cdot a F\right)-\frac{\mu+3}{2} \cdot a \frac{d F}{d x}}{t^{\frac{\mu}{2}+2}}
$$

will still be an absolute reciprocant of the same character as $F$. Its numerator, which is one degree lower than $G^{2} F$, may be written in the form

$$
t \frac{d^{2} F}{d x^{2}}-\left(\mu+\frac{1}{2}\right) a \frac{d F}{d x}-\frac{\mu}{2} b F
$$

This, it may be noticed, is a reciprocant of the same character as $F$, even when $F$ is non-homogeneous.

Starting with $a$, we have

$$
\begin{aligned}
G a & =2 b t-3 a^{2}(\text { the Schwarzian), } \\
G^{2} a & =G\left(2 b t-3 a^{2}\right)=-6 a\left(2 b t-3 a^{2}\right)+2 t(2 c t-4 a b)=4 c t^{2}-20 a b t+18 a^{3} .
\end{aligned}
$$

But, for the reason previously given, $18 a^{3}$ may be removed, so that rejecting this term and dividing out by $4 t$ we obtain the form

$$
c t-5 a b \text {, }
$$

which may be called the Post-Schwarzian.

The next form is obtained by operating on the Post-Schwarzian with $G$; thus, we have to calculate the value of $G(c t-5 a b)$, where

$$
G=\left(2 b t-3 a^{2}\right) \partial_{a}+(2 c t-4 a b) \partial_{b}+(2 d t-5 a c) \partial_{c} .
$$

The working may be arranged as follows:

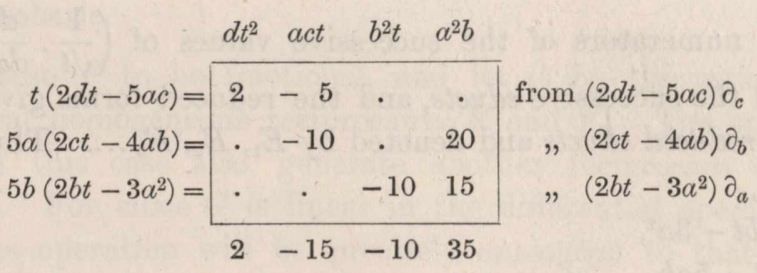

The result should be read thus :

$$
2 d t^{2}-15 a c t-10 b^{2} t+35 a^{2} b .
$$

To obtain the next of this series of reciprocants, we have to operate on this with $G$ and at the same time to take account of the reduction that has 
to be made at each alternate step. The arrangement of the work is similar to that of the former case.

$$
\begin{aligned}
& e t^{3} \quad a d t^{2} \quad b c t^{2} \quad a^{2} c t \quad a b^{2} t \quad a^{3} b \\
& 2 t^{2}(2 e t-6 a d)=\sqrt{4-12 \quad . \quad . \quad . \quad . \quad \text { from }(2 e t-6 a d) \partial_{d}} \\
& -15 a t(2 d t-5 a c)=.-30 \quad \cdot \quad 75 . \quad . \quad, \quad(2 d t-5 a c) \partial_{c} \\
& \left(35 a^{2}-20 b t\right)(2 c t-4 a b)=\begin{array}{lllllll}
. & -40 & 70 & 80 & -140 \quad, \quad(2 c t-4 a b) \partial_{b}
\end{array}
\end{aligned}
$$

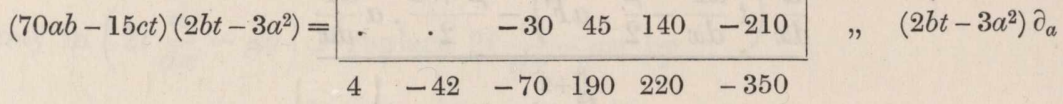

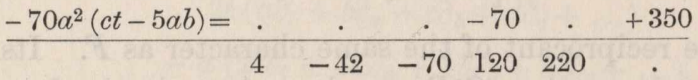

This divides by $2 t$, giving the reduced value

$$
2 e t^{2}-21 a d t-35 b c t+60 a^{2} c+110 a b^{2} .
$$

The next obtained by this process will be seen by the following work to be

$4 f t^{3}-56 a e t^{2}-112 b d t^{2}-70 c^{2} t^{2}+309 a^{2} d t+995 a b c t+220 b^{3} t-660 a^{3} c-1210 a^{2} b^{2}$.

$$
\begin{aligned}
& f t^{3} a e t^{2} \quad b d t^{2} \quad c^{2} t^{2} \quad a^{2} d t a b c t \quad b^{3} t \quad a^{3} c \quad a^{2} b^{2} \\
& 2 t^{2}(2 f t-7 a e)=4-14 \text {. . . . . . . from }(2 f t-7 a e) \partial_{e} \\
& -21 a t(2 e t-6 a d)=-42 \quad . \quad .126 \quad . \quad . \quad . \quad \%(2 e t-6 a d) \partial_{d} \\
& \left(-35 b t+60 a^{2}\right)(2 d t-5 a c)=. \quad . \quad-70 \quad . \quad 120175 \quad . \quad-300 \quad . \quad \%(2 d t-5 a c) \partial_{c} \\
& (-35 c t+220 a b)(2 c t-4 a b)=. \quad . \quad . \quad-70 \quad .580 \text {. } \quad . \quad-880 \quad \#(2 c t-4 a b) \partial_{b}
\end{aligned}
$$

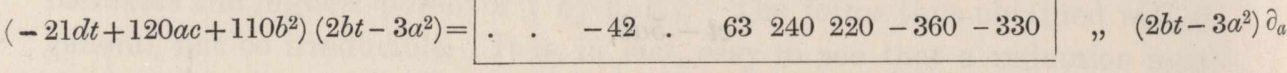

$$
\begin{aligned}
& 4-56-112-70309995220-660-1210
\end{aligned}
$$

This cannot be reduced in the same manner as the preceding form, but it must not be supposed that the forms thus obtained are in general irreducible.

Having regard to the circumstance that the forms of the series

$$
a, G a, G^{2} a, \ldots
$$

occur in the numerators of the successive values of $\left(\frac{1}{\sqrt{ } t} \cdot \frac{d}{d x}\right)^{n} \log t$, they may be called the successive educts, and the reduced forms given above may be called the reduced educts and denoted by $E_{1}, E_{2}, E_{3} \ldots$ Thus

$$
\begin{aligned}
& E_{1}=a \\
& E_{2}=2 b t-3 a^{2}, \\
& E_{3}=c t-5 a b, \\
& E_{4}=2 d t^{2}-15 a c t-10 b^{2} t+35 a^{2} b, \\
& E_{5}=2 e t^{2}-21 a d t-35 b c t+60 a^{2} c+110 a b^{2}, \\
& E_{6}=4 f t^{3}-56 a e t^{2}-112 b d t^{2}-70 c^{2} t^{2}+309 a^{2} d t+995 a b c t \\
& +220 b^{3} t-660 a^{3} c-1210 a^{2} b^{2} .
\end{aligned}
$$




\section{LECTURE IV.}

We have seen that when $F$ is a rational integral homogeneous and isobaric reciprocant, $G F$ is another of the same character. It will now appear that the condition of isobarism is implied in that of homogeneity; for let $F$ be a rational integral homogeneous reciprocant, $\mu$ its characteristic and $i$ its degree in the letters $t, a, b, c, \ldots$, then, in the identical equation

$$
F(t, a, b, c, \ldots)= \pm t^{\mu} F^{\prime}(\tau, \alpha, \beta, \gamma, \ldots)
$$

both members are homogeneous and of the same degree in the letters $t, a, b, c, \ldots$; that is, if $A t^{k} a^{l} b^{m} c^{n} \ldots$ be any term of $F(t, a, b, c, \ldots)$, its degree must be the same as that of $t^{\mu} A \tau^{k} \alpha^{l} \beta^{m} \gamma^{n} \ldots$ when $\tau, \alpha, \beta, \gamma, \ldots$ are expressed in terms of $t, a, b, c, \ldots$ But

$$
\tau=\frac{1}{t}, \quad \alpha=-\frac{a}{t^{3}}, \quad \beta=-\frac{b}{t^{4}}+\ldots, \quad \gamma=-\frac{c}{t^{5}}+\ldots
$$

and so on. The degrees of $\tau, \alpha, \beta, \gamma, \ldots$ are therefore $-1,-2,-3,-4, \ldots$ respectively. Hence

$$
k+l+m+n+\ldots=\mu-k-2 l-3 m-4 n-\ldots,
$$

or

And by hypothesis

$$
\mu=2 k+3 l+4 m+5 n+\ldots .
$$

so that

$$
i=k+l+m+n+\ldots \text {, }
$$

$$
\mu-3 i=-k+m+2 n+\ldots .
$$

Neither $\mu$ nor $i$ is dependent for its value on the selection of a particular term of $F$, for all terms of $F(\tau, \alpha, \beta, \gamma, \ldots)$ are multiplied by the same extraneous factor $\pm t^{\mu}$, and all terms of $F(t, a, b, c, \ldots)$ are of the same degree $i$. Hence $-k+m+2 n+\ldots$ must also be the same for each term of $F$; or, attributing the weights $-1,0,1,2, \ldots$ to the letters $t, a, b, c, \ldots$, the function $F$ is isobaric.

Next, suppose $F$ to be fractional, and let it be the ratio of the two rational integral homogeneous reciprocants $F_{1}$ and $F_{2}$. The operation of $G$ on $F$ will, in this case also, generate another reciprocant of the same character as $F$. For, since $G$ is linear in the differential operative symbols $\partial_{a}, \partial_{b}, \partial_{c}, \ldots$, its operation will be precisely analogous to that of differentiation, so that, operating with $G$ on

$$
\begin{gathered}
F=\frac{F_{1}}{F_{2}}, \\
\text { we have } \quad G F=\frac{F_{2} G F_{1}-F_{1} G F_{2}}{F_{2}^{2}} .
\end{gathered}
$$


In order to prove that this is a reciprocant, we have to show that the character and characteristic are the same for both terms of the numerator. But $G F_{1}$ is a reciprocant of the same character as $F_{1}$, and $G F_{2}$ is one of the same character as $F_{2}$; thus the two terms of the numerator are of the same character as $F_{1} F_{2}$. As regards the characteristic, it should be noticed that $G$, that is, the operator $\left(2 b t-3 a^{2}\right) \partial_{a}+(2 c t-4 a b) \partial_{b}+\ldots$, increases the degree by unity, but does not alter the weight, so that it increases the characteristic of any rational integral homogeneous reciprocant by 3 . Thus the characteristic of each term in the numerator exceeds by 3 that of $F_{1} F_{2}$. Hence $G F$ is a reciprocant, and, taking account of its denominator as well as its numerator, we see that the operation of $G$ on a rational homogeneous reciprocant, whether fractional or integral, produces another in which the original character is preserved while the characteristic is increased by three units.

More generally, let $F_{1}, F_{2}, F_{3}, \ldots$ be any rational homogeneous reciprocants whose extraneous factors are $(-)^{\kappa_{1}} t^{\mu_{1}},(-)^{\kappa_{2}} t^{\mu_{2}},(-)^{\kappa_{3}} t^{\mu_{3}}, \ldots$ respectively ; and suppose $\Phi$ to consist of a series of terms of the form $A F_{1}^{\lambda_{1}} F_{2}^{\lambda_{2}} F_{3}^{\lambda_{3}} \ldots$, such that the extraneous factor for each term is $(-)^{\kappa} t^{\mu}$. Then $\Phi$ is a reciprocant, but not necessarily a rational one; for the indices $\lambda_{1}, \lambda_{2}, \lambda_{3}, \ldots$ may be supposed fractional, provided only that they satisfy the conditions

$$
\kappa_{1} \lambda_{1}+\kappa_{2} \lambda_{2}+\kappa_{3} \lambda_{3}+\ldots-\kappa=\text { a positive or negative even integer, }
$$

and

$$
\mu_{1} \lambda_{1}+\mu_{2} \lambda_{2}+\mu_{3} \lambda_{3}+\ldots-\mu=0 .
$$

We proceed to show that $G \Phi$ is also a reciprocant, and that its extraneous factor is $(-)^{\kappa} t^{\mu+3}$. Since

$$
G \Phi=\frac{d \Phi}{d F_{1}} \cdot G F_{1}+\frac{d \Phi}{d F_{2}} \cdot G F_{2}+\frac{d \Phi}{d F_{3}^{\prime}} \cdot G F_{3}+\ldots
$$

we have to prove not only that each term of this expression is a reciprocant, but also that all of them have the same extraneous factor; otherwise their sum would not be a reciprocant.

Now, in

$$
\Phi=\Sigma A F_{1}^{\lambda_{1}} F_{2}^{\lambda_{2}} F_{3}^{\lambda_{3}} \cdots
$$

the extraneous factor for each term is by hypothesis $(-)^{\kappa} t^{\mu}$, so that the extraneous factor for each term of

$$
\frac{d \Phi}{d F_{1}}=\Sigma A \lambda_{1} F_{1}^{\lambda_{1}-1} F_{2}^{\lambda_{2}} F_{3}^{\lambda_{3}} \ldots
$$

is $(-)^{\kappa-\kappa_{1}} t^{\mu-\mu_{1}}$, and therefore $\frac{d \Phi}{d F_{1}}$ is a reciprocant. Also, $G F_{1}$ is a reciprocant whose extraneous factor is $(-)^{\kappa_{1}} t^{\mu_{1}+3}$. Hence $\frac{d \Phi}{d F_{1}} \cdot G F_{1}$ is a reciprocant having $(-)^{\kappa} t^{\mu+3}$ for extraneous factor, and in exactly the same way we see that every other term of $G \Phi$ is also a reciprocant with the same extraneous factor. 
Thus $G$, operating on any homogeneous reciprocant whose extraneous factor is $(-)^{\kappa} t^{\mu}$, generates another whose extraneous factor is $(-)^{\kappa} t^{\mu+3}$.

If, in the generator for mixed reciprocants,

$$
G=\left(2 b t-3 a^{2}\right) \partial_{a}+(2 c t-4 a b) \partial_{b}+(2 d t-5 a c) \partial_{c}+\ldots
$$

we write $\quad a=1.2 . a_{0}, b=1.2 .3 . a_{1}, c=1.2 .3 .4 . a_{2} \ldots$,

(that is, if we use the system of modified letters previously mentioned), its expression assumes a more elegant form. Substituting for $a, b, c, \ldots$ their values in terms of the modified letters, we have

$$
2 b t-3 a^{2}=2.1 .2 .3 a_{1} t-3(1,2)^{2} a_{0}^{2}=1.2^{2} \cdot 3\left(a_{1} t-a_{0}^{2}\right),
$$

and

$$
\partial_{a}=\frac{1}{1.2} \cdot \partial_{a_{0}}
$$

so that

$$
\left(2 b t-3 a^{2}\right) \partial_{a}=1.2 .3\left(a_{1} t-a_{0}^{2}\right) \partial_{a_{0}} \text {. }
$$

Again,

$$
(2 c t-4 a b)=1 \cdot 2^{2} \cdot 3 \cdot 4\left(a_{2} t-a_{0} a_{1}\right)
$$

and

$$
\partial_{b}=\frac{1}{1.2 .3} \partial_{a_{1}}
$$

so that

$$
(2 c t-4 a b) \partial_{b}=1.2 .4\left(a_{2} t-a_{0} a_{1}\right) \partial_{a_{1}} \text {. }
$$

Similarly,

$$
(2 d t-5 a c) \partial_{c}=1.2 .5\left(a_{3} t-a_{0} a_{2}\right) \partial_{a_{2}} \text {. }
$$

Thus the modified generator for mixed reciprocants is

1.2.3 $\left(a_{1} t-a_{0}^{2}\right) \partial_{a_{0}}+1.2 .4\left(a_{2} t-a_{0} a_{1}\right) \partial_{a_{1}}+1.2 .5\left(a_{3} t-a_{0} a_{2}\right) \partial_{a_{2}}+\ldots$, in which the general term is

$$
1.2(n+3)\left(a_{n+1} t-a_{0} a_{n}\right) \partial_{a_{n}} .
$$

The factor 1.2 may, of course, be rejected, and our modified generator may be written in the simple form

$$
3\left(a_{1} t-a_{0}^{2}\right) \partial_{a_{0}}+4\left(a_{2} t-a_{0} a_{1}\right) \partial_{a_{1}}+5\left(a_{3} t-a_{0} a_{2}\right) \partial_{a_{2}}+\ldots .
$$

Operating with this on the homogeneous reciprocant $F\left(t, a_{0}, a_{1}, a_{2}, \ldots\right)$, the result will be another homogeneous reciprocant of the same character as $F$. When we start with $a_{0}$ and make the reductions which, as we have seen, occur at every second step, we find a system of reduced educts corresponding in every particular with those formerly given, but expressed in terms of the modified letters $a_{0}, a_{1}, a_{2}, \ldots$ instead of $a, b, c, \ldots$. These are as follows :

$$
\begin{aligned}
& a_{0}, \\
& * a_{1} t-a_{0}{ }^{2}, \\
& 2 a_{2} t-5 a_{0} a_{1}, \\
& \text { *2 } 2 a_{3} t^{2}-6 a_{0} a_{2} t-3 a_{1}^{2} t+7 a_{0}^{2} a_{1}, \\
& 2 a_{4} t^{2}-7 a_{0} a_{3} t-7 a_{1} a_{2} t+8 a_{0}{ }^{2} a_{2}+11 a_{0} a_{1}{ }^{2}, \\
& \text { * } 14 a_{5} t^{3}-56 a_{0} a_{4} t^{2}-56 a_{1} a_{3} t^{2}-28 a_{2}{ }^{2} t^{2}+103 a_{0}^{2} a_{3} t+199 a_{0} a_{1} a_{2} t \\
& +33 a_{1}{ }^{3} t-88 a_{0}^{3} a_{2}-121 a_{0}{ }^{2} a_{1}{ }^{2} .
\end{aligned}
$$

* It will be observed that in the unreduced forms, marked with an asterisk, the sum of the numerical coefficients is zero. This is a direct consequence, as may be easily seen, of the form of the modified generator, in which the sum of the numerical coefficients in each term is also zero. 
It will be found on trial that these modified educts are obtained with greater ease and with less liability to error by a direct application of the generator

$$
3\left(a_{1} t-a_{0}^{2}\right) \partial_{a_{0}}+4\left(a_{2} t-a_{0} a_{1}\right) \partial_{a_{1}}+5\left(a_{3} t-a_{0} a_{2}\right) \partial_{a_{2}}+\ldots,
$$

than by making the substitution of $1.2 . a_{0}, 1.2 .3 . a_{1}, 1.2 .3 .4 . a_{2}, \ldots$ for $a, b, c, \ldots$ in the system of educts already given. For this reason the working by the former method is here performed, instead of being merely indicated.

From $a_{0}$ we obtain immediately

$$
a_{1} t-a_{0}^{2} .
$$

Operating on this with the generator, there results

$$
4 t\left(a_{2} t-a_{0} a_{1}\right)-6 a_{0}\left(a_{1} t-a_{0}^{2}\right)=4 a_{2} t^{2}-10 a_{0} a_{1} t+6 a_{0}^{3} .
$$

This, when reduced by removing its last term and dividing the others by $2 t$, gives

$$
2 a_{2} t-5 a_{0} a_{1}
$$

The next form is found from this by a simple operation, without subsequent reduction, and is therefore

$$
10 t\left(a_{3} t-a_{0} a_{2}\right)-20 a_{0}\left(a_{2} t-a_{0} a_{1}\right)-15 a_{1}\left(a_{1} t-a_{0}^{2}\right) .
$$

Or, collecting the terms and rejecting the numerical factor 5 ,

$$
2 a_{3} t^{2}-6 a_{0} a_{2} t-3 a_{1}^{2} t+7 a_{0}^{2} a_{1} .
$$

The operation of the generator on this gives

$$
\begin{aligned}
12 t^{2}\left(a_{4} t-a_{0} a_{3}\right)-30 a_{0} t\left(a_{3} t-a_{0} a_{2}\right)+4\left(7 a_{0}^{2}-6 a_{1} t\right) & \left(a_{2} t-a_{0} a_{1}\right) \\
& +3\left(14 a_{0} a_{1}-6 a_{2} t\right)\left(a_{1} t-a_{0}{ }^{2}\right) .
\end{aligned}
$$

The collection of terms and subsequent reduction is shown below :

$$
\begin{array}{ccccccc}
a_{4} t^{3} & a_{0} a_{3} t^{2} & a_{1} a_{2} t^{2} & a_{0}{ }^{2} a_{2} t & a_{0} a_{1}{ }^{2} t & a_{0}{ }^{3} a_{1} \\
12 & -12 & \cdot & \cdot & \cdot & \cdot \\
\cdot & -30 & \cdot & 30 & \cdot & \cdot \\
-14 a_{0}{ }^{2}\left(2 a_{2} t-5 a_{0} a_{1}\right)= & \cdot & -24 & 28 & 24 & -28 \\
\cdot & \cdot & -18 & 18 & 42 & -42 \\
\hline 12 & -42 & -42 & 76 & 66 & -70 \\
\hline 12 & -42 & -42 & 48 & 66 & .
\end{array}
$$

Removing the factor $6 t$, the reduced form is

$$
2 a_{4} t^{2}-7 a_{0} a_{3} t-7 a_{1} a_{2} t+8 a_{0}^{2} a_{2}+11 a_{0} a_{1}^{2} .
$$

Operating on this with the generator, we have

$$
\begin{aligned}
& 14 t^{2}\left(a_{5} t-a_{0} a_{4}\right)-42 a_{0} t\left(a_{4} t-a_{0} a_{3}\right)+5\left(8 a_{0}^{2}-7 a_{1} t\right)\left(a_{3} t-a_{0} a_{2}\right) \\
& \quad+4\left(22 a_{0} a_{1}-7 a_{2} t\right)\left(a_{2} t-a_{0} a_{1}\right)+3\left(11 a_{1}^{2}+16 a_{0} a_{2}-7 a_{3} t\right)\left(a_{1} t-a_{0}^{2}\right) \\
& \quad=14 a_{5} t^{3}-56 a_{0} a_{4} t^{2}-56 a_{1} a_{3} t^{2}-28 a_{2}{ }^{2} t^{2}+103 a_{0}{ }^{2} a_{3} t+199 a_{0} a_{1} a_{2} t \\
& \quad+33 a_{1}^{3} t-88 a_{0}^{3} a_{2}-121 a_{0}^{2} a_{1}^{2},
\end{aligned}
$$

which cannot be reduced in the same manner as the preceding form. 
To obtain a generator for passing from pure to pure reciprocants a process is employed similar to that which gave the generator for mixed reciprocants which we have just been using. I state the results before giving the proof, and then proceed to speak of generators in the theory of Invariants. The generator for pure reciprocants is

$$
\left(3 a c-4 b^{2}\right) \partial_{b}+(3 a d-5 b c) \partial_{c}+(3 a e-6 b d) \partial_{d}+\ldots ;
$$

or, expressed in terms of the modified letters,

$$
4\left(a_{0} a_{2}-a_{1}^{2}\right) \partial_{a_{1}}+5\left(a_{0} a_{3}-a_{1} a_{2}\right) \partial_{a_{2}}+6\left(a_{0} a_{4}-a_{1} a_{3}\right) \partial_{a_{3}}+\ldots .
$$

By operating with this on any pure reciprocant $R$, we generate another pure reciprocant of opposite character to that of $R$.

The connection between the two theories of Reciprocants and Invariants is so close, and these brother-and-sister theories throw so much light upon each other, that I began to inquire whether, in the latter, there did not exist a theory of Generators parallel to that of the former.

Fortunately, Mr Hammond was able to recall a correspondence in which Prof. Cayley had given such a theory, which he regarded, and justly, as an important invention. Its substance has been subsequently incorporated in the Quarterly Journal (Vol. xx. p. 212). It offers itself spontaneously in the Reciprocantive Theory; in the Invariantive one it calls for a distinct act of invention. Prof. Cayley has discovered two generators similar in form with those for reciprocants, and one of them strikingly so; in a letter to me he calls these $P$ and $Q$. As given by him,

$$
\begin{aligned}
& P=a b \partial_{a}+a c \partial_{b}+a d \partial_{c}+\ldots-i b, \\
& Q=a c \partial_{b}+2 a d \partial_{c}+\ldots-2 w b,
\end{aligned}
$$

where $i$ is the degree and $w$ the weight, the weights of $a, b, c, d, \ldots$ being taken to be $0,1,2,3, \ldots$ (I supply the $a$ which Cayley turns into unity.) As an example he takes the "Invariant" $a^{2} d-3 a b c+2 b^{3}=I$, suppose. We have then

$$
\begin{aligned}
P I & =\left(a b \partial_{a}+a c \partial_{b}+a d \partial_{c}+a e \partial_{d}-3 b\right) I \\
& =a b(2 a d-3 b c)+a c\left(-3 a c+6 b^{2}\right)-3 a^{2} b d+a^{3} e-3 b\left(a^{2} d-3 a b c+2 b^{3}\right) \\
& =a^{3} e-4 a^{2} b d-3 a^{2} c^{2}+12 a b^{2} c-6 b^{4} \\
& =a^{2}\left(a e-4 b d+3 c^{2}\right)-6\left(a c-b^{2}\right)^{2},
\end{aligned}
$$

and $Q I=\left(a c \partial_{b}+2 a d \partial_{c}+3 a e \partial_{d}-6 b\right) I$

$$
\begin{aligned}
& =a c\left(-3 a c+6 b^{2}\right)-6 a^{2} b d+3 a^{3} e-6 b\left(a^{2} d-3 a b c+2 b^{3}\right) \\
& =3 a^{3} e-12 a^{2} b d-3 a^{2} c^{2}+24 a b^{2} c-12 b^{4} \\
& =3 a^{2}\left(a e-4 b d+3 c^{2}\right)-12\left(a c-b^{2}\right)^{2} .
\end{aligned}
$$

$P$ and $Q$ may be transformed by means of Euler's equation and the similar one for isobaric functions, which enable us to write

and

$$
i=a \partial_{a}+b \partial_{b}+c \partial_{c}+d \partial_{d}+\ldots
$$

$$
w=\quad b \partial_{b}+2 c \partial_{c}+3 d \partial_{d}+\ldots ;
$$


$P$ thus becomes

$$
\begin{gathered}
a b \partial_{a}+a c \partial_{b}+a d \partial_{c}+a e \partial_{d}+\ldots \\
-a b \partial_{a}-b^{2} \partial_{b}-b c \partial_{c}-b d \partial_{d}-\ldots \\
=\left(a c-b^{2}\right) \partial_{b}+(a d-b c) \partial_{c}+(a e-b d) \partial_{d}+\ldots
\end{gathered}
$$

the same in form as either of our generators, except that the arithmetical coefficients are all made units; $a, b, c, \ldots$ taking the place of the $t, a, b, \ldots$ of the generator for mixed reciprocants.

In like manner, $Q$ becomes

$$
\left(a c-2 b^{2}\right) \partial_{b}+2(a d-2 b c) \partial_{c}+3(a e-2 b d) \partial_{d}+\ldots,
$$

where the arithmetical series $1,2,3, \ldots$ takes the place of $3,4,5, \ldots$ or of $4,5,6, \ldots$ in the two Reciprocant Generators.

The effect of $P$ and of $Q$ is obviously to raise the degree and the weight of the operand $I$ each by one unit. But if we take $R=\frac{1}{a}(2 w P-i Q)$, the terms in Cayley's original formulae containing $b$ cancel, so that $2 w P-i Q$ divides out by $a$ and the weight is raised one unit without the degree being affected. This is mentioned in the Quarterly Journal (loc. cit.); but it may also be remarked that when $I$ is a satisfied invariant, it is annihilated by the operation of $R$; when the invariant is unsatisfied, each of the three operators $P, Q$ and $R$ increases its extent by an unit, that is, introduces an additional letter. For let $j$ denote the extent, then, writing $a_{0}, a_{1}, a_{2}, \ldots a_{j}$ for $a, b, c, \ldots$, we have

$$
\begin{aligned}
& P=a_{0} a_{1} \partial_{a_{0}}+a_{0} a_{2} \partial_{a_{1}}+\ldots+a_{0} a_{j+1} \partial_{a_{j}}-i a_{1} \\
& Q=\quad a_{0} a_{2} \partial_{a_{1}}+2 a_{0} a_{3} \partial_{a_{2}}+\ldots+j a_{0} a_{j+1} \partial_{a_{j}}-2 w a_{1}
\end{aligned}
$$

whence we find

$$
\begin{aligned}
R & =\frac{1}{a_{0}}(2 w P-i Q) \\
& =2 w a_{1} \partial_{a_{0}}+(2 w-i) a_{2} \partial_{a_{1}}+\ldots+(2 w-i j+i) a_{j} \partial_{a_{j-1}}+(2 w-i j) a_{j+1} \partial_{a_{j}} .
\end{aligned}
$$

But for a satisfied invariant

$$
2 w=i j
$$

and substituting this value for $2 w$ in the above expression for $R$, it becomes

$$
i\left\{j a_{1} \partial_{a_{0}}+(j-1) a_{2} \partial_{a_{1}}+\ldots+a_{j} \partial_{a_{j-1}}\right\}
$$

which, as is well known, annihilates any satisfied invariant. 


\section{LECTURE V.}

It will be desirable to fill up some of the previous investigations by discussing some points in them that have not yet received our consideration.

There may be some to whom it may appear tedious to watch the complete exposition of the algebraical part of the Theory, who are impatient to rush on to its applications. But it is my duty to consider what may be expected to be most useful to the great majority of the class, and for that purpose to make the ground sure under our feet as I proceed. To the greater number it will, I think, be of advantage to have their memories refreshed on the kindred subject of invariants, and probably made acquainted with some important points of that theory which are new to them.

I confess that, to myself, the contemplation of this relationship-the spectacle of a new continent rising from the waters, resembling yet different from the old, familiar one-is a principal source of interest arising out of the new theory. I do not regard Mathematics as a science purely of calculation, but one of ideas, and as the embodiment of a Philosophy. An eminent colleague of mine, in a public lecture in this University, magnifying the importance of classical over mathematical studies, referred to a great mathematician as one who might possibly know every foot of distance between the earth and the moon; and when I was a member, at Woolwich, of the Government Committee of Inventions, one of my colleagues, appealing to me to answer some question as to the number of cubic inches in a pipe, expressed his surprise that I was not prepared with an immediate answer, and said he had supposed that I had all the tables of weights and measures at my fingers' ends.

I hope that in any class which I may have the pleasure of conducting in this University, other ideas will prevail as to the true scope of mathematical science as a branch of liberal learning; and it will be my endeavour to regulate the pace in a manner which seems to me most conducive to real progress in the order of ideas and philosophical contemplation, thus bringing our noble science into harmony and in a line with the prevailing tone and studies of this University. Faraday, at the end of his experimental lectures, was accustomed to say-I have myself heard him do so-"We will now leave that to the calculators." So long as we are content to be regarded as mere calculators we shall be the Pariahs of the University, living here on sufferance, instead of being regarded, as is our right and privilege, as the real leaders and pioneers of thought in it. 
That Cayley's two operators, which have been called $P$ and $Q$, are in fact generators, may be proved as follows + :

Let

and

$$
\begin{aligned}
& \Omega=a \partial_{b}+2 b \partial_{c}+3 c \partial_{d}+4 d \partial_{e}+\ldots, \\
& \Theta=a\left(\lambda b \partial_{a}+\mu c \partial_{b}+\nu d \partial_{c}+\ldots\right)-\kappa b,
\end{aligned}
$$

where $\kappa, \lambda, \mu, \nu, \ldots$ are numbers.

When $\kappa$ is the degree of the operand, and $\lambda=\mu=\nu=\ldots=1$, the operator $\Theta$ is identical with $P$; but $\Theta$ is identical with $Q$ when $\kappa$ is twice the weight of the operand and $\lambda=0, \mu=1, \nu=2, \ldots$.

If now we use * to signify the act of pure differential operation, it is obvious that

so that

$$
\begin{aligned}
& \Omega \Theta=(\Omega \times \Theta)+(\Omega * \Theta), \\
& \Theta \Omega=(\Omega \times \Theta)+(\Theta * \Omega),
\end{aligned}
$$

But since

$$
\Omega \Theta-\Theta \Omega=(\Omega * \Theta)-(\Theta * \Omega) \text {. }
$$

we have

$$
\Omega a=0, \Omega b=a, \quad \Omega c=2 b, \ldots
$$

and

$$
\Omega * \Theta=a\left(\lambda a \partial_{a}+2 \mu b \partial_{b}+3 \nu c \partial_{c}+\ldots-\kappa\right)
$$

Hence $\quad \Omega \Theta-\Theta \Omega=a\left\{\lambda a \partial_{a}+(2 \mu-\lambda) b \partial_{b}+(3 \nu-2 \mu) c \partial_{c}+\ldots-\kappa\right\} ;$
now if the operand $I$ be any invariant (satisfied or unsatisfied), we have $\Omega I=0$,

$\Theta * \Omega=a\left(\lambda b \partial_{b}+2 \mu c \partial_{c}+3 \nu d \partial_{d}+\ldots\right)$. and therefore $\Theta \Omega I=0$; so that we find

$$
\Omega \Theta I=a\left\{\lambda a \partial_{a}+(2 \mu-\lambda) b \partial_{b}+(3 \nu-2 \mu) c \partial_{c}+\ldots-\kappa\right\} I .
$$

If in this we write $\lambda=\mu=\nu=\ldots=1$, and $\kappa=i$, where $i$ is the degree of the operand, $\Theta$ becomes $P$ and we have

$$
\Omega P I=a\left(a \partial_{a}+b \partial_{b}+c \partial_{c}+\ldots-i\right) I .
$$

But, by Euler's theorem, the right-hand side of this vanishes, and therefore

$$
\Omega P I=0 .
$$

Similarly, by means of the corresponding theorem for isobaric functions, we may prove that

$$
\Omega Q I=0 .
$$

For if, in the general formula, we write $\lambda=0, \mu=1, \nu=2, \ldots$ and $\kappa=2 w$, where $w$ is the weight of the operand, we find

$$
\Omega Q I=a\left(2 b \partial_{b}+4 c \partial_{c}+6 d \partial_{d}+\ldots-2 w\right) I=0 .
$$

Thus, when $\Theta$ stands either for $P$ or for $Q$, it is either an annihilator or a generator (that is, $\Theta I$ is either identically zero or else an invariant). But if $l$ be the most advanced, or say the radical letter of $I$, no term of $m \partial_{l} I$ can cancel with any other term of $\Theta I$; and since, for this reason, $\Theta I$ cannot vanish identically, it must be an invariant, and the operators $P$ and $Q$ must be generators.

† In the Quarterly Journal (Vol. xx. p. 212) Prof. Cayley only considers a special example, and has not given the proof of the general theorem. 
The generators previously given for reciprocants also possess this property of introducing a fresh radical letter at each step. The radical letter, on its first introduction, enters in the first degree only, and in the case of the educts of $\log t$, whose values have been calculated, its multiplier is seen to be a power of $t$. The form of the generator for mixed reciprocants

$$
3\left(a_{1} t-a_{0}^{2}\right) \partial_{a_{0}}+4\left(a_{2} t-a_{0} a_{1}\right) \partial_{a_{1}}+\ldots+(n+3)\left(a_{n+1} t-a_{0} a_{n}\right) \partial_{a_{n}}
$$

shows this, or it may be seen by considering the successive values of

$$
\left(\frac{1}{\sqrt{ } t} \cdot \frac{d}{d x}\right)^{i} \log t
$$

For let $\frac{F\left(t, a_{0}, a_{1}, a_{2}, \ldots\right)}{t^{\lambda}}$ denote this expression, and let its radical letter be $a_{n}$; then, on differentiating again with respect to $x$, the new letter introduced arises solely from a term in the numerator

$$
\frac{d}{d a_{n}} F\left(t, a_{0}, a_{1}, a_{2}, \ldots a_{n}\right) \cdot \frac{d a_{n}}{d x} .
$$

But $\quad a_{n}=\frac{d^{n} y}{d x^{n}} \div 2.3 \ldots n+2 ;$ so that $\frac{d a_{n}}{d x}=(n+3) a_{n+1}$.

Hence, if when $a_{n}$ is the radical letter, it occurs in the first degree only and multiplied by a power of $t$, it follows that, since $\frac{d F}{d a_{n}}$ will be a power of $t$, the derived expression which contains the radical letter $a_{n+1}$ will contain it in the first degree only and multiplied by a power of $t$. And since this is true for the case $i=1$, when $\frac{1}{\sqrt{ } t} \cdot \frac{d}{d x} \log t=\frac{a_{0}}{t^{\frac{3}{2}}}$, it is true universally.

Observe that for $i=1,2,3, \ldots$ the radical letter is $a_{0}, a_{1}, a_{2}, \ldots$ respectively.

It will be remembered that $\left(\frac{1}{\sqrt{ } t} \cdot \frac{d}{d x}\right)^{i} \log t$ is an absolute reciprocant. It may be called the $i$ th absolute educt, to distinguish it from the rational integral educts $E_{1}, E_{2}, E_{3}, \ldots$ whose values have already been calculated.

Let $R\left(t, a_{0}, a_{1}, a_{2}, \ldots a_{n}\right)$ be any homogeneous rational integral reciprocant, and let the educts be $A_{0}, A_{1}, A_{2}, \ldots A_{n}$; then obviously

$a_{n}$ may be expressed rationally in terms of $A_{n}$ and $a_{n-1}, a_{n-2}, \ldots a_{0}, t$,

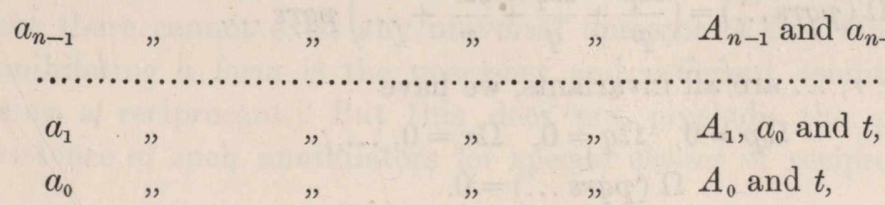

where observe that the denominators in these expressions are all powers of $t$. Hence, by successive substitutions, $R\left(t, a_{0}, a_{1}, \ldots a_{n}\right)$ may be expressed 
rationally in terms of $A_{n}, \ldots A_{1}, A_{0}$, and $t$. Thus any rational integral homogeneous reciprocant is a rational function of educts, and is of the form $\frac{E}{t^{0}}$, where $E$ is a rational integral function of the educts.

Does not this prove too much, it may be asked, namely, that any function $F$ of the letters is a rational function of the educts, which are themselves reciprocants, and will therefore be a reciprocant? But this is not so; for observe that although $F$ will be expressed as a sum of products of educts, such products will not in general be all of the same character, and their linear combination will be an illicit one, such as is seen in the illicit combination of $a_{0}^{2}$ with the Schwarzian $\left(a_{1} t-a_{0}^{2}\right)$.

We have seen that by differentiating an absolute reciprocant, or by the use of a generator, we obtain a fresh reciprocant. But there are other methods of finding reciprocants; as, for example, if the transform of

is

$$
\phi(t, a, b, c, \ldots)
$$

is

$$
\psi(\tau, \alpha, \beta, \gamma, \ldots)
$$

that is, if

$$
\phi(t, a, b, c, \ldots)=\psi(\tau, \alpha, \beta, \gamma, \ldots)
$$

then

$$
\psi(t, a, b, c, \ldots)=\phi(\tau, \alpha, \beta, \gamma, \ldots) .
$$

Whence, by multiplication,

$$
\phi(t, a, b, c, \ldots) \psi(t, a, b, c, \ldots)=\phi(\tau, \alpha, \beta, \gamma, \ldots) \psi(\tau, \alpha, \beta, \gamma, \ldots) .
$$

Thus $\phi . \psi$ is a reciprocant, and, moreover, an absolute one of even character, although neither $\phi$, which is a perfectly arbitrary function, nor $\psi$, its transform, is a reciprocant.

Herein a mixed reciprocant differs from an invariant, which cannot be resolved into non-invariantive factors. It is worth while to give a proof of this proposition; but first I prove its converse, that if $p, q, r, \ldots$ are all invariants, their product must be so too. This is an immediate consequence of the well-known theorem that

$$
\Omega I=0
$$

is the necessary and sufficient condition that $I$ may be an invariant where, as usual, $\Omega$ is the operator

$$
a \partial_{b}+2 b \partial_{c}+3 c \partial_{d}+\ldots
$$

and the word invariant has been used in the same extended sense as formerly.

$$
\text { For } \quad \Omega(p q r s \ldots)=\left(\frac{\Omega p}{p}+\frac{\Omega q}{q}+\frac{\Omega r}{r}+\ldots\right) \text { pqrs .... }
$$

But since $p, q, r, \ldots$ are all invariants, we have

and therefore

$$
\Omega p=0, \quad \Omega q=0, \quad \Omega r=0, \ldots
$$

Next, suppose that

$$
\begin{gathered}
\Omega(\text { pqrs } \ldots)=0 . \\
I=P_{1} Q_{1},
\end{gathered}
$$

where $I$ is but $Q_{1}$ is not an invariant. 
To meet the case in which $P_{1}$ and $Q_{1}$ are not prime to one another, $Q_{1}$, if resolved into its factors, must contain one $Q^{i}$ where $Q$ is not an invariant.

Suppose that $P_{1}$ contains $Q^{j}$, and let $i+j=k$; then we may write

$$
I=P Q^{k}
$$

where $P$ is prime to $Q$. But since $I$ is an invariant by hypothesis,

$$
\Omega I=0,
$$

and therefore,

$$
\begin{gathered}
Q^{k} \Omega P+k P Q^{k-1} \Omega Q=0 \\
\frac{Q}{P}=-k \frac{\Omega Q}{\Omega P} .
\end{gathered}
$$

or,

Now $P$ is prime to $Q$, so that the fraction $\frac{Q}{P}$ is in its lowest terms; therefore $\Omega Q$ contains $Q$; but this is impossible, for the weight of $\Omega Q$ is less than that of $Q$. Hence $I$ cannot contain any non-invariantive factor $Q_{1}$.

All this will be equally true for a general function $J$ annihilated by any operator $\Omega$ which is linear in the differential operators $\partial_{a}, \partial_{b}, \partial_{c}, \ldots$ no matter what its degree in the letters $a, b, c, \ldots$ themselves; that is, we shall still have

and

$$
\begin{aligned}
& J=P Q^{k} \\
& \frac{Q}{P}=-k \frac{\Omega Q}{\Omega P},
\end{aligned}
$$

where $P$ and $Q$ are prime to each other, and, as before, $\Omega Q$ will contain $Q$ as a factor. But if $\Omega$ is an operator which diminishes either the degree or the weight, $\Omega Q$ is either of lower degree or of lower weight than $Q$, and so cannot contain it as a factor. Hence $J$ cannot contain a factor $Q$ not subject to annihilation by $\Omega$.

If, however, $\Omega$ does not diminish either the degree or the weight, it may be objected that $\Omega Q$ might conceivably contain the factor $Q$; and were it so, there would be nothing to show the impossibility, in this case, of a function $J$ subject to annihilation by $\Omega$ containing a factor $Q$, which is not so. But quaere: Is it possible, when $J$ is a general homogeneous and isobaric function of $a, b, c, \ldots$, for $\Omega J$ to contain $J$ and at the same time the quotient to be other than a number*? Valde dubitor. But I reserve the point. Setting aside this doubtful case, and considering only such linear partial differential operators as diminish either the degree or the weight of the operand, we see that there cannot exist any universal operator of this kind whose effect in annihilating a form is the necessary and sufficient condition of that form being a reciprocant. But this does not preclude the possibility of the existence of such annihilators for special classes of reciprocants, and in fact

* If $\Omega=p a \partial_{a}+q b \partial_{b}+r c \partial_{c}+\ldots$, where $p, q, r, \ldots$ are in Arithmetical Progression, $\frac{\Omega J}{J}$ is a number; but then $\Omega$ could not be an annihilator. 
(as we have already stated and shall hereafter prove) Pure Reciprocants are definable by means of the Partial Differential Annihilator

$$
V=4 \cdot \frac{a_{0}^{2}}{2} \partial_{a_{1}}+5 a_{0} a_{1} \partial_{a_{2}}+6\left(a_{0} a_{2}+\frac{a_{1}^{2}}{2}\right) \partial_{a_{3}}+\ldots
$$

which is linear in the differential operators, and diminishes the weight.

The generator for mixed reciprocants, which we have called $G$, will not assist us in obtaining pure reciprocants, but generates a mixed reciprocant in every case, even when the one we start with is pure. Thus, starting with the pure reciprocant $R$, our formula

$$
G R=\left\{3\left(a_{1} t-a_{0}^{2}\right) \partial_{a_{0}}+4\left(a_{2} t-a_{0} a_{1}\right) \partial_{a_{1}}+5\left(a_{3} t-a_{0} a_{2}\right) \partial_{a_{2}}+\ldots\right\} R
$$

may be written thus

$$
\begin{aligned}
G R & =t\left(3 a_{1} \partial_{a_{0}}+4 a_{2} \partial_{a_{1}}+5 a_{3} \partial_{a_{2}}+\ldots\right) R \\
& -a_{0}\left(3 a_{0} \partial_{a_{0}}+4 a_{1} \partial_{a_{1}}+5 a_{2} \partial_{a_{2}}+\ldots\right) R .
\end{aligned}
$$

Here $R$ being pure, that is, a function of $a_{0}, a_{1}, a_{2}, \ldots$ (without $t$ ), we see that

$$
\begin{aligned}
\left(3 a_{0} \partial_{a_{0}}+\right. & \left.4 a_{1} \partial_{a_{1}}+5 a_{2} \partial_{a_{2}}+6 a_{3} \partial_{a_{3}}+\ldots\right) R \\
& =3\left(a_{0} \partial_{a_{0}}+a_{1} \partial_{a_{1}}+a_{2} \partial_{a_{2}}+\ldots\right) R \\
& +\left(a_{1} \partial_{a_{1}}+2 a_{2} \partial_{a_{2}}+3 a_{3} \partial_{a_{3}}+\ldots\right) R \\
& =(3 i+w) R
\end{aligned}
$$

where $i$ is the degree and $w$ the weight of $R$. Hence

$$
G R=t\left(3 a_{1} \partial_{a_{0}}+4 a_{2} \partial_{a_{1}}+5 a_{3} \partial_{a_{2}}+\ldots\right) R-(3 i+w) a_{0} R
$$

where it should be noticed that $a_{0} R$ is of opposite character to $R$ (for $a_{0}$ is of odd character), while $G R$ has been proved to be of the same character as $R$. Thus we cannot infer that $t\left(3 a_{1} \partial_{a_{0}}+4 a_{2} \partial_{a_{1}}+5 a_{3} \partial_{a_{2}}+\ldots\right) R$ is a reciprocant. The mixed reciprocant $G R$ cannot therefore be resolved into the sum of two terms, one of which is a pure reciprocant and the other a pure reciprocant multiplied by $t$.

\section{LECTURE VI.}

Before proceeding to prove that, as was stated in anticipation in Lecture IV, the operator

$$
\left(3 a c-4 b^{2}\right) \partial_{b}+(3 a d-5 b c) \partial_{c}+(3 a e-6 b d) \partial_{d}+\ldots
$$

or, when the modified letters are used,

$$
4\left(a_{0} a_{2}-a_{1}^{2}\right) \partial_{a_{1}}+5\left(a_{0} a_{3}-a_{1} a_{2}\right) \partial_{a_{2}}+6\left(a_{0} a_{4}-a_{1} a_{3}\right) \partial_{a_{3}}+\ldots
$$

will serve to generate a pure reciprocant from a pure one, it may be useful to briefly recapitulate what has been said concerning the character and 
characteristic of reciprocants. It will be remembered that the extraneous factor of any rational integral reciprocant is of the form $(-)^{\kappa} t^{\mu}$, that the character is determined by the parity (oddness or evenness) of $\kappa$, and that $\mu$ is what has been called the characteristic.

For homogeneous reciprocants it has been proved that $\mu=3 i+w$, where $i$ is the degree of the reciprocant and $w$ its weight, the weights of the letters $t, a, b, c, \ldots$ being taken to be $-1,0,1,2, \ldots$ respectively. The character is odd or even according as the number of letters other than $t$ in the principal term or terms is odd or even. By a principal term is to be understood one in which $t$ is contained the greatest number of times. So that, in other words, the character is governed by the parity of the smallest number of non- $t$ letters that can be found in any term. For pure reciprocants, there being no $t$ in any term, the character is determined by the parity of the number of letters in any one term.

Let $R$ be any pure reciprocant, and suppose its characteristic to be $\mu$; then $\frac{R}{t^{\frac{\mu}{2}}}$ is an absolute reciprocant. If, however, we differentiate this with respect to $x$, and thus obtain another reciprocant, the resulting form will not be pure, for its numerator will be identical with the form obtained by the direct operation on $R$ of the generator for mixed reciprocants, and its denominator will be a power of $t$. But, remembering that $\frac{a}{t^{\frac{3}{2}}}$, and therefore $\frac{a^{\frac{\mu}{3}}}{t^{\frac{\mu}{2}}}$, is an absolute reciprocant, we see that $\frac{R}{a^{\frac{\mu}{3}}}$, which is the quotient of the two absolute reciprocants $\frac{R}{t^{\frac{\mu}{2}}}$ and $\frac{a^{\frac{\mu}{3}}}{t^{\frac{\mu}{2}}}$, is so also. Hence $\frac{d}{d x}\left(\frac{R}{a^{\frac{\mu}{3}}}\right)$ is a reciprocant, and, since it no longer contains $t$, a pure one. Now,

$$
\frac{d}{d x}\left(\frac{R}{a^{\frac{\mu}{3}}}\right)=\frac{a \frac{d R}{d x}-\frac{\mu}{3} \cdot b R}{a^{\frac{\mu}{3}+1}}
$$

remains a reciprocant when multiplied by any power of the reciprocant $a$. Hence the numerator of this expression, or

$$
\left(3 a \frac{d}{d x}-\mu b\right) R
$$

is a reciprocant. The general value of $\frac{d}{d x}$ has been seen to be

$$
a \partial_{t}+b \partial_{a}+c \partial_{b}+d \partial_{c}+\ldots
$$

but, since $R$ is supposed to be pure, $\partial_{t} R=0$. 
We may therefore, in $3 a \frac{d}{d x}-\mu b$, replace $\frac{d}{d x}$ by

$$
b \partial_{a}+c \partial_{b}+d \partial_{c}+e \partial_{d}+\ldots .
$$

Now, remembering that $\mu=3 i+w$, and that by Euler's theorem and the similar one for isobaric functions

and

$$
\begin{aligned}
& i=a \partial_{a}+b \partial_{b}+c \partial_{c}+d \partial_{d}+\ldots \\
& w=\quad b \partial_{b}+2 c \partial_{c}+3 d \partial_{d}+\ldots
\end{aligned}
$$

we see that $\mu$ is equivalent to

$$
3 a \partial_{a}+4 b \partial_{b}+5 c \partial_{c}+6 d \partial_{d}+\ldots
$$

Hence, $\quad 3 a \frac{d}{d x}-\mu b=3 a\left(b \partial_{a}+c \partial_{b}+d \partial_{c}+e \partial_{d}+\ldots\right)$

$$
\begin{aligned}
& -b\left(3 a \partial_{a}+4 b \partial_{b}+5 c \partial_{c}+6 d \partial_{d}+\ldots\right) \\
& =\left(3 a c-4 b^{2}\right) \partial_{b}+(3 a d-5 b c) \partial_{c}+(3 a e-6 b d) \partial_{d}+\ldots
\end{aligned}
$$

Thus, if $R$ be any pure reciprocant,

$$
\left\{\left(3 a c-4 b^{2}\right) \partial_{b}+(3 a d-5 b c) \partial_{c}+(3 a e-6 b d) \partial_{d}+\ldots\right\} R
$$

is also a pure reciprocant. If the type of $R$ be $w ; i, j$, that of the form derived from it will clearly be $w+1 ; i+1, j+1$. Its character (which, for pure reciprocants, depends solely on the degree) will therefore be opposite to that of $R$, and its characteristic will be $\mu+4$, that of $R$ being $\mu$.

Beginning with the form $3 a c-5 b^{2}$, which was given as an example in Lecture II, a series of pure "educts" may be obtained by the repeated use of the above generator; and it will be noticed that the successive educts thus formed are alternately of even and odd character, whereas those previously given, namely, $a, 2 b t-3 a^{2} \ldots$, were all negative. A reduction similar to that which formerly took place when the generator for mixed reciprocants was used, may be effected at each second step in the present case. For, since the characteristic of $\left(3 a \frac{d}{d x}-\mu b\right) R$ is $\mu+4$, the next operation will give

$$
\left(3 a \frac{d}{d x}-(\mu+4) b\right)\left(3 a \frac{d}{d x}-\mu b\right) R .
$$

Performing the indicated differentiations, this becomes

$$
\begin{aligned}
& 3 a \frac{d}{d x}\left(3 a \frac{d R}{d x}-\mu b R\right)-3(\mu+4) a b \frac{d R}{d x}+\mu(\mu+4) b^{2} R \\
= & 9 a^{2} \frac{d^{2} R}{d x^{2}}+9 a b \frac{d R}{d x}-3 \mu a b \frac{d R}{d x}-3 \mu a c R-3(\mu+4) a b \frac{d R}{d x}+\mu(\mu+4) b^{2} R \\
= & 9 a^{2} \frac{d^{2} R}{d x^{2}}-3(2 \mu+1) a b \frac{d R}{d x}-3 \mu a c R+\mu(\mu+4) b^{2} R .
\end{aligned}
$$


Adding $\mu(\mu+4)\left(3 a c-5 b^{2}\right) R$ to 5 times the above expression, we obtain

$$
45 a^{2} \frac{d^{2} R}{d x^{2}}-15(2 \mu+1) a b \frac{d R}{d x}+3 \mu(\mu-1) a c R
$$

which, when divided by $3 a$, gives the pure reciprocant

$$
15 a \frac{d^{2} R}{d x^{2}}-5(2 \mu+1) b \frac{d R}{d x}+\mu(\mu-1) c R
$$

This form is one degree lower than the second educt from $R$, the depression of degree being due to the removal of a factor $a$ by division.

When the modified letters $a_{0}, a_{1}, a_{2}, a_{3}, \ldots$ are used, the generator

$$
\left(3 a c-4 b^{2}\right) \partial_{b}+(3 a d-5 b c) \partial_{c}+(3 a e-6 b d) \partial_{d}+\ldots
$$

is easily transformed by writing in it

$$
a=2 a_{0}, \quad b=2.3 . a_{1}, \quad c=2.3 .4 . a_{2}, \quad d=2.3 .4 .5 \quad a_{3} \ldots,
$$

and consequently

$$
\partial_{b}=\frac{\partial_{a_{1}}}{2.3}, \quad \partial_{c}=\frac{\partial_{a_{2}}}{2.3 .4}, \quad \partial_{d}=\frac{\partial_{a_{3}}}{2.3 .4 .5} \ldots
$$

when it becomes

$$
\begin{aligned}
\frac{2^{2} \cdot 3^{2} \cdot 4}{2.3}\left(a_{0} a_{2}-a_{1}^{2}\right) \partial_{a_{1}}+\frac{2^{2} \cdot 3^{2} \cdot 4 \cdot 5}{2.3 .4}\left(a_{0} a_{3}-\right. & \left.a_{1} a_{2}\right) \partial_{a_{2}} \\
& +\frac{2^{2} \cdot 3^{2} \cdot 4 \cdot 5 \cdot 6}{2.3 .4 \cdot 5}\left(a_{0} a_{4}-a_{1} a_{3}\right) \partial_{a_{3}}+\ldots .
\end{aligned}
$$

Dividing each term of this by 2.3 , and writing the numerical coefficients in their simplest form, we have

$$
4\left(a_{0} a_{2}-a_{1}^{2}\right) \partial_{a_{1}}+5\left(a_{0} a_{3}-a_{1} a_{2}\right) \partial_{a_{2}}+6\left(a_{0} a_{4}-a_{1} a_{3}\right) \partial_{a_{3}}+\ldots
$$

which is the modified generator previously mentioned.

The generators formerly used in the theory of mixed reciprocants were

$$
\left(2 t b-3 a^{2}\right) \partial_{a}+(2 t c-4 a b) \partial_{b}+(2 t d-5 a c) \partial_{c}+\ldots
$$

and

$$
3\left(t a_{1}-a_{0}^{2}\right) \partial_{a_{0}}+4\left(t a_{2}-a_{0} a_{1}\right) \partial_{a_{1}}+5\left(t a_{3}-a_{0} a_{2}\right) \partial_{a_{2}}+\ldots
$$

The memory will be assisted in retaining these formulae if we observe that (1) is obtainable from (3), or (2) from (4), by increasing at the same time each numerical coefficient and the weight of each letter by unity.

It will, I think, be instructive to see how the form $3 a c-5 b^{2}$ was found originally by combining mixed reciprocants. The degree alone of a pure reciprocant suffices, as we have seen, to determine its character; but when we are dealing with mixed reciprocants their character does not depend either on the degree or the weight, so that we require a notation to discriminate between forms of the same degree-weight, but of opposite character. In what follows, $(+)$ placed before any form signifies that it is a reciprocant of even character, while (-) signifies that its character is odd. 
I have previously given the three odd reciprocants

$$
\begin{aligned}
& \text { (-) } a \\
& \text { (-) } 2 b t-3 a^{2} \\
& \text { (-) } c t-5 a b .
\end{aligned}
$$

From these we obtain even reciprocants; thus the product of (A) and (C) is

and the square of $(\mathrm{B})$ is

$$
\text { (+) } a c t-5 a^{2} b
$$

$$
\text { (+) } 4 b^{2} t^{2}-12 a^{2} b t+9 a^{4}
$$

After subtracting the even reciprocant $9 a^{4}$ from this, we may remove the factor $4 t$ from the remainder without thereby affecting its character. These reductions give

$$
\text { (+) } b^{2} t-3 a^{2} b
$$

which may be combined with the even reciprocant (D) in such a manner that the combination contains a factor $t$. In fact,

$$
3\left(a c t-5 a^{2} b\right)-5\left(b^{2} t-3 a^{2} b\right)=\left(3 a c-5 b^{2}\right) t,
$$

so that a legitimate combination of mixed reciprocants can be made to give the pure one

$$
3 a c-5 b^{2}
$$

Similarly we might find the known form

$$
9 a^{2} d-45 a b c+40 b^{3}
$$

which equated to zero expresses Sextactic Contact at a point $x, y$. But it is more readily obtained by operating with the generator on $3 a c-5 b^{2}$; thus,

$$
\begin{aligned}
\left\{\left(3 a c-4 b^{2}\right) \partial_{b}+(3 a d-5 b c) \partial_{c}\right\}\left(3 a c-5 b^{2}\right) & =-10 b\left(3 a c-4 b^{2}\right)+3 a(3 a d-5 b c) \\
- & =9 a^{2} d-45 a b c+40 b^{3} .
\end{aligned}
$$

An orthogonal reciprocant may be defined as a mixed reciprocant whose form remains invariable (save as to the acquisition of an extraneous factor when the reciprocant is not absolute) when any orthogonal substitution is impressed on the variables $x$ and $y$. Concerning such reciprocants, we have the very beautiful theorem : If $R$ and $\frac{d R}{d t}$ are both of them reciprocants, then $R$ is an orthogonal reciprocant.

For suppose $R$ to be an absolute reciprocant; that is, let

$$
R=q R^{\prime}(q= \pm 1)
$$

where $R$ is a function of $t, a, b, c, \ldots$ and $R^{\prime}$ the same function of $\tau, \alpha, \beta, \gamma, \ldots$; then, denoting by $\Delta R$ the variation of $R$ due to the variation of $y$ by $\epsilon x$, and by $D R$ the variation of $R$ due to the variation of $x$ by $-\epsilon y$, we have

$$
\Delta R=\epsilon \frac{d R}{d t}
$$


For the variation of $t$ is $\epsilon$ and the variations of $a, b, c, \ldots$ vanish. Similarly

$$
D R^{\prime}=-\epsilon \frac{d R^{\prime}}{d \tau}
$$

Now, since

$$
R=q R^{\prime},
$$

$$
D R=q D R^{\prime}=-\epsilon q \frac{d R^{\prime}}{d \tau}
$$

therefore

$$
D R+\Delta R=\epsilon\left(\frac{d R}{d t}-q \frac{d R^{\prime}}{d \tau}\right)
$$

that is, the total variation of $R$ (due to the change of $x$ into $x-\epsilon y$ and of $y$ into $y+\epsilon x)$ vanishes if

$$
\frac{d R}{d t}=q \frac{d R^{\prime}}{d \tau} .
$$

Hence, if $R$ be an absolute orthogonal reciprocant, $\frac{d R}{d t}$ is also an absolute reciprocant (though it is not orthogonal) of the same character as $R$.

If $R$ be not absolute, suppose its characteristic to be $\mu$; then it can be made absolute by dividing it by $a^{\frac{\mu}{3}}$. The application of the foregoing method of variations will now prove that $\frac{d}{d t}\left(\frac{R}{a^{\frac{\mu}{3}}}\right)$ is an absolute reciprocant of the same character as $\frac{R}{a^{\frac{\mu}{3}}}$. But $\frac{d}{d t}\left(\frac{R}{a^{\frac{\mu}{3}}}\right)=\frac{1}{a^{\frac{\mu}{3}}} \frac{d R}{d t}$. Hence $\frac{d R}{d t}$ is a reciprocant whose characteristic is $\mu$, and character the same as that of $R$.

The simplest Orthogonal Reciprocant is the form

$$
\left(1+t^{2}\right) b-3 a^{2} t,
$$

which occurs on p. 19 of Boole's Differential Equations. When equated to zero it is the general differential equation of a circle. It is noticeable that although Boole obtains this form by equating to zero the differential of the radius of curvature

$$
\frac{\left(1+t^{2}\right)^{\frac{3}{2}}}{a}
$$

he does not recognise the fact that it vanishes at points of maximum or minimum curvature of any plane curve, but says that the "geometrical property which this equation expresses is the invariability of the radius of curvature."

Taking this form as an example of our general theorem, let

$$
R=\left(1+t^{2}\right) b-3 a^{2} t
$$

then

$$
\frac{d R}{d t}=2 b t-3 a^{2}
$$


which is the familiar Schwarzian. Observe that

and

$$
\begin{aligned}
\left(1+t^{2}\right) b-3 a^{2} t & =-t^{6}\left\{\left(1+\tau^{2}\right) \beta-3 \alpha^{2} \tau\right\} \\
2 b t-3 a^{2} & =-t^{6}\left(2 \beta \tau-3 \alpha^{2}\right),
\end{aligned}
$$

so that the characteristic and character are the same for both these forms.

The form $c t-5 a b$, which we have called the Post-Schwarzian, when multiplied by 2 and integrated with respect to $t$, gives

$$
c t^{2}-10 a b t+\phi(a, b, \ldots) \text {. }
$$

In order that this may be a reciprocant, we must have

$$
\phi(a, b, \ldots)=c+15 a^{3}
$$

In this way the Orthogonal Reciprocant

was obtained originally.

$$
\left(1+t^{2}\right) c-10 a b t+15 a^{3}
$$

It will be easy to verify that this is a reciprocant by means of the identical relations

$$
\begin{aligned}
& t=\frac{1}{\tau} \\
& a=-\frac{\alpha}{\tau^{3}} \\
& b=-\frac{\beta \tau-3 \alpha^{2}}{\tau^{5}} \\
& c=-\frac{\gamma \tau^{2}-10 \alpha \beta \tau+15 \alpha^{3}}{\tau^{7}}
\end{aligned}
$$

We shall find that

$$
\left(1+t^{2}\right) c-10 a b t+15 a^{3}=-t^{7}\left\{\left(1+\tau^{2}\right) \gamma-10 \alpha \beta \tau+15 \alpha^{3}\right\}
$$

and comparing this with

$$
c t-5 a b=-\bar{t}(\gamma \tau-5 \alpha \beta)
$$

it will be noticed that both forms have the same character and the same characteristic.

The complete primitive of the differential equation

$$
c\left(1+t^{2}\right)-10 a b t+15 a^{3}=0
$$

has been found by Mr Hammond and Prof. Greenhill. The solution may be written in the following forms :

$$
\begin{aligned}
& x=\int \frac{d t}{\sqrt{\left\{\kappa\left(1-15 t^{2}+15 t^{4}-t^{6}\right)+\lambda\left(6 t-20 t^{3}+6 t^{5}\right)\right\}}}+\mu \\
& \left.y=\int \frac{t d t}{\sqrt{\left\{\kappa\left(1-15 t^{2}+15 t^{4}-t^{6}\right)+\lambda\left(6 t-20 t^{3}+6 t^{5}\right)\right\}}+\nu}\right\} \\
& x=\int \frac{\cos (\theta-A) d \theta}{\sqrt{ }\{B \cos 6(\theta-A)\}}+\text { const. } \\
& \left.y=\int \frac{\sin (\theta-A) d \theta}{\sqrt{ }\{B \cos 6(\theta-A)\}}+\text { const. }\right\} \text {. }
\end{aligned}
$$


where

$$
k^{\prime 2} n^{2}(X, k)=k^{2} t n^{2}\left(Y, k^{\prime}\right)
$$

and

$$
k=\sin 15^{\circ}, \quad k^{\prime}=\sin 75^{\circ},
$$

$$
\begin{gathered}
X=l x+m y+n_{1}, \\
Y=m x-l y+n_{2},
\end{gathered}
$$

$l, m, n_{1}, n_{2}$ being arbitrary constants.

The last two forms of solution are due to Prof. Greenhill.

\section{LECTURE VII.}

I have frequently referred to, and occasionally dilated on, the analogy between pure reciprocants and invariants. A new bond of connection between the two theories has been established by Capt. MacMahon, which I will now explain. Let me, by way of preface, so far anticipate what I shall have to say on the Theorem of Aggregation in Invariants (that is, the theorem concerning the number of linearly independent invariants of a given type) as to remark that the proof of this theorem, first given by me in Crelle's Journal and subsequently in the Phil. Mag. for March, 1878, depends on the fact that if we take two operators, namely, the Annihilator, say

and its opposite, say

$$
\Omega=a_{0} \partial_{a_{1}}+2 a_{1} \partial_{a_{2}}+3 a_{2} \partial_{a_{3}}+\ldots+j a_{j-1} \partial_{a_{j}}
$$

$$
O=a_{j} \partial_{a_{j-1}}+2 a_{j-1} \partial_{a_{j-2}}+3 a_{j-2} \partial_{a_{j-3}}+\ldots+j a_{1} \partial_{a_{0}}
$$

then $(\Omega O-O \Omega) I$ is a multiple of $I$.

Thus, if $I$ stands for any invariant (that is, if $\Omega I=0$ ), it follows immediately that $\Omega O I$ is a multiple of $I$, and consequently $\Omega^{m} O^{m} I$ is also a multiple of $I$. We may call $\Omega$ and $O$, which are exact opposites to each other, reversing operators.

Now, MacMahon has found out the reversor to $V$, the Annihilator of pure reciprocants. His reversing operator is no longer of a similar, though opposite, form to $V$, as $O$ is to $\Omega$, but is simply $\frac{d}{d x}$; nor is the effect of operating with $V \frac{d}{d x}$ on any pure reciprocant $R$ equivalent to multiplication by a merely numerical factor, as was the case with $\Omega O I$, but $\left(V \frac{d}{d x}\right) R$ is a numerical multiple of $a R$, and as a consequence of this $\left(V^{m} \frac{d^{m}}{d x^{m}}\right) R$ is a numerical multiple of $a^{m} R$. Thus the parallelism is like that between the two sexes, the same with a difference, as is usually the case in comparing the two theories. 
This remarkable relation between the operators $V$ and $\frac{d}{d x}$ may be seen a priori if we assume that (as we shall hereafter prove) to each pure reciprocant $R$ there is an annihilator $V$ of the form

$$
3 a^{2} \partial_{b}+(\ldots) \partial_{c}+(\ldots) \partial_{d}+(\ldots) \partial_{e}+\ldots
$$

not containing $\partial_{a}$ and linear in the remaining differential operators $\partial_{b}, \partial_{c}, \partial_{d}, \ldots$ For if we call the characteristic $\mu$, by differentiating the absolute pure reciprocant $\frac{R}{a^{\frac{\mu}{3}}}$ with respect to $x$ we obtain, as was shown in the last lecture, the pure reciprocant

$$
3 a \frac{d R}{d x}-\mu b R
$$

Since this is annihilated by $V$, we have

$$
3 a\left(V \frac{d}{d x}\right) R-\mu R V b-\mu b V R=0 .
$$

But, since $R$ is a pure reciprocant, $V R=0$; and from the assumed form of $V$ it follows that

$$
\begin{array}{cc}
V b=3 a^{2} . \\
\text { or } \quad 3 a\left(V \frac{d}{d x}\right) R-3 \mu a^{2} R=0, \\
\left(V \frac{d}{d x}\right) R=\mu a R .
\end{array}
$$

Thus the operation of $V \frac{d}{d x}$ is equivalent to multiplication by $\mu a$, so that (barring the introduction of a) $V$ restores to $\frac{d R}{d x}$ the form it had antecedent to the operation of $\frac{d}{d x}$, and may be called a qualified reversor to $\frac{d}{d x}$.

For example, suppose that

$$
R=3 a c-5 b^{2}
$$

Since we are using natural letters for the derivatives of $y$ with respect to $x$, we have

$$
\frac{d}{d x}=b \partial_{a}+c \partial_{b}+d \partial_{c}+\ldots
$$

and, as we shall presently see,

$$
\begin{aligned}
& \qquad V=3 a^{2} \partial_{b}+10 a b \partial_{c}+\left(15 a c+10 b^{2}\right) \partial_{d}+\ldots \\
& \text { Now, } \frac{d R}{d x}=\left(b \partial_{a}+c \partial_{b}+d \partial_{c}\right)\left(3 a c-5 b^{2}\right)=3 b c-10 b c+3 a d=3 a d-7 b c \\
& \text { Operating on this with } V \text {, we find } \\
& V \frac{d R}{d x}=V(3 a d-7 b c)=-21 a^{2} c-70 a b^{2}+3 a\left(15 a c+10 b^{2}\right)=24 a^{2} c-40 a b^{2} \\
& \text { that is } \quad V \frac{d}{d x}\left(3 a c-5 b^{2}\right)=8 a\left(3 a c-5 b^{2}\right)
\end{aligned}
$$


Let us now inquire whether it is possible so to determine an operator $V$ that the relation

$$
\left(V \frac{d}{d x}-\frac{d}{d x} V\right) F=(3 i+w) a F
$$

may be satisfied identically when $F$ is any homogeneous isobaric function of the letters $a, b, c, \ldots$ of degree $i$ and weight $w$. If so, we must be able to satisfy each of the equations

$$
\begin{aligned}
& \left(V \frac{d}{d x}-\frac{d}{d x} V\right) a=3 a^{2} \\
& \left(V \frac{d}{d x}-\frac{d}{d x} V\right) b=4 a b \\
& \left(V \frac{d}{d x}-\frac{d}{d x} V\right) c=5 a c \\
& \left(V \frac{d}{d x}-\frac{d}{d x} V\right) d=6 a d
\end{aligned}
$$

which are found by writing $a, b, c, d, \ldots$ successively in the place of $F$. written

Now $\frac{d a}{d x}=b, \frac{d b}{d x}=c, \frac{d c}{d x}=d, \ldots$ so that the above equations may be

$$
\begin{aligned}
& V b=3 a^{2}+\frac{d}{d x}(V a), \\
& V c=4 a b+\frac{d}{d x}(V b), \\
& V d=5 a c+\frac{d}{d x}(V c), \\
& V e=6 a d+\frac{d}{d x}(V d),
\end{aligned}
$$

These equations are sufficient to completely determine $V$ on the supposition previously made that it is linear in the differential operators and does not contain $\partial_{a}$; for, since $V$ is linear, it must be of the form

$$
(\nabla a) \partial_{a}+(V b) \partial_{b}+(V c) \partial_{c}+\ldots
$$

and, since it does not contain $\partial_{a}$, we must have $V a=0$, and therefore

$$
\begin{aligned}
& V b=3 a^{2}, \\
& V c=4 a b+\frac{d}{d x}\left(3 a^{2}\right)=4 a b+6 a b=10 a b, \\
& V d=5 a c+\frac{d}{d x}(10 a b)=5 a c+10 b^{2}+10 a c=15 a c+10 b^{2}, \\
& V e=6 a d+\frac{d}{d x}\left(15 a c+10 b^{2}\right)=6 a d+15 b c+20 b c+15 a d=21 a d+35 b c
\end{aligned}
$$

Hence $\quad \nabla=3 a^{2} \partial_{b}+10 a b \partial_{c}+\left(15 a c+10 b^{2}\right) \partial_{d}+(21 a d+35 b c) \partial_{e}+\ldots$ 
When the modified letters $a_{0}, a_{1}, a_{2}, \ldots$ are used, we shall have, in consequence of the change of notation, $\left(V \frac{d}{d x}\right) R=2 \mu a_{0} R$ (instead of $\mu a R$ ). If, as before, we seek to satisfy the equation

$$
\left(V \frac{d}{d x}-\frac{d}{d x} V\right) F=2(3 i+w) a_{0} F
$$

we shall find, on writing $a_{n}$ in the place of $F$,

$$
\left(V \frac{d}{d x}-\frac{d}{d x} V\right) a_{n}=2(3+n) a_{0} a_{n}
$$

This condition will be sufficient, as well as necessary, for the satisfaction of (1) when $V$ is linear; for then

$$
V \frac{d}{d x}-\frac{d}{d x} V
$$

will also be linear, its general term being

$$
\left(V \frac{d a_{n}}{d x}-\frac{d}{d x} V a_{n}\right) \partial_{a_{n}}
$$

which is equal to $2(3+n) a_{0} a_{n} \partial_{a_{n}}$ by equation (2). Hence

$$
\begin{aligned}
\left(V \frac{d}{d x}-\frac{d}{d x} V\right) F & =\text { a sum of terms of the form } 2(3+n) a_{0} a_{n} \partial_{a_{n}} F \\
& =2 a_{0}\left(3 a_{0} \partial_{a_{0}}+3 a_{1} \partial_{a_{1}}+3 a_{2} \partial_{a_{2}}+\ldots\right) F \\
& +2 a_{0}\left(a_{1} \partial_{a_{1}}+2 a_{2} \partial_{a_{2}}+\ldots\right) F
\end{aligned}
$$

that is, equation (1) is satisfied whenever (2) is. Writing in (2)

$$
\frac{d a_{n}}{d x}=(n+3) a_{n+1}
$$

we obtain

$$
(n+3) V a_{n+1}=2(n+3) a_{0} a_{n}+\frac{d}{d x}\left(V a_{n}\right),
$$

from which the values of $V a_{n}$ may be successively determined.

When $V a_{0}=0$, the value of $V a_{n}$, which satisfies (3), is

$$
V a_{n}=\frac{n+3}{2}\left(a_{0} a_{n-1}+a_{1} a_{n-2}+\ldots+a_{n-2} a_{1}+a_{n-1} a_{0}\right) ;
$$

thus

$$
V a_{1}=\frac{4}{2} \cdot a_{0}^{2}, V a_{2}=5 a_{0} a_{1}, V a_{3}=6 a_{0} a_{2}+3 a_{1}^{2}, \ldots
$$

and the value of $V$ is therefore

$$
\frac{4}{2} \cdot a_{0}^{2} \partial_{a_{1}}+5 a_{0} a_{1} \partial_{a_{2}}+6\left(a_{0} a_{2}+\frac{1}{2} a_{1}^{2}\right) \partial_{a_{3}}+7\left(a_{0} a_{3}+a_{1} a_{2}\right) \partial_{a_{4}}+\ldots
$$

Now that we are on the subject of parallelism between the old and new worlds of Algebraical Form, I feel tempted to point out yet another very interesting bond of connection between them. There is a theorem concerning Invariants which I am not aware that any one but myself has noticed, or at 
all events I do not remember ever seeing it in print*, which is this: If we take any "invariant" and regard its most advanced letter as a variable, or say rather as the ratio of two variables $u: v$, by multiplying by a proper power of $v$ we obtain a new Quantic in $u$, $v$; or, if we take any number of such invariants with the same most advanced letter (or, as we may call it in a double sense, the same radical letter) in common, we shall have a system of binary Quantics in $u, v$. My theorem is, or was, that an Invariant of any one or more of such Quantics is an Invariant of the original Quantic. I recently found a similar proposition to be true for Reciprocants, namely, forming as before a system of pure Reciprocants into Quantics in $u, v$, any "Invariant" of such system is itself a Reciprocant.

The two theorems may be stated symbolically thus:

$$
\left.\begin{array}{l}
I I^{\prime}=I^{\prime \prime} \\
I R=R^{\prime}
\end{array}\right\}
$$

On mentioning this to Mr L. J. Rogers, he sent me next day a proof which, although only stated as applicable to Reciprocants, is equally so, mutatis mutandis, to Invariants. Although given for a single invariant, it applies equally to a system.

I give Mr Rogers' proof that any invariant of a pure reciprocant (the proof will not hold for impure ones) is a pure reciprocant; or rather I use his method to prove the analogous theorem that any invariant of an invariant is itself an invariant. It will be seen hereafter that this same proof applies to pure reciprocants with only trifling changes; but the proof as given by Mr Rogers requires some further considerations to be gone into for which we are not yet ripe.

Consider, for the sake of simplicity, the binary Quintic

$$
(a, b, c, d, e, f \gamma x, y)^{5} \text {, }
$$

and let $I$ be any invariant of it (satisfied or unsatisfied); then

$$
I=a_{0} f^{n}+a_{1} f^{n-1}+a_{2} f^{n-2}+\ldots+a_{n},
$$

where $a_{0}, a_{1}, a_{2}, \ldots a_{n}$ do not contain $f$, but are functions of $a, b, c, d, e$ alone. then

Let the Protomorphs for our Quintic be denoted by $A, B, C, D, E, F$;

$$
F=a^{2} f-5 a b e+2 a c d+8 b^{2} d+6 b c^{2} .
$$

Eliminating $f$ from $I$ by means of this equation, we have

$$
I a^{2 n}=A_{0} F^{n}+A_{1} F^{n-1}+A_{2} F^{n-2}+\ldots+A_{n},
$$

where $A_{0}, A_{1}, A_{2}, \ldots A_{n}$ are all of them invariants (not necessarily integral

* The theorem is, however, given in Vol. xI. p. 98 of the Bulletin de la Sociêté Mathématique de France, in a paper by M. Perrin, which has only recently come under the lecturer's notice. 
forms, but this is immaterial to the proof, for $\Omega$, annihilates fractional and integral invariants alike). For

$$
\Omega\left(I a^{2 n}\right)=\Omega\left(A_{0} F^{n}+A_{1} F^{n-1}+\ldots+A_{n}\right)
$$

and, in consequence of $I a^{2 n}$ and $F$ being invariants, so that, as regards $\Omega$, $F$ may be treated as if it were a constant, this becomes

$$
0=F^{n} \Omega A_{0}+F^{n-1} \Omega A_{1}+F^{n-2} \Omega A_{2}+\ldots+\Omega A_{n},
$$

in which the coefficients of the several powers of $F$ must be separately equated to zero. In other words, $A_{0}, A_{1}, A_{2}, \ldots A_{n}$ are all of them invariants. Now, any invariant of

$$
A_{0} F^{n}+A_{1} F^{n-1}+A_{2} F^{n-2}+\ldots+A_{n}
$$

is a function of $A_{0}, A_{1}, A_{2}, \ldots A_{n}$, and therefore an invariant.

(N.B.-We cannot assume that any function of general reciprocants is itself a reciprocant.)

Again, since $\quad A_{0} F^{n}+\ldots+A_{n}$, and $a_{0} f^{n}+\ldots+a_{n}$ are connected by the substitution

$$
F=a^{2} f-5 a b e+\ldots,
$$

which is linear in respect to the letters $F$ and $f$, any invariant of

$$
A_{0} F^{n}+\ldots+A_{n}
$$

is (to a factor près, that factor being a power of $a$ which is itself an invariant) equal to the corresponding invariant of

$$
a_{0} f^{n}+\ldots+a_{n}
$$

But every invariant of the former has been shown to be an invariant of the original quantic, and therefore every invariant of the latter is so also.

I add some examples in illustration of this theorem :

Ex. 1. Take the invariant of the Quintic

$$
\begin{array}{r}
a^{2} f^{2}-10 a b e f+4 a c d f+16 b^{2} d f-12 b c^{2} f+16 a c e^{2}+9 b^{2} e^{2}-12 a d^{2} e-76 b c d e \\
+48 c^{3} e+48 b d^{3}-32 c^{2} d^{2}
\end{array}
$$

The discriminant of this, considered as a quadratic in $f$, is

$$
\begin{gathered}
a^{2}\left(16 a c e^{2}+9 b^{2} e^{2}-12 a d^{2} e-76 b c d e+48 c^{3} e+48 b d^{3}-32 c^{2} d^{2}\right) \\
-\left(5 a b e-2 a c d-8 b^{2} d+6 b c^{2}\right)^{2} \\
=16 a^{3} c e^{2}-16 a^{2} b^{2} e^{2}-12 a^{3} d^{2} e-56 a^{2} b c d e+48 a^{2} c^{3} e+80 a b^{3} d e-60 a b^{2} c^{2} e \\
+48 a^{2} b d^{3}-36 a^{2} c^{2} d^{2}-32 a b^{2} c d^{2}-64 b^{4} d^{2}+24 a b c^{3} d+96 b^{3} c^{2} d-36 b^{2} c^{4} .
\end{gathered}
$$

It will be found on trial that this is divisible by the invariant

the quotient being

$$
4\left(a e-4 b d+3 c^{2}\right)
$$

$$
\begin{aligned}
& 4 a^{2} c e-4 a b^{2} e-3 a^{2} d^{2}+2 a b c d+4 b^{3} d-3 b^{2} c^{2} \\
& =3 a\left(a c e-b^{2} e-a d^{2}+2 b c d-c^{3}\right)+\left(a c-b^{2}\right)\left(a e-4 b d+3 c^{2}\right)
\end{aligned}
$$


Thus the discriminant of the quadratic in $f$, that is, of the invariant

$$
a^{2} f^{2}-2 f\left(5 a b e-2 a c d+8 b^{2} d-6 b c^{2}\right)+\ldots
$$

is shown to be an invariant. It will further illustrate the proof of the theorem if we remark that precisely the same invariant is obtained by eliminating $f$ between the above form and the protomorph

$$
a^{2} f-5 a b e+2 a c d+8 b^{2} d-6 b c^{2} .
$$

Ex. 2. If we take the pure reciprocant

$$
45 a^{3} d^{2}-450 a^{2} b c d+400 a b^{3} d+192 a^{2} c^{3}+165 a b^{2} c^{2}-400 b^{4} c,
$$

which, from its similarity to the Discriminant of the Cubic, I have called the Quasi-Discriminant, and form its discriminant, when regarded as a quadratic in $d$, we find

$$
45 a^{3}\left(192 a^{2} c^{3}+165 a b^{2} c^{2}-400 b^{4} c\right)-\left(225 a^{2} b c-200 a b^{3}\right)^{2} .
$$

If, in this expression, we write $P=3 a c-5 b^{2}$, so that $3 a c=P+5 b^{2}$, it becomes

$$
\begin{aligned}
5.64 a^{2}\left(P+5 b^{2}\right)^{3}+5.165 a^{2} b^{2}\left(P+5 b^{2}\right)^{2}-15 . & 400 a^{2} b^{4}\left(P+5 b^{2}\right) \\
& -625 a^{2} b^{2}\left(3 P+7 b^{2}\right)^{2} .
\end{aligned}
$$

On performing the calculation it will be found that all the terms involving $b$ will disappear from this result, and there will remain the single term $320 a^{2} P^{3}$, that is, $320 a^{2}\left(3 a c-5 b^{2}\right)^{3}$, which is a reciprocant.

\section{LECTURE VIII.}

In my last lecture the complete expression, both in terms of the modified and unmodified letters, was obtained for $V$, the annihilator for pure reciprocants assuming its existence and its form. These assumptions I shall now make good by proving, from first principles, the fundamental theorem that the satisfaction of the equation

$$
V R=0
$$

is a necessary and sufficient condition in order that $R$ may be a pure reciprocant.

It will be advantageous to use the modified system of letters, in which

$$
t, a_{0}, a_{1}, a_{2}, \ldots \text { stand for } \frac{d y}{d x}, \frac{1}{1.2} \cdot \frac{d^{2} y}{d x^{2}}, \frac{1}{1.2 .3} \cdot \frac{d^{3} y}{d x^{3}}, \frac{1}{1.2 .3 .4} \cdot \frac{d^{4} y}{d x^{4}}, \ldots
$$

and $\quad \alpha_{0}, \alpha_{1}, \alpha_{2}, \ldots$ for $\frac{1}{1.2} \cdot \frac{d^{2} x}{d y^{2}}, \frac{1}{1.2 .3} \cdot \frac{d^{3} x}{d y^{3}}, \frac{1}{1.2 .3 .4} \cdot \frac{d^{4} x}{d y^{4}}, \ldots$

respectively. Let the variation due to the change of $x$ into $x+\epsilon y$, where $\epsilon$ 
is an infinitesimal number, be denoted by $\Delta$. Obviously this change leaves the value of each of the quantities $\alpha_{0}, \alpha_{1}, \alpha_{2}, \ldots$ unaltered, and therefore

$$
\Delta R\left(\alpha_{0}, \alpha_{1}, \alpha_{2}, \ldots\right)=0,
$$

whatever the nature of $R$ may be. But when $R$ is a pure reciprocant,

$$
R\left(a_{0}, a_{1}, a_{2}, \ldots\right)= \pm t^{\mu} R\left(\alpha_{0}, \alpha_{1}, \alpha_{2}, \ldots\right), \quad .
$$

whence it immediately follows that

$$
\Delta t^{-\mu} R\left(a_{0}, a_{1}, a_{2}, \ldots\right)=0 * \text {. }
$$

Before proceeding to determine the values of

$$
\Delta t, \Delta a_{0}, \Delta a_{1}, \Delta a_{2}, \ldots
$$

it will be useful to remark that since

$$
\frac{d y}{d x}=t, \frac{d^{2} y}{d x^{2}}=1.2 \cdot a_{0}, \frac{d^{3} y}{d x^{3}}=1.2 .3 \cdot a_{1}, \ldots
$$

we have

$$
\frac{d t}{d x}=2 a_{0}, \frac{d a_{0}}{d x}=3 a_{1}, \ldots,
$$

and generally $\frac{d a_{n}}{d x}=(n+3) a_{n+1}$.

Now let $[t]$ denote the augmented value of $t$, and in general let [ ] be used to signify that the augmented value of the quantity enclosed in it is to be taken. Then

$$
[t]=\frac{d y}{d[x]}=\frac{d y}{d(x+\epsilon y)}=\frac{d y}{d x\left(1+\epsilon \frac{d y}{d x}\right)}=\frac{t}{1+\epsilon t}=t-\epsilon t^{2}
$$

so also $2\left[a_{0}\right]=\left[2 a_{0}\right]=\frac{d[t]}{d[x]}=\frac{d[t]}{d(x+\epsilon y)}=\frac{d[t]}{d x(1+\epsilon t)}=(1-\epsilon t) \frac{d[t]}{d x}$

$$
\begin{aligned}
& =(1-\epsilon t) \frac{d}{d x}\left(t-\epsilon t^{2}\right)=(1-\epsilon t)\left(2 a_{0}-4 \epsilon t a_{0}\right) \\
& =2 a_{0}-6 \epsilon t a_{0}
\end{aligned}
$$

that is

$$
\left[a_{0}\right]=a_{0}-3 \epsilon t a_{0} \text {. }
$$

Reasoning precisely similar to that which gave

$$
2\left[a_{\theta}\right]=(1-\epsilon t) \frac{d}{d x}[t]
$$

leads to the formula

$$
(n+3)\left[a_{n+1}\right]=(1-\epsilon t) \frac{d}{d x}\left[a_{n}\right]
$$

* It has been"suggested by Mr J. Chevallier that the proof might be simplified by considering the variation $\Delta a_{0}{ }^{-\frac{\mu}{3}} R_{j}^{\top}\left(a_{0}, a_{1}, a_{2}, \ldots\right)$ instead of $\Delta t^{-\mu} R\left(a_{0}, a_{1}, a_{2}, \ldots\right)$. 
from which the augmented values of $a_{1}, a_{2}, a_{3}, \ldots$ may be found by giving to $n$ the values $0,1,2, \ldots$ in succession. Thus, writing $n=0$, we have

or

$$
\begin{aligned}
3\left[a_{1}\right] & =(1-\epsilon t) \frac{d}{d x}\left[a_{0}\right]=(1-\epsilon t) \frac{d}{d x}\left(a_{0}-3 \epsilon t a_{0}\right) \\
& =(1-\epsilon t)\left(3 a_{1}-9 \epsilon t a_{1}-6 \epsilon a_{0}^{2}\right)=3 a_{1}-\epsilon\left(12 t a_{1}+6 a_{0}^{2}\right), \\
{\left[a_{1}\right] } & =a_{1}-\epsilon\left(4 t a_{1}+2 a_{0}^{2}\right) .
\end{aligned}
$$

Similarly, when $n=1$,

$$
\begin{aligned}
4\left[a_{2}\right] & =(1-\epsilon t) \frac{d}{d x}\left[a_{1}\right]=(1-\epsilon t) \frac{d}{d x}\left(a_{1}-4 \epsilon t a_{1}-2 \epsilon a_{0}^{2}\right) \\
& =(1-\epsilon t)\left(4 a_{2}-16 \epsilon t a_{2}-20 \epsilon a_{0} a_{1}\right) \\
& =4 a_{2}-20 \epsilon t a_{2}-20 \epsilon a_{0} a_{1},
\end{aligned}
$$

and

$$
\left[a_{2}\right]=a_{2}-\check{\Sigma} \epsilon\left(t a_{2}+a_{0} a_{1}\right) \text {. }
$$

$$
\text { Again, } \begin{aligned}
5\left[a_{3}\right] & =(1-\epsilon t) \frac{d}{d x}\left[a_{2}\right]=(1-\epsilon t) \frac{d}{d x}\left(a_{2}-5 \epsilon t a_{2}-5 \epsilon a_{0} a_{1}\right) \\
& =(1-\epsilon t)\left(5 a_{3}-25 \epsilon t a_{3}-30 \epsilon a_{0} a_{2}-15 \epsilon a_{1}^{2}\right) \\
& =5 a_{3}-30 \epsilon t a_{3}-30 \epsilon a_{0} a_{2}-15 \epsilon a_{1}^{2},
\end{aligned}
$$

so that $\quad\left[a_{3}\right]=a_{3}-\epsilon\left(6 t a_{3}+6 a_{0} a_{2}+3 a_{1}^{2}\right)$.

In like manner we shall find

$$
\left[a_{4}\right]=a_{4}-7 \epsilon\left(t a_{4}+a_{0} a_{3}+a_{1} a_{2}\right) .
$$

These results may be written in a more symmetrical form; thus:

$$
\begin{aligned}
& 2[t]=2 t-2 \epsilon t^{2}, \\
& 2\left[a_{0}\right]=2 a_{0}-3 \epsilon\left(t a_{0}+a_{0} t\right), \\
& 2\left[a_{1}\right]=2 a_{1}-4 \epsilon\left(t a_{1}+a_{0}^{2}+a_{1} t\right), \\
& 2\left[a_{2}\right]=2 a_{2}-5 \epsilon\left(t a_{2}+a_{0} a_{1}+a_{1} a_{0}+a_{2} t\right), \\
& 2\left[a_{3}\right]=2 a_{3}-6 \epsilon\left(t a_{3}+a_{0} a_{2}+a_{1}^{2}+a_{2} a_{0}+a_{3} t\right), \\
& 2\left[a_{4}\right]=2 a_{4}-7 \epsilon\left(t a_{4}+a_{0} a_{3}+a_{1} a_{2}+a_{2} a_{1}+a_{3} a_{0}+a_{4} t\right) .
\end{aligned}
$$

The general law

$$
2\left[a_{n}\right]=2 a_{n}-(n+3) \epsilon\left(t a_{n}+a_{0} a_{n-1}+\ldots+a_{n-1} a_{0}+a_{n} t\right),
$$

or, as it may also be written,

$$
\Delta a_{n}=-\frac{n+3}{2} \epsilon\left(t a_{n}+a_{0} a_{n-1}+\ldots+a_{n-1} a_{0}+a_{n} t\right)
$$

admits of an easy inductive proof.

Assuming the truth of the theorem for $\left[a_{n}\right]$, and writing for brevity in what follows,

$$
S_{n}=t a_{n}+a_{0} a_{n-1}+a_{1} a_{n-2}+\ldots+a_{n-2} a_{1}+a_{n-1} a_{0}+a_{n} t,
$$

we have

$$
\left[a_{n}\right]=a_{n}-\frac{n+3}{2} \epsilon S_{n} .
$$




$$
\text { Now, } \quad \begin{aligned}
\frac{d S_{n}}{d x}=(n & +3) t a_{n+1}+2 a_{0} a_{n} \\
& +(n+2) a_{0} a_{n}+3 a_{1} a_{n-1} \\
& +(n+1) a_{1} a_{n-1}+4 a_{2} a_{n-2} \\
& +\ldots \ldots \ldots \ldots \ldots \ldots+\ldots \ldots \ldots . . \ldots \ldots \\
& +4 a_{n-2} a_{2}+(n+1) a_{n-1} a_{1} \\
& +3 a_{n-1} a_{1}+(n+2) a_{n} a_{0} \\
& +2 a_{n} a_{0}+(n+3) a_{n+1} t \\
=(n+4)\left(t a_{n+1}+a_{0} a_{n}+a_{1} a_{n-1}+\ldots\right. & \left.\quad+a_{n-1} a_{1}+a_{n} a_{0}+a_{n+1} t\right)-2 t a_{n+1} \\
=(n & +4) S_{n+1}-2 t a_{n+1} .
\end{aligned}
$$

Hence $\frac{d}{d x}\left[a_{n}\right]=(n+3) a_{n+1}-\frac{n+3}{2} \epsilon\left\{(n+4) S_{n+1}-2 t a_{n+1}\right\}$.

But, as we have already seen,

consequently,

$$
(n+3)\left[a_{n+1}\right]=(1-\epsilon t) \frac{d}{d x}\left[a_{n}\right]
$$

$$
\left[a_{n+1}\right]=(1-\epsilon t) a_{n+1}-\frac{n+4}{2} \epsilon S_{n+1}+\epsilon t a_{n+1}=a_{n+1}-\frac{n+4}{2} \epsilon S_{n+1} ;
$$

that is, the theorem holds for $\left[a_{n+1}\right]$ when it holds for $\left[a_{n}\right]$. But we know that it is true for the cases $n=0,1,2,3,4$, and therefore it is true universally.

Resuming the proof of the main theorem, it has been shown that

that is

$$
\Delta t^{-\mu} R\left(a_{0}, a_{1}, a_{2}, \ldots\right)=0 \text {; }
$$

or

$$
-\mu t^{-1} \Delta t+R^{-1} \Delta R=0
$$

$$
-\mu R t^{-1} \Delta t+\frac{d R}{d a_{0}} \Delta a_{0}+\frac{d R}{d a_{1}} \Delta a_{1}+\frac{d R}{d a_{2}} \Delta a_{2}+\ldots=0 .
$$

But

$$
\begin{aligned}
& \Delta t=-\epsilon t^{2}, \\
& \Delta a_{0}=-3 \epsilon t a_{0}, \\
& \Delta a_{1}=-\epsilon\left(4 t a_{1}+2 a_{0}^{2}\right), \\
& \Delta a_{2}=-\epsilon\left(5 t a_{2}+5 a_{0} a_{1}\right), \\
& \Delta a_{3}=-\epsilon\left(6 t a_{3}+6 a_{0} a_{2}+3 a_{1}^{2}\right), \\
& \Delta a_{4}=-\epsilon\left(7 t a_{4}+7 a_{0} a_{3}+7 a_{1} a_{2}\right),
\end{aligned}
$$

and consequently

$$
\begin{gathered}
t\left(\mu-3 a_{0} \partial_{a_{0}}-4 a_{1} \partial_{a_{1}}-5 a_{2} \partial_{a_{2}}-6 a_{3} \partial_{a_{3}}-7 a_{4} \partial_{a_{4}}-\ldots\right) R \\
-\left\{4\left(\frac{a_{0}^{2}}{2}\right) \partial_{a_{1}}+5\left(a_{0} a_{1}\right) \partial_{a_{2}}+6\left(a_{0} a_{2}+\frac{a_{1}^{2}}{2}\right) \partial_{a_{3}}\right. \\
\left.+7\left(a_{0} a_{3}+a_{1} a_{2}\right) \partial_{a_{4}}+\ldots\right\} R=0 .
\end{gathered}
$$


This is equivalent to the two conditions

$$
\left(3 a_{0} \partial_{a_{0}}+4 a_{1} \partial_{a_{1}}+5 a_{2} \partial_{a_{2}}+\ldots\right) R=\mu R
$$

and

$$
V R=0 \text {, }
$$

where

$$
V=4\left(\frac{a_{0}^{2}}{2}\right) \partial_{a_{1}}+5\left(a_{0} a_{1}\right) \partial_{a_{2}}+6\left(a_{0} a_{2}+\frac{a_{1}^{2}}{2}\right) \partial_{a_{3}}+7\left(a_{0} a_{3}+a_{1} a_{2}\right) \partial_{a_{4}}+\ldots .
$$

For greater simplicity I confine what I have to say to the only essential case, to which every other may be reduced, of a homogeneous pure reciprocant. The equation

$$
\left(3 a_{0} \partial_{a_{0}}+4 a_{1} \partial_{a_{1}}+5 a_{2} \partial_{a_{2}}+\ldots\right) R=\mu R
$$

shows that for every term $w+3 i$ is constant; that is, $w$ is constant and therefore the function $R$ is isobaric. This is also immediately deducible from the form of the relations between $a_{0}, a_{1}, a_{2}, \ldots ; \alpha_{0}, \alpha_{1}, \alpha_{2}, \ldots$, and, what is important to notice, for future purposes,

$$
F\left(a_{0}, a_{1}, a_{2}, \ldots\right)-t^{\mu} F\left(\alpha_{0}, \alpha_{1}, \alpha_{2}, \ldots\right),
$$

when $F$ is a homogeneous isobaric function, and $\mu=w+3 i$ is itself a homogeneous function of $\left(a_{0}, a_{1}, a_{2}, \ldots\right)$, whose degree is the same as that of $F$.

The only condition affecting $R$, a function of $a_{0}, a_{1}, a_{2}, \ldots$, supposed homogeneous and isobaric, is

$$
V R=0 .
$$

I shall now prove the converse, that if $R=F\left(a_{0}, a_{1}, a_{2}, \ldots\right)$ (being homogeneous and isobaric) has $V$ for its annihilator, then $R$ is a pure reciprocant. Let $D$ be the value of $F\left(a_{0}, a_{1}, a_{2}, \ldots\right)-t^{\mu} F\left(\alpha_{0}, \alpha_{1}, \alpha_{2}, \ldots\right)$ expressed as a function of $a_{0}, a_{1}, a_{2}, \ldots$ alone. Then $D$ will be a function of the same type as $F\left(a_{0}, a_{1}, a_{2}, \ldots\right)$.

Suppose that

$$
\Delta D=0 ;
$$

that is, that the variation of $D$ due to the change of $x$ into $x+\epsilon y$ vanishes in virtue of the equation $V R=0$.

Let $D$ become $D^{\prime}$ when $y$ receives an arbitrary variation $y+\eta u$, where $\eta$ is an infinitesimal constant and $u$ an arbitrary function of $x$; then the variation of $D^{\prime}$ will vanish when $x$ is changed into $x+\epsilon y+\epsilon \eta u$, and consequently when $x$ is changed into $x+\epsilon y$ the variation of $D^{\prime}$ will also vanish. Hence

$$
\Delta D^{\prime}=0 \text {, }
$$

and if we take the difference of the variations of $D$ and $D^{\prime}$, we shall find

$$
\Delta\left(u^{\prime \prime} \frac{d}{d a_{0}} D+u^{\prime \prime \prime} \frac{d}{d a_{1}} D+u^{\mathrm{Iv}} \frac{d}{d a_{2}} D+\ldots\right)=0 .
$$

Now, the arbitrary nature of the function $u$ shows that we must have

$$
\Delta \frac{d}{d a_{0}} D=0, \quad \Delta \frac{d}{d a_{1}} D=0, \quad \Delta \frac{d}{d a_{2}} D=0, \ldots
$$


and if we reason on $\frac{d}{d a_{0}} D, \frac{d}{d a_{1}} D, \ldots$ in the same way as we have on $D$, we see that the variation $\Delta$ of each of the second differential derivatives of $D$ will also vanish; and, pursuing the same argument further, it will be evident that the $\Delta$ of any derivative of $D$, of any order whatever, with respect to $a_{0}, a_{1}, a_{2}, \ldots$ will vanish. Hence

$$
D=0 \text {; }
$$

for if this is not so we may, supposing $D$ to be a function of degree $i$ in the letters $a_{0}, a_{1}, a_{2}, \ldots$, take the $\Delta$ of each of the differential derivatives of $D$ of the order $i-1$; each of these variations would vanish by what precedes; that is, the variation due to the change of $x$ into $x+\epsilon y$ of each of the letters $a_{0}, a_{1}, a_{2}, \ldots$ contained in $D$ would be identically zero, which is absurd. We see, therefore, that when $\Delta D=0$ (that is, when $R$ is annihilated by $V$ ), $D=0$, or

$$
F\left(a_{0}, a_{1}, a_{2}, \ldots\right)=t^{\mu} F\left(\alpha_{0}, \alpha_{1}, \alpha_{2}, \ldots\right),
$$

which proves the converse proposition.

It will not fail to be noticed how much language, and as a consequence algebraical thought (for words are the tools of thought), is facilitated by the use of the concept of annihilation in lieu of that of equality as expressed by a partial differential equation.

It is somewhat to the point that in the recent two grand determinations of the order of precedence among the so-called fixed stars relative to our planet, as approximately represented by the intensities of the light from them which reaches the eye, the one is directed by the principle of annihilation, the other by that of equality. Prof. Pritchard's method essentially consists in determining what relative thicknesses of an interposed glass screen, effected by means of a sliding wedge of glass, will serve to extinguish the light of a star; that employed by Prof. Pickering depends on finding what degree of rotation of an interposed prism of Iceland spar (a Nicol Prism) will serve to bring to an equality the ordinary image of one star with the extraordinary one of another. As these intensities depend on the squared sines and cosines of this angle of rotation measured from the position of non-visibility of one of them, it follows that the tangent squared of the twist measures the relative intensities by this method.

Hereafter it will be shown that if $F$ is a homogeneous isobaric function of whose weights are reckoned as

$$
y, y^{\prime}, y^{\prime \prime}, y^{\prime \prime \prime}, \ldots
$$

$$
-2,-1,0,1, \ldots
$$

then, when $x$ becomes $x+h y$, where $h$ is any constant quantity, $F$ becomes

$$
(1+h t)^{-\mu} e^{-\frac{h V_{1}}{1+h t}} F,
$$

where $\quad t=y^{\prime}, V_{1}=-t^{2} \partial_{t}+V$, and $\mu=3 i+w$,

$i$ being the degree and $w$ the weight of $F$. 
From this, by an obvious course of reasoning, could be deduced as a particular case the condition of $F\left(a_{0}, a_{1}, a_{2}, \ldots\right)$ remaining a factor of its altered self when any linear substitutions are impressed on $x$ and $y$; namely, the necessary and sufficient condition is that $F$ has $V$ for its annihilator.

\section{LECTURE IX:}

The prerogative of a Pure Reciprocant is that it continues a factor of its altered self when the variables $x$ and $y$ are subjected to any linear substitution. Its form, like that of any other reciprocant, is of course persistent when the variables are interchanged; that is, when in the general substitution, in which $y$ is changed into

$$
\begin{array}{rr}
f y+g x+h \\
\text { and } x \text { into } & f^{\prime} y+g^{\prime} x+h^{\prime},
\end{array}
$$

we give the particular values $h=0, h^{\prime}=0, f=0, g^{\prime}=0, f^{\prime}=1, g=1$, to the constants. Stated geometrically, the theorem is that the evanescence of any pure reciprocant $R$ indicates a property independent of transformation of axes in a plane. We suppose $R$ to be homogeneous and isobaric in $a, b, c, \ldots$ (If it were not, the theorem could not hold, for either the change of $y$ into $\kappa y$ or that of $x$ into $\lambda x$ would destroy the form.)

The persistence, under any linear substitution, of the form of pure reciprocants may be easily established as follows:

By a semi-substitution understand one where one of the variables remains unaltered. There are two such semi-substitutions, namely, where $x$ remains unaltered, and where $y$ does.

(1) Let $x$ remain unaltered and $y$ become $f y+g x+h$; then $a, b, c, \ldots$ become $f a, f b, f c, \ldots$ respectively ; and therefore

$$
R(a, b, c, \ldots) \text { becomes } f^{i} R(a, b, c, \ldots),
$$

where $i$ is the degree of $R$.

(2) Let $y$ remain unchanged and $x$ become $f^{\prime} y+g^{\prime} x+h^{\prime}$. Then, instead of $R$, I look to its equal

that is, to

$$
\begin{array}{lc} 
& q t^{\mu} R(\alpha, \beta, \gamma, \ldots)(q= \pm 1) ; \\
\text { that is, to } & q \tau^{-\mu} R(\alpha, \beta, \gamma, \ldots), \\
\text { which becomes } & q\left(f^{\prime}+g^{\prime} \tau\right)^{-\mu} g^{\prime} R(\alpha, \beta, \gamma, \ldots) .
\end{array}
$$

Since $R$ is a reciprocant, this is equal to

$$
\frac{\tau^{\mu}}{\left(f^{\prime}+g^{\prime} \tau\right)^{\mu}} g^{\prime i} R(a, b, c, \ldots),
$$

or, replacing $\tau$ by its equivalent $\frac{1}{t}$,

s. IV.

$$
\left(f^{\prime} t+g^{\prime}\right)^{-\mu} g^{\prime i} R(a, b, c, \ldots) .
$$


Thus we see that the proposition is true for a semi-substitution of either kind. Consider now the complete substitution made by changing $y$ into

and $x$ into

$$
\begin{gathered}
f y+g x+h \\
F y+G x+H .
\end{gathered}
$$

If $f=0$ and $G=0$, then $\frac{d^{2} y}{d x^{2}}, \frac{d^{3} y}{d x^{3}}, \ldots$ become $\frac{g}{F^{2}} \cdot \frac{d^{2} x}{d y^{2}}, \frac{g}{F^{3}} \cdot \frac{d^{3} x}{d y^{3}}, \ldots$; so that $R(a, b, c, \ldots)$ becomes $\frac{g^{i}}{F^{2 i+w}} \cdot R(\alpha, \beta, \gamma, \ldots)$; and since this is equal to

the proposition is true.

$$
\frac{g^{i}}{F^{2 i+w}} \cdot q t^{-\mu} R(a, b, c, \ldots)
$$

But if either of the two letters $f, G$ (say $f$ ) is not zero, we may combine two semi-substitutions so as to obtain the complete substitution, in which $y$ changes into

and $x$ changes into

$$
\begin{gathered}
f y+g x+h, \\
F y+G x+H .
\end{gathered}
$$

(1) Substitute $y_{1}(=f y+g x+h)$ for $y$, and $x_{1}(=x)$ for $x$.

(2) Then substitute $y_{2}\left(=y_{1}\right)$ for $y_{1}$, and $x_{2}\left(=f^{\prime} y_{1}+g^{\prime} x_{1}+h^{\prime}\right)$ for $x_{1}$.

By the first of these semi-substitutions

$$
R(a, b, c, \ldots)
$$

takes up an extraneous factor $f^{i}$. By the second it acquires the factor

$$
\left(f^{\prime} \frac{d y_{1}}{d x_{1}}+g^{\prime}\right)^{-\mu} g^{\prime i}, \text { where } \frac{d y_{1}}{d x_{1}}=f \frac{d y}{d x}+g=f t+g
$$

Hence we see that the extraneous factor is a negative power of a linear function of $t$, which we shall presently particularize, though it is not essential to the present demonstration to do so.

It only remains to show how the combination of these two semi-substitutions can be made to give the complete one in question. We have

and

$$
\begin{aligned}
y_{2} & =y_{1}=f y+g x+h \\
x_{2} & =f^{\prime} y_{1}+g^{\prime} x_{1}+h^{\prime}=f^{\prime}(f y+g x+h)+g^{\prime} x+h^{\prime} \\
& =f f^{\prime} y+\left(f^{\prime} g+g^{\prime}\right) x+\left(f^{\prime} h+h^{\prime}\right) .
\end{aligned}
$$

In order that this may be equal to $F y+G x+H$, we must be able to satisfy the equations

$$
f^{\prime}=\frac{F}{f}, \quad g^{\prime}=G-\frac{g F}{f}, \quad h^{\prime}=H-\frac{h F}{f},
$$

which is always possible, since by hypothesis $f$ is not żero. Similarly it may be shown that when $f$ vanishes, but $G$ does not, by substituting

(1) $x_{1}(=F y+G x+H)$ for $x$, and $y_{1}(=y)$ for $y$,

(2) $x_{2}\left(=x_{1}\right)$ for $x_{1}$, and $y_{2}\left(=f^{\prime \prime} y_{1}+g^{\prime \prime} x_{1}+h^{\prime \prime}\right)$ for $y_{1}$,

we may so determine $f^{\prime \prime}, g^{\prime \prime}, h^{\prime \prime}$ as to get the complete substitution as before. 
In every case, therefore, any linear substitution impressed upon the variables $x$ and $y$ will leave $R(a, b, c, \ldots)$ unaltered, barring the acquisition of an extraneous factor which is a negative power of a linear function of $t$.

Now, the first semi-substitution introduces, as we have seen, the constant factor

the second introduces the factor

$$
f^{i}
$$

$$
\left(f^{\prime} \frac{d y_{1}}{d x_{1}}+g^{\prime}\right)^{-\mu} g^{\prime i}
$$

where

$$
\frac{d y_{1}}{d x_{1}}=f t+g
$$

The complete extraneous factor is the product of these two, and is therefore

$$
f^{i} g^{\prime i}\left(f f^{\prime} t+f^{\prime} g+g^{\prime}\right)^{-\mu}
$$

To express $f^{\prime}$ and $g^{\prime}$ in terms of the constants of the complete substitution we have

$$
f^{\prime}=\frac{F}{f}, g^{\prime}=G-\frac{g F}{f}
$$

Writing these values for $f^{\prime}$ and $g^{\prime}$ in the expression just found, we obtain

$$
(f G-g F)^{i}(F t+G)^{-\mu}
$$

which is the extraneous factor acquired by $R$ when the complete substitution is made. For example, if $x$ becomes

and $y$ becomes

$$
\begin{aligned}
& F y+G x+H, \\
& f y+g x+h,
\end{aligned}
$$

the altered value of $a$ (that is, of $\left.\frac{d^{2} y}{d x^{2}}\right)$ is

$$
\left(f G-g F^{\prime}\right)(F t+G)^{-3} a \text {. }
$$

Corresponding to the simple interchange of the variables, we have

$$
F=1, G=0, H=0 ; f=0, g=1, h=0,
$$

so that

$$
f G-g F=-1
$$

and the altered value of $a$ is $\frac{d^{2} x}{d y^{2}}$, or

$$
\alpha=-\frac{a}{t^{3}}
$$

which is right. In this case the general value of the acquired extraneous factor

$$
\left(f G-g F^{\prime}\right)^{i}(F t+G)^{-\mu} \text { becomes }(-)^{i} t^{-\mu},
$$

thus showing, what we have already proved from other considerations, that the character of a pure reciprocant is odd or even according as its degree is odd or even. 
We saw in the last lecture that every pure reciprocant necessarily satisfied the two conditions

$$
\left(3 a_{0} \partial_{a_{0}}+4 a_{1} \partial_{a_{1}}+5 a_{2} \partial_{a_{2}}+\ldots\right) R=\mu R
$$

(where $\mu$ is the characteristic), and

$$
V R=0 .
$$

We also saw that $V R=0$ was a sufficient as well as necessary condition that any homogeneous function $R$ of $a_{0}, a_{1}, a_{2}, \ldots$ should be a pure reciprocant. It will now be shown that every pure reciprocant is either homogeneous and isobaric, or else resoluble into a sum of homogeneous and isobaric reciprocants. Non-homogeneous mixed ones, it may be observed, are not so resoluble, so that the theorem only holds for pure reciprocants.

(1) Let us suppose that $R$ (a pure reciprocant) is homogeneous in $a_{0}, a_{1}, a_{2} \ldots$; then it must be isobaric also. For, if $i$ is the degree of $R$, Euler's theorem shows that

$$
\left(3 a_{0} \partial_{a_{0}}+3 a_{1} \partial_{a_{1}}+3 a_{2} \partial_{a_{2}}+3 a_{3} \partial_{a_{3}}+\ldots\right) R=3 i R
$$

and since $R$ is a pure reciprocant, the condition

$$
\left(3 a_{0} \partial_{a_{0}}+4 a_{1} \partial_{a_{1}}+5 a_{2} \partial_{a_{2}}+6 a_{3} \partial_{a_{3}}+\ldots\right) R=\mu R
$$

is necessarily satisfied. Hence

$$
\left(a_{1} \partial_{a_{1}}+2 a_{2} \partial_{a_{2}}+3 a_{3} \partial_{a_{3}}+\ldots\right) R=(\mu-3 i) R=\text { a constant multiple of } R,
$$

which is the distinctive property of isobaric functions.

And, vice versâ, if $R$ is homogeneous and isobaric of weight $w$ and degree $i$, then

$$
\left(3 a_{0} \partial_{a_{0}}+4 a_{1} \partial_{a_{1}}+5 a_{2} \partial_{a_{2}}+\ldots\right) R=(w+3 i) R=\mu R .
$$

Thus homogeneous pure reciprocants are also isobaric and their characteristic is $3 i+w$. (This property is also true for mixed reciprocants, as we have previously shown.)

(2) Suppose that $R$ is not homogeneous, but made up of the homogeneous parts

$$
\text { Then, since } \quad V\left(R_{1}+R_{\prime \prime}+R_{\prime \prime}+\ldots\right)=0
$$

is satisfied identically, it is obvious that

$$
V R_{1}+V R_{1 \prime}+V R_{\text {II }}+\ldots=0
$$

must also be satisfied identically.

But since all the terms are of different degrees, the only way in which this can happen is by making $V R_{,}, V R_{\mu}, V R_{\mu \prime}, \ldots$ separately vanish. Now, $R_{1}, R_{1,}, R_{1,}, \ldots$ are by hypothesis homogeneous functions of $a_{0}, a_{1}, a_{2}, \ldots$, and it has just been shown that each of them is annihilated by $V$, which has been shown to be a sufficient condition that any homogeneous function of $a_{0}, a_{1}, a_{2}, \ldots$ may be a pure reciprocant. Thus each part $R_{,}, R_{\prime \prime}, R_{\prime \prime \prime}, \ldots$ of $R$ is a pure reciprocant. 
Also, the condition

$$
\left(3 a_{0} \partial_{a_{0}}+4 a_{1} \partial_{a_{1}}+5 a_{2} \partial_{a_{2}}+\ldots\right) R=\mu R
$$

shows that if $i_{1}, w_{1} ; i_{2}, w_{2} ; i_{3}, w_{3} ; \ldots$ are the deg. weights of $R_{1}, R_{\mu}, R_{\text {I, }}, \ldots$, we must have

$$
3 i_{1}+w_{1}=\mu, 3 i_{2}+w_{2}=\mu, 3 i_{3}+w_{3}=\mu, \ldots
$$

Thus non-homogeneous pure reciprocants are severable into parts each of which is a homogeneous and isobaric pure reciprocant, the characteristic of each part being equal to the same quantity $\mu$, which is the characteristic of the whole.

I will now explain what information concerning the number of pure reciprocants of a given type is afforded by the equation $V R=0$. Let

$$
A a_{0}^{\lambda_{0}} a_{1}^{\lambda_{1}} a_{2}^{\lambda_{2}} \ldots a_{j}^{\lambda_{j}}
$$

be a term of a homogeneous isobaric function (with its full number of terms) of $a_{0}, a_{1}, a_{2}, \ldots a_{j}$, whose degree is $i$, extent $j$, and weight $w$, and which we will call $R$.

Then in the entire function there are as many terms as there are solutions in integers of the equations

$$
\begin{aligned}
\lambda_{0}+\lambda_{1}+\lambda_{2}+\lambda_{3}+\ldots+\lambda_{j} & =i, \\
\lambda_{1}+2 \lambda_{2}+3 \lambda_{3}+\ldots+j \lambda_{j} & =w .
\end{aligned}
$$

In other words, the number of terms in $R$ is equal to the number of ways in which $w$ can be made up of $i$ or fewer parts, none greater than $j$. This number will be denoted by

$$
(w ; i, j)
$$

Since the function $R$ is the sum of every possible term of the form

$$
A a_{0}^{\lambda_{0}} a_{1}^{\lambda_{1}} \ldots a_{j}^{\lambda_{j}}
$$

each multiplied by an arbitrary constant, the number of these arbitrary constants is also

$$
(w ; i, j)
$$

Now, suppose $R$ to be a reciprocant; this imposes the condition

$$
V R=0 .
$$

Consider the effect produced by the operation of any term of

$$
V=4\left(\frac{a_{0}^{2}}{2}\right) \partial_{a_{1}}+5 a_{0} a_{1} \partial_{a_{2}}+6\left(a_{0} a_{2}+\frac{a_{1}^{2}}{2}\right) \partial_{a_{3}}+\ldots
$$

say $\left(a_{0} a_{2}+\frac{a_{1}^{2}}{2}\right) \partial_{a_{3}}$ (rejecting the numerical coefficient 6 ).

Operating on $R$ with $\partial_{a_{3}}$ decreases its weight by 3 and its degree by 1 unit. The subsequent multiplication by $a_{0} a_{2}+\frac{a_{1}^{2}}{2}$, on the other hand, increases the weight by 2 and the degree by 2 units. Hence the total effect 
of $\left(a_{0} a_{2}+\frac{a_{1}^{2}}{2}\right) \partial_{a_{3}}$ is to increase the degree by 1 and to diminish the weight by 1 unit. The same is evidently true for any other term of $V$. Thus the total effect of $V$ operating on the general homogeneous isobaric function $R$ of weight $w$, degree $i$, extent $j$, is to change it into another homogeneous isobaric function whose weight, degree and extent are respectively $w-1$, $i+1, j$. Observe that the extent is not altered by the operation of $V$.

It is easily seen that the coefficients of $V R$ are linear functions of the coefficients of $R$; for example, if

$$
\begin{aligned}
R & =A a_{0}{ }^{2} a_{3}+B a_{0} a_{1} a_{2}+C a_{1}^{3}, \\
V R & =a_{0}{ }^{3} a_{2}(6 A+2 B)+a_{0}{ }^{2} a_{1}{ }^{2}(3 A+5 B+6 C) .
\end{aligned}
$$

Hence the condition $V R=0$ gives us $(w-1 ; i+1, j)$ linear equations between the $(w ; i, j)$ coefficients of $R$; so that, assuming that these equations of condition are all independent, after they have been satisfied the number of arbitrary constants remaining in $R$ (that is, the number of linearly independent reciprocants of the type $w ; i, j)$ is equal to

$$
(w ; i, j)-(w-1 ; i+1, j)
$$

when this difference is positive; but when it is zero or negative there are no reciprocants of the given type.

If, however, any $r$ of the $(w-1 ; i+1, j)$ equations of condition should not be independent of the rest, these equations would be equivalent to $(w-1 ; i+1, j)-r$ independent conditions, and therefore the number of linearly independent reciprocants of the type $w ; i, j$ would be

$$
(w ; i, j)-(w-\mathbf{1} ; i+1, j)+r .
$$

It is therefore certain that this number cannot be less than

$$
(w ; i, j)-(w-1 ; i+1, j) \text {. }
$$

We shall assume provisionally that $r=0$, or in other words that the above partition formula is exact, instead of merely giving an inferior limit. Though it would be unsafe to rely on its accuracy, no positive grounds for doubting its exactitude have been revealed by calculation.

Such attempts as I have hitherto made to demonstrate the theorem have proved infructuous, but it must be remembered that more than a quarter of a century elapsed between the promulgation of Cayley's analogous theorem and its final establishment by myself on a secure basis of demonstration. 


\section{LECTURE $\mathrm{X}$.}

I will commence this lecture with a proof of Capt. MacMahon's theorem that if $R$ is any pure reciprocant and $\mu$ its characteristic (that is, its weight added to three times its degree),

$$
\left(V^{m} \frac{d^{m}}{d x^{m}}\right) R=1.2 .3 \ldots m\{\mu(\mu+2)(\mu+4) \ldots(\mu+2 m-2)\}\left(y^{\prime \prime}\right)^{m} R,
$$

where $y^{\prime \prime}$ may be replaced by either $2 a_{0}$ or $a$, according as the modified or unmodified system of letters is employed.

Instead of a pure reciprocant, let us consider any homogeneous isobaric function $F$ of degree $i$ and weight $w$; and (for the sake of simplicity writing $\partial_{x}$ for $\left.\frac{d}{d x}\right)$ instead of the operator $V^{m} \partial_{x}{ }^{m}$ let us consider $V^{m} \partial_{x}{ }^{n}-\partial_{x}{ }^{n} V^{m}$. We have identically

$$
\begin{aligned}
& \left(V^{m} \partial_{x}{ }^{n}-\partial_{x}{ }^{n} V^{m}\right) F=V^{m-1}\left(V \partial_{x}-\partial_{x} V\right) \partial_{x}{ }^{n-1} F \\
& +V^{m-2}\left(V \partial_{x}-\partial_{x} V\right) V \partial_{x}{ }^{n-1} F \\
& +V^{m-3}\left(V \partial_{x}-\partial_{x} V\right) V^{2} \partial_{x}^{n-1} F
\end{aligned}
$$

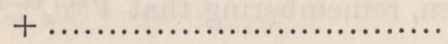

$$
\begin{aligned}
& +V\left(V \partial_{x}-\partial_{x} V\right) V^{m-2} \partial_{x}{ }^{n-1} F \\
& +\left(V \partial_{x}-\partial_{x} V\right) V^{m-1} \partial_{x}{ }^{n-1} F \\
& +\partial_{x}\left(V^{m} \partial_{x}^{n-1}-\partial_{x}^{n-1} V^{m}\right) F \text {. }
\end{aligned}
$$

Now, the operation of $\left(V \partial_{x}-\partial_{x} V\right)$ on any homogeneous isobaric function whose characteristic is $\mu_{1}$ is equivalent, as we have seen in Lecture VII, to multiplication by $\mu_{1} y^{\prime \prime}$; so that if the characteristics of

$$
\partial_{x}{ }^{n-1} F, \quad V \partial_{x}{ }^{n-1} F, \quad V^{2} \partial_{x}{ }^{n-1} F, \ldots V^{m-1} \partial_{x}{ }^{n-1} F
$$

are

$$
\mu_{1}, \mu_{2}, \mu_{3}, \ldots, \mu_{m},
$$

it follows that

$$
\begin{aligned}
\left(V^{m} \partial_{x}{ }^{n}-\partial_{x}{ }^{n} V^{m}\right)=\left(\mu_{1}+\mu_{2}+\mu_{3}+\ldots+\mu_{m}\right) y^{\prime \prime} V^{m-1} \partial_{x}{ }^{n-1} \boldsymbol{F} \\
\\
+\partial_{x}\left(V^{m} \partial_{x}{ }^{n-1}-\partial_{x}{ }^{n-1} V^{m}\right) F .
\end{aligned}
$$

Observe that

$$
V^{m-1}\left(V \partial_{x}-\partial_{x} V\right) \partial_{x}^{n-1} F=V^{m-1} \mu_{1} y^{\prime \prime} \partial_{x}{ }^{n-1} F=\mu_{1} y^{\prime \prime} V^{m-1} \partial_{x}{ }^{n-1} F,
$$

where the transposition of the $y^{\prime \prime}$ is permissible because $V$ does not act on it ; but if $y^{\prime \prime}$ were preceded by $\partial_{x}$ it could not be similarly transposed.

The numbers $\mu_{1}, \mu_{2}, \mu_{3}, \ldots$ form an arithmetical progression, for each operation of $V$ increases the degree by unity and diminishes the weight by unity, so that

$$
\begin{array}{cc}
\mu_{1}=3 i_{1}+w_{1} \text { becomes } \mu_{2}=3\left(i_{1}+1\right)+\left(w_{1}-1\right)=\mu_{1}+2 . \\
\text { Similarly } \quad \mu_{3}=\mu_{1}+4, \quad \mu_{4}=\mu_{1}+6, \ldots \mu_{m}=\mu_{1}+2 m-2 .
\end{array}
$$


The characteristic of $F$ being

$$
\mu=3 i+w, \text { that of } \partial_{x}^{n-1} F \text { is } \mu_{1}=\mu+n-1 ;
$$

for each operation of $\partial_{x}$ leaves the degree unaltered, but adds an unit to the weight; hence

so that

$$
\mu_{1}+\mu_{2}+\mu_{3}+\ldots+\mu_{m}=m(\mu+m+n-2)
$$

$\left(V^{m} \partial_{x}{ }^{n}-\partial_{x}{ }^{n} V^{m}\right) F=m(\mu+m+n-2) y^{\prime \prime} V^{m-1} \partial_{x}{ }^{n-1} F+\partial_{x}\left(V^{m} \partial_{x}{ }^{n-1}-\partial_{x}{ }^{n-1} V^{m}\right) F$.

When $F^{\prime}=R$, a pure reciprocant, so that $V R=0$, our formula becomes

$$
V^{m} \partial_{x}{ }^{n} R=m(\mu+m+n-2) y^{\prime \prime} V^{m-1} \partial_{x}{ }^{n-1} R+\partial_{x} V^{m} \partial_{x}{ }^{n-1} R \text {. }
$$

Suppose that in (2) $m>n$, then $V^{m} \partial_{x}^{n} R=0$. This is obviously true when $n=0$, and when $n=1$. When $n=2$ we find

$$
\begin{aligned}
V^{m} \partial_{x}{ }^{2} R & =m(\mu+m) y^{\prime \prime} V^{m-1} \partial_{x} R+\partial_{x} V^{m} \partial_{x} R \\
& =0 \text { if } m>2 .
\end{aligned}
$$

Similarly the case $n=3, m>3$ can be made to depend on $n=2, m>2$, and in general each case depends on the one immediately preceding it. Next let $n=m$ in (2); then, remembering that $V^{m} \partial_{x}{ }^{m-1} R=0$, we have

$$
V^{m} \partial_{x}{ }^{m} R=m(\mu+2 m-2) y^{\prime \prime} V^{m-1} \partial_{x}{ }^{m-1} R,
$$

from which MacMahon's theorem that

$$
V^{m} \partial_{x}{ }^{m} R=1.2 .3 \ldots m\{\mu(\mu+2)(\mu+4) \ldots(\mu+2 m-2)\}\left(y^{\prime \prime}\right)^{m} R
$$

is an immediate consequence.

Another special case of Formula (1) is worthy of notice, namely, that in which we take $n=1$, when we obtain the simple formula

$$
\left(V^{m} \partial_{x}-\partial_{x} V^{m}\right) F=m(\mu+m-1) y^{\prime \prime} V^{m-1} F \text {. }
$$

If in this we write $a_{n}$ in the place of $F$, and (the modified system of letters being used) $2 a_{0}$ for $y^{\prime \prime}, \mu$ becomes $3+n$, and we have

$$
\left(V^{m} \partial_{x}-\partial_{x} V^{m}\right) a_{n}=2 m(m+n+2) a_{0} V^{m-1} a_{n},
$$

or, as it may also be written,

$$
\frac{V^{m} \partial_{x} a_{n}}{1.2 .3 \ldots m}=\frac{\partial_{x} V^{m} a_{n}}{1.2 .3 \ldots m}+\frac{2(m+n+2) a_{0} V^{m-1} a_{n}}{1.2 .3 \ldots(m-1)}
$$

Mr Hammond remarks that this last formula may be used to prove the theorem

$$
\alpha_{n}=-t^{-n-3}\left(e^{-\frac{V}{t}}\right) a_{n},
$$

which was given without proof in Lecture II. Assuming that

$$
\alpha_{n}=-t^{-n-3} a_{n}+t^{-n-4} V a_{n}-t^{-n-5} \frac{V^{2} a_{n}}{1.2}+\ldots
$$


we have to prove that the theorem is also true when $n$ is increased by unity. Differentiating both sides of the assumed identity with respect to $x$, we find

$$
\begin{aligned}
\partial_{x} \alpha_{n}= & \partial_{x}\left(-t^{-n-3} a_{n}+t^{-n-4} V a_{n}-t^{-n-5} \frac{V^{2} a_{n}}{1.2}+\ldots\right) \\
= & -t^{-n-3} \partial_{x} a_{n}+t^{-n-4}\left\{\partial_{x} V a_{n}+2(n+3) a_{0} a_{n}\right\} \\
& -t^{-n-5}\left\{\frac{\partial_{x} V^{2} a_{n}}{1.2}+2(n+4) a_{0} V a_{n}\right\}
\end{aligned}
$$

the general term being

$$
(-)^{m+1} t^{-n-m-3}\left\{\frac{\partial_{x} V^{m} a_{n}}{1.2 .3 \ldots m}+\frac{2(m+n+2) a_{0} V^{m-1} a_{n}}{1.2 .3 \ldots(m-1)}\right\}
$$

which, by means of (4), reduces to

$$
(-)^{m+1} t^{-n-m-3} \frac{V^{m} \partial_{x} a_{n}}{1.2 .3 \ldots m}
$$

Hence $\quad \partial_{x} \alpha_{n}=-t^{-n-3} \partial_{x} a_{n}+t^{-n-4} V \partial_{x} a_{n}-t^{-n-5} \frac{V^{2} \partial_{x} a_{n}}{1.2}+\ldots$,

or, more concisely,

$$
\partial_{x} \alpha_{n}=-t^{-n-3}\left(e^{-\frac{V}{t}}\right) \partial_{x} a_{n}
$$

But $\quad \partial_{x} a_{n}=(n+3) a_{n+1}$, and $\partial_{x} \alpha_{n}=t \partial_{y} \alpha_{n}=(n+3) t \alpha_{n+1}$,

and therefore

or

$$
\begin{gathered}
(n+3) t a_{n+1}=-(n+3) t^{-n-3}\left(e^{-\frac{V}{t}}\right) a_{n+1}, \\
a_{n+1}=-t^{-n-4}\left(e^{-\frac{V}{t}}\right) a_{n+1} .
\end{gathered}
$$

The theorem is easily seen to be true, for $n=0,1,2$, and is thus proved to be true universally.

I will now return to the point at which I left off in my previous lecture. We saw that the exactitude of the formula

$$
(w ; i, j)-(w-1 ; i+1, j)
$$

for the number of pure reciprocants of the type $w ; i, j$ could not be inferred with certainty unless we were able to prove that the $(w-1 ; i+1, j)$ linear equations between the coefficients of $R$, found by equating $V R$ to zero, were all of them independent. A similar difficulty presents itself in the proof of the corresponding formula $(w ; i, j)-(w-1 ; i, j)$ in the invariantive theory; but in that case I succeeded in making out a proof of the independence of the equations of condition founded on the fact that $\Omega^{m} O^{m} I$ is a numerical multiple of $I$, where $I$ is any invariant, and $\Omega, O$ are the well-known operators

$$
\begin{aligned}
& a_{0} \partial_{a_{1}}+2 a_{1} \partial_{a_{2}}+3 a_{2} \partial_{a_{3}}+\ldots+j a_{j-1} \partial_{a_{j}} \\
& a_{j} \partial_{a_{j-1}}+2 a_{j-1} \partial_{a_{j-2}}+3 a_{j-2} \partial_{a_{j-3}}+\ldots+j a_{1} \partial_{a_{0}} .
\end{aligned}
$$


I have since discovered a second proof of the theorem for invariants which, though very interesting, is less simple than my first; but neither of these methods can be extended to the case of reciprocants.

It was suggested by Capt. MacMahon that the fact that $V^{m} \partial_{x}{ }^{m} R$ is a numerical multiple of $a^{m} R$ ought to lead to a proof of the theorem for reciprocants similar to that obtained for invariants by my first method, alluded to above, but this I find is not the case; and indeed it is capable of being shown a priori that it cannot lead to a proof. One great distinction between the two theories, which is fatal to the success of the proposed method, is well worthy of notice.

If

$$
(w ; i, j)-(w-1 ; i, j)=>0
$$

(I shall sometimes call this positive), then

$$
\left(w^{\prime} ; i, j\right)-\left(w^{\prime}-1 ; i, j\right)=>0
$$

for all values of $w^{\prime}$ less than $w$; the condition that this difference, say $\Delta(w ; i, j)$ shall be positive being simply that $i j-2 w$ is positive (that is, $i j-2 w=>0)$. This is not the case with the difference

$$
(w ; i, j)-(w-1 ; i+1, j)
$$

say $E(w ; i, j)$; it by no means follows that if this is positive for a given value of $w(i, j$ being kept constant), it will be so for any inferior value of $w$.

We may illustrate geometrically the condition $i j-2 w=>0$, which holds when $\Delta(w ; i, j)$ is non-negative.

Let $(i, j)$ be co-ordinates of a point in a plane and draw the positive branch of the rectangular hyperbola

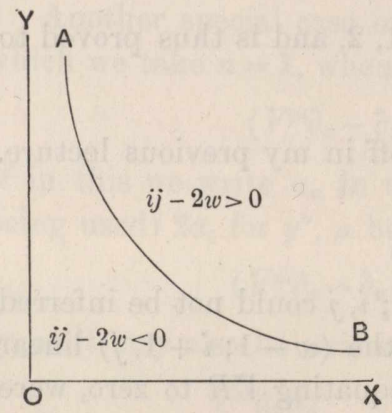

$$
i j-2 w=0 \text {. }
$$

Then, $i j-2 w<0$ for all points in the area $Y O X B A$ between the curve and its asymptotes; but for points on the curve $A B$,

$$
i j-2 w=0 \text {, }
$$

and for all points of the infinite area on the side of $A B$ remote from the origin,

$$
i j-2 w>0 .
$$

Thus, for all points which lie either on or beyond the curve $A B, \Delta(w ; i, j)$ is non-negative, and for all points between the curve and the asymptotes $\Delta(w ; i, j)$ is non-positive.

We have here considered $w$ as constant and $i, j$ as variable, but in the case where all three are variable we should have to consider the hyperbolic paraboloid

$$
i j-2 w=0,
$$

of which the curve $A B$ is a section, by the plane $w=$ const.; and the condition 
of $\Delta(w ; i, j)$ being non-negative or non-positive depends on the variable point $(i, j, w)$ lying in the one case on or beyond the surface, and in the other between the surface and the planes of reference.

The function of $i, j, w$, whose positive or negative sign determines in like manner that of $E(w ; i, j)$, cannot be linear in $w$. What its form is, or whether it is an Algebraical or Transcendental function, no one at present can say. Indeed, except for the light shed on the subject by the Algebraical Theory of Invariants, it would have been exceedingly difficult (as I know from vain efforts made by myself and others in Baltimore) to prove the much simpler theorem that $\Delta(w ; i, j)$ is positive (that is, non-negative) when $i j-2 w$ is so. It amounts to the assertion that the coefficient of $a^{i} x^{w}$ in the expansion of

$$
\frac{1-x}{(1-a)(1-a x)\left(1-a x^{2}\right) \ldots\left(1-a x^{j}\right)}
$$

is always non-negative, provided that $i j-2 w$ is non-negative.

This is a theorem of great importance in the ordinary Theory of Invariants, and may be seen to be a consequence of the fact, which I have proved, that (using $[w ; i, j]$ to denote a function of the type $w ; i, j$ having its full number of arbitrary coefficients) there are no linear connections between the coefficients of $\Omega[w ; i, j]$ when $i j-2 w=>0$; but no one, as far as I know, has ever found a direct proof of it.

Viewing the connection between the two theories of Invariants and Reciprocants, I think it desirable to recapitulate with some improvements the proof, given in the Phil. Mag. for March, 1878, of the theorem that the number of linearly independent invariants of the type $w ; i, j$ is exactly $\Delta(w ; i, j)$ when this quantity is positive, and exactly zero when it is 0 or negative.

As regards reciprocants, at present we can only say that the number of linearly independent ones of the type $w ; i, j$ is never less than $E(w ; i, j)$, leaving to some gifted member of the class to prove or disprove that the first is always exactly equal to the second. The exact theorem to be proved in the theory of invariants is as follows:

If $i j-2 w=>0$, the number of linearly independent invariants of the type $w ; i, j$ is $\Delta(w ; i, j)$.

If $i j-2 w<0$, the number of such invariants is zero; that is, there are none. The proof is made to depend on the properties of

and of

$$
\Omega=a_{0} \partial_{a_{1}}+2 a_{1} \partial_{a_{2}}+3 a_{2} \partial_{a_{3}}+\ldots+j a_{j-1} \partial_{a_{j}}
$$

If $U$ be any homogeneous isobaric function of degree $i$ and weight $w$ in the letters $a_{0}, a_{1}, a_{2}, \ldots a_{j}$, it is easy to prove that

$$
(\Omega O-O \Omega) U=(i j-2 w) U,
$$


and consequently, if $U$ is an invariant $I$, so that $\Omega I=0$,

$$
\Omega O I=(\ddot{j}-2 w) I .
$$

I call $i j-2 w$ the excess and denote it by $\eta$, and shall first show that if $\eta$ is negative $I=0$; that is, there exists no invariant with a negative excess. This will prove that when $\Delta(w ; i, j)$ is negative, that is, when

$$
(w-1 ; i, j)>(w ; i, j)
$$

the number of independent functions of the coefficients of $[w ; i, j]$ which appear in $\Omega[w ; i, j]$ is exactly equal to $(w ; i, j)$, which is the number of the coefficients themselves. Clearly it cannot be greater; for, no matter what the number of linear functions of $n$ quantities may be, only $n$ at the utmost can be independent; there might be fewer, there cannot possibly be more. The complete theorem is that the number of independent coefficients in $\Omega[w ; i, j]$ is the subdominant of two numbers: one the number of terms of the type $w ; i, j$, the other the number of terms of the type $w-1 ; i, j$.

N.B. That one of two numbers which is not greater than the other is called the subdominant.

\section{LECTURE XI.}

We may write for the Annihilator of an Invariant

$$
\Omega=a_{0} \dot{a}_{1}+2 a_{1} \dot{a}_{2}+3 a_{2} \dot{a}_{3}+\ldots+j a_{j-1} \dot{a}_{j}
$$

and for its opposite

$$
O=j a_{1} \dot{a}_{0}+(j-1) a_{2} \dot{a}_{1}+(j-2) a_{3} \dot{a}_{2}+\ldots+a_{j} \dot{a}_{j-1},
$$

where the pointed letters $\dot{a}_{0}, \dot{a}_{1}, \dot{a}_{2}, \ldots \dot{a}_{j}$ stand for the partial differential operators

$$
\partial_{a_{0}}, \partial_{a_{1}}, \partial_{a_{2}}, \ldots \partial_{a_{j}}
$$

Suppose $\Omega$ and $O$ to operate on any function $U\left(a_{0}, a_{1}, a_{2}, \ldots a_{j}\right)$; then

$$
\begin{array}{ll} 
& \Omega O U=(\Omega .0+\Omega * 0) U \\
\text { and } & O \Omega U=(0 . \Omega+0 * \Omega) U,
\end{array}
$$

where the full stop between $O$ and $\Omega$ signifies multiplication, and the asterisk operation on the unpointed letters only. Thus,

$$
\Omega .0=0 . \Omega \text {, }
$$

and, consequently, $\quad(\Omega O-O \Omega) U=(\Omega * O-O * \Omega) U$.

Now,

$$
\Omega * O U=\left\{1 \cdot j a_{0} \dot{a}_{0}+2(j-1) a_{1} \dot{a}_{1}+3(j-2) a_{2} \dot{a}_{2}+\ldots+j \cdot 1 a_{j-1} \dot{a}_{j-1}\right\} U,
$$
and

$$
O * \Omega U=\left\{1 \cdot j a_{1} \dot{a}_{1}+2(j-1) a_{2} \dot{a}_{2}+\ldots+(j-1) 2 a_{j-1} \dot{a}_{j-1}+j .1 a_{j} \dot{a}_{j}\right\} U,
$$


whence we readily obtain

$$
\begin{aligned}
(\Omega U-O \Omega) U=j\left(a_{0} \dot{a}_{0}+a_{1} \dot{a}_{1}\right. & \left.+a_{2} \dot{a}_{2}+\ldots+a_{j} \dot{a}_{j}\right) U \\
& -2\left(a_{1} \dot{a}_{1}+2 a_{2} \dot{a}_{2}+3 a_{3} \dot{a}_{3}+\ldots+j a_{j} \dot{a}_{j}\right) U .
\end{aligned}
$$

Introducing the conditions of homogeneity and isobarism, namely,

$$
\left(a_{0} \dot{a}_{0}+a_{1} \dot{a}_{1}+a_{2} \dot{a}_{2}+\ldots+a_{j} \dot{a}_{j}\right) U=i U
$$

and

$$
\left(a_{1} \dot{a}_{1}+2 a_{2} \dot{a}_{2}+3 a_{3} \dot{a}_{3}+\ldots+j a_{j} \dot{a}_{j}\right) U=w U,
$$

where $i$ and $w$ denote the degree and weight of $U$, supposed now to be a rational integral homogeneous and isobaric function (or, to avoid a tedious periphrasis, say a gradient), we see that if the complete type of the gradient $U$ is $w ; i, j$,

where $\eta$ is the excess.

$$
(\Omega O-O \Omega) U=(i j-2 w) U=\eta U,
$$

Since the operation of 0 increases the weight of the operand by unity, but does not alter either its degree or its extent, it is clear that the type of $O^{\theta} U$ is $w+\theta ; i, j$. The excess of $O^{\theta} U$ is therefore

$$
i j-2(w+\theta)=\eta-2 \theta \text {, }
$$

and the theorem just proved shows that

$$
(\Omega O-O \Omega) O^{\theta} U=(\eta-2 \theta) O^{\theta} U .
$$

From this we pass on to prove that $\Omega O^{q}-O^{q} \Omega$, acting on any gradient as its objective, is equivalent to $q(\eta-q+1) O^{q-1}$; that is, when $q$ is any positive integer, we shall show that

$$
\left(\Omega O^{q}-O^{q} \Omega\right) U=q(\eta-q+1) O^{q-1} U .
$$

The subsequent consideration of a special case of this formula, in which $U$ is replaced by any invariant $I$, will enable us to prove that there can be no invariants for which the excess $i j-2 w$ is negative. Let

$$
O^{q-\theta} \Omega O^{\theta} U=P_{\theta} U
$$

then

$$
O^{q-\theta-1} \Omega O^{\theta+1} U=P_{\theta+1} U \text {, }
$$

and therefore

$$
\left(P_{\theta+1}-P_{\theta}\right) U=O^{q-\theta-1}(\Omega O-O \Omega) O^{\theta} U \text {. }
$$

Substituting in this for

$$
(\Omega O-O \Omega) O^{\theta} U \text { its value }(\eta-2 \theta) 0^{\theta} U \text {, }
$$

we have

$$
\left(P_{\theta+1}-P_{\theta}\right) U=(\eta-2 \theta) O^{q-1} U \text {. }
$$

Hence

$$
\begin{aligned}
\left(P_{q}-P_{0}\right) U & =\left\{\left(P_{1}-P_{0}\right)+\left(P_{2}-P_{1}\right)+\left(P_{3}-P_{2}\right)+\ldots+\left(P_{q}-P_{q-1}\right)\right\} U \\
& =\{\eta+(\eta-2)+(\eta-4)+\ldots+(\eta-2 q+2)\} O^{q-1} U \\
& =q(\eta-q+1) O^{q-1} U .
\end{aligned}
$$

But since $P_{q}=\Omega O^{q}$ and $P_{0}=O^{q} \Omega$, this result may be written

$$
\left(\Omega O^{q}-O^{q} \Omega\right) U=q(\eta-q+1) O^{q-1} U .
$$


If now $U=I$, an invariant, we have $\Omega U=0$, and our formula becomes

$$
\Omega O^{q} I=q(\eta-q+1) O^{q-1} I \text {. }
$$

Writing in succession $q=m, m-1, \ldots 1$, we obtain

$$
\begin{aligned}
& m(\eta-m+1) O^{m-1} I=\Omega O^{m} I \\
& (m-1)(\eta-m+2) O^{m-2}=\Omega O^{m-1} I \\
& (m-2)(\eta-m+3) O^{m-3} I=\Omega O^{m-2} I \\
& \text { 1. } \eta I=\Omega O I .
\end{aligned}
$$

By assigning to $m$ a sufficiently large value we are able to make $O^{m} I$ vanish as well as $\Omega I$; for, the type of $I$ being $w ; i, j$, that of $O^{m} I$ is $w+m ; i, j$. But it is evident that no gradient can have a greater weight than $i j$, the product of its degree and extent, for each term is a product of $i$ letters none of them having a weight greater than $j$. If, then, we suppose that $m=i j-w+1$, the weight of $O^{m} I$ is

Therefore

$$
w+m=i j+1 \text {. }
$$

Again, $\quad \eta-m+1=\ddot{i j}-2 w-(\ddot{j}-w+1)+1=-w$.

If, then, $\eta$ is negative, every term in the series

$$
m(\eta-m+1),(m-1)(\eta-m+2), \ldots 2(\eta-1), 1 . \eta
$$

is negative and can never vanish. Hence we have successively

$$
O^{m-1} I=0, O^{m-2} I=0, \ldots I=0 ;
$$

that is, when $i j-2 w<0$ no invariant of the type $w ; i, j$ exists.

Observe that the elenchus of the demonstration consists in the fact that the successive numerical factors $\eta-m+1, \eta-m+2, \eta-m+3, \ldots \eta$ are all non-zero on account of $\eta$ being negative; but if $\eta$ were positive we should eventually come to a factor $\eta-\mu$ which would be zero, and we could not conclude from $(\mu+1)(\eta-\mu) O^{\mu} I$ being zero that $O^{\mu} I=0$. Since $\eta-(m-1)$ passes from $\eta-(\ddot{j}-w)$ to $\eta$, that is, from $-w$ to $\eta$, it passes through zero when $\eta$ is positive.

The second part of Cayley's completed theorem remains to be proved, namely, that when $i j-2 w=>0$, the number of linearly independent invariants of the type $w ; i, j$ is precisely equal to $\Delta(w ; i, j)$; that is, to

$$
(w ; i, j)-(w-1 ; i, j)
$$

I show this by proving that if $D(w ; i, j)$ is the number in question, keeping $i$ and $j$ constant and taking $w<=\frac{\ddot{j}}{2}$,

$$
D(w ; i, j)+D(w-1 ; i, j)+D(w-2 ; i, j)+\ldots+D(0 ; i, j)
$$

cannot be greater than

$$
\Delta(w ; i, j)+\Delta(w-1 ; i, j)+\Delta(w-2 ; i, j)+\ldots+\Delta(0 ; i, j)
$$


and consequently, since we know that no single $D(w ; i, j)$ can possibly be less than the corresponding $\Delta(w ; i, j)$, it follows that

$$
\begin{aligned}
& D(w ; i, j)+D(w-1 ; i, j)+D(w-2 ; i, j)+\ldots+D(0 ; i, j) \\
= & \Delta(w ; i, j)+\Delta(w-1 ; i, j)+\Delta(w-2 ; i, j)+\ldots+\Delta(0 ; i, j)
\end{aligned}
$$

and, furthermore, that each

$$
D(w ; i, j)=\Delta(w ; i, j)
$$

For if any $D$ were greater than its corresponding $\Delta$, some other $D$ would have to be less, which is impossible.

This principle of reasoning may be illustrated by imagining a row of ballot-boxes and supposing it to be ascertained that no single box contains fewer white balls than black ones. If, then, there are not more white than black balls altogether, the total number of whites must be the same as that of the blacks. And since there are just as many whites as blacks distributed among the ballot-boxes, the number of white and black balls must be the same in each box; for otherwise some box must contain fewer whites than blacks, which is contrary to the hypothesis.

Observe that the sum of these $\Delta$ 's is $(w ; i, j)$; for

$$
\begin{aligned}
& (w ; i, j)-(w-1 ; i, j)+(w-1 ; i, j)-(w-2 ; i, j)+\ldots+(0 ; i, j)-(-1 ; i, j) \\
& =(w ; i, j)-(-1 ; i, j) \\
& (-1 ; i, j)=0 \text {, }
\end{aligned}
$$

since there is no way of composing -1 with parts $0,1,2, \ldots j$. Hence what I have to show is that

$$
D(w ; i, j)+D(w-1 ; i, j)+\ldots+D(1 ; i, j)+D(0 ; i, j)=(w ; i, j)
$$

I want preliminarily to express $\Omega^{q} O^{q} I$ as a multiple of $I^{*}$.

This can be done by a formula previously demonstrated, namely,

which gives

$$
\Omega O^{q} I=q(\eta-q+1) O^{q-1} I,
$$

$$
\Omega^{2} O^{q} I=q(\eta-q+1) \Omega O^{q-1} I=q(\eta-q+1)(q-1)(\eta-q+2) O^{q-2} I ;
$$

similarly

$$
\Omega^{3} O^{q} I=q(\eta-q+1)(q-1)(\eta-q+2)(q-2)(\eta-q+3) O^{q-3} I
$$

and finally, changing the order of the numerical factors,

$$
\Omega^{q} O^{q} I=1.2 .3 \ldots q\{\eta(\eta-1)(\eta-2) \ldots(\eta-q+1)\} I .
$$

This shows that $\Omega^{q} O^{q} I$ and $\grave{a}$ fortiori $O^{q} I$ can never vanish unless $\eta-q+1$ becomes negative.

* The result of operating on $I$ with $O$ and $\Omega$ each $q$ times, the two operations following each other according to any law of distribution whatever, will always be a numerical multiple of $I$; but the value of this multiple will differ for different laws of distribution. 
Suppose now that $I^{q}$ means an invariant of the type $w-q ; i, j$; its excess is $i j-2(w-q)$, and consequently $O^{q} I_{q}$ cannot vanish unless

$$
i j-2(w-q)-q+1
$$

becomes negative, which is impossible. For

$$
\ddot{i j}-2(w-q)-q+1=\ddot{j}-2 w+q+1
$$

and $i j-2 w=>0$ by hypothesis.

By taking $O^{q} I_{q}$ as an image, so to say, of $I_{q}$ we shall be able to obtain a limit to the number of $I_{q}$ 's by obtaining a limit to the number of their images. In fact, taking the image $O^{q} I_{q}$ of each of the $D(w-q ; i, j)$ linearly independent invariants of the type $w-q ; i, j$ (this is what is meant by the $I_{q}$ 's) and giving $q$ all possible values from 0 to $w$ inclusive, the total number of these images is obviously

$$
D(w ; i, j)+D(w-1 ; i, j)+\ldots+D(0 ; i, j)
$$

Each of them will be a gradient of the weight $w-q+q$ (that is, of weight $w$ ), and will consist of terms of weight $w$, degree $i$, and extent $j$. The total number of such terms will be the number of ways of making up $w$ with $i$ of the numbers $0,1,2,3, \ldots j$, or with the usual notation $(w ; i, j)$. If, then, it can be shown that none of these forms are linearly connected, then, inasmuch as they are all functions of the same $(w ; i, j)$ arguments, it will follow that their total number cannot exceed $(w ; i, j)$. That is, we shall have shown that

$$
D(w ; i, j)+D(w-1 ; i, j)+D(w-2 ; i, j)+\ldots+D(0 ; i, j)
$$

cannot exceed

$$
\Delta(w ; i, j)+\Delta(w-1 ; i, j)+\Delta(w-2 ; i, j)+\ldots+\Delta(0 ; i, j)
$$

and by the ballot-box principle, as already stated (inasmuch as no $D$ is less than its corresponding $\Delta$ ), it will follow that each $D$ is the same as the corresponding $\Delta$, and the theorem to be proved is established.

The proof of this independence is easy. For (1) suppose that there is any linear relation between the forms

$$
O^{q} I_{q}, O^{q} I_{q}^{\prime}, O^{q} I_{q}^{\prime \prime}, \ldots,
$$

for each of which the value of $q$ is the same. Denoting these forms by

let the relation in question be

$$
P_{q}, P_{q}^{\prime}, P_{q}^{\prime \prime}
$$

$$
\lambda P_{q}+\lambda^{\prime} P_{q}^{\prime}+\lambda^{\prime \prime} P_{q}^{\prime \prime}+\ldots=0 .
$$

Then

$$
\lambda \Omega^{q} P_{q}+\lambda^{\prime} \Omega^{q} P_{q}^{\prime}+\lambda^{\prime \prime} \Omega^{q} P_{q}^{\prime \prime}+\ldots=0 .
$$

But each argument $\Omega^{q} P_{q}$ is of the form $\Omega^{q} O^{q} I_{q}$, and since this is equal 
to $I_{q}$ multiplied by a number which does not vanish*, we have a linear relation between $I_{q}, I_{q}^{\prime}, I_{q}^{\prime \prime}, \ldots$, namely

$$
\lambda I_{q}+\lambda^{\prime} I_{q}^{\prime}+\lambda^{\prime \prime} I_{q}^{\prime \prime}+\ldots=0 ;
$$

that is, the $I_{q}$ 's would not be linearly independent, contrary to hypothesis. Thus the images $\left(O^{q} I_{q}, O^{q} I_{q}^{\prime}, O^{q} I_{q}^{\prime \prime} \ldots\right)$ belonging to invariants of the same type $w-q ; i, j$ cannot be linearly connected.

(2) I say that the images of invariants of different types cannot be linearly connected. For let $q, q^{\prime}, q^{\prime \prime}, \ldots$ arranged in descending order of magnitude, be the different values of $q$ in the images supposed to be linearly related. The result of operating with $\Omega^{q}$ on any image of the form $O^{q^{\prime}} I_{q^{\prime}}$ is to bring it to the form $\Omega^{q-q^{\prime}} \Omega^{q^{\prime}} O^{q^{\prime}} I_{q^{\prime}}$, which is a multiple of $\Omega^{q-q^{\prime}} I_{q^{\prime}}$, and therefore vanishes. But $\Omega^{q}$, acting on any of the images $O^{q} I_{q}, O^{q} I_{q}^{\prime}, \ldots$, will, as we have seen, bring back the multiple of $I_{q}$; thus the operation of $\Omega^{q}$ on the supposed relation will give a linear equation connecting $I_{q}, I_{q}^{\prime}, I_{q}^{\prime \prime}, \ldots$, and for the same reason as before this is impossible. Hence there can be no linear relation whatever between the images of the invariants whose types extend from $w ; i, j$ to $0 ; i, j$, and the number of these images will accordingly be not greater than $(w ; i, j)$, as was to be proved.

It is well worthy of notice that $D(w ; i, j)$ may be zero, but obviously cannot be negative, as it denotes a number of things which may have any value from zero upwards. Hence follows a remarkable theorem in the pure theory of partitions which it would be extremely difficult to prove from first principles, namely, that the difference between the two partition numbers

$$
(w ; i, j)-(w-1 ; i, j)
$$

can never be negative when $i j-2 w=>0$. It may be zero, but cannot be less than zero. This explains what I said about the hyperbolic paraboloid $i j-2 w=0$, where $i, j, w$ are treated as co-ordinates of a point in space. We might call the value of $(w ; i, j)-(w-1 ; i, j)$ the density of any point $i, j, w$, and the theorem may then be expressed by saying that at points within or upon the hyperbolic paraboloid the density can never be negative; for points outside this surface it can never be positive.

As regards the analogous formula in the Theory of Reciprocants

$$
(w ; i, j)-(w-1 ; i+1, j),
$$

we do not know that any algebraical surface can be constructed which will enable us to discriminate between the cases in which this difference, say $E(w ; i, j)$, is positive or negative. Should such a surface exist, its equation must contain $w$ in a higher degree than the first. Supposing that the above

* In fact, remembering that the excess of the type $w-q ; i, j$ is $i j-2(w-q)=\eta+2 q$, we find in which both $\eta$ and $q$ are positive integers.

$$
\Omega^{q} O^{q} I_{q}=1.2 .3 \ldots q\{(\eta+2 q)(\eta+2 q-1) \ldots(\eta+q+1)\} I_{q},
$$

s IV. 
formula represents the actual number of reciprocants, it will follow (and this is confirmed by experience) that there can be no reciprocants to a type of negative excess. For

$$
\begin{aligned}
& (w ; i, j)-(w-1 ; i+1, j) \\
= & (w ; i, j)-(w-1 ; i, j)-[(w-1 ; i+1, j)-(w-1 ; i, j)] \\
= & (w ; i, j)-(w-1 ; i, j)-(w-i-2 ; i+1, j-1) .
\end{aligned}
$$

But if $i j-2 w$ is negative, $(w ; i, j)-(w-1 ; i, j)$ is zero or negative. Hence $(w ; i, j)-(w-1 ; i+1, j)$ is non-positive.

For satisfied invariants (those ordinarily so called) $w=\frac{i j}{2}$, and the formula for their number becomes $\left(\frac{\dddot{j}}{2} ; i, j\right)-\left(\frac{\ddot{j}}{2}-1 ; i, j\right)$.

As these form a well-defined class apart, it would have seemed very natural to begin with them in endeavouring to establish the theorem, reserving the theory of unsatisfied invariants (sources of covariants) for future consideration. But to all appearance it would have been very difficult, if not impossible, to have succeeded in dealing with them alone.

This is another example of the law in Heuristic that the whole is easier of deglutition than its part.

\section{LECTURE XII.}

Before proceeding further with the development of the pure analytical theory of reciprocants, it may be useful to point out some instances of its relations and applications to geometrical questions.

Using $y_{1}, y_{2}, y_{3}, \ldots y_{n}$ to denote the successive derivatives of $y$ with respect to $x^{*}$, let the complete primitive of the differential equation

be

$$
\begin{aligned}
& F\left(x, y, y_{1}, y_{2}, \ldots y_{n}\right)=0 \\
& \phi(x, y, \lambda, \mu, \nu, \ldots)=0 .
\end{aligned}
$$

We can in general so determine the $n$ constants $\lambda, \mu, \nu, \ldots$ that the curve $\phi$ may pass through $n$ given points, and if we take these to be consecutive points on the curve

$$
\Phi(x, y)=0
$$

$\phi$ and $\Phi$ will have a contact of the $(n-1)$ th order at a given point of $\Phi$. In order that the curves may have a contact of the $n$th order at a point

* In future $y_{1}, y_{2}, y_{3}, \ldots y_{n}$ will always have this meaning, the derivatives of $x$ with respect to $y$ will be denoted by $x_{1}, x_{2}, x_{3}, \ldots$, and whenever the letters $t, a, b, c, \ldots$ are used they will stand for $y_{1}, \frac{y_{2}}{1.2}, \frac{y_{3}}{1.2 .3}, \frac{y_{4}}{1.2 .3 .4}, \ldots$ respectively. 
whose abscissa is $x$, the ordinates of $\Phi$ and $\phi$ at that point and their 1st, 2 nd, ... nth derivatives with respect to $x$ must be the same for both curves. But at every point of $\phi$ its differential equation

$$
F\left(x, y, y_{1}, y_{2}, \ldots y_{n}\right)=0
$$

has to be satisfied, and therefore the $x, \dot{y}, y_{1}, y_{2}, \ldots y_{n}$ of any point on $\Phi$, at which contact of the $n$th order with $\phi$ is possible, must also satisfy the same equation.

Now, suppose that for $x$ and $y$ we substitute given functions of them, $X$ and $Y$; the curves $\phi$ and $\Phi$ become

$$
\phi(X, Y, \lambda, \mu, \nu, \ldots)=0 \text { and } \Phi(X, Y)=0 .
$$

Contact of the $n$th order with the transformed $\phi$ will therefore be possible at any point of the transformed $\Phi$ for which

$$
F\left(X, Y, Y_{1}, Y_{2}, \ldots Y_{n}\right)=0,
$$

where $Y_{1}, Y_{2}, Y_{3}, \ldots Y_{n}$ are the derivatives of $Y$ with respect to $X$.

But, unless the function $F$ and the substitutions $X=f_{1}(x, y), Y=f_{2}(x, y)$ are so related that the transformed differential equation

$$
F\left(X, Y, Y_{1}, Y_{2}, \ldots Y_{n}\right)=0
$$

is identical with the untransformed one, the property marked by the contact of the transformed curves will not be identical with that marked by the contact of the untransformed ones.

For example, let $F=y_{2}$; then the relation between $\phi \equiv y+\lambda x+\mu=0$ (the complete primitive of $y_{2}=0$ ) and an arbitrary curve $\Phi$ is that the constants $\lambda$ and $\mu$ may be so chosen that the line $y+\lambda x+\mu=0$ may have a contact of the second order at any point of $\Phi$ for which $y_{2}=0$; and the property marked is an inflexion on $\Phi$. But if we make the substitution $X=x^{2}, Y=y^{2}$, so that the differential equation $y_{2}=0$ is transformed into $\left(\frac{d}{d x^{2}}\right)^{2} y^{2}=0$ and its complete primitive into $y^{2}+\lambda x^{2}+\mu=0$, it will still be possible so to choose $\lambda$ and $\mu$ that $y^{2}+\lambda x^{2}+\mu=0$ may have a contact of the second order at any point of an arbitrary curve for which $\left(\frac{d}{d x^{2}}\right)^{2} y^{2}=0$, but the property marked, instead of being an inflexion, will be a contact of the second order with a conic having a pair of conjugate diameters coincident with the co-ordinate axes.

The property remains unaltered when the co-ordinate axes are interchanged, and therefore the differential equation $\left(\frac{d}{d x^{2}}\right)^{2} y^{2}=0$ will be identical with $\left(\frac{d}{d y^{2}}\right)^{2} x^{2}=0$, in which the variables $x$ and $y$ have changed places. The $24-2$ 
identity of the two differential equations is easily, verified, for

$$
\begin{aligned}
\left(\frac{d}{d x^{2}}\right)^{2} y^{2} & =\frac{1}{2 x} \cdot \frac{d}{d x}\left(\frac{y}{x} \cdot \frac{d y}{d x}\right)=\frac{1}{2 x} \cdot\left\{\frac{y}{x} \cdot \frac{d^{2} y}{d x^{2}}+\frac{1}{x}\left(\frac{d y}{d x}\right)^{2}-\frac{y}{x^{2}} \cdot \frac{d y}{d x}\right\} \\
& =\frac{1}{2 x^{3}}\left(x y y_{2}+x y_{1}{ }^{2}-y y_{1}\right)
\end{aligned}
$$

so that the differential equation may be written

$$
x y y_{2}+x y_{1}^{2}-y y_{1}=0 \text {. }
$$

Interchanging $x$ and $y$ in this, we have

$$
y x x_{2}+y x_{1}^{2}-x x_{1}=0,
$$

in which, if we write $x_{1}=\frac{d x}{d y}=\frac{1}{y_{1}}$, and $x_{2}=\frac{d^{2} x}{d y^{2}}=-\frac{y_{2}}{y_{1}{ }^{3}}$, it follows immediately that

$$
y x x_{2}+y x_{1}^{2}-x x_{1}=-\frac{1}{y_{1}^{3}}\left(x y y_{2}+x y_{1}^{2}-y y_{1}\right)
$$

and the identity in question is established.

Such a form as the above, which merely acquires an extraneous factor when the variables are interchanged, might be called a reciprocant, if it were not convenient to restrict the use of the word to forms in which the variables $x$ and $y$ do not appear explicitly. With this limitation, the geometrical property indicated by the evanescence of a reciprocant will be independent of the position of the origin, but not in general independent of the directions of the co-ordinate axes. Thus, we may prove that the equation

$$
2 y_{1} y_{3}-3 y_{2}^{2}=0
$$

indicates the possibility of 4-point contact with a hyperbola whose asymptotes are parallel to the co-ordinate axes. To do this it is sufficient to show that its complete primitive is the equation to such a hyperbola.

Writing the equation in the form

we see that its first integral is

$$
\frac{y_{3}}{y_{2}}=\frac{3}{2} \cdot \frac{y_{2}}{y_{1}}
$$

$$
\log y_{2}=\frac{3}{2} \log y_{1}+\text { const. }
$$

or, when prepared for a second integration,

$$
-\frac{1}{2} \cdot y_{1}^{-\frac{3}{2}} y_{2}=\lambda
$$

Hence

$$
\begin{aligned}
y_{1}{ }^{-\frac{1}{2}} & =\lambda x+\mu, \\
y_{1} & =(\lambda x+\mu)^{-2},
\end{aligned}
$$

and finally we obtain the complete primitive

$$
\lambda(\nu-y)=(\lambda \dot{x}+\mu)^{-1}
$$

which proves the proposition. 
With the notation previously explained, in which $y_{1}=t, y_{2}=2 a, y_{3}=6 b$, the differential equation is $b t-a^{2}=0$. We have therefore proved that at all points of a general curve for which the Schwarzian $\left(b t-a^{2}\right)$ vanishes, 4-point contact with a hyperbola whose asymptotes are parallel to the co-ordinate axes is possible.

We now consider the important case in which the conditioning differential equation remains unchanged when the axes are orthogonally transformed, and is therefore found by equating to zero an orthogonal reciprocant. The simplest example of this class of equations is that which marks the points of maximum or minimum curvature on a curve. Since these points are points of 4-point contact with a circle, the conditioning differential equation will be that of the circle

$$
(x+\lambda)^{2}+(y+\mu)^{2}+\nu=0 .
$$

Differentiating this three times in succession, we have

$$
\begin{array}{r}
x+\lambda+(y+\mu) t=0 \\
1+t^{2}+2 a(y+\mu)=0 \\
a t+b(y+\mu)=0
\end{array}
$$

Eliminating $\mu$ from the last two of these equations, $y$ will disappear at the same time, and the condition for points of maximum or minimum curvature is found to be

$$
2 a^{2} t-b\left(1+t^{2}\right)=0 \text {. }
$$

In Salmon's Higher Plane Curves (2nd edition, p. 357) the "aberrancy of curvature" is given by the formula

$$
\tan \delta=y_{1}-\frac{\left(1+y_{1}^{2}\right) y_{3}}{3 y_{2}{ }^{2}}=t-\frac{\left(1+t^{2}\right) b}{2 a^{2}}
$$

The above differential equation is therefore equivalent to $\delta=0$.

If we differentiate the radius of curvature $\rho=\frac{\left(1+y_{1}^{2}\right)^{\frac{3}{2}}}{y_{2}}=\frac{\left(1+t^{2}\right)^{\frac{3}{2}}}{2 a}$, we find

$$
\frac{d \rho}{d x}=\frac{6 a^{2} t\left(1+t^{2}\right)^{\frac{1}{2}}-3 b\left(1+t^{2}\right)^{\frac{3}{2}}}{2 a^{2}}=3\left(1+t^{2}\right)^{\frac{1}{2}} \tan \delta=3 \tan \delta \cdot \frac{d s}{d x} .
$$

Hence it follows that

$$
\tan \delta=\frac{1}{3} \cdot \frac{d \rho}{d s}
$$

The conditioning equation for points at which $\frac{d \rho}{d s}$ or $\tan \delta$ is a maximum or minimum is $\frac{d^{2} \rho}{d s^{2}}=0$; or the same condition may be expressed by

Now

$$
\frac{d \tan \delta}{d x}=0
$$

$$
\frac{d \tan \delta}{d x}=\frac{d}{d x}\left\{t-\frac{b\left(1+t^{2}\right)}{2 a^{2}}\right\}=2 a-\frac{2 c\left(1+t^{2}\right)}{a^{2}}-\frac{2 a b t}{a^{2}}+\frac{3 b^{2}\left(1+t^{2}\right)}{a^{3}}
$$


is an orthogonal reciprocant, for it can be expressed in terms of legitimate combinations of $1+t^{2}$, which is an orthogonal reciprocant of even character, with the three orthogonal reciprocants of odd character,

$$
a, b\left(1+t^{2}\right)-2 a^{2} t, c\left(1+t^{2}\right)-5 a b t+5 a^{3} .
$$

In fact, the above expression for $\frac{d \tan \delta}{d x}$, when multiplied by $a^{3}$ to clear of fractions, becomes

$$
\begin{aligned}
2 a^{4}-2 a^{2} b t & +3 b^{2}\left(1+t^{2}\right)-2 a c\left(1+t^{2}\right) \\
= & \frac{3}{1+t^{2}}\left\{b\left(1+t^{2}\right)-2 a^{2} t\right\}^{2}+\frac{12 a^{4}}{1+t^{2}}-2 a\left\{c\left(1+t^{2}\right)-5 a b t+5 a^{3}\right\},
\end{aligned}
$$

where the right-hand side is a linear function of orthogonal reciprocants of the same (even) character, so that the combination is legitimate.

Quantities such as $\rho, \frac{d \rho}{d s}, \frac{d^{2} \rho}{d s^{2}}, \ldots$, or $\rho, \frac{d \rho}{d \phi}, \frac{d^{2} \rho}{d \phi^{2}}, \ldots$, where $d \phi$ is the angle subtended by the arc $d s$ at the centre of curvature, have values independent of the particular position of the co-ordinate axes (supposed rectangular), and consequently these values, expressed in terms of $t, a, b, c, \ldots$ will be absolute orthogonal reciprocants. A differential equation expressing the condition that any one of these quantities vanishes, or that any one of them has a maximum or minimum value, will also be independent of the position of the rectangular axes, and must therefore be expressible in the form of an orthogonal reciprocant equated to zero.

Mr Hammond remarks that, since the radii of curvature at corresponding points of a curve and its evolute are $\rho$ and $\frac{d \rho}{d \phi}$, the radius of curvature of its $n$th evolute is $\frac{d^{n} \rho}{d \phi^{n}}$. The radius of curvature of the $n$th evolute of any $n$th involute of a circle is constant, and, consequently, the differential equation of an $n$th involute to a circle is

Writing this in the form

$$
\frac{d^{n+1} \rho}{d \phi^{n+1}}=0
$$

$$
\left(\frac{1+t^{2}}{a} \cdot \frac{d}{d x}\right)^{n+1} \cdot \frac{\left(1+t^{2}\right)^{\frac{3}{2}}}{a}=0
$$

to which it is easily reduced, since

$$
\frac{d}{d \phi}=\rho \cdot \frac{d}{d s}=\frac{\rho}{\left(1+t^{2}\right)^{\frac{1}{2}}} \cdot \frac{d}{d x}=\frac{\left(1+t^{2}\right)}{2 a} \cdot \frac{d}{d x},
$$

we see by what precedes that the left-hand member of the differential equation is an orthogonal reciprocant.

As an example of the class of singularities which next presents itself for consideration, let us find the differential condition which holds at points of 
contact of the fourth order with a common parabola. This condition is expressible by the differential equation whose complete primitive is

$$
(y+\kappa x)^{2}+2 \lambda x+2 \mu y+\nu=0 .
$$

Differentiating three times in succession, we obtain

$$
\begin{array}{r}
(y+\kappa x)(t+\kappa)+\lambda+\mu t=0, \\
2 a(y+\kappa x+\mu)+(t+\kappa)^{2}=0, \\
b(y+\kappa x+\mu)+a(t+\kappa)=0 .
\end{array}
$$

The arbitrary constants $\nu$ and $\lambda$ do not appear in the last two of these equations, from which, if we eliminate $\mu$, the variables $x$ and $y$ disappear at the same time, and we find

$$
2 a^{2}-b(t+\kappa)=0 .
$$

A final differentiation and elimination give

$$
\begin{array}{r}
10 a b-4 c(t+\kappa)=0, \\
4 a c-5 b^{2}=0 .
\end{array}
$$

Points of 5-point contact with a parabola are therefore indicated by the evanescence of the pure reciprocant $4 a c-5 b^{2}$. And in general the differential equation $R=0$, where $R$ is any pure reciprocant, indicates a property of a curve which may be called a descriptive singularity, since it is totally unaffected by the arbitrary choice of any two lines on the plane for the axes of co-ordinates. For it was proved in Lecture IX of the present course that if $i$ be the degree and $\mu$ the characteristic of $R$, the substitution of $l y+m x+n$ for $x$ and $l^{\prime} y+m^{\prime} x+n^{\prime}$ for $y$ changes $R$ into $\left(l^{\prime} m-l m^{\prime}\right)^{i}(l t+m)^{-\mu} R$, so that the differential equation $R=0$ and the geometrical property corresponding to it are left unchanged by the substitution.

Six-point contact with a cubical parabola is another example of a descriptive singularity. Its defining differential equation may be written in any of the following forms:

$$
\begin{gathered}
45 y_{2}{ }^{3} y_{5}{ }^{2}-450 y_{2}{ }^{2} y_{3} y_{4} y_{5}+192 y_{2}{ }^{2} y_{4}{ }^{3}+400 y_{2} y_{3}{ }^{3} y_{5}+165 y_{2} y_{3}{ }^{2} y_{4}{ }^{2}-400 y_{3}{ }^{4} y_{4}=0 \\
125 a^{3} d^{2}-750 a^{2} b c d+256 a^{2} c^{3}+500 a b^{3} d+165 a b^{2} c^{2}-300 b^{4} c=0 \\
5\left(9 y_{2}{ }^{2} y_{5}-45 y_{2} y_{3} y_{4}+40 y_{3}^{3}\right)^{2}+64\left(3 y_{2} y_{4}-5 y_{3}{ }^{2}\right)^{3}=0 \\
125\left(a^{2} d-3 a b c+2 b^{3}\right)^{2}+4\left(4 a c-5 b^{2}\right)^{3}=0
\end{gathered}
$$

or, if we make $a^{2} d-3 a b c+2 b^{3}=A$ and $a c-\frac{5}{4} b^{2}=M$, the equation may be put in the form

$$
\left(\frac{A}{16}\right)^{2}+\left(\frac{M}{5}\right)^{3}=0
$$

In the theory of Binary Forms, when the numerical parameter $\kappa$ in

$$
\left(a^{2} d-3 a b c+2 b^{3}\right)^{2}+\kappa\left(a c-b^{2}\right)^{3}
$$


is so chosen that the highest powers of $b$ cancel each other, the form divides by $a^{2}$ and gives the Discriminant of the Cubic

$$
a^{2} d^{2}-6 a b c d+4 b^{3} d+4 a c^{3}-3 b^{2} c^{2} .
$$

In the parallel theory of Reciprocants the form

$$
125 A^{2}+256 M^{3}
$$

is divisible by $a$ (instead of by $a^{2}$ ), giving

$$
125 a^{3} d^{2}-750 a^{2} b c d+500 a b^{3} d+256 a^{2} c^{3}+165 a b^{2} c^{2}-300 b^{4} c
$$

which may be called the Quasi-Discriminant.

A complete discussion of the differential equation

$$
A^{2}+\kappa M^{3}=0
$$

is reserved for the next ensuing lecture, in the course of which it will appear that the Quasi-Discriminant equated to zero is the differential equation of the cubical parabola.

\section{LECTURE XIII.}

We may integrate the general homogeneous equation in reciprocants extending to $d$, inclusive, as follows:

$$
\text { Calling } \quad a c-\frac{5}{4} b^{2}=M \text { and } a^{2} d-3 a b c+2 b^{3}=A \text {, }
$$

the equation in question will be of the form

But if we write

$$
\begin{gathered}
A^{2}+\kappa M^{3}=0 . \\
\beta=\Lambda \alpha^{\lambda},
\end{gathered}
$$

where $\beta, \alpha$ are general linear functions of the co-ordinates, say

$$
y+m x+n, y+m^{\prime} x+n^{\prime}
$$

we may eliminate the five constants $m, n, m^{\prime}, n^{\prime}, \Lambda$, and the result will evidently be a pure reciprocant extending to $d$, inclusive, and, being homogeneous and isobaric, can only be of the form

$$
A^{2}+\kappa M^{3}=0
$$

so that it remains only to determine $\kappa$ in terms of $\lambda$, or, which is the same thing, $\lambda$ in terms of $\kappa$.

The solution $\beta=\Lambda \alpha^{\lambda}$ implies $\alpha=\Lambda^{-\frac{1}{\lambda}} \beta^{\frac{1}{\lambda}}$. Hence the equation between $M$ and $A$ must be of the form

$$
\theta\{(\lambda+p)(p \lambda+1)\}^{i} M^{3}+\{(\lambda+q)(q \lambda+1)\}^{j} A^{2}=0,
$$

where $\theta$ is a constant, for otherwise there would be more than one general solution to it. It only remains then to determine the values of $p, q, \theta, i, j$, which may be affected by considering the particular solution $y=x^{\lambda}$. 
When $\lambda=2, M$ and $A$ both vanish, and if $\lambda=2+\epsilon$, where $\epsilon$ is an infinitesimal, $M$ and $A$ will each be of the same order as $\epsilon$ (that the first power of $\epsilon$ does not vanish in $M$ or $A$ may be easily verified). Hence $2+q+\epsilon$ is of the order $\epsilon$, and therefore $q=-2$ and $j=1$.

When $\lambda=-1+\epsilon, M$ remains finite and $A$ is of the order $\epsilon$. Hence $p=1$ and $i=1$. Thus, the equation is

$$
\theta(\lambda+1)^{2} M^{3}+(\lambda-2)(2 \lambda-1) A^{2}=0 .
$$

To find $\theta$, let $\lambda=3$ and $y=x^{3}$; then

$$
a=3 x, b=1, c=0, d=0, M=-\frac{5}{4}, A=2,
$$

so that

$$
-\theta \cdot \frac{5^{3}}{4}+5.4=0, \quad \theta=\frac{16}{25}
$$

and finally

$$
16(\lambda+1)^{2} M^{3}+25\left(2 \lambda^{2}-5 \lambda+2\right) A^{2}=0
$$

has for its integral

$$
\beta=\Lambda \alpha^{\lambda} \text {. }
$$

If $\lambda=\infty$, we may make

$$
y=\left(1+\frac{x}{\lambda}\right)^{\lambda l}=e^{l x}
$$

and, consequently, $\beta=e^{l \alpha}$, which contains five independent arbitrary constants, will be the general integral.

For a parallel method of deducing the Integral of $A^{8}+\kappa \Delta^{3}=0$, where $\Delta$ (our future $A C-B^{2}$ ) is the projective reciprocant whose letters go up to $f$, see Halphen's Thèse sur les Invariants Différentiels, Paris, 1878.

Mr Hammond has succeeded in deducing the equation between $A$ and $M$ from the primitive $\beta=\Lambda \alpha^{\lambda}$ by direct elimination, as shown in what follows. Possibly he, or some other algebraist, may eventually succeed in the more difficult task of obtaining the Differential Equation to $\gamma=\beta^{\lambda} \alpha^{1-\lambda}$ (that is, the linear relation between $A^{8}$ and $\Delta^{3}$ ) by some similar direct process.

Differentiating the equation $\beta \alpha^{-\lambda}=\Lambda$ three times in succession, and observing that, since $\alpha=y+m x+n$ and $\beta=y+m^{\prime} x+n^{\prime}$,

we have

$$
\alpha^{\prime \prime}=\beta^{\prime \prime}=\frac{d^{2} y}{d x^{2}}=y_{2},
$$

$$
\begin{gathered}
\alpha \beta^{\prime}-\lambda \alpha^{\prime} \beta=0, \\
y_{2}(\alpha-\lambda \beta)+(1-\lambda) \alpha^{\prime} \beta^{\prime}=0, \\
y_{3}(\alpha-\lambda \beta)+y_{2}\left\{(2-\lambda) \alpha^{\prime}+(1-2 \lambda) \beta^{\prime}\right\}=0 .
\end{gathered}
$$

From the last two of these three equations we obtain, by eliminating $(\alpha-\lambda \beta)$,

or, writing

$$
y_{3}(1-\lambda) \alpha^{\prime} \beta^{\prime}-y_{2}^{2}\left\{(2-\lambda) \alpha^{\prime}+(1-2 \lambda) \beta^{\prime}\right\}=0 ;
$$

$$
y_{2}=2 a, y_{3}=6 b, 2-\lambda=3 q^{2}, 1-2 \lambda=-3 r^{2}, 1-\lambda=q^{2}-r^{2},
$$


and dividing by $\alpha^{\prime} \beta^{\prime}$, the equation assumes the form

$$
\frac{b}{2 a^{2}}\left(q^{2}-r^{2}\right)=\frac{q^{2}}{\beta^{\prime}}-\frac{r^{2}}{\alpha^{\prime}} .
$$

Differentiating again, remembering that

we find

$$
\alpha^{\prime \prime}=\beta^{\prime \prime}=2 a, \text { and } \frac{d a}{d x}=3 b, \frac{d b}{d x}=4 c,
$$

$$
\frac{4 a c-6 b^{2}}{4 a^{4}}\left(q^{2}-r^{2}\right)=-\frac{q^{2}}{\beta^{\prime 2}}+\frac{r^{2}}{\alpha^{\prime 2}} \text {. }
$$

The elimination of $\beta^{\prime}$ between this and the equation immediately preceding it gives

$$
\frac{4 a c-6 b^{2}}{4 a^{4}}\left(q^{2}-r^{2}\right) q^{2}+\left\{\frac{b}{2 a^{2}}\left(q^{2}-r^{2}\right)+\frac{r^{2}}{\alpha^{\prime}}\right\}^{2}-\frac{q^{2} r^{2}}{\alpha^{\prime 2}}=0 .
$$

Writing in this $4 a c-5 b^{2}=4 M$, we obtain by an easy reduction

$$
4 q^{2} M \alpha^{\prime 2}=r^{2}\left\{2 a^{2}-b \alpha^{\prime}\right\}^{2}
$$

and, taking the square root of each side,

$$
\alpha^{\prime}(2 q \sqrt{ } M+r b)-2 a^{2} r=0 .
$$

A final differentiation gives

$$
\alpha^{\prime}\left(\frac{q M^{\prime}}{\sqrt{ } M}+4 c r\right)+2 a(2 q \sqrt{ } M-5 b r)=0
$$

Finally, eliminating $\alpha^{\prime}$, we obtain

$$
(2 q \sqrt{ } M+r b)(2 q \sqrt{ } M-5 r b)+a r\left(4 c r+\frac{q M^{\prime}}{\sqrt{ } M}\right)=0 .
$$

Hence

$$
4 M q^{2}+q r\left(\frac{a M^{\prime}}{\sqrt{ } M}-8 b \sqrt{ } M\right)+r^{2}\left(4 a c-5 b^{2}\right)=0 ;
$$

or,

$$
4\left(q^{2}+r^{2}\right) M^{\frac{3}{2}}+q r\left(a M^{\prime}-8 b M\right)=0 .
$$

Now

$$
M^{\prime}=\frac{d M}{d x}=\frac{d}{d x}\left(a c-\frac{5 b^{2}}{4}\right)=5 a d-7 b c,
$$

and, consequently,

$$
a M^{\prime}-8 b M=a(5 a d-7 b c)-b\left(8 a c-10 b^{2}\right)=5\left(a^{2} d-3 a b c+2 b^{3}\right)=5 A ;
$$

so that we may write

or,

$$
4\left(q^{2}+r^{2}\right) M^{\frac{3}{2}}=-q r\left(a M^{\prime}-8 b M\right)=-5 q r A ;
$$

where

$$
16\left(q^{2}+r^{2}\right)^{2} M^{3}-25 q^{2} r^{2} A^{2}=0,
$$

$$
3 q^{2}=2-\lambda \text { and }-3 r^{2}=1-2 \lambda \text {. }
$$

Replacing $q^{2}$ and $r^{2}$ by their expressions in terms of $\lambda$, the differential equation becomes

$$
16(\lambda+1)^{2} M^{3}+25\left(2 \lambda^{2}-5 \lambda+2\right) A^{2}=0 .
$$

Some special cases may be noticed. 
When $\lambda=2$ or $\frac{1}{2}$, the equation reduces to $M=0$, which is the differential equation of the common parabola previously obtained.

When $\lambda=3$ or $\frac{1}{3}$, we obtain $256 M^{3}+125 A^{2}=0$ for the equation of the cubical parabola, where the expression on the left-hand side is the QuasiDiscriminant.

When $\lambda=-1$, we find $A=0$ for the differential equation of the general conic.

When $\lambda$ is an imaginary cube root of negative unity, so that $\lambda^{2}-\lambda+1=0$, we have

$$
(\lambda+1)^{2}+\left(2 \lambda^{2}-5 \lambda+2\right)=0
$$

and the differential equation becomes

$$
16 M^{3}-25 A^{2}=0 \text {. }
$$

We shall subsequently avail ourselves of this result in finding the complete primitive of the Halphenian $\Delta$.

In the case where $\lambda$ is infinite, from the complete primitive $\beta=e^{l a}$ we first eliminate the exponential function and afterwards the arbitrary constant $l$.

Thus we find

$$
\beta^{\prime}=l \alpha^{\prime} \beta \text { and } \frac{y_{2}}{\beta^{\prime}}=\frac{y_{2}}{\alpha^{\prime}}+\frac{\beta^{\prime}}{\beta}
$$

or,

$$
y_{2} \beta\left(\alpha^{\prime}-\beta^{\prime}\right)-\alpha^{\prime} \beta^{\prime 2}=0 .
$$

Hence

$$
y_{3} \beta\left(\alpha^{\prime}-\beta^{\prime}\right)-y_{2} \beta^{\prime}\left(\alpha^{\prime}+2 \beta^{\prime}\right)=0 .
$$

The elimination of $\beta$ gives

$$
y_{3} \alpha^{\prime} \beta^{\prime}-y_{2}{ }^{2}\left(\alpha^{\prime}+2 \beta^{\prime}\right)=0
$$

or,

$$
\frac{3 b}{2 a^{2}}=\frac{1}{\beta^{\prime}}+\frac{2}{\alpha^{\prime}} \text {. }
$$

Comparing this with the equation previously obtained,

$$
\frac{b}{2 a^{2}}\left(q^{2}-r^{2}\right)=\frac{q^{2}}{\beta^{\prime}}-\frac{r^{2}}{\alpha^{\prime}}
$$

we see that $q^{2}=1$ and $r^{2}=-2$. Substituting these values in the differential equation

it becomes

$$
\begin{gathered}
16\left(q^{2}+r^{2}\right)^{2} M^{3}-25 q^{2} r^{2} A^{2}=0 \\
8 M^{3}+25 A^{2}=0
\end{gathered}
$$

which is the differential equation corresponding to the complete primitive $\beta=e^{l a}$.

We shall hereafter consider in detail the theory of that special class of pure reciprocants (M. Halphen's Differential Invariants) which retain their form when any homographic substitution is impressed on the variables; that is, when, instead of $x$ and $y$, we write

$$
\frac{l x+m y+n}{l^{\prime \prime} x+m^{\prime \prime} y+n^{\prime \prime}} \text { and } \frac{l^{\prime} x+m^{\prime} y+n^{\prime}}{l^{\prime \prime} x+m^{\prime \prime} y+n^{\prime \prime}} .
$$


Since perspective projection is the geometrical equivalent of homographic substitution, it follows from the definition of Differential Invariants that they are connected with the properties and relations of curves which remain unaffected by perspective projection. For this reason Differential Invariants are sometimes called Projective Reciprocants. Two reciprocants with which we are familiar belong to this important class. One of them, $y_{2}$ or $a$, vanishes at points of inflexion on the curve $y=f(x)$; the other,

$$
9 y_{2}^{2} y_{5}-45 y_{2} y_{3} y_{4}+40 y_{3}^{3} \text {, or } a^{2} d-3 a b c+2 b^{3} \text {, }
$$

which, for reasons given below, we shall call the Mongian, vanishes at sextactic points; that is, at points where a conic can be drawn having 6 -point contact with the given curve.

To illustrate the distinction between a projective and a merely descriptive singularity, consider for an instant the pure reciprocant $4 a c-5 b^{2}$, which, as we have seen, vanishes at all points of a general curve where 5-point contact with a parabola is possible. Now, 5-point contact with a parabola is a descriptive but not a projective singularity; after projection the parabola becomes a general conic, and 5-point contact with it becomes 5-point contact with a general conic, which is not a singularity at all. But inflexions and sextactic points are indelible by projection, and thus belong to the class of projective singularities.

The differential equation to a conic was originally obtained by Monge in the form

$$
9 y_{2}^{2} y_{5}-45 y_{2} y_{3} y_{4}+40 y_{3}^{3}=0
$$

(see Monge, "Sur les Équations différentielles des Courbes du Second Degré," Corresp. sur l'École Polytech., Paris, II. 1809-13, pp. 51-54, and Bulletin de la Soc. Philom., Paris, 1810, pp. 87, 88). At the end of the first chapter of his Differential Equations, Boole mentions this form of equation as due to Monge, but without any reference, and adds the remark: "But here our powers of geometrical interpretation fail, and results such as this can scarcely be otherwise useful than as a registry of integrable forms." The theory of Reciprocants, however, furnishes both a simple interpretation of the Mongian equation and an obvious method of integrating it.

To see that the differential equation of a conic is satisfied at the sextactic points of a given curve, we have only to remember that at such points the derivatives of $y$ with respect to $x$, up to the fifth order, inclusive, are the same for the given curve as for a conic.

We proceed to show how the Mongian may be integrated. Writing in the above equation

$$
y_{2}=2 a, y_{3}=2.3 b, y_{4}=2.3 .4 c, y_{5}=2.3 .4 .5 d,
$$

it becomes

$$
a^{2} d-3 a b c+2 b^{3}=0 \text {, }
$$


where it can hardly fail to be noticed that the left-hand member of the equation is an ordinary Invariant as well as a Reciprocant. It will be proved hereafter that all Differential Invariants possess this double nature.

Now, if $\mu=3 i+w$, where $i$ is the degree and $w$ the weight of any pure reciprocant $R$, the ordinary theory of eduction shows that

is another pure reciprocant.

$$
\frac{d}{d x}\left(\frac{R}{a^{\frac{\mu}{3}}}\right)=\frac{a \frac{d R}{d x}-\mu b R}{a^{\frac{\mu}{3}+1}}
$$

When we consider the letters $a, b, c, \ldots$ in any invariant $I$ to mean $\frac{y_{2}}{2}, \frac{y_{3}}{2.3}, \frac{y_{4}}{2.3 .4}, \ldots$ the parallel theory of generation for Invariants gives the corresponding theorem that if $\nu=3 i+2 w$, where $i$ is the degree and $w$ the weight of $I$,

is also an invariant.

$$
\frac{d}{d x}\left(\frac{I}{a^{\frac{v}{3}}}\right)=\frac{a \frac{d I}{d x}-\nu b I}{a^{\frac{\nu}{3}+1}}
$$

A strict proof of this theorem will subsequently be given. For present purposes it is sufficient to notice the easily verified special cases of the two theorems

and

$$
\frac{d}{d x}\left(\frac{4 a c-5 b^{2}}{a^{\frac{8}{3}}}\right)=\frac{20\left(a^{2} d-3 a b c+2 b^{3}\right)}{a^{\frac{11}{3}}}
$$

$$
\frac{d}{d x}\left(\frac{a c-b^{2}}{a^{\frac{10}{3}}}\right)=\frac{5\left(a^{2} d-3 a b c+2 b^{3}\right)}{a^{\frac{13}{3}}}
$$

It follows as an immediate consequence that the equation

$$
a^{2} d-3 a b c+2 b^{3}=0
$$

admits of the two first integrals

and

$$
a^{-\frac{8}{3}}\left(4 a c-5 b^{2}\right)=\text { const. }
$$

$$
\text { Now, } \quad a^{-\frac{8}{3}}\left(4 a c-5 b^{2}\right)=\frac{d}{d x}\left(a^{-\frac{5}{3}} b\right)=-\frac{1}{2} \frac{d^{2}}{d x^{2}}\left(a^{-\frac{2}{3}}\right)
$$

so that the Mongian equation is equivalent to

$$
\frac{d^{3}}{d x^{3}}\left(a^{-\frac{2}{3}}\right)=0, \text { or to } \frac{d^{3}}{d x^{3}}\left(y_{2}^{-\frac{2}{3}}\right)=0
$$

We thus obtain an integral of the form

$$
y_{2}^{-\frac{2}{3}}=l+2 m x+n x^{2}
$$


from which the complete primitive may be found by two easy integrations. Thus,

gives

$$
y_{1}+p=\int \frac{d x}{\left(l+2 m x+n x^{2}\right)^{\frac{3}{2}}}=\frac{m+n x}{\left(l n-m^{2}\right)\left(l+2 m x+n x^{2}\right)^{\frac{1}{2}}}
$$

which is the equation of a general conic.

By first interchanging the variables $x, y$ in the Mongian equation (whose form remains unaltered by this interchange, since $a^{2} d-3 a b c+2 b^{3}$ is a reciprocant) and then integrating three times with respect to $x$, we should find another integral of the form

$$
x_{2}^{-\frac{2}{3}}=l^{\prime}+2 m^{\prime} y+n^{\prime} y^{2}
$$

The solution may be completed by two integrations, as in the former method.

Mr Hammond remarks that $\frac{2\left(a c-b^{2}\right)}{a^{\frac{10}{3}}}=\frac{d^{2}}{d t^{2}}\left(a^{\frac{2}{3}}\right)$, where $t=y_{1}$. For, since

we have

$$
\begin{gathered}
\frac{d}{d t}=\frac{d x}{d t} \cdot \frac{d}{d x}=\frac{1}{2 a} \cdot \frac{d}{d x} \\
\frac{d}{d t}\left(a^{\frac{2}{3}}\right)=\frac{1}{2 a} \cdot \frac{2}{3} \cdot a^{-\frac{1}{3}} \cdot 3 b=\frac{b}{a^{\frac{4}{3}}}
\end{gathered}
$$

and, consequently,

$$
\frac{d^{2}}{d t^{2}}\left(a^{\frac{2}{3}}\right)=\frac{1}{2 a} \cdot \frac{d}{d x}\left(a^{-\frac{4}{3}} b\right)=2 a^{-\frac{10}{3}}\left(a c-b^{2}\right)
$$

Hence the integral $a^{-\frac{10}{3}}\left(a c-b^{2}\right)=$ const. previously obtained for the Mongian is equivalent to $\frac{d^{2}}{d t^{2}}\left(a^{\frac{2}{3}}\right)=$ constant; that is, to $\frac{d^{2}}{d y_{1}{ }^{2}}\left(y_{2}^{\frac{2}{3}}\right)=$ const. Thus we have another integral of the form

$$
y_{2}{ }^{\frac{2}{3}}=\lambda+2 \mu y_{1}+\nu y_{1}{ }^{2},
$$

from which it is also easy to pass to the complete primitive.

I add a few general remarks relating to the subject-matter of this and the preceding lecture. Instead of the cumbrous terms Projective Reciprocants or Differential Invariants, it may be better to use the single word Principiants to denominate that crowning class or order of Reciprocants which remain, to a factor près, unaltered for any homographic substitutions impressed on the variables. This is the species princeps. If we go back to the species infima, we see the beginning of life in the subject. In general Reciprocants, all that is affirmed is that there exist forms-functions of the derivatives of $y$ in regard to $x$ which (to a factor près) remain unaltered when the variables $x$ and $y$ are interchanged, so that $f\left(y_{1}, y_{2}, y_{3}, \ldots\right)$ becomes 
$\phi\left(x_{1}, x_{2}, x_{3}, \ldots\right)$. The function $\phi$ only differs from $f$ by the acquisition of an extraneous factor $(-)^{\kappa} y_{1}^{\mu}$; that is,

$$
f\left(y_{1}, y_{2}, y_{3}, \ldots\right)=(-)^{\kappa} y_{1}^{\mu} f\left(x_{1}, x_{2}, x_{3}, \ldots\right) .
$$

A particular species of these general (mixed) reciprocants arises when $f\left(y_{1}, y_{2}, y_{3}, \ldots\right)$, differentiated in regard to $y_{1}$, gives a reciprocant. These are Orthogonal Reciprocants, and in them we see the first dawn of free continuous motion as distinguished from mere displacement (or mere interchange of axes). Orthogonal Reciprocants, when $x, y$ are rectangular co-ordinates, remain unaltered (save as to a factor) when the orthogonal axes are moved continuously. A quarter of a revolution of course will reverse their original positions, so that we see the condition of mutual displacement is fulfilled. Thirdly, Reciprocants into whose form the first derivative $y_{1}$ does not enter are called Pure. Their form is invariable when the axes (now taken generally) undergo separate displacement (instead of turning round together) in a plane. Here there is a further development, so to say, of life in the subject.

Finally, in Principiants, a particular species of Pure Reciprocants, the invariance remains good, not merely for any position of the axes of reference, but for any homographic deformation of the plane in which they lie, so that the evanescence of a Principiant corresponds to some property of a curve not only intrinsic but indelible by projection, as, for example, an inflexion, or a double point, or a sextactic point, and so on.

It is clear from this review that the Theory as we have given it goes to the root of the subject, and that the word Reciprocant is rightly chosen as conveying the notion of a property which is common to the entire continuous series of forms bearing that name. All the links of this connected chain are thus comprehended under the general name of Reciprocants.

\section{LECTURE XIV.}

The remaining lectures of the course will be devoted to the theory of Pure and Projective Reciprocants. I shall first treat of the existence and properties of the Protomorphs of Invariants and Reciprocants, using the latter system of protomorphs to obtain all the fundamental forms of Reciprocants in the letters $a, b, c, d, e$. I shall then pass on to the theory of Projective Reciprocants, or Principiants, with its applications contained in M. Halphen's Thèse pour obtenir le grade de docteur ès sciences (Paris, Gauthier-Villars, 1878). It will be seen that M. Halphen's very ingenious methods become greatly simplified when his results are read by the light of an important discovery in the theory of Principiants recently made by myself and Mr Hammond working conjointly, arising out of a theorem put 
forward by one of my hearers. This theorem, on examination, we found was necessarily erroneous and would fail at the very first step of its application. But although the proposition stated was wrong, it contained an Idea which survives and may be incorporated in a valid and extremely important theorem, which I will endeavour to explain.

A Principiant, besides being an Invariant in the original letters $a, b, c, d, \ldots$ is also an Invariant in the letters $a, A, B, C, D, \ldots$ where each capital letter is itself a Reciprocant; and, conversely, every invariant in the capital letters $A, B, C, D, \ldots$ is a Principiant. The invariants in the capital letters form a system of protomorphs for Principiants, so that every Principiant is either some such invariant simply, or a rational integral function of such invariants provided by some power of $a$. Thus, for example, it will be proved that the Cubic Criterium (that is, the Principiant which gives, when equated to zero, the differential equation of a cubic curve) may be expressed as the quotient of

$$
\frac{9}{64} A^{5}+\frac{5}{4} A\left(A^{2} D-3 A B C+2 B^{3}\right)-\left(A C E-A D^{2}-B^{2} E+2 B C D-C^{3}\right)
$$

by the fifth power of $\alpha$.

The proof of this theorem is based upon the fact that we can form a series of terms beginning with the Mongian (namely, $a^{2} d-3 a b c+2 b^{3}$ ), say $A, B, C, D, \ldots$ such that

$$
\begin{aligned}
& \Omega A=0, \\
& \Omega B=A \times \frac{a}{2}, \\
& \Omega C=2 B \times \frac{a}{2}, \\
& \Omega D=3 C \times \frac{a}{2},
\end{aligned}
$$

where

$$
\Omega=a \partial_{b}+2 b \partial_{c}+3 c \partial_{d}+\ldots,
$$

coupled with the fact that every Principiant must be a function of the letters in such series and the small $a$.

Each consequent of the series $A, B, C, D, \ldots$ is, so to say, an Invariant relative to its antecedent; it becomes an actual Invariant when its antecedent vanishes.

In the theorem as originally proposed, each letter of the series was derived by the operation of an eductive generator upon the one which precedes. In the true theorem the scale of relation is between three and not two consecutive terms. Calling the letters $u_{0}, u_{1}, u_{2}, \ldots u_{i}$, we have

$$
(i+7) u_{i+2}-G u_{i+1}+(i+1) M u_{i}=0
$$


where $G$ is the ordinary eductive generator,

$$
4\left(a c-b^{2}\right) \partial_{b}+5(a d-b c) \partial_{c}+6(a e-b d) \partial_{d}+\ldots,
$$

$M$ is the first pure reciprocant after the monomial $a$, namely, $M=a c-\frac{5}{4} b^{2}$, $u_{0}=A=a^{2} d-3 a b c+2 b^{3}$, and $6 u_{1}=G A$.

But although, as I have said, the theorem in the form proposed was absolutely erroneous, its proposer has rendered an invaluable service to the theory by the mere suggestion of what turns out to be true, namely, that every Principiant is an Invariant in regard to a known series of Reciprocants considered as simple elements.

To this theorem there is a correlative one, for it will be shown that there exists a series of invariants $A_{0}, A_{1}, A_{2}, \ldots$, the first term of which, $A_{0}$, is the same as the Mongian $A$, each of the other terms of the series being a Reciprocant relative to the one that precedes it. In fact, we have

$$
\begin{aligned}
& V A_{0}=0, \\
& V A_{1}=-a^{2} A_{0}, \\
& V A_{2}=-2 a^{2} A_{1}, \\
& \cdots \cdots \ldots \ldots \ldots \ldots \ldots . \cdots \\
& V A_{n}=-n a^{2} A_{n-1},
\end{aligned}
$$

where

$$
V=4\left(\frac{a^{2}}{2}\right) \partial_{b}+5 a b \partial_{c}+6\left(a c+\frac{b^{2}}{2}\right) \partial_{d}+\ldots
$$

and, as a consequence, every Principiant will be an Invariant in respect to these Invariants and the first small letter $a$.

Thus, speaking symbolically, we have not only

$$
P=R+I
$$

(a logical equation meaning that $P$ has the same qualities as both $R$ and $I$, or that a Principiant is both a Reciprocant and an Invariant), but also

$$
P=I R \text { and } P=I I,
$$

meaning that a Principiant is an Invariant of Reciprocantive elements, and an Invariant whose elements are themselves Invariants.

I may add that the invariantive elements $A_{0}, A_{1}, A_{2}, A_{3}, \ldots$ are defined by the equations

$$
\begin{aligned}
& A_{0}=A \\
& A_{1}=B-\frac{b}{2} A \\
& A_{2}=C-2\left(\frac{b}{2}\right) B+\left(\frac{b}{2}\right)^{2} A \\
& A_{3}=D-3\left(\frac{b}{2}\right) C+3\left(\frac{b}{2}\right)^{2} B-\left(\frac{b}{2}\right)^{3} A
\end{aligned}
$$

s. IV. 
so that any invariant in the reciprocantive elements $A, B, C, D, \ldots$ is equal to the corresponding invariant in $A_{0}, A_{1}, A_{2}, A_{3}, \ldots$ Thus,

$$
\begin{aligned}
A & =A_{0}, \\
A C-B^{2} & =A_{0} A_{2}-A_{1}^{2} \\
A^{2} D-3 A B C+2 B^{3} & =A_{0}{ }^{2} A_{3}-3 A_{0} A_{1} A_{2}+2 A_{1}{ }^{3} \\
A E-4 B D+3 C^{2} & =A_{0} A_{4}-4 A_{1} A_{3}+3 A_{2}{ }^{2}
\end{aligned}
$$

M. Halphen appears not to have noticed the Principiant $A E-4 B D+3 C^{2}$, which presents itself naturally when the theory is viewed from our present ground of vantage, but $A, A C-B^{2}$ and $A^{2} D-3 A B C+2 B^{3}$ occur in his Thèse in connection with the curve

$$
\alpha=\beta^{\lambda} \gamma^{1-\lambda}
$$

in which $\alpha, \beta, \gamma$ are any linear functions of $x, y, 1$.

When $\lambda=-1$ the differential equation of this curve (the conic $\alpha \beta=\gamma^{2}$ ) is $A=0$, but it is

$$
A C-B^{2}=0
$$

when $\lambda$ is a cube root of negative unity, and

$$
A^{2} D-3 A B C+2 B^{3}=0
$$

when $\lambda$ has an arbitrary value.

Before making out an exhaustive table of all the irreducible forms of pure reciprocants in the letters $a, b, c, d, e$ similar to, but not identical with, the corresponding table for invariants, it seems to me desirable to say something of Protomorphs in general; and this will be better understood if we devote a short space to the protomorphs of Invariants. The simplest forms of these are the following well-known ones of alternately the second and third degrees:

$$
\begin{aligned}
& P_{2}=a c-b^{2} \\
& P_{3}=a^{2} d-3 a b c+2 b^{3} \\
& P_{4}=a e-4 b d+3 c^{2} \\
& P_{5}=a^{2} f-5 a b e+2 a c d+8 b^{2} d-6 b c^{2} \\
& P_{6}=a g-6 b f+15 c e-10 d^{2} \\
& P_{7}=a^{2} h-7 a b g+9 a c f-5 a d e+12 b^{2} f-30 b c e+20 b d^{2}
\end{aligned}
$$

The quadratic Protomorphs $P_{2}, P_{4}, P_{6}, \ldots$, are absolutely unique, for the number of invariants of the type $j ; 2, j$ is $(j ; 2, j)-(j-1 ; 2, j)=1$ if $j$ is even, and $=0$ if $j$ is odd. Their form is so well known that there is no need to dilate upon it here. 
The cubic ones $P_{3}, P_{5}, P_{7}, \ldots$, may be derived from the quadratic ones by means of Cayley's generators, given early in the course, namely,

$$
\begin{aligned}
& P=\left(a c-b^{2}\right) \partial_{b}+(a d-b c) \partial_{c}+(a e-b d) \partial_{d}+\ldots \\
& Q=\left(a c-2 b^{2}\right) \partial_{b}+2(a d-2 b c) \partial_{c}+3(a e-2 b d) \partial_{d}+\ldots
\end{aligned}
$$

Let us first use the $P$ generator

$$
\begin{aligned}
P\left(a c-b^{2}\right) & =a(a d-b c)-2 b\left(a c-b^{2}\right)=a^{2} d-3 a b c+2 b^{3} \\
P\left(a e-4 b d+3 c^{2}\right) & =a(a f-b e)-4 b(a e-b d)+6 c(a d-b c)-4 d\left(a c-b^{2}\right) \\
& =a^{2} f-5 a b e+2 a c d+8 b^{2} d-6 b c^{2} .
\end{aligned}
$$

Similarly, we find

$P\left(a g-6 b f+15 c e-10 d^{2}\right)=a^{2} h-7 a b g+9 a c f-5 a d e+12 b^{2} f-30 b c e+20 b d^{2}$, and so on.

Let $I$ be any invariant whatever of the type $w ; i, j$ (satisfied or unsatisfied); then using the original forms of the generators $P$ and $Q$ as given by Cayley (see Lecture IV), we have

$$
\begin{aligned}
& P I=a\left(b \partial_{a}+c \partial_{b}+d \partial_{c}+\ldots\right) I-i b I, \\
& Q I=a\left(c \partial_{b}+2 d \partial_{c}+3 e \partial_{d}+\ldots\right) I-2 w b I,
\end{aligned}
$$

and, consequently,

$$
(j P-Q) I=a\left\{j b \partial_{a}+(j-1) c \partial_{b}+(j-2) d \partial_{c}+\ldots\right\} I-(i j-2 w) b I .
$$

If in this formula we write

$$
O=j b \partial_{a}+(j-1) c \partial_{b}+(j-2) d \partial_{c}+\ldots,
$$

it becomes

$$
(j P-Q) I=a O I-(i j-2 w) b I \text {, }
$$

which, when $I$ is a satisfied invariant, so that $i j-2 w=0$ and $O I=0$, reduces to

$$
(j P-Q) I=0 \text {, }
$$

showing that the forms obtained by operating with either $P$ or $Q$ on any satisfied invariant are the same to a numerical factor près.

Now, each quadratic protomorph is a satisfied invariant (for when $w=j$ and $i=2, \quad i j-2 w=0$ ), and therefore the cubic protomorphs found by operating on the quadratic ones with $Q$ will only differ by a numerical factor from those already obtained by the operation of $P$. But we must not conclude from this that the cubic protomorphs are unique. Their number is in fact given by the formula

$$
(j ; 3, j)-(j-1 ; 3, j) \text {, }
$$

where it is obvious that

$$
(j-1 ; 3, j)=(j-1 ; 3, j-1) ;
$$

so that the above formula may be written

$$
(j ; 3, j)-(j-1 ; 3, j-1) \text {, or say } \Delta(j ; 3, j) \text {. }
$$


Now, there is a simple rule for finding $(j ; 3, j)$; it is the nearest integer to $\frac{(j+3)^{2}}{12}$. From the following table, obtained by the use of this rule,

$$
\begin{array}{rl|l|l|l|l|l|c|c|c|c|c|c|c|c|}
j= & 2 & 3 & 4 & 5 & 6 & 7 & 8 & 9 & 10 & 11 & 12 & 13 & 14 & 15 \\
(j ; 3, j)= & 2 & 3 & 4 & 5 & 7 & 8 & 10 & 12 & 14 & 16 & 19 & 21 & 24 & 27 \\
\Delta(j ; 3, j)= & 1 & & 1 & & 1 & & 2 & & 2 & & 2 & & 3
\end{array}
$$

it may be seen that for any odd number $j=>9$ there are two or more forms of extent $j$ equally entitled to rank as protomorphs. If $l$ be the last letter which occurs in one of these forms, its first term will of course be $a^{2} l$; the difference between any two such forms will not involve the letter $l$, and will only extend to $k$, but will still be of the same (potential) extent as $l$.

The property of the protomorphs $a, P_{2}, P_{3}, P_{4}, \ldots$ is that every invariant is a rational integral function of them divided by some power of $a$, as appears from the fact that $Q$, any given rational integral function whatever of the letters $a, b, c, d, e, \ldots$, may obviously be expressed as a rational integral function of $a, b, P_{2}, P_{3}, P_{4}, \ldots$ divided by some power of $a$. Thus,

$$
Q=a^{-m} \phi\left(a, b, P_{2}, P_{3}, P_{4}, \ldots\right) \text {. }
$$

Suppose $Q$ to be an invariant $I$; then

and, consequently,

$$
I a^{m}=\phi\left(a, b, P_{2}, P_{3}, P_{4}, \ldots\right)
$$

$$
\Omega\left(I a^{m}\right)=\frac{d \phi}{d a} \Omega a+\frac{d \phi}{d b} \Omega b+\frac{d \phi}{d P_{2}} \Omega P_{2}+\frac{d \phi}{d P_{3}} \Omega P_{3}+\ldots,
$$

where $\Omega$ is the annihilator for invariants; so that

$$
\Omega\left(I a^{m}\right)=0, \Omega a=0, \Omega P_{2}=0, \Omega P_{3}=0, \ldots .
$$

We have therefore

$$
\frac{d \phi}{d b} \Omega b=a \frac{d \phi}{d b}=0
$$

Hence $\phi$ does not contain $b$, but is a rational integral function of the protomorphs alone, and

$$
I=a^{-m} \phi\left(a, P_{2}, P_{3}, P_{4}, \ldots\right) .
$$

I shall show how to obtain a similar scale of forms possessing like properties for pure reciprocants.

\section{LECTURE XV.}

A Protomorph may be defined as a form whose weight is equal to its actual extent, so that its type is $j ; i, j$. The first protomorph is $a$, which corresponds to $j=0$. For higher values of $j$ it follows immediately from the definition that every protomorph will contain a term $a^{i-1} l$, in which the letter of highest extent appears only in the first degree multiplied by a 
power of the first letter. The existence of this term enables us to instantly recognize a protomorph. As in the case of invariants, it will be shown that every pure reciprocant is either a rational integral function of protomorphs or else such a function divided by some power of $a$. But first it will be better to prove $a$ priori their existence and exhibit examples of them for the earlier values of $j$.

It was proved, in Lecture IX, that the number of pure reciprocants of the type $w ; i, j$ is at least equal to

$$
(w ; i, j)-(w-1 ; i+1, j)
$$

Now, obviously, the number of partitions of $w$ into $i$ parts not exceeding $w+\epsilon$ is the same as the number of partitions of $w$ into $i$ parts not exceeding $w$, so that

$$
(w ; i, w+\epsilon)=(w ; i, w)
$$

and since, by a well-known theorem, $(w ; i, j)=(w ; j, i)$, we see that

$$
(w ; w+\epsilon, j)=(w ; j, w+\epsilon)=(w ; j, w)=(w ; w, j)
$$

a result which follows more immediately from the consideration that the partitions of $w ; w+\epsilon, j$ differ only from those of $w ; w, j$ by $\epsilon$ columns of zeros, as we see in the annexed example:

\begin{tabular}{l|l}
$\frac{3 ; 5,3}{30000}$ & $3 ; 3,3$ \\
\cline { 1 - 2 } 21000 & 210 \\
11100 & 111
\end{tabular}

Hence, if $w=j$, and $i=>j$, we have

$$
(w ; i, j)=(j ; j, j)
$$

and

$$
(w-1 ; i+1, j)=(j-1 ; j-1, j-1) .
$$

Thus, the number of pure reciprocants of the type $j ; j, j$ is

$$
(j ; j, j)-(j-1 ; j-1, j-1)
$$

in other words, the difference between the indefinite partitions of $j$ and those of $j-1$. Expressed by means of generating functions, this difference is the coefficient of $x^{j}$ in

$$
\frac{1-x}{(1-x)\left(1-x^{2}\right)\left(1-x^{3}\right) \ldots\left(1-x^{j}\right)}
$$

$=$ coefficient of $x^{j}$ in the expansion of

$$
\frac{1}{\left(1-x^{2}\right)\left(1-x^{3}\right) \ldots\left(1-x^{j}\right)}
$$

This coefficient is a positive integer for all values of $j$ (except $j=1$, when it is zero), which proves the existence of reciprocants of the type $j ; j, j$ when $j$ has any value except unity.

But we wish to prove the existence of one or more reciprocants of the type $j ; j, j$ which actually contain a term of the form $a^{j-1} l$, where the letter $l$ 
is of extent $j$. The number of such forms is the difference between the number of pure reciprocants of the types $j ; j, j$ and $j ; j, j-1$.

Now, the number of linearly independent pure reciprocants of the type $j ; j, j$ has just been shown to be

$$
(j ; j, j)-(j-1 ; j-1, j-1) \text {. }
$$

And, in like manner, that of the linearly independent reciprocants of the type $j ; j, j-1$ is

$$
\begin{aligned}
& (j ; j, j-1)-(j-1 ; j+1, j-1) \\
= & (j ; j, j-1)-(j-1 ; j-1, j-1) .
\end{aligned}
$$

The difference between these two numbers is therefore

$$
(j ; j, j)-(j ; j, j-1)=1 .
$$

For the only partition not common to the two types is $j \cdot 0^{j-1}$, made up of one $j$ and $j-1$ zeros, which belongs to the first type, but not to the second. Hence reciprocants of the type $j ; j, j$ contain one term which those of the type $j ; j, j-1$ do not, and which can only be $a^{j-1} l$. This proves the existence of protomorphs.

In the latter part of the above proof we have assumed the truth of the theorem, which, however probable, is not demonstrated, that the number of reciprocants of the type $w ; i, j$ is $(w ; i, j)-(w-1 ; i+1, j)$ and no more [that concerns the subtrahend, namely, $(j ; j, j-1)-(j-1 ; j-1, j-1)$ ].

We shall, however, have an independent method of arriving at Protomorphs by direct generation, just as we saw that all the cubic protomorphs to invariants were derivable by direct operation of generators from the quadratic ones.

The difference between the two cases is that the lowest degree of Invariantive Protomorphs fluctuates alternately between 2 and 3. For Reciprocantive Protomorphs the lowest degree corresponding to a given extent fluctuates, but has a tendency to rise, and goes on progressing until it exceeds any assignable number.

It is interesting to find what the degrees are for successive values of $j$. The calculations required are greatly facilitated by an extensive table of partitions given by Euler in 1750, and partly reproduced by Cayley in the American Journal of Mathematics, Vol. IV., Part III. In the table as presented by Cayley, the number in column $j$ and line $i$ means the number of ways of partitioning $j$ into exactly $i$ parts (zeros excluded). Hence, to find the number of ways of partitioning $j$ into $i$ parts or fewer, that is, to find $(j ; i, \infty)$ or its equivalent $(j ; i, j)$, we must add up the numbers in the 1st, 2 nd, $3 \mathrm{rd}, \ldots i$ th lines of column $j$. 
When these summations are made we obtain the subjoined table:

Extent $j=$

\begin{tabular}{|c|c|c|c|c|c|c|c|c|c|c|c|c|c|c|c|c|c|c|c|}
\hline & 0 & 1 & 2 & 3 & 4 & 5 & 6 & 7 & 8 & 9 & 10 & 11 & 12 & 13 & 14 & 15 & 16 & 17 & 18 \\
\hline 1 & 1 & 1 & 1 & 1 & 1 & 1 & 1 & 1 & 1 & 1 & 1 & 1 & 1 & 1 & 1 & 1 & 1 & 1 & 1 \\
\hline 2 & 1 & 1 & 2 & 2 & 3 & 3 & 4 & 4 & 5 & 5 & 6 & 6 & 7 & 7 & 8 & 8 & 9 & 9 & 10 \\
\hline 3 & 1 & 1 & 2 & 3 & 4 & 5 & 7 & 8 & 10 & 12 & 14 & 16 & 19 & 21 & 24 & 27 & 30 & 33 & 37 \\
\hline 4 & 1 & 1 & 2 & 3 & 5 & 6 & 9 & 11 & 15 & 18 & 23 & 27 & 34 & 39 & 47 & 54 & 64 & 72 & 84 \\
\hline 5 & 1 & 1 & 2 & 3 & 5 & 7 & 10 & 13 & 18 & 23 & 30 & 37 & 47 & 57 & 70 & 84 & 101 & 119 & 141 \\
\hline 6 & 1 & 1 & 2 & 3 & 5 & 7 & 11 & 14 & 20 & 26 & 35 & 44 & 58 & 71 & 90 & 110 & 136 & 163 & 199 \\
\hline 7 & 1 & 1 & 2 & 3 & 5 & 7 & 11 & 15 & 21 & 28 & 38 & 49 & 65 & 82 & 105 & 131 & 164 & 201 & 248 \\
\hline 8 & 1 & 1 & 2 & 3 & 5 & 7 & 11 & 15 & 22 & 29 & 40 & 52 & 70 & 89 & 116 & 146 & 186 & 230 & 288 \\
\hline
\end{tabular}

The number of pure reciprocants of the type $j ; i, j$ is

$$
(j ; i, j)-(j-1 ; i+1, j)=(j ; i, j)-(j-1 ; i+1, j-1) .
$$

To find the minimum degree for protomorphs of extent $j$ we have therefore only to see for what value of $i$ any figure in the $j$ column first becomes greater than the figure in the column to the left one place lower down. The fluctuations of the minimum degree are indicated by the dark irregularly waving line which runs through the table.

Accordingly, we find that the types of the protomorphs, omitting $w$, which is always equal to $j$, are as follows:

$(2,2),(3,3),(3,4),(4,5),(3,6),(4,7),(4,8),(5,9),(5,10),(5,11),(5,12), \ldots$, whereas for invariants they are

$(2,2),(3,3),(2,4),(3,5),(2,6),(3,7),(2,8),(3,9),(2,10),(3,11),(2,12), \ldots$

Corresponding to the extents

$$
2,3,4,5,6,7,8,9,10,11,12, \ldots \text {, }
$$

the lowest degrees of the Reciprocantive Protomorphs are

$$
2,3,3,4,3,4,4,5,5,5,5, \ldots .
$$

Contrast this with the regularly fluctuating series

$$
2,3,2,3,2,3,2,3,2,3,2,3, \ldots,
$$

which shows the minimum degrees of invariantive protomorphs for successive extents.

It may be proved, from known formulae in the theory of partitions, that as the extent increases the minimum degree of reciprocantive protomorphs increases (on the whole) and ultimately becomes infinite when the extent is so. 
The apparent number of protomorphs to the several types is $(2,2),(3,3),(3,4),(4,5),(3,6),(4,7),(4,8),(5,9),(5,10),(5,11),(5,12), \ldots$
1
1
1
11
1
12
$2 \quad 3$
$4 \quad 2$
3

The explanation of this multiplicity is the same as that previously given for the case of invariants : the difference between any two protomorphs of a given type $j ; i, j$ will be a reciprocant (no longer a protomorph) of the type $j ; i, j-1$.

For the only term containing the letter $l$ (of extent $j$ ) will disappear from the result of subtraction; and, accordingly, the above numbers, each diminished by unity, will give the numbers of a set of reciprocants of the same degree-weight as the protomorphs, but of a smaller (actual) extent.

Assuming that the number of pure reciprocants of the type $w ; i, j$ is correctly given by the formula

$$
(w ; i, j)-(w-1 ; i+1, j)
$$

Euler's great table of partitions, already referred to, enables us to carry on the determination of the minimum degree and multiplicity of protomorphs for all extents as far as 59 .

If $m$ is the multiplicity corresponding to the minimum degree $i$ of a reciprocantive protomorph whose extent is $j$, we form without difficulty, using only the principles explained above, the following table:

\begin{tabular}{|c|c|c|c|c|c|c|c|c|c|c|c|c|}
\hline$j=$ & 0 & 1 & 2 & 3 & 4 & 5 & 6 & 7 & 8 & 9 & 10 & 11 \\
\hline$i=$ & 1 & - & 2 & 3 & 3 & 4 & 3 & 4 & 4 & 5 & 5 & $\check{5}$ \\
\hline$=$ & 1 & 0 & 1 & 1 & 1 & 1 & 1 & 1 & 2 & 3 & 4 & 2 \\
\hline & 12 & 13 & 14 & 15 & 16 & 17 & 18 & 19 & 20 & 21 & 22 & 23 \\
\hline$i=$ & 5 & 6 & 6 & 6 & 6 & 7 & 7 & 7 & 7 & 7 & 8 & 8 \\
\hline$=$ & 3 & 6 & 8 & 5 & 5 & 15 & 18 & 12 & 12 & 2 & 40 & 32 \\
\hline$=$ & 24 & 25 & 26 & 27 & 28 & 29 & 30 & 31 & 32 & 33 & 34 & 35 \\
\hline$i=$ & 8 & 8 & 8 & 9 & 9 & 9 & 9 & 10 & 10 & 10 & 10 & 10 \\
\hline$m=$ & 32 & 14 & 6 & 84 & 82 & 58 & 4.5 & 207 & 211 & 180 & 161 & 102 \\
\hline$=$ & 36 & 37 & 38 & 39 & 40 & 41 & 42 & 43 & 44 & 45 & 46 & 47 \\
\hline$i=$ & 10 & 11 & 11 & 11 & 11 & 11 & 11 & 12 & 12 & 12 & 12 & 12 \\
\hline$m=$ & 45 & 482 & 469 & 391 & 320 & 167 & 13 & $\mid 1126$ & 1064 & 881 & 687 & $337 \mid$ \\
\hline$J-$ & 48 & 49 & 50 & 51 & 52 & 53 & 54 & 55 & 56 & รั7 & 58 & 59 \\
\hline 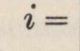 & 13 & 13 & 13 & 13 & 13 & 13 & 13 & 14 & 14 & 14 & 14 & 14 \\
\hline$n=$ & 2829 & 2666 & 2492 & 2097 & 1643 & 892 & 26 & 6394 & 6017 & 5227 & $4: 266$ & $|2755|$ \\
\hline
\end{tabular}

Notice the repetitions of $i$ indicated by the series $1^{1}, 0^{1}, 2^{1}, 3^{2}, 4^{1}, 3^{1}, 4^{2}, 5^{4}, 6^{4}, 7^{5}, 8^{5}, 9^{4}, 10^{6}, 11^{6}, 12^{5}, 13^{7}, 14^{5+?}$. 
It will be observed that there is a general tendency of the number of equal values of $i$ to increase, but that this is subject to occasional fluctuations. When $j=5, i=4$; but when $j=6, i=3$, so that the minimum value of $i$ recedes. After this point is reached, $i$ either advances or remains stationary, but never recedes.

In order actually to find the protomorphs, we may use the annihilator $V$. This was my original method of obtaining them; a shorter way, analogous to that used by Halphen for differential invariants (principiants), has been previously mentioned, but it will be instructive to begin with the method of indeterminate coefficients. In the first place we have the form $a$ of weight 0 , which is annihilated by

$$
V=2 a^{2} \partial_{b}+5 a b \partial_{c}+\left(6 a c+3 b^{2}\right) \partial_{d}+(7 a d+7 b c) \partial_{e}+\ldots
$$

For weight 1 there is no pure reciprocant. We could not make $R=\lambda \alpha^{i-1} b$, for then $V R=2 \lambda a^{i+1}$, which cannot vanish unless $\lambda=0$ and consequently $R=0$.

To find the Protomorph of extent 2 , assume $R=\lambda a c+\mu b^{2}$; then

$$
V R=4 \mu a^{2} b+5 \lambda a^{2} b=(4 \mu+5 \lambda) a^{2} b .
$$

Hence $\lambda$ and $\mu$ are proportional to 4 and -5 , and we may write

$$
R=4 a c-5 b^{2} \text {. }
$$

For extent 3, assuming $R=\lambda a^{2} d+\mu a b c+\nu b^{3}$, we have

$$
V R=2 \mu a^{3} c+6 \nu a^{2} b^{2}+5 \mu a^{2} b^{2}+6 \lambda a^{3} c+3 \lambda a^{2} b^{2},
$$

which vanishes when

$$
2 \mu+6 \lambda=0,6 \nu+5 \mu+3 \lambda=0 \text {. }
$$

We may therefore write $\lambda=1, \mu=-3, \nu=2$, and thus obtain

$$
R=a^{2} d-3 a b c+2 b^{3} \text {. }
$$

For extent 4 the table of minimum degrees indicates the existence of a protomorph of degree 3. To find its value we assume

$$
R=\kappa a^{2} e+\lambda a b d+\mu a c^{2}+\nu b^{2} c .
$$

Operating with $V$, we find

$$
V R=\begin{array}{ccc}
a^{3} d & a^{2} b c & a b^{3} \\
2 \lambda & 4 \nu & . \\
\cdot & 10 \mu & 5 \nu \\
\cdot & 6 \lambda & 3 \lambda \\
7 \kappa & 7 \kappa & .
\end{array}
$$

In order that $V R$ may vanish, we must have

$$
2 \lambda+7 \kappa=0,4 \nu+10 \mu+6 \lambda+7 \kappa=0, \text { and } 5 \nu+3 \lambda=0 .
$$

To avoid fractions, let $\kappa=50$; then $\lambda=-175, \nu=105$, and $\mu=28$; thus,

$$
R=50 a^{2} e-175 a b d+28 a c^{2}+105 b^{2} c ;
$$

whereas, the protomorph of extent 4 for Invariants is $a e-4 b d+3 c^{2}$. There is no reciprocant of degree 2 weight 4 to correspond to this. 


\section{LECTURE XVI.}

By using the generator for pure reciprocants instead of the annihilator $V$, we readily obtain the protomorph of extent 5 and of the fourth degree whose existence is indicated in the previously given table of minimum degrees. We have only to operate on the protomorph of degree 3 and extent 4 with

$$
G=4\left(a c-b^{2}\right) \partial_{b}+5(a d-b c) \partial_{c}+6(a e-b d) \partial_{d}+7(a f-b e) \partial_{e}+\ldots
$$

Thus,

$$
\begin{aligned}
& G\left(50 a^{2} e-175 a b d+28 a c^{2}+105 b^{2} c\right) \\
& \quad=4\left(a c-b^{2}\right)(-175 a d+210 b c) \\
& \quad+5(a d-b c)\left(56 a c+105 b^{2}\right) \\
& \quad+6(a e-b d)(-175 a b) \\
& \quad+7(a f-b e)\left(50 a^{2}\right) .
\end{aligned}
$$

Rejecting the numerical factor 35 , which is common to all the terms in the result, and at the same time writing the terms themselves in reverse order, we have

$$
\begin{gathered}
10 a^{2}(a f-b e)-30 a b(a e-b d)+(a d-b c)\left(8 a c+15 b^{2}\right)+4\left(a c-b^{2}\right)(-5 a d+6 b c) \\
=10 a^{3} f-40 a^{2} b e-12 a^{2} c d+65 a b^{2} d+16 a b c^{2}-39 b^{3} c,
\end{gathered}
$$

which is the protomorph in question.

The form just found is irreducible, as indeed it ought to be, since the minimum degree for extent 5 is greater than that for extent 4 by unity, which exactly corresponds with the unit increase of degree due to the operation of $G$. But if we use $G$ to generate a protomorph of extent 4 from that of extent 3 , the resulting form will be reducible. In fact

$$
\begin{aligned}
G & \left(a^{2} d-3 a b c+2 b^{3}\right) \\
& =4\left(a c-b^{2}\right)\left(-3 a c+6 b^{2}\right)+5(a d-b c)(-3 a b)+6(a e-b d) a^{2} \\
& =3\left(2 a^{3} e-7 a^{2} b d-4 a^{2} c^{2}+17 a b^{2} c-8 b^{4}\right) .
\end{aligned}
$$

If now we write

$$
\begin{gathered}
a c-\frac{5}{4} b^{2}=M \\
a^{2} d-3 a b c+2 b^{3}=A \\
a^{3} e-\frac{7}{2} a^{2} b d-2 a^{2} c^{2}+\frac{17}{2} a b^{2} c-4 b^{4}=B
\end{gathered}
$$

we have shown that

$$
G A=6 B \text {. }
$$

But

$$
\begin{aligned}
50 B+128 M^{2} & =25\left(2 a^{3} e-7 a^{2} b d-4 a^{2} c^{2}+17 a b^{2} c-8 b^{4}\right)+8\left(4 a c-5 b^{2}\right)^{2} \\
& =a\left(50 a^{2} e-175 a b d+28 a c^{2}+105 b^{2} c\right)
\end{aligned}
$$

so that $B$ is reducible, being expressible as a rational integral function of $a, M$, and the previously obtained protomorph of degree 3 and extent 4 . 
The general theory of the generator $G$ is contained in that of the differentiation of absolute reciprocants, in which, if $\mu=3 i+w$, where $w$ is the weight and $i$ the degree of any pure reciprocant $R$, we have

and, consequently,

$$
\frac{R}{a^{\frac{\mu}{3}}}= \pm \frac{R_{1}}{a_{1}^{\frac{\mu}{3}}}
$$

$$
\frac{d}{d x}\left(\frac{R}{a^{\frac{\mu}{3}}}\right)= \pm \frac{d y}{d x} \cdot \frac{d}{d y}\left(\frac{R_{1}}{a_{1}^{\frac{\mu}{3}}}\right)
$$

where $R_{1}$ and $a_{1}$ are what $R$ and $a$ become when $x$ and $y$ are interchanged. Hence

$$
\frac{a \frac{d R}{d x}-\frac{\mu}{3} R \frac{d a}{d x}}{a^{\frac{\mu}{3}+1}}
$$

and therefore also the numerator of this fraction is a reciprocant.

Remembering that

$$
\frac{d a}{d x}=3 b, \frac{d b}{d x}=4 c, \frac{d c}{d x}=5 d, \ldots
$$

the numerator may be written

$$
a \frac{d R}{d x}-\mu b R=G R
$$

The ordinary expression for $G$ is found by writing

$$
\begin{aligned}
& a \frac{d}{d x}-\mu b=a\left(3 b \partial_{a}+4 c \partial_{b}+5 d \partial_{c}+\ldots\right) \\
&-b\left(3 a \partial_{a}+4 b \partial_{b}+5 c \partial_{c}+\ldots\right)
\end{aligned}
$$

If the actual extent of $R$ is $j$, that of $G R$ is $j+1$; for the operation of $G$ introduces an additional letter. Both the weight and degree are also increased by unity. Thus, the type of $R$ being $w ; i, j$, that of $G R$ is

$$
w+1 ; i+1, j+1 \text {. }
$$

Suppose the weight of $R$ to be equal to its actual extent; then $R$ is a protomorph of the type $j ; i, j$, and $G R$, whose type is $j+1 ; i+1, j+1$, is also a protomorph. This proves the existence of protomorphs for every possible extent. Starting with the form $4 a c-5 b^{2}$ we obtain, by successive eduction, a series of protomorphs of the type $j ; j, j$ for which the general expression is

$$
G^{j-2}\left(4 a c-5 b^{2}\right)
$$

where $j$ has any of the values $2,3,4, \ldots$.

If $R$ is a protomorph of minimum degree, $G R$ (if irreducible) will also be a protomorph of minimum degree. Hence the minimum degree can never increase by more than one unit when the extent is increased by unity. 
The second educt $G^{2} R$ is always reducible; for

$$
\begin{aligned}
G^{2} R & =\left\{a \frac{d}{d x}-(\mu+4) b\right\}\left(a \frac{d}{d x}-\mu b\right) R \\
& =\left\{a^{2} \frac{d^{2}}{d x^{2}}-(2 \mu+1) a b \frac{d}{d x}-4 \mu a c+\mu(\mu+4) b^{2}\right\} R .
\end{aligned}
$$

Combining this with $M=a c-\frac{5}{4} b^{2}$, we have

$$
5 G^{2} R+4 \mu(\mu+4) M R=a\left\{5 a \frac{d^{2}}{d x^{2}}-5(2 \mu+1) b \frac{d}{d x}+4 \mu(\mu-1) c\right\} R,
$$

where the right-hand side is divisible by $a$, showing that the degree of $G^{2} R$ is always depressible by unity. $R$ being a protomorph of degree $i$ and extent $j$,

$$
\left\{5 a \frac{d^{2}}{d x^{2}}-5(2 \mu+1) b \frac{d}{d x}+4 \mu(\mu-1) c\right\} R
$$

is one of degree $i+1$ and extent $j+2$. Hence we may conclude that an increase in the minimum degree for protomorphs cannot be immediately followed by another increase; for, if this were possible, the minimum degree for extent $j+2$ would be $i+2$, instead of being $i+1$ at most.

This conclusion is in accordance with the sequence of the values of $i$ in the table of minimum degrees, and as far as it goes confirms the exactitude of the formula $(w ; i, j)-(w-1 ; i+1, j)$ for the number of pure reciprocants which was assumed in calculating the table.

The method previously employed to prove that every invariant is a rational integral function of protomorphs, or such function divided by a power of $a$, may be very easily extended to the case of reciprocants.

In the first place, it is obvious that every rational integral function of the letters $a, b, c, d, \ldots$ is by successive substitutions reducible to the form

$$
a^{-\theta} \Phi\left(a, b, P_{2}, P_{3}, P_{4}, \ldots P_{j}\right)
$$

where $P_{j}$ means the protomorph of extent $j$.

Let any reciprocant $R$ be put under this form; then

and, consequently,

$$
a^{\theta} R=\Phi\left(a, b, P_{2}, P_{3}, P_{4}, \ldots P_{j}\right),
$$

$$
V\left(a^{\theta} R\right)=\frac{d \Phi}{d a} V a+\frac{d \Phi}{d b} V b+\frac{d \Phi}{d P_{2}} V P_{2}+\ldots+\frac{d \Phi}{d P_{j}} V P_{j}
$$

Now, $V$ annihilates $R, a, P_{2}, P_{3}, \ldots P_{j}$, since these are all pure reciprocants. Hence the above identity reduces to $\frac{d \Phi}{d b} V b=0$, from which (since $V b$ does not vanish) we conclude that $\Phi$ does not contain $b$ explicitly. Thus,

$$
a^{\theta} R=\Phi\left(a, P_{2}, P_{3}, P_{4}, \ldots P_{j}\right),
$$

and the theorem is established for reciprocants. 
The Protomorphs for Reciprocants as far as extent 8 are as follows:

$$
\begin{aligned}
& P_{2}=4 a c-5 b^{2}, \\
& P_{3}=a^{2} d-3 a b c+2 b^{3}, \\
& P_{4}=50 a^{2} e-175 a b d+28 a c^{2}+105 b^{2} c \\
& P_{5}=10 a^{3} f-40 a^{2} b e-12 a^{2} c d+65 a b^{2} d+16 a b c^{2}-39 b^{3} c, \\
& P_{6}= 14 a^{2} g-63 a b f-1350 a c e+1782 b^{2} e+1470 a d^{2}-4158 b c d+2310 c^{3}, \\
& P_{7}=7 a^{3} h-35 a^{2} b g-539 a^{2} c f+735 a b^{2} f+605 a^{2} d e+306 a b c e-1485 b^{3} e \quad-2135 a b d^{2}+1001 a c^{2} d+3465 b^{2} c d-1925 b c^{3}, \\
& P_{8}=420 a^{3} i-2310 a^{2} b h-25648 a^{2} c g+9240 a^{2} d f+21780 a^{2} e^{2}+36680 a b^{2} g \\
&+ \\
& \quad+85386 a b c f-191730 a b d e-59220 a c^{2} e+120540 a c d^{2} \\
& \quad \\
& \quad+126945 b^{3} f+252126 b^{2} c e+169260 b^{2} d^{2}-419034 b c^{2} d
\end{aligned}
$$

The work necessary for obtaining the first four of these, $P_{2}, P_{3}, P_{4}, P_{5}$, has been fully set out. Since $P_{4}$ is of degree 3 , its second educt, $G^{2} P_{4}$, is of degree $\check{5}$ and its reduced second educt of degree 4 . A linear combination of this with a form whose leading term is $a^{2} c e$ becomes divisible by $a$ and gives $P_{6}$; but as this requires the preliminary calculation of the form $\left(a^{2} c e\right)$, it is simpler to find $P_{6}$ directly by the method of indeterminate coefficients, and thence by eduction to get $P_{7}$ and $P_{8}$. Thus (to a numerical factor près) $P_{7}$ is the educt and $P_{8}$ the reduced second educt of $P_{6}$. Beyond this point the calculation of protomorphs has not at present been carried.

Referring to the table which gives the minimum degree and multiplicity for a Protomorph of any extent, we see that the multiplicity exceeds unity when the extent $j=>8$, and is exactly equal to 2 when $j=8,11$, or 21 .

Hence the protomorphs as far as $P_{7}$ inclusive are unique; but there are two forms of extent 8 and degree 4, any linear combination of which (provided it contains the term $a^{3} i$ ) may be regarded as a protomorph. One of these forms is $P_{8}$, whose value is given above; the other is a linear combination of $P_{8}$ with a form, whose leading term is $a^{2} c g$, hereafter to be set forth.

The irreducible forms for extent 2 are $a$ and $P_{2}$; every other form must be simply a power of $P_{2}$ multiplied by a power of $a$. We proceed to the calculation of all the Irreducible Forms for the extents 3 and 4 respectively. When $j=3$, we may combine the protomorphs

and

$$
4 a c-5 b^{2}
$$

with one another.

$$
a^{2} d-3 a b c+2 b^{3}
$$

Adding 125 times the square of the latter to 4 times the cube of the former and dividing by $a$, there results the form

$$
125 a^{3} d^{2}-750 a^{2} b c d+500 b^{3} d+256 a^{2} c^{3}+165 a b^{2} c^{2}-300 b^{4} c .
$$


This form is analogous to the discriminant of the cubic, but is of a higher degree by one unit. Its type is $6 ; 5,3$, whereas that of the discriminant is $6 ; 4,3$.

In the case of invariants, we have to combine $a c-b^{2}$ with $a^{2} d-3 a b c+2 b^{3}$. The square of the second, added to 4 times the cube of the first, gives $a^{4} d^{2}-6 a^{3} b c d+4 a^{2} b^{3} d+9 a^{2} b^{2} c^{2}-12 a b^{4} c+4 b^{6}+4 a^{3} c^{3}-12 a^{2} b^{2} c^{2}+12 a b^{4} c-4 b^{6}$.

Here the term $12 a b^{4} c$ is nullified by $-12 a b^{4} c$, so that the result contains $a^{2}$, the other factor being the discriminant

$$
a^{2} d^{2}-6 a b c d+4 b^{3} d+4 a c^{3}-3 b^{2} c^{2}
$$

which is of the type $6 ; 4,3$.

We may show à priori, assuming the problematical but highly probable formula $(w ; i, j)-(w-1 ; i+1, j)$, that the type $6 ; 4,3$ does not belong to any reciprocant.

For, as seen in the partitionments set out below,

$$
\begin{array}{ll}
(6 ; 4,3)-(5 ; 5,3)=5-5=0 \\
3.3 & 3.2 \\
3.2 .1 & 3.1 .1 \\
3.1 .1 .1 & 2.2 .1 \\
2.2 .2 & 2.1 .1 .1 \\
2.2 .1 .1 & 1.1 .1 .1 .1
\end{array}
$$

We can by no other means combine the protomorphs with one another or with the Quasi-Discriminant $\left(125 a^{3} d^{2} \ldots\right)$ so as to obtain additional fundamental forms. Every Rational Integral Pure Reciprocant of extent 3 is therefore necessarily a rational integral function of the four forms deg. wt.

1.0 a,

$2.24 M=4 a c-5 b^{2}$,

$3.3 A=a^{2} d-3 a b c+2 b^{3}$,

5. $6 \quad\left(a^{3} d^{2}\right)=125 a^{3} d^{2}-750 a^{2} b c d+500 b^{3} d+256 a^{2} c^{3}+165 a b^{2} c^{2}-300 b^{4} c$.

These are connected by a syzygy of degree-weight 6.6 , namely

$$
125 A^{2}+256 M^{3}=a\left(a^{3} d^{2}\right)
$$

analogous to the syzygy of the same degree-weight, in the Theory of the Binary Cubic, which connects the Discriminant with $a$ and the Protomorphs of extent 2 and 3.

It will be clearly seen from an inspection of the fundamental forms that there is no law for the coefficients of Reciprocants akin to that of their algebraical sum being zero in Invariants. 


\section{LECTURE XVII.}

The fundamental reciprocants for extent 3 , given in the last lecture, agree with the irreducible invariants of a binary cubic both in number and type, with the single exception that the degree of the cubic discriminant is lower by unity than that of the reciprocant corresponding to it. When the extent is raised to 4 , both the discriminant and its analogue cease to rank among the irreducible forms, the former being expressible as a rational integral function of invariants of lower degree, and the latter as a similar function of reciprocants. But the increase of extent introduces three additional reciprocants whose leading terms are $a^{2} e, a^{2} c e$ and $a^{3} e^{2}$, whereas the additional invariants are only two in number and begin with ae and ace respectively.

The irreducible reciprocants of extent 4 are as follows: deg. wt.

1. 0 a,

$2.24 M=4 a c-5 b^{2}$,

3. $3 A=a^{2} d-3 a b c+2 b^{3}$,

$3.4 \quad P_{4}=50 a^{2} e-175 a b d+28 a c^{2}+105 b^{2} c^{*}$,

4. $6\left(a^{2} c e\right)=800 a^{2} c e-1000 a b^{2} e-875 a^{2} d^{2}+2450 a b c d-1344 a c^{3}-35 b^{2} c^{2}$,

$5.8\left(a^{3} e^{2}\right)=625 a^{3} e^{2}-4375 a^{2} b d e-49700 a^{2} c^{2} e+128625 a b^{2} c e-78750 b^{4} e$

$+55125 a^{2} c d^{2}-61250 a b^{2} d^{2}-156800 a b c^{2} d+183750 b^{3} c d$

$+84868 a c^{4}-102165 b^{2} c^{3}$.

The similar list of invariants for the quartic is

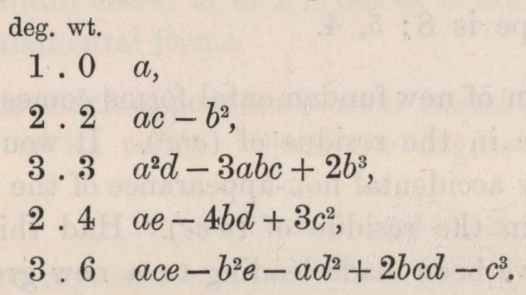

To obtain the fundamental forms of extent 4 we have to combine $M, A$ and the Quasi-Discriminant

$$
\left(a^{3} d^{2}\right)=125 a^{3} d^{2}-750 a^{2} b c d+500 a b^{3} d+256 a^{2} c^{3}+165 a b^{2} c^{2}-300 b^{4} c
$$

with the additional Protomorph

$$
P_{4}=50 a^{2} e-175 a b d+28 a c^{2}+105 b^{2} c
$$

* $P_{4}$ is the protomorph of minimum degree; the other protomorph, $B$, which will be used when we treat of Principiants, is, when expressed in terms of the irreducible forms,

$$
B=\frac{1}{50}\left(a P_{4}-128 M^{2}\right) .
$$


in such a manner that the combination contains a factor $a$. The removal of this factor gives rise to a form of lower degree, and the process is repeated as often as possible.

Calling that portion of any form which does not contain $a$ its residue, the residue of $4 M$ is $-5 b^{2}$, that of $\left(a^{3} d^{2}\right)$ being $-300 b^{4} c$, and that of $P_{4}$ being $105 b^{2} c$. Thus

$$
16 M P_{4}-7\left(a^{3} d^{2}\right)
$$

contains the factor $a$, and leads to $\left(a^{2} c e\right)$ of the type $6 ; 4,4$, which is the analogue to the Catalecticant

$$
\left|\begin{array}{lll}
a & b & c \\
b & c & d \\
c & d & e
\end{array}\right|
$$

The form $\left(a^{3} d^{2}\right)$ now ceases to be a groundform (=irreducible form) and is replaced by the Quasi-Catalecticant $\left(a^{2} c e\right)$, for

$$
\left(a^{3} d^{2}\right)=\frac{16}{7} M P_{4}-\frac{1}{7} a\left(a^{2} c e\right)
$$

Similarly, the Cubic Discriminant, a groundform $q u \hat{a}$ the letters $a, b, c, d$, becomes reducible when a new letter, $e$, is introduced, and is then replaced by the Catalecticant.

We now come to an extra form which has no analogue in invariants. The residue of the Quasi-Catalecticant $\left(a^{2} c e\right)$ is $-35 b^{2} c^{2}$, and consequently

$$
P_{4}^{2}-252 M\left(a^{2} c e\right)
$$

divides by a numerical multiple of $a$ (as it happens by $4 a$ ) and yields the form $\left(a^{3} e^{2}\right)$, whose type is $8 ; 5,4$.

Here the deduction of new fundamental forms comes to an end on account of the appearance of $e$ in the residue of $\left(a^{3} e^{2}\right)$. It would have ended sooner but for the apparently accidental non-appearance of the term $b^{3} d$ (of the same type $6 ; 4,4$ as $\left.b^{2} c^{2}\right)$ in the residue of $\left(a^{2} c e\right)$. Had this term appeared, no combination could have been made leading to a new groundform after $\left(a^{2} c e\right)$. We are able to show from à priori considerations that it cannot exist.

For the arguments in the annihilator $V$, up to $\partial_{e}$ inclusive, are

$$
a^{2} \partial_{b}, a b \partial_{c}, a c \partial_{d}, b^{2} \partial_{d}, a d \partial_{e} \text {, and } b c \partial_{e} \text {. }
$$

If, now, the term $\mu b^{3} d$ were to form part of a Pure Reciprocant, $b^{2} \partial_{d}$ operating upon it would give $\mu b^{5}$; but every other portion of the operator would necessarily give terms containing one or other of the letters $a, c$. Since such terms cannot destroy $\mu b^{5}$, we must have $\mu b^{5}=0$. Hence the term in question is necessarily non-existent. 
The method of combining the protomorphs which we have followed shows that the fundamental reciprocants of extent 4 are connected inter se by the two relations or syzygies

$$
\begin{gathered}
7\left(256 M^{3}+125 A^{2}\right)-16 a M P_{4}+a^{2}\left(a^{2} c e\right)=0 \\
P_{4}^{2}-252 M\left(a^{2} c e\right)-4 a\left(a^{3} e^{2}\right)=0 .
\end{gathered}
$$

The invariants of the binary quartic are connected by only one syzygy, similar to the first of these; the second has no analogue in the theory of Invariants. It has been shown that the irreducible reciprocants of extent 3 are connected by the syzygy

$$
256 M^{3}+125 A^{2}-a\left(a^{3} d^{2}\right)=0 .
$$

Substituting in this for the Quasi-Discriminant $\left(a^{3} d^{2}\right)$ its value expressed in terms of the fundamental forms of extent 4 , by means of the equation

$$
16 M P_{4}-7\left(a^{3} d^{2}\right)=a\left(a^{2} c e\right)
$$

we obtain the first of the above syzygies. By a precisely similar substitution, the syzygy connecting the invariants of the quartic is derived from the one which connects the invariants of the cubic.

Every reciprocant of extent 4 is a rational integral function of the six fundamental forms given in the table; and, by means of the syzygies, powers, but not products, of $A$ and $P_{4}$ can be removed from this function. For the first syzygy gives $A^{2}$ and the second gives $P_{4}{ }^{2}$ as a rational integral function of the four remaining forms $a, M,\left(a^{2} c e\right)$, and $\left(a^{3} e^{2}\right)$. Hence every reciprocant of extent 4 is of one or other of the forms

$$
\Phi, A \Phi, P_{4} \Phi, A P_{4} \Phi
$$

where $\Phi$ does not contain either $A$ or $P_{4}$, but is a rational integral function of the other four fundamental forms.

Let the four forms which appear in $\Phi$ occur raised to the powers $\kappa, \lambda, \mu, \nu$, respectively, in one of its terms. Since the degree-weights of these four forms are

$$
1.0,2.2,4.6 \text { and } 5.8 \text {, }
$$

any such term may be represented by

$$
a^{\kappa}\left(a^{2} x^{2}\right)^{\lambda}\left(a^{4} x^{6}\right)^{\mu}\left(a^{5} x^{8}\right)^{\nu}
$$

Thus the totality of the terms in $\Phi$ will be represented by

$$
\sum a^{\kappa}\left(a^{2} x^{2}\right)^{\lambda}\left(a^{4} x^{6}\right)^{\mu}\left(a^{5} x^{8}\right)^{\nu}=\frac{1}{(1-a)\left(1-a^{2} x^{2}\right)\left(1-a^{4} x^{6}\right)\left(1-a^{5} x^{8}\right)} .
$$

Now, $A, P_{4}$ and $A P_{4}$ have the degree-weights

$$
3.3,3.4 \text { and } 6.7 \text {, }
$$

and consequently the totality of terms in

$$
\Phi, A \Phi, P_{4} \Phi \text { and } A P_{4} \Phi
$$

S. IV. 
(that is, the totality of the pure reciprocants of extent 4 ) will be represented by

$$
\begin{aligned}
\left(1+a^{3} x^{3}+a^{3} x^{4}+a^{6} x^{7}\right) \sum a^{\kappa}\left(a^{2} x^{2}\right)^{\lambda}\left(a^{4} x^{6}\right)^{\mu}\left(a^{5} x^{8}\right)^{\nu} & \\
& =\frac{1+a^{3} x^{3}+a^{3} x^{4}+a^{6} x^{7}}{(1-a)\left(1-a^{2} x^{2}\right)\left(1-a^{4} x^{6}\right)\left(1-a^{5} x^{8}\right)} .
\end{aligned}
$$

Hence the number of Pure Reciprocants of the type $w ; i, 4$ is the coefficient of $a^{i} x^{w}$ in the expansion of a fraction whose numerator is

with the denominator

$$
1+a^{3} x^{3}+a^{3} x^{4}+a^{6} x^{7}
$$

$$
(1-a)\left(1-a^{2} x^{2}\right)\left(1-a^{4} x^{6}\right)\left(1-a^{5} x^{8}\right)
$$

This fraction is called the Representative Form of the Generating Function, in contradistinction to the Crude Form, which is a fraction with the numerator

having for its denominator

$$
1-a^{-1} x
$$

$$
(1-a)(1-a x)\left(1-a x^{2}\right)\left(1-a x^{3}\right)\left(1-a x^{4}\right) .
$$

The crude form expresses the fact that the number of pure reciprocants of the type

is

$$
w ; i, j
$$

$$
(w ; i, j)-(w-1 ; i+1, j)
$$

Its numerator is $1-a^{-1} x$ for all extents; for the general case in which the extent is $j$, its denominator consists of the $j+1$ factors

$$
(1-a)(1-a x)\left(1-a x^{2}\right) \ldots\left(1-a x^{j}\right) .
$$

The removal of the negative terms [corresponding to cases in which $(w ; i, j)<(w-1 ; i+1, j)]$ from the crude form would give either the representative form or one equivalent to it, according as the representative form is or is not in its lowest terms. In the parallel theory of Invariants the terms to be rejected are those for which $i j-2 w<0$; but we do not at present know of any similar criterion for reciprocants, and are thus unable to pass directly from the crude to the representative form of their generating function.

Knowing both the crude and the representative form for reciprocants of extent 4, we may verify that the difference between these two forms of the generating function is omninegative. It will be found that

$$
\begin{aligned}
\frac{1-a^{-1} x}{(1-a)(1-a x)\left(1-a x^{2}\right)\left(1-a x^{3}\right)\left(1-a x^{4}\right)} \\
=\frac{1+a^{3} x^{3}+a^{3} x^{4}+a^{6} x^{7}}{(1-a)\left(1-a^{2} x^{2}\right)\left(1-a^{4} x^{6}\right)\left(1-a^{5} x^{8}\right)} \\
-\frac{1}{\left(1-a x^{2}\right)\left(1-a x^{3}\right)\left(1-a x^{4}\right)}\left(\frac{a^{-1} x+a^{2} x^{5}}{1-a^{4} x^{6}}+\frac{x^{2}+a^{2} x^{6}}{1-a^{5} x^{8}}\right) \\
-\frac{1}{\left(1-a x^{4}\right)\left(1-a^{4} x^{6}\right)\left(1-a^{5} x^{8}\right)}\left(\frac{x+a^{5} x^{10}}{1-a x^{2}}+\frac{a^{3} x^{5}+a^{2} x^{7}}{1-a x^{3}}\right) .
\end{aligned}
$$


Thus the crude form is seen to consist of an omnipositive part, equal to the representative form, and an omninegative part.

There is no difficulty in obtaining the representative form of the generating function for pure reciprocants of extents 2 and 3 . In the one case every reciprocant is a rational integral function of two forms of degree-weight, 1.0 and 2.2 respectively. The generating function is therefore

$$
\frac{1}{(1-a)\left(1-a^{2} x^{2}\right)}
$$

In the other case (that is, for extent 3 ) every pure reciprocant can be expressed as a rational integral function of four forms, of which the degree-weights are $1.0,2.2,3.3$ and 5.6, no higher power than the first of the form 3.3 occurring in the function. Thus the representative form is

$$
\frac{1+a^{3} x^{3}}{(1-a)\left(1-a^{2} x^{2}\right)\left(1-a^{5} x^{6}\right)} \text {. }
$$

\section{LECTURE XVIII.}

The number of Pure Reciprocants of a given degree is finite; the number of Invariants of the same degree is infinite. Thus, for example, we have the well-known series of invariants

$$
a c-b^{2}, a e-4 b d+3 c^{2}, \ldots
$$

all of degree 2, but of weights and extents proceeding to infinity. This may be proved from the theory; of partitions (see American Journal of Mathematics, Vol. v., No. 1, "On Subinvariants," Excursus on Rational Fractions and Partitions). It will be seen in that article that if $N(w: i)$ is the number of ways in which $w$ can be divided into $i$ parts, and if $P$ is the least common multiple of $2,3,4, \ldots, i$, then $N(w: i)$ can be expressed under the form

$$
F(w, i)+F^{\prime}(w, i, p)
$$

where $p$ is the residue of $w$ in respect of $P$.

$$
\text { Writing } \quad w+\frac{i(i+1)}{4}=\nu,
$$

$F(w, i)$ is of the form

$$
\frac{\nu^{i-1}}{2^{2} \cdot 3^{2} \ldots(i-1)^{2} \cdot i}+\ldots
$$

all the succeeding indices of the powers of $\nu$ in $F(w, i)$ decreasing by 2 , and their coefficients being transcendental functions of $i$ which involve Bernoulli's Numbers.

In $F^{\prime}(w, i, p)$ the highest index of $\nu$ is one unit less than the number of times that $i$ is divisible by 2 , that is, is $\frac{i-2}{2}$ or $\frac{i-3}{2}$, according as $i$ is even or odd. 
Thus, for the partitions of $w$ into 3 parts, we have the formula

where

$$
N(w: 3)=\left\{\frac{\nu^{2}}{12}-\frac{7}{72}\right\}+\left\{\frac{1}{8}(-1)^{\nu+1}+\frac{1}{9}\left(\rho_{1}^{\nu}+\rho_{2}^{\nu}\right)\right\},
$$

And, for the partitions of $w$ into 4 parts,

$$
\begin{array}{r}
N(w: 4)=\left\{\frac{\nu^{3}}{144}-\frac{5 \nu}{96}\right\}+\left\{\frac{1}{32}(-)^{\nu+1} \nu+\frac{1}{27}\left(\rho_{1}^{\nu+1}+\rho_{2}{ }^{\nu+1}-\rho_{1}{ }^{\nu-1}-\rho_{2}^{\nu-1}\right)\right. \\
\left.-\frac{1}{32}\left(i_{1}^{\nu+1}+i_{2}^{\nu+1}-i_{1}^{\nu-1}-i_{2}^{\nu-1}\right)\right\},
\end{array}
$$

where

$$
\nu=w+\frac{1+2+3+4}{2}=w+5
$$

and

$$
\rho_{1}, \rho_{2} \text { are the roots of } \rho^{2}+\rho+1=0 \text {, }
$$

$$
i_{1}, i_{2} \quad \text { " " } i^{2}+1=0
$$

in other words, $\rho_{1}$ and $\rho_{2}$ are primitive cube roots, and $i_{1}, i_{2}$ primitive fourth roots of unity.

The principal term of $N(w: 3)$, regarded as a function of $w$, is

$$
\frac{w^{2}}{12}=\frac{w^{2}}{2^{2} \cdot 3}, \text { that of } N(w: 4) \text { being } \frac{w^{3}}{144}=\frac{w^{3}}{2^{2} \cdot 3^{2} \cdot 4} .
$$

And in general the principal term of $N(w: i)$ is

$$
\frac{w^{i-1}}{2^{2} \cdot 3^{2} \cdot 4^{2} \ldots(i-1)^{2} \cdot i}
$$

Hence it follows, from a general algebraical principle, that for all values of $w$ above a certain limit, which depends on the value of $i$ and may be determined by the aid of partition tables, $(w ; i, \infty)-(w-1 ; i+1, \infty)$ must become negative.

Ultimately, $\frac{(w-1 ; i+1, \infty)}{(w ; i, \infty)}=\frac{w}{i(i+1)}$, which must eventually be greater than unity. This shows that beyond a certain value of $w$ there can be no pure reciprocant, and consequently that the number of pure reciprocants of a given degree $i$ is finite.

Mr Hammond remarks that the formulae for $N(w: 3)$ and $N(w: 4)$ may, by the substitution of trigonometrical expressions for the roots of unity, accompanied by some easy reductions, be transformed into

$$
N(w: 3)=\frac{\nu^{2}}{12}+\frac{1}{4} \sin ^{2} \frac{\nu \pi}{2}-\frac{4}{9} \sin ^{2} \frac{\nu \pi}{3},
$$

and $\quad N(w: 4)=\frac{\nu^{3}}{144}-\frac{\nu}{12}+\frac{\nu}{16} \sin ^{2} \frac{\nu \pi}{2}+\frac{1}{8} \sin \frac{\nu \pi}{2}-\frac{2}{9 \sqrt{ } 3} \sin \frac{\nu \pi}{3}$,

where, in the first formula, $\nu=w+3$, and in the second $\nu=w+5$. He also obtains the principal term of $N(w: i)$ from first principles as follows : 
The partitions of $w$ into $i$ parts may be separated into two sets, the first containing at least one zero part in each of its partitions, the second consisting of partitions in which no zero part occurs.

Suppressing one zero part in each partition of the first set, we see that the number of partitions in which 0 occurs is $N(w: i-1)$. Diminishing each part by unity in those partitions which contain no zeros, their number is seen to be $N(w-i: i)$. The sum of these two numbers is $N(w: i)$, which is the total number of partitions, and consequently

$$
N(w: i)=N(w: i-1)+N(w-i: i) .
$$

Let the principal term of $N(w: i-1)$ be $\alpha w^{i-2}$, where $\alpha$ is independent of $w$, and write

Then

$$
\begin{gathered}
w=i x, N(w: i)=u_{x}, N(w-i: i)=u_{x-1} . \\
u_{x}-u_{x-1}=\alpha w^{i-2}+\ldots=\alpha i^{i-2} x^{i-2}+\ldots
\end{gathered}
$$

Hence, by a simple summation, we find

$$
u_{x}=\alpha i^{i-2}\left\{x^{i-2}+(x-1)^{i-2}+(x-2)^{i-2}+\ldots\right\}+\ldots .
$$

But, since only the principal term of $u_{x}$ is required, this summation may be replaced by an integration. Thus the principal term of $u_{x}$ is

$$
\alpha i^{i-2} \int x^{i-2} d x=\frac{\alpha i^{i-2} x^{i-1}}{i-1} .
$$

Restoring

$$
w=i x \text { and } N(w: i)=u_{x}
$$

we see that the principal term of $N(w: i)$ is $\frac{a w^{i-1}}{(i-1) i}$. Thus the principal term of $N(w: i)$ is found from that of $N(w: i-1)$ by multiplying it by

\begin{tabular}{|c|c|c|c|c|c|c|c|c|c|c|c|c|c|c|}
\hline & 1 & 2 & 3 & 4 & 5 & 6 & 7 & 8 & 9 & 10 & 11 & 12 & 13 & 14 \\
\hline 2 & 1 & 2 & 2 & 3 & 3 & 4 & 4 & 5 & 5 & 6 & 6 & 7 & 7 & 8 \\
\hline 3 & 1 & 2 & 3 & 4 & 5 & 7 & 8 & 10 & 12 & 14 & 16 & 19 & 21 & 24 \\
\hline 4 & 1 & 2 & 3 & 5 & 6 & 9 & 11 & 15 & 18 & 23 & 27 & 34 & 39 & 47 \\
\hline & 1 & 2 & 3 & 5 & 7 & 10 & 13 & 18 & 23 & 30 & 37 & 47 & 57 & 70 \\
\hline 6 & 1 & 2 & 3 & 5 & 7 & 11 & 14 & 20 & 26 & 35 & 44 & 58 & 71 & 90 \\
\hline
\end{tabular}

$$
\frac{w}{(i-1) i} \text {. }
$$

When $i=3$, the principal term is $\frac{w^{2}}{2^{2} \cdot 3}$; it is therefore $\frac{w^{3}}{2^{2} \cdot 3^{2} \cdot 4}$ when $i=4$; and for the general case it is $\frac{w^{i-1}}{2^{2} \cdot 3^{2} \cdot 4^{2} \ldots(i-1)^{2} \cdot i}$. table :

The value of $N(w: i)$ is given in line $i$ and column $w$ of the following 
From an inspection of the tabulated values of $N(w: i)$ we see that

$$
\begin{aligned}
& N(w: 2)-N(w-1: 3) \text { is negative or zero when } w>2 \text {, } \\
& N(w: 3)-N(w-1: 4) \quad, \quad, \quad w>6 \text {, } \\
& N(w: 4)-N(w-1: 5) \quad, \quad, \quad w>8, \\
& N(w: 5)-N(w-1: 6) \quad \# \quad \quad, \quad w>12 .
\end{aligned}
$$

Hence for pure reciprocants of indefinite extent, whose degrees are

$$
2,3,4,5 \text {, }
$$

the highest possible weights are $2,6,8$ and 12 , respectively.

In like manner, from Euler's table, in his memoir "De Partitione Numerorum " (published in 1750), it will be found that

\begin{tabular}{l|l|l|l|l|c|c|c|c|c|c|c|c|} 
for degrees & 2 & 3 & 4 & 5 & 6 & 7 & 8 & 9 & 10 & 11 & 12 & 13 \\
the highest weights are & 2 & 6 & 8 & 12 & 16 & 21 & 26 & 30 & 36 & 42 & 49 & 55
\end{tabular} .

Further than this the table, which goes up to $w=59$, will not enable us to proceed.

\begin{tabular}{|c|c|c|c|c|c|c|c|c|c|c|c|c|c|}
\hline & & & & & & EIG & T $w$ & & & & & & \\
\hline & 2 & 3 & 4 & 5 & 6 & 7 & 8 & 9 & 10 & 11 & 12 & 13 & 14 \\
\hline 2 & 1 & & & & & & & & & & & & \\
\hline 3 & 1 & 1 & 1 & & 1 & & & & & & & & \\
\hline 4 & 1 & 1 & 2 & 1 & 2 & 1 & 2 & & & & & & \\
\hline 5 & 1 & 1 & 2 & 2 & 3 & 2 & 4 & 3 & 4 & 2 & 3 & & \\
\hline
\end{tabular}

The actual number of pure reciprocants of degree $i$, weight $w$, and of indefinite extent, is seen in the following table, which gives the value of $N(w: i)-N(w-1: i+1)$ when positive, blank spaces being left in the table when this difference is zero or negative.

Thus, for degree 2 , there is only one pure reciprocant, namely

$$
(a c)=4 a c-5 b^{2} \text {. }
$$

For degree 3 the table shows that, in addition to the compound form

$$
a(a c)=a\left(4 a c-5 b^{2}\right),
$$

thereare three others whose weights are 3,4 and 6 respectively.

These are the three protomorphs,

$$
\begin{aligned}
& \left(a^{2} d\right)=a^{2} d-3 a b c+2 b^{3}, \\
& \left(a^{2} e\right)=50 a^{2} e-175 a b d+28 a c^{2}+105 b^{2} c, \\
& \left(a^{2} g\right)=14 a^{2} g-63 a b f-1350 a c e+1782 b^{2} e+1470 a d^{2}-4158 b c d+2310 c^{3} .
\end{aligned}
$$


With the above forms and $a$ we are able to form the following compounds of degree 4 :

$$
a^{2}(a c), a\left(a^{2} d\right),(a c)^{2}, a\left(a^{2} e\right), a\left(a^{2} g\right),
$$

whose weights are $2, \quad 3, \quad 4, \quad 4, \quad 6$.

The forms of degree 4 and weights $5,7,8$, and one of the forms of weight 6 , cannot be similarly made up of forms of inferior degree, and are therefore groundforms. Three of them are the protomorphs $\left(a^{3} f\right),\left(a^{3} h\right)$ and $\left(a^{3} i\right)$ of weights 5, 7 and 8, whose values were given in Lecture XVI. The groundform of weight 6 is the Quasi-Catalecticant given in the last lecture. All the forms of degree 4 have thus been accounted for except one of the two forms of weight 8 , which will be seen to be of extent 6 , and to have $a^{2} c g$ for its leading term.

We know from Euler's table that $N(8: 4)-N(7: 5)=2$; that is,

$$
(8 ; 4,8)-(7 ; 5,8)=2
$$

Now, $(8 ; 4,7)=N(8: 4)-1$, the omitted partition being 8.0 .0 .0 ,

$(8 ; 4,6)=N(8: 4)-2$, the partition $7 \cdot 1 \cdot 0.0$ being also left out,

$(8 ; 4,5)=N(8: 4)-4,\left\{\begin{array}{l}\text { for } 6.2 .0 .0 \text { and } 6.1 .1 .0 \text { are excluded from } \\ (8 ; 4,5), \text { but make their appearance in }(8 ; 4,6) .\end{array}\right.$

Similarly,

$$
(7 ; 5,7)=N(7: 5)
$$

$$
\begin{aligned}
& (7 ; 5,6)=N(7: 5)-1, \\
& (7 ; 5,5)=N(7: 5)-2 .
\end{aligned}
$$

We have, therefore,

$$
\begin{aligned}
& (8 ; 4,8)-(7 ; 5,8)=2 \\
& (8 ; 4,7)-(7 ; 5,7)=1 \\
& (8 ; 4,6)-(7 ; 5,6)=1 \\
& (8 ; 4,5)-(7 ; 5,5)=0
\end{aligned}
$$

Hence we may draw the following inferences:

(1) No pure reciprocant exists whose type is $8 ; 4,5$.

(2) The one whose type is 8;4,6 must contain the letter $g$.

(3) No fresh form is found by making the extent 7 instead of 6 , so that there is no pure reciprocant of weight 8 and degree 4 whose actual extent is 7 .

(4) There is a pure reciprocant (the Protomorph whose leading term is $a^{3} i$ ) whose actual extent is 8 .

(5) This, with the one whose actual extent is 6 , makes up the two given by $(8 ; 4,8)-(7 ; 5,8)=2$. 


\section{LECTURE XIX.}

The following is a complete list of the irreducible reciprocants of indefinite extent for the degrees 2,3 and 4 :

$$
\begin{aligned}
& \text { Deg. wt. } \\
& 2.2(a c), \\
& 3.3\left(a^{2} d\right), \\
& 3.4\left(a^{2} e\right), \\
& 3.6\left(a^{2} g\right), \\
& 4.5\left(a^{3} f\right), \\
& 4.6\left(a^{2} c e\right), \\
& 4.7\left(a^{3} h\right), \\
& 4.8\left(a^{3} i\right), \quad\left(a^{2} c g\right) .
\end{aligned}
$$

The values of all of them except $\left(a^{2} c g\right)$ have been given in previous lectures, and the method of obtaining them sufficiently indicated. Thus $(a c),\left(a^{2} d\right)$, $\left(a^{2} e\right),\left(a^{3} f\right),\left(a^{2} g\right),\left(a^{3} h\right)$ and $\left(a^{3} i\right)$ are the Protomorphs of minimum degree $P_{2}, P_{3}, P_{4}, P_{5}, P_{6}, P_{7}$ and $P_{8}$, respectively; and $\left(a^{2} c e\right)$ is the Quasi-Catalecticant whose value has been set forth in the table of irreducible forms of extent 4. It will be remembered that $\left(a^{2} c e\right)$ was found by combining the Quasi-Discriminant $\left(a^{3} d^{2}\right)$ with $P_{2} P_{4}$ linearly in such a manner that the combination, which is of the 5 th degree, divides by $a$ and gives $\left(a^{2} c e\right)$ of the 4 th degree. If we try to find $\left(a^{2} c g\right)$ by a similar process, it will be necessary to rise as high as the 7 th degree, and then to drop down by successive divisions by $a$ to the fourth.

In fact, since to a numerical factor près the residues of

are

$$
P_{2}, P_{3}, P_{4}, P_{5}
$$

that of

$b^{2}, \quad b^{3}, \quad b^{2} c, \quad b^{3} c$,

and that of

$$
\begin{aligned}
& P_{3} P_{5} \text { will be } b^{6} c \text {, } \\
& P_{2}^{2} P_{4} \text { will be } b^{6} c .
\end{aligned}
$$

Thus a linear combination of $P_{3} P_{5}$ and $P_{2}^{2} P_{4}$ will be divisible by $a$, and, taking account of the numerical coefficients, we shall find

$$
26 P_{2}^{2} P_{4}+875 P_{3} P_{5} \equiv 0(\bmod . a) \text {. }
$$

As a result of calculation, it will be seen that the above combination of the protomorphs divided by $a$,

$$
\frac{1}{a}\left(26 P_{2}^{2} P_{4}+875 P_{3} P_{5}\right)
$$

has (to a numerical factor près) the same residue as $P_{4}^{2}$. 
Making a second combination and division by $a$, we find

$$
7\left(\frac{26 P_{2}^{2} P_{4}+875 P_{3} P_{5}}{a}\right)-25 P_{4}^{2} \equiv 0(\bmod . a)=a S, \text { suppose. }
$$

Then, by actual calculation, the residue of $S$ is found to be

$$
-262500 b^{4} e+612500 b^{3} c d-339080 b^{2} c^{3} \text {. }
$$

Two reductions have already been made in obtaining this form $S$ of the 5 th degree. A final combination of $S$ with $P_{2} P_{6}$ and the form $\left(a^{3} e^{2}\right)$, whose value was given in a former lecture, enables us to divide out once more by $a$ and thus get the form $\left(a^{2} c g\right)$ of the 4 th degree.

It is the fact that $P_{2} P_{6}$ and $\left(a^{3} e^{2}\right)$ have residues which are not the same to a numerical factor près which necessitates the long calculation above described. No linear combination of $P_{2} P_{6}$ and $\left(a^{3} e^{2}\right)$ with one another is divisible by $a$, and it is necessary to find a third form $S$ a linear combination of which with both $P_{2} P_{6}$ and $\left(a^{3} e^{2}\right)$ will divide by $a$.

There is, however, another way of arriving at the form $\left(a^{2} c g\right)$ by using the eductive generator

$$
G=4\left(a c-b^{2}\right) \partial_{b}+5(a d-b c) \partial_{c}+6(a e-b d) \partial_{d}+\ldots
$$

Starting with the Quasi-Catalecticant

$$
\left(a^{2} c e\right)=800 a^{2} c e-1000 a b^{2} e-875 a^{2} d^{2}+2450 a b c d-1344 a c^{3}-35 b^{2} c^{2},
$$

and operating on it with $G$, we have

$$
\begin{aligned}
G\left(a^{2} c e\right) & =4\left(a c-b^{2}\right)\left(-2000 a b e+2450 a c d-70 b c^{2}\right) \\
& +5(a d-b c)\left(800 a^{2} e+2450 a b d-4032 a c^{2}-70 b^{2} c\right) \\
& +6(a e-b d)\left(-1750 a^{2} d+24.50 a b c\right) \\
& +7(a f-b e)\left(800 a^{2} c-1000 a b^{2}\right)
\end{aligned}
$$

The terms of this expression contain the common numerical factor 10 , which may be rejected; thus we have

$$
G\left(a^{2} c e\right)=10\left(a^{3} c f\right)
$$

where $\left(a^{3} c f\right)=560 a^{3} c f-700 a^{2} b^{2} f-650 a^{3} d e-290 a^{2} b c e+1500 a b^{3} e$

$$
+2275 a^{2} b d^{2}-1036 a^{2} c^{2} d-3710 a b^{2} c d+1988 a b c^{3}+63 b^{3} c^{2} \text {. }
$$

This form $\left(a^{3} c f\right)$ is the first educt of $\left(a^{2} c e\right)$, and is irreducible (but, being of the fifth degree, does not appear in our list, which contains no forms of higher degree than the fourth). Operating on it with $G$, we obtain the educt of $\left(a^{3} c f\right)$, which is the second educt of $\left(a^{2} c e\right)$. This second educt will be of the 6 th degree (its leading term will be $a^{4} \mathrm{cg}$ ), but is reducible to the 5 th when combined with

$$
\left(4 a c-5 b^{2}\right)\left(a^{2} c e\right)
$$

as we know from the general theorem concerning the reduction of second educts. We shall thus obtain a form $\left(a^{3} \mathrm{cg}\right)$, the reduced second educt of $\left(a^{2} c e\right)$, of the 5th degree, and a final combination of $\left(a^{3} \mathrm{cg}\right)$ with one or both of 
the forms $P_{2} P_{6}$ and $\left(a^{3} e^{2}\right)$ will enable us to divide once more by $a$ and thus arrive at $\left(a^{2} c g\right)$ of the 4 th degree.

By either of these methods we obtain

$$
\begin{aligned}
\left(a^{2} c g\right) & =1176 a^{2} c g-8085 a^{2} d f+7040 a^{2} e^{2}-1470 a b^{2} g+18963 a b c f \\
& -16940 a b d e-27160 a c^{2} e+26460 a c d^{2}-9555 b^{3} f \\
& +28098 b^{2} c e+12740 b^{2} d^{2}-52822 b c^{2} d+21560 c^{4}
\end{aligned}
$$

but the second way, besides being more direct, gives us at the same time the value of the irreducible form $\left(a^{3} c f\right)$.

Every Pure Reciprocant is an Invariant of a Binary Quantic whose coefficients $A, B, C, D, \ldots$ are functions of the original elements $a, b, c, d, \ldots$ such that

$$
\begin{aligned}
& V A=0 \\
& V B=A \\
& V C=2 B \\
& V D=3 C
\end{aligned}
$$

and conversely, every Invariant of this Binary Quantic, or of a system of such Binary Quantics, is a Pure Reciprocant.

This is a particular case of the more general theorem, due to $\mathrm{Mr}$ Hammond, that if $\Theta$ is the operator,

$$
\phi_{1}(a) \partial_{b}+\phi_{2}(a, b) \partial_{c}+\phi_{3}(a, b, c) \partial_{d}+\ldots
$$

where $\phi_{1}, \phi_{2}, \phi_{3}, \ldots$ are arbitrary rational integral functions, and if

$$
A, B, C, D, \ldots, A^{\prime}, B^{\prime}, C^{\prime}, D^{\prime}, \ldots, A^{\prime \prime}, B^{\prime \prime}, C^{\prime \prime}, \ldots
$$

be any rational integral functions of the original letters $a, b, c, \ldots$ which satisfy the conditions

$$
\begin{array}{lll}
\Theta A=0, & \Theta A^{\prime}=0, & \Theta A^{\prime \prime}=0, \\
\Theta B=A, & \Theta B^{\prime}=A^{\prime}, & \Theta B^{\prime \prime}=A^{\prime \prime}, \\
\Theta C=2 B, & \Theta C^{\prime}=2 B^{\prime}, & \Theta C^{\prime \prime}=2 B^{\prime \prime}, \\
\Theta D=3 C, & \Theta D^{\prime}=3 C^{\prime}, & \Theta D^{\prime \prime}=3 C^{\prime \prime},
\end{array}
$$

then every invariant in respect to the elements

$$
A, B, C, D, \ldots, A^{\prime}, B^{\prime}, C^{\prime}, D^{\prime}, \ldots, A^{\prime \prime}, B^{\prime \prime}, C^{\prime \prime}, D^{\prime \prime}, \ldots
$$

is a rational integral solution of the equation

$$
\Theta=0 \text {. }
$$

Obviously, every rational integral solution of $\Theta=0$ is an invariant in the above elements, so that the converse of the proposition is true. For the only 
conditions imposed upon $A, A^{\prime}, A^{\prime \prime}, \ldots$ are that they shall be rational integral functions of $a, b, c, d, \ldots$ annihilated by $\Theta$. Let

$$
\Phi\left(A, B, C, D, \ldots, A^{\prime}, B^{\prime}, C^{\prime}, D^{\prime}, \ldots, A^{\prime \prime}, B^{\prime \prime}, C^{\prime \prime}, D^{\prime \prime}, \ldots\right)
$$

be any invariant in the large letters. We have to show that

$$
\Theta \Phi=0 .
$$

$$
\text { Now, } \quad \begin{aligned}
\Theta \Phi & =\frac{d \Phi}{d A} \Theta A+\frac{d \Phi}{d B} \Theta B+\frac{d \Phi}{d C} \Theta C+\ldots \\
& +\frac{d \Phi}{d A^{\prime}} \Theta A^{\prime}+\frac{d \Phi}{d B^{\prime}} \Theta B^{\prime}+\frac{d \Phi}{d C^{\prime}} \Theta C^{\prime}+\ldots \\
& +\ldots \ldots \ldots, \ldots \ldots \ldots \ldots \ldots \ldots \ldots \ldots \ldots
\end{aligned}
$$

Hence, writing for $\Theta A, \Theta B, \Theta C, \ldots$, their values given above, we have

$$
\begin{aligned}
\Theta \Phi & =\left(A \partial_{B}+2 B \partial_{C}+3 C \partial_{D}+\ldots\right) \Phi \\
& +\left(A^{\prime} \partial_{B^{\prime}}+2 B^{\prime} \partial_{C^{\prime}}+3 C^{\prime} \partial_{D^{\prime}}+\ldots\right) \Phi \\
& +\ldots \ldots \ldots \ldots \ldots \ldots \ldots \ldots \ldots \ldots \ldots \ldots \ldots \ldots \ldots \ldots \ldots \ldots \ldots \ldots \ldots \ldots \ldots \ldots \ldots \\
& =0 \text { (since } \Phi \text { is an invariant }) ;
\end{aligned}
$$

which proves the proposition.

Confining our attention to a single set of letters, the Binary Quantic

$$
\left(A, B, C, \ldots J, K, L \gamma(x, y)^{n},\right.
$$

whose coefficients are formed from one another by the successive operation of $\Theta$ as above, may be called a Quasi-Covariant; and it will follow immediately from the Theory of Binary Forms that every Covariant of a Quasi-Covariant is itself a Quasi-Covariant, and that every Invariant of any Quasi-Covariant (or system of Quasi-Covariants) is an Invariant in respect to the letters $A, B, C, \ldots$, and therefore, by what precedes, a rational integral solution of $\Theta=0$.

Writing the terms of

$$
(A, B, C, \ldots J, K, L \gamma x, y)^{n}
$$

in reverse order, we have

$$
L y^{n}+n K x y^{n-1}+\frac{n(n-1)}{1.2} J x y^{n-2}+\ldots+A x^{n},
$$

where

$$
\Theta L=n K, \Theta K=(n-1) J, \ldots \Theta A=0 .
$$

Thus the Quasi-Covariant may be written

$$
L y^{n}+\Theta L x y^{n-1}+\frac{\Theta^{2} L}{1.2} x^{2} y^{n-2}+\ldots+\frac{\Theta^{n} L}{1.2 .3 \ldots n} x^{n}=y^{n}\left(e^{\frac{x \Theta}{y}}\right) L,
$$

where $\Theta^{n+1} L=0$.

This is the general symbolic expression for a Quasi-Covariant. An example of a Quasi-Covariant has already been given in Lecture II. [p. 310, above], 
where it was stated, and afterwards proved [p. 360], that the reciprocal of the $n$th modified derivative could be put under the form

$$
-t^{-n-3}\left(e^{\left.-\frac{V}{t}\right)} a_{n}\right.
$$

The numerator of this reciprocal expression, which may be called the reciprocal function, is

$$
t^{n}\left(e^{-\frac{V}{t}}\right) a_{n}
$$

which is identical with the general expression

if

$$
\begin{gathered}
y^{n}\left(\frac{x \Theta}{e^{\frac{x}{y}}}\right) L, \\
x=-1, y=t, L=a_{n} \text { and } \Theta=V .
\end{gathered}
$$

Hence every Invariant of the reciprocal function is a Pure Reciprocant.

This property of the reciprocal function was discovered independently by Mr C. Leudesdorf, who published his results in the Proceedings of the London Mathematical Society (Vol. xvII. p. 208). Mr Hammond's résults were given in two letters to me dated January 15th and January 20th, 1886, and were briefly alluded to by him at a meeting of the London Mathematical Society. They are here published for the first time.

Recalling the form of the operator

$$
\Theta=\phi_{1}(a) \partial_{b}+\phi_{2}(a, b) \partial_{c}+\phi_{3}(a, b, c) \partial_{d}+\ldots,
$$

where $\phi_{1}, \phi_{2}, \phi_{3}, \ldots$ are rational integral functions, we can form a QuasiCovariant of extent $j$ by a finite number of successive operations on a single letter of that extent.

To fix the ideas, take the letter $d$ of extent 3 , and operate on it with $\Theta$; then

$$
\Theta d=\phi_{3}(a, b, c) .
$$

Since $\phi_{1}, \phi_{2}, \phi_{3}, \ldots$ are by definition rational integral functions, we can, by operating a finite number of times with $\Theta$, remove first $c$ and then $b$ from $\phi_{3}(a, b, c)$, and thus obtain

$$
\Theta^{n} d=\text { funct. } a \text {, }
$$

where $n$ denotes a finite number of operations. Since $\Theta a=0$, we have

$$
\Theta^{n+1} d=0 .
$$

In this manner we form the Quasi-Covariant of the $n$th order

$$
y^{n}\left(e^{\frac{x \Theta}{y}}\right) d \text {. }
$$

If $\phi_{2}, \phi_{3}, \phi_{4}, \ldots$ do not contain higher powers than the first of the last letter in each, the order of the above Quasi-Covariant will be the same as its extent. This is the case with the reciprocal function, which is a co-reciprocant (that is, a Quasi-Covariant relative to $V$ ). 
Ex. $\quad y^{2}\left(e^{\frac{x V}{y}}\right) c=c y^{2}+V c x y+\frac{V^{2} c}{1.2} x^{2}=c y^{2}+5 a b x y+5 a^{3} x^{2}$.

The discriminant of this is the pure reciprocant

$$
5 a^{2}\left(a c-\frac{5 b^{2}}{4}\right) \text {. }
$$

As an additional example, consider the pair of linear co-reciprocants

$$
\begin{gathered}
4 a\left(4 a c-5 b^{2}\right) x+(5 a d-7 b c) y \\
50 a\left(a^{2} d-3 a b c+2 b^{3}\right) x+\left(25 a b d-32 a c^{2}+5 b^{2} c\right) y .
\end{gathered}
$$

The resultant of this pair is

$$
2 a\left(125 a^{3} d^{2}-750 a^{2} b c d+500 a b^{3} d+256 a^{2} c^{3}+165 a b^{2} c^{2}-300 b^{4} c\right),
$$

that is, is the Quasi-Discriminant multiplied by $2 a$.

\section{LECTURE XX}

"Quintessenced into a finer substance."-Drummond of Hawthornden.

Before proceeding with the proper subject of this day's lecture, I should like to mention a geometrical theorem which has fallen in my way, and which, inter alia, gives an immediate proof of the existence of 27 straight lines on a general cubic surface. It is proved by means of a Lemma (itself of quasi-geometrical origin) which finds its principal application in an extension of Bring's or Tschirnhausen's method, and shows how any number of specified terms, reckoning from either end, can be taken away from any equation of a sufficiently high degree*.

Subjectively speaking, I was led to the Lemma by considering the question, closely connected with Differential Invariants, of the method of depriving a linear differential equation of several terms.

Let $\phi$ be a cubic and $u$ a linear function in $x, y, z, t$, say

$$
\begin{aligned}
& \phi=a x^{3}+\ldots+f x^{2} y+\ldots \\
& u=l x+m y+n z+p t .
\end{aligned}
$$

Then, if $\psi$ is a scroll which contains all the straight lines on $\phi+\lambda u^{3}$, when the parameter $\lambda$ has any arbitrary numerical value from $+\infty$ to $-\infty$, I prove that

$$
\psi=\phi^{2} A+\phi u^{3} B+u^{6} C
$$

* I recover all Hamilton's results contained in his Report to the British Association, 1836, "On Jerrard's Method," in a much more clear and concise manner, and make important additions to his theory. 
where $\quad \psi$ is of the degree 15 in the variables $x, y, z, t$,

6 in the coefficients $(l, m, n, p)$ of $u$,

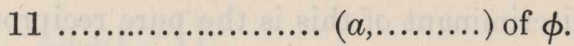

Or, more briefly, in

$\begin{array}{lll}x & l & a\end{array}$

$\psi$ is of degree

15611 , and consequently

C.................

9011.

The intersections of $\phi$ with $\psi$ are its intersections with $u^{6}$ and with $C$, of which the intersections with the arbitrary plane $u^{6}$ are clearly foreign to the question, but the cubic $\phi$ and the $9^{c} C$ intersect in 27 straight lines, which are the 27 ridges on $\phi$.

$C$ is identical with the covariant found by Clebsch and given in Salmon's Geometry of Three Dimensions at the end of the chapter on Cubic Surfaces. It may with propriety be called the Clebschian.

By giving the parameter $\lambda$ (which occurs in $\phi+\lambda u^{3}$ ) an infinitesimal variation, it is easily proved that

$$
B=-2 E C, A=E^{2} C, E^{3} C=0,
$$

where $E$ is the operator $l^{3} \partial_{a}+\ldots+3 l^{2} m \partial_{f}+\ldots$, which may be simply and completely defined by its property of changing the general cubic $\phi$ into $(l x+m y+n z+p t)^{3}$.

The equation $E^{3} C=0$ expresses a new property of the Clebschian: it shows that if $a, f$ are the coefficients of $x^{3}$ and any other term in $\phi$ containing $x^{2}$, neither $a^{3}$ nor $a^{2} f$ can occur in any one of the terms of $C$. Defining a principal term in $\phi$ as one which contains the cube of one of the variables, and a term adjacent to it as one which contains the square of the same variable, this is equivalent to saying that neither the cube of the coefficient of a principal term nor its square multiplied by the coefficient of any adjacent term can appear in any of the terms of $C$.

An interesting special case of the general theorem is when the arbitrary plane $u$ is taken to be one of the planes of reference, say $u=x$. Then

$$
l=1, \quad m=0, n=0, p=0,
$$

and the operator $E$ becomes simply $\frac{d}{d a}$. Thus we learn that

$$
\phi^{2} \frac{d^{2} C}{d a^{2}}-2 x^{3} \phi \frac{d C}{d a}+x^{6} C
$$

is a Scroll of the fifteenth order which contains all the Ridges on

$$
\phi+\lambda x^{3}
$$

for any arbitrary value of the parameter $\lambda$.

It also contains 6 times over the curve of intersection of $\phi=0$ with $x=0$. 
I now propose to give the substance, with a brief commentary, of some very interesting letters I have recently received from Capt. MacMahon. I abstain from giving a proof of his results, as I am informed that he intends to do this himself at an early meeting of the London Mathematical Society.

Using $V$ to signify the Reciprocant Annihilator and $\Omega$ the Annihilator of Invariants, we have studied the properties of

and those of

$$
\begin{aligned}
& V \frac{d}{d x}-\frac{d}{d x} V \\
& \Omega \frac{d}{d x}-\frac{d}{d x} \Omega
\end{aligned}
$$

These may be written in the form

$$
\left|\begin{array}{l}
V \frac{d}{d x} \\
V \frac{d}{d x}
\end{array}\right| \quad\left|\begin{array}{l}
\Omega \frac{d}{d x} \\
\Omega \frac{d}{d x}
\end{array}\right|
$$

and may be called alternants to $V, \frac{d}{d x}$ and to $\Omega, \frac{d}{d x}$ respectively.

It has been shown in Lecture VII. [p. 341, above] that

$$
V \frac{d}{d x}-\frac{d}{d x} V=2(3 i+w) a \text {. }
$$

The corresponding formula is

$$
\Omega \frac{d}{d x}-\frac{d}{d x} \Omega=3 i+2 w,
$$

as may be seen by writing $\kappa=0, \lambda=3, \mu=4, \nu=5, \ldots$ in a more general formula given in Lecture V. [p. 329, above].

Observe that operating with the alternant to $\Omega, \frac{d}{d x}$ is equivalent to multiplication by a number, and that operating with the alternant to $V, \frac{d}{d x}$ merely introduces a numerical multiple of $a$ as a factor. No such property exists for the Alternant

$$
V \Omega-\Omega V
$$

but one much more extraordinary.

MacMahon has found that this alternant, which he calls $J$, is a generator to a Reciprocant and a generator to an Invariant; that is, it converts a Reciprocant into another Reciprocant, and an Invariant into another Invariant. As regards a Differential Invariant, which is at once an Invariant and a Reciprocant, it is an Annihilator. He shows, in fact, that

and

$$
\begin{aligned}
& \Omega J-J \Omega=0 \\
& V J-J V=0 .
\end{aligned}
$$


If, then, $\Omega R=0$, it follows immediately that $\Omega(J R)=0$; that is, if $R$ is an invariant, $J R$ is so too. And in like manner, if

$$
V R=0, \quad V(J R)=0,
$$

that is, if $R$ is a reciprocant, so is $J R$.

Of course, if $M$ is a Differential Invariant,

$$
J M=V(\Omega M)-\Omega(V M)=0 .
$$

Let me here give a caution which may be necessary : The fact that a form is annihilated by $J$ is not sufficient to show that it is a Differential Invariant, though all Differential Invariants are necessarily annihilated by $J$. Forms exist which are subject to annihilation by

$$
J=a^{2} \partial_{c}+3 a b \partial_{d}+\ldots,
$$

but are, notwithstanding, neither invariants nor reciprocants.

Such a form is the monomial $b$, which is obviously annihilated by $J$. Another is $a d-3 b c$. For, since

$$
a^{2} d-3 a b c+2 b^{3}
$$

is a Differential Invariant, we have

But

$$
J\left(a^{2} d-3 a b c+2 b^{3}\right)=0 .
$$

therefore, also,

$$
J b^{3}=0 \text { and } J a=0 \text {; }
$$

$$
a J(a d-3 b c)=0 \text {. }
$$

The general theorem is as follows, and is a most remarkable $e_{m e}$ : If we write

$$
\begin{aligned}
m P(m, \mu, v, n)=\mu a^{m} \partial_{a_{n}} & +(\mu+v) m a^{m-1} b \partial_{a_{n+1}} \\
& +(\mu+2 v)\left(m a^{m-1} c+\frac{m(m-1)}{2} a^{m-2} b^{2}\right) \partial_{a_{n+2}} \\
& +(\mu+3 v)\left\{m a^{m-1} d+m(m-1) a^{m-2} b c\right. \\
& \left.+\frac{m(m-1)(m-2)}{6} a^{m-3} b^{3}\right\} \partial_{a_{n+3}}+\ldots
\end{aligned}
$$

where the coefficients of the terms inside the brackets are the same as those of the corresponding terms in the expansion of $(a+b+c+\ldots)^{m}$, and where $a_{n}$ stands for the $n$th letter of the series $a, b, c, d, \ldots$, then Capt. MacMahon establishes that the alternant of any two $P$ 's is another $P$.

A question here suggests itself naturally: What would be the alternant of three or more $P$ 's ? For instance, would the alternant

$$
\left|\begin{array}{lll}
P_{1} & P_{2} & P_{3} \\
P_{1} & P_{2} & P_{3} \\
P_{1} & P_{2} & P_{3}
\end{array}\right|=P_{1} P_{2} P_{3}-P_{1} P_{3} P_{2}+P_{2} P_{3} P_{1}-P_{2} P_{1} P_{3}+P_{3} P_{1} P_{2}-P_{3} P_{2} P_{1}
$$

be another $P$ ? *

* In my Multiple Algebra investigations, which I hope some day to resume, I have made important use of similar Alternants, which, it may be noticed, do not vanish when their elements 
Moreover, he obtains expressions for the parameters $m, \mu, v, n$ of the resulting $P$ in terms of the parameters of its two components. He proves that if $P_{1}, P_{2}$ are the two components whose alternant is $P$, supposing

$$
\begin{aligned}
& m_{1}, \mu_{1}, v_{1}, n_{1} \text { to be the parameters of } P_{1}, \\
& m_{2}, \mu_{2}, v_{2}, n_{2} \ldots \ldots \ldots \ldots \ldots \ldots \ldots \ldots \ldots P_{2},
\end{aligned}
$$

then the parameters $m, \mu, v, n$ of their resultant $P$ are given by the equations

$$
\begin{aligned}
& m=m_{1}+m_{2}-1, \\
& \mu=\left(m_{1}+m_{2}-1\right)\left\{\frac{\mu_{2}}{m_{2}}\left(\mu_{1}+n_{2} v_{1}\right)-\frac{\mu_{1}}{m_{1}}\left(\mu_{2}+n_{1} v_{2}\right)\right\}, \\
& v=\left(n_{2}-n_{1}\right) v_{1} v_{2}-\frac{m_{2}-1}{m_{1}} \mu_{1} v_{2}+\frac{m_{1}-1}{m_{2}} \mu_{2} v_{1}, \\
& n=n_{1}+n_{2} .
\end{aligned}
$$

It will be seen that $\Omega$ and $V$ are special forms of $P$. Thus,

$$
\begin{aligned}
& \Omega=P(1,1,1,1), \\
& V=P(2,4,1,1) .
\end{aligned}
$$

Now, if the second and third parameters are zero, every term of $P$ vanishes, and MacMahon finds that in the following two cases the second and third parameters of the resultant above given vanish.

(1) Supposing $\frac{\mu_{1}}{m_{1} v_{1}}$ to be an integer, this takes place when the two component systems of parameters are

(2) When they are

$$
\begin{aligned}
& m_{1}, \mu_{1}, \quad v_{1}, \quad n_{1}, \\
& m_{2}, \mu_{1} m_{2}, m_{1} v_{1}, n_{1}+\frac{\mu_{1}}{m_{1} v_{1}}\left(m_{2}-m_{1}\right) .
\end{aligned}
$$

$$
\begin{aligned}
& m_{1}, \mu_{1}, \quad v_{1}, \quad n_{1}, \\
& m_{2}, n_{1} m_{2}, m_{1}-1, \frac{\mu_{1}}{m_{1} v_{1}}\left(m_{2}-1\right) .
\end{aligned}
$$

Now,

$$
\begin{aligned}
& P(1,1,1,1)=\Omega, \\
& P(2,4,1,1)=V,
\end{aligned}
$$

and by the law of composition

$$
J=\Omega V-V \Omega=P(2,2,1,2) .
$$

Also,

$$
\left.\begin{array}{l}
2,2,1,2 \\
1,1,1,1
\end{array}\right\} \text { will be found to come under the first case; }
$$

and

$$
\left.\begin{array}{l}
2,2,1,2 \\
2,4,1,1
\end{array}\right\}
$$
the second.

are non-commutative. In this connection it is well worthy of observation that the $P$ 's (as indeed would be true of any operators linear in the differential inverses) obey the associative law.

It would be interesting to ascertain under what arithmetical conditions, if any, other than MacMahon's, any two linear operators of the same general form as his $P$ 's become commutative.

Perhaps it would also be worthy of inquiry whether the $P$ theory might not admit of extension in some form to operators non-linear in the differential inverses, and whether to every such operator of degrees $i$ and $j$ in the letters and their differential inverses there is not correlated another in which $i$ and $j$ are interchanged.

S IV. 
Hence,

$$
\Omega J-J \Omega=0 \text { and } V J-J V=0 .
$$

The above theorem is one of extraordinary beauty, and must play an important part in the future of Algebra.

In another letter Capt. MacMahon calls my attention to the fact that the operator called by me Cayley's generator $P$, in Lecture IV. of this course [p. 323, above], is a particular case of one of a much more general character given by him in the Quarterly Mathematical Journal (Vol. xx., p. 362).

He also states that every pure reciprocant, when multiplied by the needful power of $a$, is an invariant of the binary quantic

$$
\begin{aligned}
\{2 .(2 n+1) !\} a^{n+1}-n\{1 !(2 n+1) !\} & a^{n-1} b t \\
& +\frac{n(n-1)}{1.2}\{2 !(2 n) !\}\left\{a^{n-2} c+\frac{n-2}{2} a^{n-3} b^{2}\right\} t^{2}
\end{aligned}
$$

which I have written in the non-homogeneous form.

But this expression is (to a numerical factor près) identical with the numerator of $\frac{d^{n+2} x}{d y^{n+2}}$ when $t, a, b, \ldots$ are taken to be the modified differential derivatives $\frac{d y}{d x}, \frac{1}{2} \frac{d^{2} y}{d x^{2}}, \frac{1}{2.3} \frac{d^{3} y}{d x^{3}}, \ldots$. See my note on Burman's law for the Inversion of the Independent Variable [Vol. II. of this Reprint, p. 44].

The property that its invariants are pure reciprocants has already been proved in the lectures [above, p. 412].

\section{LECTURE XXI.}

I take blame to myself for not earlier communicating to the class the substance of a note of Mr Hammond's under date of January 20th, 1886, in which he makes an interesting application of the theorem that any invariant of the form

$$
y^{n}\left(e^{\frac{x}{y} V}\right) F(a, b, c, \ldots)
$$

in which the function $F$ is subject to the condition

$$
V^{n+1} F=0,
$$

or of any combination of such forms, is a pure reciprocant. 
Forms such as the above, whose invariants are pure reciprocants, he calls co-reciprocants. It follows that any covariant of one or more co-reciprocants is itself a co-reciprocant, for any invariant of a covariant is an invariant.

Taking $F$ to be a single letter $b, c, d$, he forms the functions

$$
\begin{aligned}
& b y+2 a^{2} x \\
& c y^{2}+5 a b x y+5 a^{3} x^{2} \\
& d y^{3}+3\left(2 a c+b^{2}\right) x y^{2}+21 a^{2} b x^{2} y+14 a^{4} x^{3}
\end{aligned}
$$

in which

$$
\begin{aligned}
2 a^{2} & =V b, \\
5 a b & =V c, 5 a^{3}=\frac{V^{2} c}{1.2}, \\
3\left(2 a c+b^{2}\right) & =V d, \quad 21 a^{2} b=\frac{V^{2} d}{1.2}, \quad 14 a^{4}=\frac{V^{3} d}{1.2 .3} .
\end{aligned}
$$

On writing $y=t, x=-1$, it will be observed that these three forms are the numerators of

$$
\frac{1}{3 !} ! \frac{d^{3} x}{d y^{3}}, \frac{1}{4 !} \frac{d^{4} x}{d y^{4}}, \frac{1}{5 !} \frac{d^{5} x}{d y^{5}} .
$$

The Jacobian of (1) and (2) is

$$
\left(4 a c-5 b^{2}\right) a y
$$

the coefficient of $a y$ is the familiar pure reciprocant $4 a c-5 b^{2}$.

The Jacobian of (1) and (3) is the determinant

$$
\left|\begin{array}{ll}
b & 2 a^{2} \\
d y^{2}+\left(4 a c-5 b^{2}\right) x y & \left(2 a c+b^{2}\right) y^{2}
\end{array}\right|
$$

which is divisible by $y$, giving the quotient

$$
\left(2 a^{2} d-2 a b c-b^{3}\right) y+2 a^{2}\left(4 a c-5 b^{2}\right) x .
$$

This is

$$
y\left(e^{\frac{x}{y} V}\right)\left(2 a^{2} d-2 a b c-b^{3}\right)
$$

the terms involving $\frac{x^{2}}{y}, \frac{x^{3}}{y^{2}}, \ldots$ vanishing identically.

Looking at $2 a^{2} d-2 a b c-b^{3}$ as the anti-source to a Co-reciprocant*, we might at first sight expect that it would give rise to a co-reciprocant of the third order in $x, y$, whereas we see it is the anti-source of a linear coreciprocant.

* What differentiates Reciprocants from Invariants is that we have no reverser to $V$ as $O$ is to $\Omega$ in the theory of Invariants, that is, no reverser which does not introduce an additional letter.

The coefficients of a covariant are obtained either from the source by continually operating with $O$, or from the anti-source by continually operating with $\Omega$. But in the case of a co-reciprocant, we are only able to proceed in one direction (namely from the anti-source, or coefficient of the highest power of $y$, to the source), as we have only one operator, $V$, at our disposal.

$$
27-2
$$


We have $\quad V\left(2 a^{2} d-2 a b c-b^{3}\right)=2 a^{2}\left(4 a c-5 b^{2}\right)$.

Combining this with

$$
V\left(a^{2} d-3 a b c+2 b^{3}\right)=0 \quad \text { (the well-known Mongian), }
$$

and dividing by $a$, he obtains

$$
\begin{array}{lr} 
& V(5 a d-7 b c)=4 a\left(4 a c-5 b^{2}\right) . \\
\text { Hence } & (5 a d-7 b c) y+4 a\left(4 a c-5 b^{2}\right) x
\end{array}
$$

is a co-reciprocant. It is in fact (4) reduced in degree.

The Jacobian of (5) and of $c y^{2}+5 a b x y+5 a^{3} x^{2}$, that is,

$$
\left|\begin{array}{ll}
5 a d-7 b c & 4 a\left(4 a c-5 b^{2}\right) \\
2 c y+5 a b x & 5 a b y+10 a^{3} x
\end{array}\right|
$$

will divide by $a$, and gives the new linear co-reciprocant

$$
\left(25 a b d-32 a c^{2}+5 b^{2} c\right) y+50 a\left(a^{2} d-3 a b c+2 b^{3}\right) x .
$$

The coefficient of $y$ is of weight 4 , but instead of giving rise to a coreciprocant of the 4 th order, we see that this again is the anti-source of a linear co-reciprocant.

The resultant of the two linear co-reciprocants (4) and (6) divided by a numerical multiple of $a$ gives the well-known Quasi-Discriminant $125 a^{3} d^{2}+\ldots$, as was stated at the end of Lecture XIX [above, p. 413].

The noticeable fact is that (including $b y+2 a^{2} x$ ) there exist 3 linear independent co-reciprocants of extent 3. Probably there are no more, but this requires proof.

The promised land of Differential Invariants or Projective Reciprocants is now in sight, and the remainder of the course will be devoted to its elucidation. Twenty lectures have been given on the underlying matter, and probably ten more, at least, will have to be expended on this higher portion of the theory.

One is surprised to reflect on the change which has come over the face of Algebra in the last quarter of a century. It is now possible to enlarge to an almost unlimited extent on any branch of it. These thirty lectures, embracing only a fragment of the theory of reciprocants, might be compared to an unfinished epic in thirty cantos. Does it not seem as if Algebra had attained to the character of a fine art, in which the workman has a free hand to develop his conceptions as in a musical theme or a subject for painting? Formerly it consisted almost exclusively of detached theorems, but now-adays it has reached a point in which every properly developed algebraical composition, like a skilful landscape, is expected to suggest the notion of an infinite distance lying beyond the limits of the canvas.

It is quite conceivable that the results we have been investigating may be descended upon from a higher and more general point of view. Many 
circumstances point to such a consummation being probable. But man must creep before he can walk or run, and a house cannot be built downwards from the roof. I think the mere fact that our work enables us to simplify and extend the results obtained by so splendid a genius as M. Halphen, is sufficient to convey to us the assurance that we have not been beating the wind or chasing a phantom, but doing solid work. Let me instance one single point: M. Halphen has succeeded, by a prodigious effort of ingenuity, in obtaining the differential equation to a cubic curve with a given absolute invariant. His method involves the integration of a complicated differential equation. In the method which I employ the same result is obtained by a simple act of substitution in an exceedingly simple special form of Aronhold's $S$ and $T$, capable of being executed in the course of a few minutes on half a sheet of paper, without performing any integration whatever. This will be seen to be a simple inference from the theorem invoked under three names, to which allusion has been made in a preceding lecture and the demonstration of which will shortly occupy our attention.

Before entering upon the theory of Differential Invariants, I think it desirable to bring forward the exceedingly valuable and interesting communication with which I have been favoured by M. Halphen establishing $\grave{a}$ priori the existence of invariants in general.

\section{Sur L'Existence Des Invariants.}

\section{(Extracted from a Letter of M. Halphen to Professor Sylvester.)}

Dans des théories diverses on a rencontré des Invariants sans qu'on ait pénétré la cause générale de leur existence. C'est cette lacune qu'il s'agit ici de faire disparaître.

1. Soient $A, B, \ldots, L$ des quantités auxquelles on puisse attribuer des valeurs ad libitum.

Une substitution consiste à remplacer ces quantités $(A, B, \ldots, L)$ par d'autres $(a, b, \ldots, l)$.

Les substitutions, que l'on doit considérer ici, sont définies par des relations algébriques, de forme supposée donnée, mais contenant des paramètres arbitraires $p, q, \ldots$

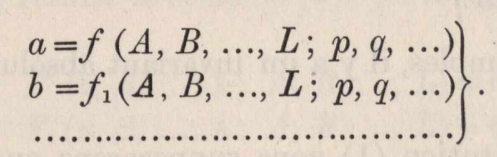

Soit maintenant une seconde substitution, de même espèce, mais avec d'autres paramètres $\pi, \chi, \ldots$, et donnant lieu à $(\alpha, \beta, \ldots, \lambda)$, en sorte qu'on ait

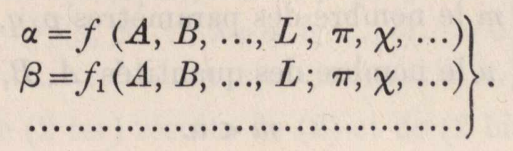


2. Définition. Les substitutions dont il s'agit forment un GROUPE, si, quels que soient les paramètres $p, q, \ldots, \pi, \chi, \ldots$, ainsi que $A, B, \ldots, L$, il existe des quantités $P, Q, \ldots$ vérifiant les égalités semblables

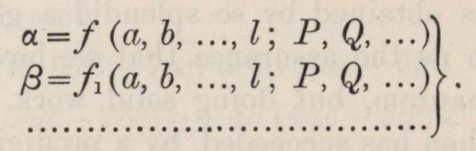

Les invariants sont l'apanage exclusif des substitutions formant groupe. On va le montrer. Mais auparavant, pour éviter toute confusion, on doit faire une remarque sur la définition.

3. Dans les diverses théories où l'on a rencontré des Invariants, les substitutions forment groupe, en effet, suivant cette définition; mais il s'y rencontre encore une circonstance particulière de plus, c'est que les paramètres $P, Q, \ldots$ de la substitution composée (1 ter) dépendent uniquement des paramètres $p, q, \ldots, \pi, \chi, \ldots$ des substitutions composantes $(1)$ et ( 1 bis). Cette propriété n'est pas nécessaire à l'existence des Invariants, et nous ne la supposerons pas ici. Il sera donc entendu que $P, Q, \ldots$ peuvent dépendre, non seulement de $p, q, \ldots, \pi, \chi, \ldots$, mais aussi de $A, B, \ldots, L$.

\section{Exemples :}

I.

$$
\begin{aligned}
& a=A p^{2}, \quad b=A p q+B p, \quad c=A q^{2}+2 B q+C ; \\
& \alpha=A \pi^{2}, \quad \beta=A \pi \chi+B \pi, \quad \gamma=A \chi^{2}+2 B \chi+C ; \\
& \alpha=a P^{2}, \quad \beta=a P Q+b P, \quad \gamma=a Q^{2}+2 b Q+c ; \\
& P=\frac{\pi}{p}, \quad Q=\frac{\chi-q}{p} .
\end{aligned}
$$

$P$ et $Q$ ne dépendent pas de $A, B, C$.

$$
\text { II. } \begin{array}{lll}
a=A^{3} p^{2}, & b=A^{2} p q+A B p, & c=A q^{2}+2 B q+C ; \\
\alpha=A^{3} \pi^{2}, & \beta=A^{2} \pi \chi+A B \pi, & \gamma=A \chi^{2}+2 B \chi+C ; \\
\alpha=a^{3} P^{2}, & \beta=a^{2} P Q+a b P, & \gamma=a Q^{2}+2 b Q+c ; \\
& P=\frac{\pi}{A^{3} p^{3}}, \quad Q=\frac{\chi-q}{A p} .
\end{array}
$$

$P$ et $Q$ dépendent de $A$.

Dans ces deux exemples, il y a un invariant absolu, $\frac{B^{2}-A C}{A}$.

4. Dans la substitution (1) nous supposerons que le nombre des paramètres soit inférieur au nombre des quantités $A, B, \ldots, L$.

Soient ainsi $\quad m$ le nombre des paramètres $p, q, \ldots$, $n$ le nombre des quantités $A, B, \ldots, L$, 
Cela étant, on peut éliminer les paramètres entre les équations (1), et il reste $(n-m)$ équations

$$
\left.\begin{array}{l}
F(a, b, \ldots, l ; A, B, \ldots, L)=0 \\
F_{1}(a, b, \ldots, l ; A, B, \ldots, L)=0 \\
\ldots \ldots \ldots \ldots \ldots \ldots \ldots \ldots \ldots \ldots \ldots . . .
\end{array}\right\}
$$

THÉORÈme: Si les substitutions considérées forment GROUPE, les $(n-m)$ équations (2) peuvent être mises sous la forme

$$
\begin{aligned}
& \Phi(a, b, \ldots, l)=\Phi(A, B, \ldots, L) \\
& \left.\Phi_{1}(a, b, \ldots, l)=\Phi_{1}(A, B, \ldots, L)\right\} \text {, }
\end{aligned}
$$

en d'autres termes, il y a $(n-m)$ invariants absolus.

Réciproquement, s'il y a $(n-m)$ invariants absolus (distincts), les substitutions forment groupe.

5. Démonstration. Prouvons d'abord la seconde partie, ou réciproque. Voici l'hypothèse : des équations (1), par élimination de $p, q, \ldots$ resultent les équations (3).

Par conséquent, $A, B, \ldots, L$ et $a, b, \ldots, l$ étant quelconques, mais satisfaisant aux équations (3), on peut déterminer $p, q$, au moyen des équations (1).

Soient $A, B, \ldots, L, p, q, \ldots, \pi, \chi, \ldots$ pris arbitrairement, et $a, b, \ldots, l$, $\alpha, \beta, \ldots, \lambda$ déterminés par $(1)$ et $(1 \mathrm{bis})$. Suivant l'hypothèse, on a

$$
\Phi(a, b, \ldots, l)=\Phi(A, B, \ldots, L) \text { et } \Phi(\alpha, \beta, \ldots, \lambda)=\Phi(A, B, \ldots, L) \text {; }
$$

donc

$$
\Phi(a, b, \ldots, l)=\Phi(\alpha, \beta, \ldots, \lambda) \text {, etc. }
$$

Donc on peut déterminer $P, Q, \ldots$ par les équations (1 ter), ce qu'il fallait démontrer.

Démontrons maintenant la première partie, ou théorème direct. Par hypothèse, $A, B, \ldots, L, p, q, \ldots, \pi, \chi, \ldots$ étant pris à volonté et $a, b, \ldots, l$, $\alpha, \beta, \ldots, \lambda$ déterminés au moyen de $(1)$ et $(1 \mathrm{bis})$, il en résulte les relations (1 ter).

Des équations (1) résulte le système (2); de même, de (1 bis) et de (1 ter) resultent

$$
\left.\begin{array}{l}
F(\alpha, \beta, \ldots, \lambda ; A, B, \ldots, L)=0 \\
F_{1}(\alpha, \beta, \ldots, \lambda ; A, B, \ldots, L)=0 \\
\ldots \ldots \ldots \ldots \ldots \ldots \ldots \ldots \ldots \ldots \ldots \ldots . . .
\end{array}\right\},
$$

Je dis que le système ( 2 ter) résulte de $(2)$ et de $(2$ bis $)$. 
En effet, $a, b, \ldots, l$ et $\alpha, \beta, \ldots, \lambda$ n'étant definis que par (1) et (1 bis), le système $(2$ ter) résulte de $(1)$ et de $(1$ bis $)$ par l'élimination de $p, q, \ldots$, $\pi, \chi, \ldots$ et $A, B, \ldots, L$. Mais l'élimination de $p, q, \ldots$ remplace le système (1) par le système (2), celle de $\pi, \chi, \ldots$ remplace le système (1 bis) par (2 bis); donc ( 2 ter) résulte de l'élimination de $A, B, \ldots, L$ entre ( 2 ) et ( 2 bis).

Le système (2), ( 2 bis) est formé par $2(n-m)$ équations, et cependant l'élimination de $n$ lettres $A, B, \ldots, L$, au lieu de donner $(n-2 m)$ équations, en donne $(n-m)$, les équations (2 ter). Si donc on élimine seulement $(n-m)$ lettres $A, B, \ldots, G$, les $m$ autures $H, \ldots, L$ disparaîtront d'elles-mêmes. Tirons $A, B, \ldots, G$ des équations (2), et nous aurons

$$
\begin{aligned}
& A=\Psi(a, b, \ldots, l ; H, \ldots, L), \\
& B=\Psi_{1}(a, b, \ldots, l ; H, \ldots, L),
\end{aligned}
$$

Tirons de même $A, B, \ldots, G$ des équations ( 2 bis), et nous aurons

$$
\begin{aligned}
& A=\Psi(\alpha, \beta, \ldots, \lambda ; H, \ldots, L), \\
& B=\Psi_{1}(\alpha, \beta, \ldots, \lambda ; H, \ldots, L),
\end{aligned}
$$

Le résultat de l'élimination est donc représenté par $(n-m)$ équations telles que

$$
\begin{aligned}
& \Psi(a, b, \ldots, l ; H, \ldots, L)=\Psi(\alpha, \beta, \ldots, \lambda ; H, \ldots, L)) \\
& \left.\Psi_{1}(a, b, \ldots, l ; H, \ldots, L)=\Psi_{1}(\alpha, \beta, \ldots, \lambda ; H, \ldots, L)\right\} \text {, }
\end{aligned}
$$

et l'on sait que $H, \ldots, L$ disparaissent, d'eux-mêmes, de ces équations.

En assignant donc à $H, \ldots, L$ des valeurs numériques à volonté, on voit donc bien que les équations résultantes, équivalentes à ( 2 ter), ont la forme

$$
\begin{aligned}
& \Phi(a, b, \ldots, l)=\Phi(\alpha, \beta, \ldots, \lambda), \\
& \Phi_{1}(a, b, \ldots, l)=\Phi_{1}(\alpha, \beta, \ldots, \lambda),
\end{aligned}
$$

C'est ce qu'il fallait démontrer.

6. Remarques. Si les équations (4) sont rationnelles, la disparition de $H, \ldots, L$ exige que $\Psi$ ait la forme suivante

$$
\Psi=\Phi(a, b, \ldots, l) \Theta(H, \ldots, L)+\theta(H, \ldots, L),
$$

et de même pour $\Psi_{1}$, etc. Sous cette forme, on voit que $\Theta$ et $\theta$ disparaissent dans les équations (4), et l'invariant résultant est $\Phi$.

Mais, si les équations (4) sont irrationnelles, la disparition de $H, \ldots, L$ peut n'être pas immédiate. En assignant à $H, \ldots, L$ des valeurs numériques à volonté, comme on l'a dit dạs la démonstration, c'est-à-dire en considérant $H, \ldots, L$ comme des constantes arbitraires, on voit les invariants se présenter 
avec des constantes arbitraires. Ceci ne doit pas étonner, puisqu'il s'agit ici d'invariants absolus, que l'on peut effectivement modifier en leur ajoutant des constantes arbitraires ou en les multipliant par des constantes arbitraires, sans troubler la propriété d'invariance.

L'analyse employée dans la démonstration fournit un moyen régulier de former les invariants; ce moyen consiste à éliminer les paramètres dans les équations (1), puis à résoudre par rapport à $(n-m)$ quantités $A, B, \ldots, G$. Mais, les substitutions formant groupe, on peut aussi résoudre par rapport à $a, b, \ldots, g$, en éliminant les paramètres.

Exemple: $\quad a=A p^{2}, \quad b=A p q+B p, c=A q^{2}+2 B q+C$.

En résolvant par rapport à $c$, c'est-à-dire en tirant $p, q$ des deux premières, on obtient

$$
c=A\left(\frac{b-B p}{A p}\right)^{2}+2 B \frac{b-B p}{A p}+C=\frac{b^{2}}{A p^{2}}+C-\frac{B^{2}}{A}=\frac{b^{2}}{a}+C-\frac{B^{2}}{A} .
$$

Voici l'invariant $C-\frac{B^{2}}{A}$.

En résolvant par rapport à $b$, on trouve $b=\sqrt{ } a \sqrt{ }\left(\frac{B^{2}-A C}{A}\right)+c$, ce qui donne l'invariant $\frac{B^{2}-A C}{A}+c$, où $c$ est une constante arbitraire.

\section{LECTURE XXII.}

E pur si muove.

The theory still moves on. We have now emerged from the narrows and are entering on the mid-ocean of Differential Invariants, or of Principiants, as I have called them. These, it will now be seen, are perfectly defined by their property of being at one and the same time invariants and pure reciprocants. In other words, if $P$ be a Principiant, it has both $\Omega$ and $V$ for its annihilators. Thus, for example, the Mongian

$$
A=a^{2} d-3 a b c+2 b^{3}
$$

is necessarily a Principiant. For

$$
\Omega A=\left(a \partial_{b}+2 b \partial_{c}+3 c \partial_{d}\right)\left(a^{2} d-3 a b c+2 b^{3}\right)=0,
$$

and at the same time

$$
V A=\left\{2 a^{2} \partial_{b}+5 a b \partial_{c}+\left(6 a c+3 b^{2}\right) \partial_{d}\right\}\left(a^{2} d-3 a b c+2 b^{3}\right)=0 .
$$

Among Pure Reciprocants, those only are entitled to rank as Principiants whose form is persistent (merely taking up an extraneous factor, but otherwise unchanged) under the most general homographic substitution (see 
Lecture XIII. [pp. 379, 382 above]. We have therefore to show that such reciprocants and no others are subject to annihilation by $\Omega$.

With this end in view, let us consider the effect of substituting $\frac{x}{1+h x}$ for $x$ and $\frac{y}{1+h x}$ for $y$ in any rational integral function of $y$ and its derivatives with respect to $x$. Suppose that, in consequence of this substitution, the function

becomes changed into

$$
F\left(y, y_{1}, y_{2}, y_{3}, \ldots y_{n}\right)
$$

$$
F_{1}\left(x, y, y_{1}, y_{2}, y_{3}, \ldots y_{n}\right)
$$

then the transformed function will be

$$
F\left(Y, Y_{1}, Y_{2}, Y_{3}, \ldots Y_{n}\right)
$$

where $X=\frac{x}{1+h x}, Y=\frac{y}{1+h x}$, and $Y_{1}, Y_{2}, Y_{3}, \ldots Y_{n}$ are the successive derivatives of $Y$ with respect to $X$.

If, for the moment, we agree to consider $h$ as an infinitesimal (we shall afterwards give it a finite value), neglecting squares and higher powers of $h$, we may write

$$
\begin{aligned}
& \dot{X}=x-h x^{2}, \\
& Y=y-h x y .
\end{aligned}
$$

Hence, by $n$ successive differentiations of $Y$ with respect to $X$, neglecting squares of $h$ whenever they occur, we deduce

$$
\begin{aligned}
& Y_{1}=y_{1}+h x y_{1}-h y, \\
& Y_{2}=y_{2}+3 h x y_{2} \text {, } \\
& Y_{3}=y_{3}+5 h x y_{3}+3 h y_{2} \text {, } \\
& Y_{4}=y_{4}+7 h x y_{4}+8 h y_{3} \text {, } \\
& Y_{5}=y_{5}+9 h x y_{5}+15 h y_{4} \text {, } \\
& Y_{n-1}=y_{n-1}+(2 n-3) h x y_{n-1}+(n-1)(n-3) h y_{n-2} \text {, } \\
& Y_{n}=y_{n}+(2 n-1) h x y_{n}+n(n-2) h y_{n-1} \text {. }
\end{aligned}
$$

The last of these, for instance, is obtained as follows:

We have $\quad Y_{n}=\frac{d Y_{n-1}}{d X}$.

But

$$
\frac{d}{d X}=\frac{1}{1-2 h x} \cdot \frac{d}{d x}=(1+2 h x) \frac{d}{d x}
$$

and

$$
\begin{aligned}
\frac{d Y_{n-1}}{d x} & =\frac{d}{d x}\left\{y_{n-1}+(2 n-3) h x y_{n-1}+(n-1)(n-3) h y_{n-2}\right\} \\
& =y_{n}+(2 n-3) h x y_{n}+n(n-2) h y_{n-1} .
\end{aligned}
$$


Consequently, $Y_{n}=(1+2 h x) \frac{d Y_{n-1}}{d x}$

$$
\begin{aligned}
& =(1+2 h x)\left\{y_{n}+(2 n-3) h x y_{n}+n(n-2) h y_{n-1}\right\} \\
& =y_{n}+(2 n-1) h x y_{n}+n(n-2) h y_{n-1} .
\end{aligned}
$$

On substituting the above values of $Y, Y_{1}, Y_{2}, \ldots Y_{n}$ in the transformed function, we find immediately

$$
F\left(Y, Y_{1}, Y_{2}, \ldots Y_{n}\right)=(1+h x \nu+h \Theta) F\left(y, y_{1}, y_{2}, \ldots y_{n}\right),
$$

where $\nu$ and $\Theta$ are the partial differential operators

$$
\begin{aligned}
\nu & =-y \partial_{y}+y_{1} \partial_{y_{1}}+3 y_{2} \partial_{y_{2}}+5 y_{3} \partial_{y_{3}}+7 y_{4} \partial_{y_{4}}+\ldots, \\
\Theta & =-y \partial_{y_{1}}+3 y_{2} \partial_{y_{3}}+8 y_{3} \partial_{y_{4}}+15 y_{4} \partial_{y_{5}}+\ldots+n(n-2) y_{n-1} \partial_{y_{n}} .
\end{aligned}
$$

Changing to our usual notation, we write

$$
y_{1}=t, y_{2}=2 a, y_{3}=2.3 b, y_{4}=2.3 .4 c, \ldots,
$$

and then if $F_{1}$ is what $F$ (a rational integral function of $a, b, c, \ldots$ ) becomes when we substitute $\frac{x}{1+h x}, \frac{y}{1+h x}$ for $x, y$ (regarding $h$ as infinitesimal), we have

$$
F_{1}=(1+h x \nu+h \Theta) F,
$$

where

$$
\nu=-y \partial_{y}+t \partial_{t}+3 a \partial_{a}+5 b \partial_{b}+7 c \partial_{c}+9 d \partial_{d}+\ldots,
$$

and

$$
\Theta=-y \partial_{t}+a \partial_{b}+2 b \partial_{c}+3 c \partial_{d}+4 d \partial_{e}+\ldots
$$

In general $\nu$ is merely the partial differential operator written above; but when its subject, $F$, is homogeneous, of degree $i$, and isobaric, of weight $w$, in the letters $y, \quad t, \quad a, \quad b, \quad c, \quad d, \ldots$ supposed to be of degrees $\quad 1,1,1,1,1,1, \ldots$ and of weights $-2,-1,0,1,2,3, \ldots$, its operation is equivalent to multiplication by the number $3 i+2 w$. For in this case we have

$$
y \partial_{y}+t \partial_{t}+a \partial_{a}+b \partial_{b}+c \partial_{c}+d \partial_{d}+\ldots=i
$$

and

$$
-2 y \partial_{y}-t \partial_{t}+b \partial_{b}+2 c \partial_{c}+3 d \partial_{d}+\ldots=w
$$

so that we may regard $\nu$ as a number, simply writing

when we have occasion to do so.

$$
\nu=3 i+2 w
$$

We are now able to show that if $F$ is a persistent form, we must necessarily have

$$
\Theta F=0 .
$$

For

$$
\frac{F_{1}}{F}=1+\nu h x+\frac{h \Theta F}{F}
$$


and consequently, if $F_{1}$ is divisible by $F$ (this is what is meant by saying that $F$ is a persistent form), unless $\Theta F$ vanishes, $\frac{\Theta F}{F}$ must be a rational integral function of $y, t, a, b, c, \ldots$. But since the operation of $\Theta$ diminishes the weight by unity without altering the degree, $\frac{\Theta F}{F}$ must be of degree 0 and weight -1 . The impossibility of the existence of such a function leads to the necessary conclusion that

$$
\Theta F=0 .
$$

Let us apply this result to the case of a pure reciprocant. We have

$$
\Theta=-y \partial_{t}+a \partial_{b}+2 b \partial_{c}+3 c \partial_{d}+\ldots=-y \partial_{t}+\Omega
$$

Thus when $F$ is a pure reciprocant, or indeed any function in which $t$ does not appear, $y \partial_{t} F=0$ and $\Theta$ reduces to $\Omega$. We have therefore shown, in what precedes, that the condition

$$
\Omega F=0
$$

is necessary to ensure the persistence of the form of $F$ under a particular homographic substitution; $d$ fortiori, this condition is also necessarily satisfied when the form of $F$ is persistent under the most general homographic substitution (in which $x, y$ are changed into $\left.\frac{l x+m y+n}{l^{\prime \prime} x+m^{\prime \prime} y+n^{\prime \prime}}, \frac{l^{\prime} x+m^{\prime} y+n^{\prime}}{l^{\prime \prime} x+m^{\prime \prime} y+n^{\prime \prime}}\right)$.

The satisfaction of $\Omega F=0$ is of itself inadequate to ensure persistence under the general homographic substitution; the necessary and sufficient condition of pure reciprocants

$$
V F=0
$$

must also be satisfied. This follows from the fact that the general linear substitution, for which all pure reciprocants are persistent, is merely a particular case of the most general homographic substitution.

It only remains to be proved that the two conditions $V F=0, \Omega F=0$, taken conjointly, are sufficient as well as necessary.

In what follows I use a method which may be termed that of composition of variations. Its nature and value will be better understood if I first apply it to the rigorous demonstration of the theorem that the substitution of $x+h y$ for $x$ in the Quantic

$$
(a, b, c, \ldots \gamma x, y)^{n}
$$

changes any function whatever of its coefficients, say

$$
F(a, b, c, \ldots) \text {, into } e^{h \Omega} F(a, b, c, \ldots) \text {. }
$$


This is not proved, but only verified up to terms of the second order of differentiation, in Salmon's Modern Higher Algebra (3rd ed. 1876, p. 59). Remembering that, whatever the order $n$ of the Quantic may be, the changed values of the coefficients $a, b, c, d, \ldots$ are

$$
\begin{aligned}
& a^{\prime}=a, \\
& b^{\prime}=b+a h, \\
& c^{\prime}=c+2 b h+a h^{2}, \\
& d^{\prime}=d+3 c h+3 b h^{2}+a h^{3},
\end{aligned}
$$

what we have to prove is that, for all values of $h$,

$$
F\left(a^{\prime}, b^{\prime}, c^{\prime}, d^{\prime}, \ldots\right)=e^{h \Omega} F(a, b, c, d, \ldots) .
$$

In other words, if for brevity we write

$$
\begin{aligned}
& F(a, b, c, \ldots)=F, \\
& F\left(a^{\prime}, b^{\prime}, c^{\prime}, \ldots\right)=F_{1},
\end{aligned}
$$

and

it is required to show that

$$
F_{1}=F+h \Omega F+\frac{h^{2}}{1.2} \Omega^{2} F+\frac{h^{3}}{1.2 .3} \Omega^{3} F+\ldots
$$

where

$$
\Omega=a \partial_{b}+2 b \partial_{c}+3 c \partial_{d}+\ldots
$$

When $h$ is infinitesimal, it is obvious that

$$
F_{1}=F+h \Omega F \text {. }
$$

Hence, when $h$ has a general value, we may assume

$$
F_{1}=F+h \Omega F+\frac{h^{2}}{1.2} P+\frac{h^{3}}{1.2 .3} Q+\frac{h^{4}}{1.2 .3 .4} R+\ldots
$$

Let $h$ be increased by the infinitesimal quantity $\epsilon$; then, considering this increase as resulting from a second substitution similar to the first, we see that $F_{1}$ becomes

But it also becomes

$$
F_{1}+\epsilon \Omega F_{1}
$$

$$
\begin{aligned}
F+(h+\epsilon) \Omega F+\frac{(h+\epsilon)^{2}}{1.2} P+\frac{(h+\epsilon)^{3}}{1.2 .3} Q+\ldots=F_{1}+\epsilon \frac{d F_{1}}{d h} \\
=F_{1}+\epsilon\left(\Omega F+h P+\frac{h^{2}}{1.2} Q+\frac{h^{3}}{1.2 .3} R+\ldots\right) .
\end{aligned}
$$

Equating this to $F_{1}+\epsilon \Omega F_{1}$, we obtain

$$
\Omega F_{1}=\Omega F+h P+\frac{h^{2}}{1.2} Q+\frac{h^{3}}{1.2 .3} R+\ldots
$$

But

$$
\Omega F_{1}=\Omega\left(F+h \Omega F+\frac{h^{2}}{1.2} P+\frac{h^{3}}{1.2 .3} Q+\ldots\right) .
$$


The comparison of these two expressions gives

$$
\begin{aligned}
& P=\Omega^{2} F, \\
& Q=\Omega P=\Omega^{3} F, \\
& R=\Omega Q=\Omega^{4} F,
\end{aligned}
$$

Substituting these values in the assumed expansion for $F_{1}$, there results

$$
F_{1}=F+h \Omega F+\frac{h^{2}}{1.2} \Omega^{2} F+\frac{h^{3}}{1.2 .3} \Omega^{3} F+\ldots
$$

which is the expanded form of

$$
F_{1}=e^{h \Omega} F \text {. }
$$

A similar method of procedure will enable us to establish the corresponding but more elaborate formula

$$
F_{1}=(1+h x)^{v} e^{\frac{h \Theta}{1+h x}} F,
$$

in which $F$ is any homogeneous and isobaric function* of degree $i$ and weight $w$ in $y$ and its modified derivatives $(t, a, b, c, \ldots)$ with respect to $x$; the operator $\Theta=-y \partial_{t}+a \partial_{b}+2 b \partial_{c}+3 c \partial_{d}+\ldots$; the function $F_{1}$ is what $F$ becomes in consequence of the substitution of $\frac{x}{1+h x}, \frac{y}{1+h x}$ for $x, y ; h$ is any finite quantity, and $\nu=3 i+2 w$.

Before giving the proof of this theorem, I will show that, upon the assumption of its truth, two inverse finite substitutions will, as they ought, nullify each other, leaving the function operated upon unaltered in form.

To avoid needless periphrasis, we call the substitution of $\frac{x}{1+h x}, \frac{y}{1+h x}$ for $x, y$ the substitution $h$.

Either of the two substitutions, $h,-h$, reverses the effect of the other; for the substitution $-h$ turns

$$
\begin{aligned}
& \frac{x}{1+h x} \text { into } \frac{x}{1-h x} \div 1+\frac{h x}{1-h x}=x, \\
& \frac{y}{1+h x} \text { into } \frac{y}{1-h x} \div 1+\frac{h x}{1-h x}=y .
\end{aligned}
$$

and

The two substitutions $h,-h$, performed successively on $F$, ought therefore to leave its value unaltered. But by hypothesis the substitution $h$ converts $F$ into $F_{1}$; consequently the substitution $-h$ performed on $F_{1}$ ought to change it back again into $F$.

* $F$ need not be integral or even rational; whenever it is homogeneous or isobaric, $\nu$ will be a number. 
It must be carefully observed that (since the operation of $\Theta$ decreases the weight by unity, leaving the degree unchanged) the weight of $\Theta^{\kappa} F$ is $\kappa$ units lower than that of $F$, whilst the degree is the same for both.

Thus for $F$ we have $3 i+2 w=\nu$,

and for

$\Theta^{\kappa} F \quad 3 i+2(w-\kappa)=\nu-2 \kappa$.

Hence the substitution $-h$, which changes

$$
F \text { into }(1-h x)^{\nu} e^{-\frac{h \Theta}{1-h x}} F
$$

also changes

$$
\Theta F \quad, \quad(1-h x)^{\nu-2} e^{-\frac{h \Theta}{1-h x}} \Theta F,
$$$$
\Theta^{2} F \quad, \quad(1-h x)^{\nu-4} e^{-\frac{h \Theta}{1-h x}} \Theta^{2} F,
$$

and in general

$$
\Theta^{\kappa} F \text { into }(1-h x)^{\nu-2 \kappa} e^{-\frac{h \Theta}{1-h x}} \Theta^{\kappa} F \text {. }
$$

Moreover, $1+h x$ becomes $1+\frac{h x}{1-h x}=(1-h x)^{-1}$, so that

$$
\begin{aligned}
(1+h x)^{\nu-\kappa} \Theta^{\kappa} F \text { becomes } & (1-h x)^{-(\nu-\kappa)}(1-h x)^{\nu-2 \kappa} e^{-\frac{h \Theta}{1-h x} \Theta^{\kappa} F} \\
= & (1-h x)^{-\kappa} e^{-\frac{h \Theta}{1-h x}} \Theta^{\kappa} F \\
= & e^{-\frac{h \Theta}{1-h x}}(1-h x)^{-\kappa} \Theta^{\kappa} F(\text { since } \Theta \text { does not act on } x) .
\end{aligned}
$$

Consequently, $(1+h x)^{\nu} F \quad$ becomes $e^{-\frac{h \Theta}{1-h x}} F$,

$$
\begin{array}{lll}
(1+h x)^{\nu-1} \Theta F & \text { " } & e^{-\frac{h \Theta}{1-h . x}}(1-h x)^{-1} \Theta F, \\
(1+h x)^{\nu-2} \Theta^{2} F & \# \quad e^{-\frac{h \Theta}{1-h x}}(1-h x)^{-2} \Theta^{2} F,
\end{array}
$$

And since, by the formula to be verified,

$$
\begin{gathered}
F_{1}=(1+h x)^{\nu} F+h(1+h x)^{\nu-1} \Theta F+\frac{h^{2}}{1.2}(1+h x)^{\nu-2} \Theta^{2} F+\ldots \\
F_{1} \text { becomes } e^{-\frac{h \Theta}{1-h x}}\left\{1+h(1-h x)^{-1} \Theta+\frac{h^{2}}{1.2}(1-h x)^{-2} \Theta^{2}+\ldots\right\} F \\
=e^{-\frac{h \Theta}{1-h x}} e^{\frac{h \Theta}{1-h x}} F=F
\end{gathered}
$$




\section{LECTURE XXIII.}

We now proceed to show how the composition of variations can be made to furnish a strict proof of the formula

$$
F_{1}=(1+h x)^{v} e^{\frac{h \Theta}{1+h x}} F,
$$

which was set forth in the preceding lecture.

As before, calling the change of $x, y$ into $\frac{x}{1+h x}, \frac{y}{1+h x}$, the substitution $h$, it is easy to see that the product of two substitutions, $h, \epsilon$, is the substitution $h+\epsilon$. For

$$
\begin{aligned}
& \frac{x}{1+h x} \div 1+\epsilon \frac{x}{1+h x}=\frac{x}{1+(h+\epsilon) x}, \\
& \frac{y}{1+h x} \div 1+\epsilon \frac{x}{1+h x}=\frac{y}{1+(h+\epsilon) x} .
\end{aligned}
$$

This shows that if

$$
F_{1} \text { is what } F^{\prime} \text { becomes on making the substitution } h \text {, }
$$

and

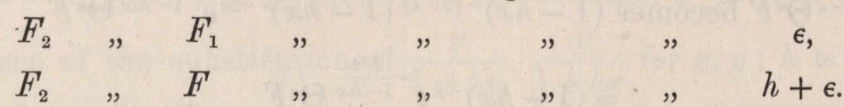

then $F_{2} \quad " F \quad$ " $\quad$ ",$\quad$ " $h+\epsilon$.

Thus we can find two expressions for $F_{2}$, the comparison of which will enable us to assign the coefficients of all the powers of $h$ in the expanded values of $F_{1}$.

The first two terms of this expansion were obtained, in the preceding lecture, by treating $h$ as an infinitesimal. We may therefore write

$$
F_{1}=F+h(\nu x+\Theta) F+\frac{h^{2}}{1.2} N_{2}+\frac{h^{3}}{1.2 .3} N_{3}+\ldots
$$

Changing $h$ into $h+\epsilon$, we deduce

$$
F_{2}=F+(h+\epsilon)(\nu x+\Theta) F+\frac{(h+\epsilon)^{2}}{1.2} N_{2}+\frac{(h+\epsilon)^{3}}{1.2 .3} N_{3}+\ldots
$$

For greater simplicity, let $\epsilon$ be an infinitesimal, and write

$$
\frac{F_{2}-F_{1}}{\epsilon}=\Delta F_{1}
$$

Then

$$
\Delta F_{1}=(\nu x+\Theta) F+h N_{2}+\frac{h^{2}}{1.2} N_{3}+\ldots
$$

Now look at each term in the expansion of $F_{1}$ and find its increment (that is, its $\Delta$ ) when $x, y$ undergo the substitution $\epsilon$. We thus obtain

$$
\Delta F_{1}=\Delta F+h \Delta(\nu x+\Theta) F+\frac{h^{2}}{1.2} \Delta N_{2}+\frac{h^{3}}{1.2 .3} \Delta N_{3}+\ldots
$$


Comparing these two values of $\Delta F_{1}$, we find

and generally

$$
\begin{aligned}
& N_{2}=\Delta(\nu x+\Theta) F, \\
& N_{3}=\Delta N_{2}, \\
& N_{4}=\Delta N_{3}, \\
& \cdots \cdots \cdots \cdots \\
& N_{r}=\Delta N_{r-1} .
\end{aligned}
$$

These equations are sufficient to determine all the coefficients of $F_{1}$; it only remains to show how the operations $\Delta$ may be performed.

We have in fact

where

$$
F_{1}=F+h \Delta F+\frac{h^{2}}{1.2} \Delta^{2} F+\frac{h^{3}}{1.2 .3} \Delta^{3} F+\ldots
$$

But we must not from this rashly infer that

$$
\Delta^{n} F=(\nu x+\Theta)^{n} F \text {. }
$$

To do so would be tantamount to regarding $\nu$ as a constant number, whereas its value depends on the degree and weight of the subject of operation.

This will be clearly seen in the calculation which follows*. We first generalize the formula

$$
\Delta F^{\prime}=(\nu x+\Theta) F
$$

by making $\Theta^{\kappa} F$ the operand instead of $F$.

Then, since $i$ is the degree and $w-\kappa$ the weight of $\Theta^{\kappa} F$, instead of

$$
3 i+2 w=\nu,
$$

we have

$$
3 i+2(w-\kappa)=\nu-2 \kappa \text {. }
$$

Thus,

$$
\Delta \Theta^{\kappa} F^{\prime}=\{(\nu-2 \kappa) x+\Theta\} \Theta^{\kappa} F \text {. }
$$

Again, since

$$
\Delta x=\left(\frac{x}{1+\epsilon x}-x\right) \div \epsilon=-x^{2} \text {, }
$$

we find

$$
\Delta x^{\lambda} \Theta^{\kappa} F=\lambda x^{\lambda-1} \Theta^{\kappa} F . \Delta x+x^{\lambda} \Delta \Theta^{\kappa} F=-\lambda x^{\lambda+1} \Theta^{\kappa} F^{\prime}+x^{\lambda}\left\{(\nu-2 \kappa) x+\Theta^{\prime}\right\} \Theta^{\kappa} F .
$$

Hence we obtain the general formula

$$
\Delta x^{\lambda} \Theta^{\kappa} F=x^{\lambda}\{(\nu-2 \kappa-\lambda) x+\Theta\} \Theta^{\kappa} F,
$$

\footnotetext{
* If our sole object were to show that $\Theta F=0$ is a sufficient as well as necessary condition of the persistence of $F$, we might dispense with all further calculation. Thus it is obvious that, since $\Delta F=(\nu x+\Theta) F, \Delta^{n} F$ must be of the form $(x, \Theta)^{n} F$; for the dependence of $\nu$ on the degreeweight of the operand will not affect the form of $\Delta^{n}$, but only its numerical coefficients. Hence we conclude that $F_{1}$ is of the form $\phi(x, \theta) F$; and remembering that $\theta^{2} F=0, \theta^{3} F=0$, ... whenever $\theta F=0$, it is at once seen that not only (as was shown in the last lecture) must $\theta F$ vanish when $F$ is persistent under the substitution $h$, but, conversely, that when $\Theta F=0$, the altered value of $F$ contains the original value as a factor (the other factor being in this case a function of $x$ onily); that is, $F$ is persistent.
}

S IV. 
by means of which we calculate in succession the values of $\Delta^{2} F, \Delta^{3} F, \ldots$. Thus,

Hence

$$
\begin{aligned}
\Delta^{2} F^{\prime} & =\Delta(\nu x+\Theta) F \\
& =\nu \Delta x F^{\prime}+\Delta \Theta F \\
& =\nu x\{(\nu-1) x+\Theta\} F+\{(\nu-2) x+\Theta\} \Theta F \\
& =\left\{\nu(\nu-1) x^{2}+2(\nu-1) x \Theta+\Theta^{2}\right\} F .
\end{aligned}
$$

$\Delta^{3} F=\nu(\nu-1) \Delta x^{2} F+2(\nu-1) \Delta x \Theta F+\Delta \Theta^{2} F$

$$
\begin{aligned}
& =\nu(\nu-1) x^{2}\{(\nu-2) x+\Theta\} F+2(\nu-1) x\{(\nu-3) x+\Theta\} \Theta F \\
& +\quad\{(\nu-4) x+\Theta\} \Theta^{2} F \\
& =\left\{\nu(\nu-1)(\nu-2) x^{3}+3(\nu-1)(\nu-2) x^{2} \Theta+3(\nu-2) x \Theta^{2}+\Theta^{3}\right\} F .
\end{aligned}
$$

If $[\nu]^{n}$ is used to denote $\nu(\nu-1)(\nu-2) \ldots$ to $n$ factors $\left([\nu]^{1}\right.$ will of course mean $\nu$ ), we have shown that

$$
\begin{aligned}
\Delta F & =\left([\nu]^{1} x+\Theta\right) F \\
\Delta^{2} F & =\left([\nu]^{2} x^{2}+2[\nu-1]^{1} x \Theta+\Theta^{2}\right) F, \\
\Delta^{3} F & =\left([\nu]^{3} x^{3}+3[\nu-1]^{2} x^{2} \Theta+3[\nu-1]^{1} x \Theta^{2}+\Theta^{3}\right) F,
\end{aligned}
$$

and by induction it may be proved that in general

$$
\left.\Delta^{n} F=\left\{[\nu]^{n} x^{n}+n[\nu-1]^{n-1} x^{n-1} \Theta+\frac{n(n-1)}{1.2}[\nu-2]^{n-2} x^{n-2} \Theta^{2}+\ldots+\Theta\right)^{n}\right\} F
$$

That the last term of this expression is $\Theta^{n} \boldsymbol{F}$ is sufficiently obvious; what we wish to prove is that, when $m$ is any positive integer less than $n$, the term in $\Delta^{n} F$ which involves $\Theta^{m}$ will be

$$
\frac{n(n-1) \ldots(n-m+1)}{1.2 .3 \ldots m}[\nu-m]^{n-m} x^{n-m} \Theta^{m} F .
$$

To find the term involving $\Theta^{m}$ in $\Delta^{n+1} F$, we need only consider the operation of $\Delta$ on two consecutive terms of $\Delta^{n} F$; none of the remaining terms wili affect the result. Suppose, then, that

$$
\Delta^{n} F=\ldots+p x^{n-m} \Theta^{m} F+q x^{n-m+1} \Theta^{m-1} F+\ldots
$$

Operating with $\Delta$, we find

$$
\begin{aligned}
\Delta^{n+1} F= & \ldots+p \Delta x^{n-m} \Theta^{m} F+q \Delta x^{n-m+1} \Theta^{m-1} F+\ldots \\
= & \ldots+p x^{n-m}\{(\nu-n-m) x+\Theta\} \Theta^{m} F \\
& +q x^{n-m+1}\{(\nu-n-m+1) x+\Theta\} \Theta^{m-1} F+\ldots \\
= & \ldots+\{p(\nu-n-m)+q\} x^{n+1-m} \Theta^{m} F+\ldots .
\end{aligned}
$$

Now, assuming the general term of $\Delta^{n} F$ to be as written above, we have

$$
\begin{aligned}
& p=\frac{n(n-1) \ldots(n-m+1)}{1.2 .3 \ldots m}[\nu-m]^{n-m}, \\
& q=\frac{n(n-1) \ldots(n-m+2)}{1.2 .3 \ldots(m-1)}[\nu-m+1]^{n-m+1}
\end{aligned}
$$

so that

$$
q=p\left\{\frac{m(\nu-m+1)}{n-m+1}\right\} \text {. }
$$


Thus the general term of $\Delta^{n+1} F$ has for its numerical coefficient

$$
\begin{aligned}
p(\nu-n-m)+q & =p\left\{\frac{m(\nu-m+1)+(\nu-n-m)(n-m+1)}{n-m+1}\right\} \\
& =p\left\{\frac{(n+1)(\nu-n)}{n-m+1}\right\}=\frac{(n+1) n \ldots(n-m+2)}{1.2 .3 \ldots m}[\nu-m]^{n+1-m},
\end{aligned}
$$

which shows that the numerical coefficients in $\Delta^{n+1} F$ obey the same law as those in $\Delta^{n} F$; and as this law is true for $n=1,2,3$, it is also true universally.

We have thus shown that the general term in $\Delta^{n} F$ is

$$
\frac{n(n-1) \ldots(n-m+1)}{1.2 .3 \ldots m}[\nu-m]^{n-m} x^{n-m} \Theta^{m} F,
$$

and, consequently, the corresponding general term in

$$
\frac{h^{n} \Delta^{n} F}{1.2 .3 \ldots n} \text { is } \frac{[\nu-m]^{n-m}}{1.2 .3 \ldots(n-m)} h^{n-m} x^{n-m} \cdot \frac{h^{m} \Theta^{m} F}{1.2 .3 \ldots m} .
$$

Now, as we have already seen,

$$
F_{1}=\left(1+h \Delta+\frac{h^{2}}{1.2} \Delta^{2}+\frac{h^{3}}{1.2 .3} \Delta^{3}+\ldots\right) F
$$

which, by merely expressing the symbolic factor as a series of powers of $\Theta$, may be transformed into

$$
\begin{aligned}
& F_{1}=\left(1+[\nu]^{1} h x+\frac{[\nu]^{2}}{1.2} h^{2} x^{2}+\frac{[\nu]^{3}}{1.2 .3} h^{3} x^{3}+\ldots\right) F \\
& +\left(1+[\nu-1]^{1} h x+\frac{[\nu-1]^{2}}{1.2} h^{2} x^{2}+\frac{[\nu-1]^{3}}{1.2 .3} h^{3} x^{3}+\ldots\right) h \Theta F \\
& +\left(1+[\nu-2]^{1} h x+\frac{[\nu-2]^{2}}{1.2} h^{2} x^{2}+\frac{[\nu-2]^{3}}{1.2 .3} h^{3} x^{3}+\ldots\right) \frac{h^{2} \Theta^{2} F}{1.2}
\end{aligned}
$$

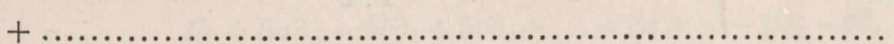

where, remembering that $[\nu]^{n}$ stands for $\nu(\nu-1)(\nu-2) \ldots$ to $n$ factors, it is evident that the functions of $x$ which multiply $F, h \Theta F, \frac{h^{2}}{1.2} \Theta^{2} F, \ldots$ are all of them binomial expansions. Hence we immediately obtain

$$
\begin{aligned}
F_{1} & =(1+h x)^{\nu} F+(1+h x)^{\nu-1} h \Theta F+(1+h x)^{\nu-2} \frac{h^{2}}{1.2} \Theta^{2} F+\ldots \\
& =(1+h x)^{\nu}\left\{1+(1+h x)^{-1} h \Theta+(1+h x)^{-2} \frac{h^{2} \Theta^{2} F}{1.2}+\ldots\right\} F
\end{aligned}
$$

and finally,

$$
F_{1}=(1+h x)^{\nu} e^{\frac{h \Theta}{1+h x}} F .
$$

Mr Hammond has remarked that, with a slight modification, the foregoing demonstration will serve to establish the analogous theorem, that

$$
F_{1}=(1+h t)^{-\mu} e^{-\frac{h V_{1}}{1+h t}} F
$$


where, as before, $F$ means any homogeneous and isobaric function of degree $i$ and weight $w$ in the letters $y, t, a, b, c, \ldots$; and $F_{1}$ is what $F$ becomes when, leaving $y$ unaltered, we change $x$ into $x+h y$, where $h$ is any finite quantity. Instead of the operator

$$
\Theta=-y \partial_{t}+a \partial_{b}+2 b \partial_{c}+3 c \partial_{d}+\ldots=-y \partial_{t}+\Omega
$$

we have $\quad-V_{1}=y t \partial_{y}+t^{2} \partial_{t}-2 a^{2} \partial_{b}-5 a b \partial_{c}-\ldots=y t \partial_{y}+t^{2} \partial_{t}-V^{*}$;

and instead of $\nu=3 i+2 w$, a different number, $\mu=3 i+w$ (which I have called the characteristic), taken negatively.

If we suppose that

$$
F_{1} \text { is what } F \text { becomes on changing } x \text { into } x+h y \text {, }
$$

and $F_{2} \quad " \quad F \quad " \quad, \quad$ " $\quad x \quad, \quad x+\epsilon y$,

then $F_{2} \quad, \quad F \quad$ " $\quad$ " $\quad x \quad$, $\quad x+(h+\epsilon) y$.

Hence, if

$$
F_{1}=F+h P+\frac{h^{2}}{1.2} Q+\frac{h^{3}}{1.2 .3} R+\ldots
$$

we must have

$$
\begin{aligned}
F_{2} & =F+(h+\epsilon) P+\frac{(h+\epsilon)^{2}}{1.2} Q+\frac{(h+\epsilon)^{3}}{1.2 .3} R+\ldots \\
& =F_{1}+\epsilon \frac{d F_{1}}{d h}+\ldots
\end{aligned}
$$

Thus, if $\epsilon$ be regarded as infinitesimal, and we write

it follows that

$$
\begin{gathered}
\frac{F_{2}-F_{1}}{\epsilon}=\Delta F_{1} \\
\Delta F_{1}=P+h Q+\frac{h^{2}}{1.2} R+\ldots .
\end{gathered}
$$

But, by the direct operation of $\Delta$, we find

$$
\Delta F_{1}=\Delta F+h \Delta P+\frac{h^{2}}{1.2} \Delta Q+\ldots
$$

and, comparing these two values of $\Delta F_{1}$,

$$
\begin{aligned}
& P=\Delta F, \\
& Q=\Delta P=\Delta^{2} F, \\
& R=\Delta Q=\Delta^{3} F,
\end{aligned}
$$

Hence it follows that

$$
F_{1}=F+h \Delta F+\frac{h^{2}}{1.2} \Delta^{2} F+\frac{h^{3}}{1.2 .3} \Delta^{3} F+\ldots
$$

* This theorem was stated without proof in Lecture VIII, where, through inadvertence, the term $y t \partial_{y}$ in the expression for $V_{1}$ was omitted [p. 352, above]. 
It remains to find the value of $\Delta^{n} F$. This can be effected by means of formulae given in Lecture VIII. [p. 350, above], where it is shown that

$$
\begin{aligned}
& \Delta x=y \\
& \Delta y=0 \\
& \Delta t=-t^{2} \\
& \Delta a=-3 a t \\
& \Delta b=-4 b t-2 a^{2}, \\
& \Delta c=-5 c t-5 a b \\
& \Delta d=-6 d t-6 a c-3 b^{2}, \\
& \Delta e=-7 e t-7 a d-7 b c
\end{aligned}
$$

We now show that

$$
\Delta F=-\left(\mu t+V_{1}\right) F
$$

where

$$
V_{1}=V-t^{2} \partial_{t}-y t \partial_{y}
$$

just as in the cognate theorem we had

$$
\Delta F=(\nu x+\Theta) F
$$

Since $F$ is a function of $y, t, a, b, c, \ldots$ without $x$, it is evident that

$$
\begin{aligned}
\Delta F^{\prime}= & \frac{d F}{d y} \Delta y+\frac{d F}{d t} \Delta t+\ldots \\
= & -t\left(t \partial_{t}+3 a \partial_{a}+4 b \partial_{b}+5 c \partial_{c}+\ldots\right) F \\
& -\left\{2 a^{2} \partial_{b}+5 a b \partial_{c}+\left(6 a c+3 b^{2}\right) \partial_{d}+\ldots\right\} F,
\end{aligned}
$$

where the part of $\Delta F$ which is independent of $t$ is $-V F$.

Now, $\quad y \partial_{y}+t \partial_{t}+a \partial_{a}+b \partial_{b}+c \partial_{c}+\ldots=i$

$$
\begin{array}{lrl}
\text { and } & -2 y \partial_{y}-t \partial_{t} \quad+b \partial_{b}+2 c \partial_{c}+\ldots & =w ; \\
\text { so that } & t \partial_{t}+3 a \partial_{a}+4 b \partial_{b}+5 c \partial_{c}+\ldots=3 i+w-y \partial_{y}-t \partial_{t} .
\end{array}
$$

Hence, writing $3 i+w=\mu$,

$$
\begin{aligned}
\Delta F & =-t\left(\mu-y \partial_{y}-t \partial_{t}\right) F-V F \\
& =-\left(\mu t+V_{1}\right) F \\
V_{1} & =V-t^{2} \partial_{t}-y t \partial_{y} .
\end{aligned}
$$

where

Observing that $V_{1}{ }^{\kappa} F$ is of degree $i+\kappa$ and weight $w-\kappa$; since

$$
3(i+\kappa)+(w-\kappa)=\mu+2 \kappa,
$$

we see that

$$
\Delta V_{1}{ }^{\kappa} F=-\left\{(\mu+2 \kappa) t+V_{1}\right\} V_{1}{ }^{\kappa} F \text {. }
$$

Again,

$$
\begin{aligned}
\Delta t^{\lambda} V_{1}{ }^{\kappa} F & =\lambda t^{\lambda-1} V_{1}{ }^{\kappa} F \cdot \Delta t+t^{\lambda} \Delta V_{1}{ }^{\kappa} F \\
& =-\lambda t^{\lambda+1} V_{1}{ }^{\kappa} F-t^{\lambda}\left\{(\mu+2 \kappa) t+V_{1}\right\} V_{1}{ }^{\kappa} F .
\end{aligned}
$$

We thus obtain the formula

$$
\Delta t^{\lambda} V_{1}^{\kappa} F=-t^{\lambda}\left\{(\mu+\lambda+2 \kappa) t+V_{1}\right\} V_{1}^{\kappa} F^{\prime}
$$

analogous to the one previously employed,

$$
\Delta x^{\lambda} \Theta^{\kappa} F=x^{\lambda}\{(\nu-2 \kappa-\lambda) x+\Theta\} \Theta^{\kappa} F .
$$


The remainder of the work will be step for step the same for this as for the previous theorem. In fact, by using (1) just as we used (2), we shall deduce

$$
F_{1}=(1+h t)^{-\mu} e^{-\frac{h V_{1}}{1+h t}} F
$$

just as we deduced the analogous formula

$$
F_{1}=(1+h x)^{\nu} e^{\frac{h \Theta}{1+h x}} F \text {. }
$$

The reason of this is obvious: by interchanging $x$ and $t, \mu$ and $-\nu, \Theta$ and $-V_{1}$, we interchange the formulae (1) and (2), (3) and (4).

It may be well to observe that if we use $S_{h}$ to denote a substitution of such a nature that

$$
S_{\epsilon} S_{h}=S_{h+\epsilon}
$$

and if (regarding $\epsilon$ as an infinitesimal) we write

$$
\frac{S_{\epsilon}-1}{\epsilon}=\Delta
$$

then in general

$$
S_{h} F=e^{h \Delta} F .
$$

The proof of this proposition is virtually contained in what precedes.

\section{LECTURE XXIV.}

Whenever a rational integral function of $x, y, t, a, b, c, \ldots$ is persistent in form under the general linear substitution, it cannot contain explicitly either $x, y$ or $t$, but must be a function of the remaining letters $a, b, c, \ldots$ (the successive modified derivatives, beginning with the second, of $y$ with respect to $x$ ) alone.

For if, keeping $y$ unaltered, we change $x$ into $x+\alpha$, where $\alpha$ is any arbitrary constant which may be regarded as an infinitesimal, the derivatives $t, a, b, c, \ldots$ are not affected by this change, and consequently the function

$$
F=F(x, y, t, a, b, c, \ldots) \text { becomes } F+\alpha \frac{d F}{d x},
$$

which cannot be divisible by $F$ unless $\frac{d F}{d x}=0$.

(The alternative hypothesis of $\frac{d F}{d x}$ being divisible by $F$ is inadmissible, because $F$ is a rational integral function.)

Hence $F$ cannot contain $x$ explicitly; and if we write $y+\beta$ for $y$, keeping $x$ unchanged, we see, in like manner, that $F$ cannot contain $y$ explicitly. 
Again, if in the function

$$
F=F(t, a, b, c, \ldots)
$$

we change $x, y$ into $x+\alpha, y+\beta, x+\beta$, the effect of this substitution will be to increase $t$ by the arbitrary constant $\beta$, without altering any of the remaining derivatives $a, b, c, \ldots$.

Hence, in order that the form of $F$ may still be persistent, we must have $\frac{d F}{d t}=0$; the reasoning being just the same as that by which $\frac{d F}{d x}$ was seen to vanish. Thus, $F$ does not contain $t$ explicitly. Moreover, the function

$$
F=F(a, b, c, \ldots)
$$

must be both homogeneous and isobaric.

For the substitution of $\alpha, x+\alpha, \beta, y+\beta, x+\beta$ for $x, y$, respectively, will multiply the letters

by

$$
\begin{aligned}
& a, b, \quad c, \quad d, \ldots \\
& \beta_{11} \alpha_{1}^{-2}, \quad \beta_{11} \alpha_{1}^{-3}, \quad \beta_{11} \alpha_{1}^{-4}, \quad \beta_{11} \alpha_{1}^{-5}, \ldots
\end{aligned}
$$

Each term of $F$ will therefore be multiplied by a positive power of $\beta_{\text {, }}$ and a negative power of $\alpha$.

Let one of the terms of $F$ be $a^{\lambda_{0}} b^{\lambda_{1}} c^{\lambda_{2}} d^{\lambda_{3}} \ldots$ It will be multiplied by

$$
\beta_{1,} \lambda_{0}+\lambda_{1}+\lambda_{2}+\lambda_{3}+\ldots \alpha_{1}^{-}\left(2 \lambda_{0}+3 \lambda_{1}+4 \lambda_{2}+5 \lambda_{3}+\ldots\right) \text {. }
$$

In order that $F$ may retain its form, this multiplier must be the same for every term of $F$, no matter what arbitrary values are assigned to $\alpha_{\text {, and }} \beta_{1}$. This can only happen when, for all terms of the function $F$, we have

and

$$
\lambda_{0}+\lambda_{1}+\lambda_{2}+\lambda_{3}+\ldots=\text { const. }
$$

that is, when $F$ is homogeneous and isobaric.

We have thus proved that among all the rational integral functions of $x, y, t, a, b, c, \ldots$ the only ones persistent under the substitution of $\alpha+\alpha, x$, $\beta+\beta, x+\beta_{1} y$ for $x, y$, respectively, are such as simultaneously satisfy the conditions of not explicitly containing $x, y$ or $t$, and of being homogeneous and isobaric in the remaining letters $a, b, c, \ldots$.

If $F$, any function satisfying these conditions, merely acquires an extraneous factor when, leaving $y$ unaltered, we change $x$ into $x+h y$, the form of $F$ will be persistent under the general linear substitution. For both $\alpha+\alpha,(x+h y)$ and $\beta+\beta_{,}(x+h y)+\beta_{1} y$ are general linear functions of $x, y, 1$.

Now, the change of $x$ into $x+h y$ converts (as was shown in the preceding lecture) $F$ into

where

$$
\begin{aligned}
& F_{1}=(1+h t)^{-\mu} e^{-\frac{h V_{1}}{1+h t}} F \\
& V_{1}=V-t^{2} \partial_{t}-y t \partial_{y}
\end{aligned}
$$


But, since neither $y$ nor $t$ occurs in $F$, we must have

Consequently,

$$
\begin{aligned}
& \partial_{y} F=0 \text { and } \partial_{t} F=0 . \\
& V_{1} F=V F, \quad V_{1}^{2} F=V^{2} F,
\end{aligned}
$$

and so on. Hence

$$
\begin{aligned}
F_{1}^{\prime} & =(1+h t)^{-\mu} e^{-\frac{h V}{1+h t}} F \\
& =(1+h t)^{-\mu} F-(1+h t)^{-\mu-1} h V F+(1+h t)^{-\mu-2} \frac{h^{2} V^{2}}{1.2} F-\ldots
\end{aligned}
$$

Unless $V F, V^{2} F, V^{3} F, \ldots$ all of them vanish, $F_{1}$ cannot contain $F$ as a factor. If it could, $V F, V^{2} F, \ldots$ would all have to be divisible by $F$. But this is impossible; for $V F$, a rational integral function of $a, b, c, \ldots$ whose weight is $w-1$, cannot be divisible by $F$, a rational integral function of weight $w$.

We must therefore have

$$
V F=0
$$

(which implies $V^{2} F=0$, etc.) as the necessary and sufficient condition of the persistence of the form of $F$ under the general linear substitution. In other words, $F$ must be a pure reciprocant.

In order that $F$ may also be persistent in form under the general homographic substitution, it must (besides being a pure reciprocant) be subject to annihilation by the operator

$$
\Omega=a \partial_{b}+2 b \partial_{c}+3 c \partial_{d}+\ldots
$$

For it was seen, in the preceding lecture, that the special homographic substitution in which $\frac{x}{1+h x}, \frac{y}{1+h x}$ are written instead of $x, y$, respectively, has the effect of changing any homogeneous and isobaric function $F$ into $F_{1}$, where

$$
\begin{aligned}
& F_{1}=(1+h x)^{\nu} e^{\frac{h \Theta}{1+h x}} F, \\
& \Theta=\Omega-y \partial_{t} .
\end{aligned}
$$

When the letter $t$ does not occur in $F$, we may write $\partial_{t} F=0$, so that $\Theta$ becomes simply $\Omega$, and the above formula becomes

$$
F_{1}=(1+h x)^{\nu} e^{\frac{h \Omega}{1+h x}} F \text {. }
$$

Hence it follows immediately that, when $F$ is a rational integral function of the letters $a, b, c, \ldots$, the condition $\Omega F=0$ is sufficient as well as necessary to ensure the persistence of the form of $F$ under the special homographic substitution we have employed.

But when $F$ is a pure reciprocant it also satisfies the condition $V F=0$, and it is the simultaneous satisfaction of $\Omega F=0$ and $V F=0$ that ensures 
the persistence of the form of $F$ under the most general homographic substitution. This may be shown by combining the substitution $\frac{x}{1+h x}, \frac{y}{1+h x}$ (for which $F$ is persistent when, and only when, $\Omega F^{\prime}=0$ ) with the general linear substitution (for which $V F=0$ is the necessary and sufficient condition of the persistence of the form of $F$ ), so as to obtain the most general homographic substitution. Thus the linear substitution

when combined with

$$
\left.\begin{array}{l}
x=l x_{1}+m y_{1}+n \\
y=l^{\prime} x_{1}+m^{\prime} y_{1}+n^{\prime}
\end{array}\right\}
$$

gives the substitution

$$
x_{1}=\frac{x_{11}}{1+h x_{1 \prime}}, y_{1}=\frac{y_{11}}{1+h x_{1 \prime}}
$$

$$
\left.\begin{array}{l}
x=\frac{l x_{11}+m y_{11}+n\left(1+h x_{11}\right)}{1+h x_{1 \prime}} \\
y=\frac{l^{\prime} x_{11}+m^{\prime} y_{11}+n^{\prime}\left(1+h x_{1 \prime}\right)}{1+h x_{\prime \prime}}
\end{array}\right\},
$$

in which both the numerators are general linear functions.

By combining the substitution just obtained with the linear substitution

$$
x_{1 \prime}=\lambda x_{1 \prime}+\mu y_{1 \prime}+\nu, y_{1 \prime}=y_{1 \prime \prime}
$$

the denominator of each fraction is changed into a general linear function, and thus, by combining the special homographic substitution $\frac{x}{1+h x}, \frac{y}{1+h x}$ with two linear substitutions, we arrive at the most general homographic substitution.

This proves that the necessary and sufficient condition of $F$ being a homographically persistent form is the coexistence of the two conditions

$$
V F=0, \Omega F=0 .
$$

Thus a Projective Reciprocant, or Principiant, or Differential Invariant, combines the natures of a Pure Reciprocant and Invariant in respect of the elements.

Notice that every Pure Reciprocant is an Invariant of the Reciprocal Function (that is, the numerator of the expression for $\frac{d^{n} x}{d y^{n}}$ in terms of $\frac{d y}{d x}$, $\frac{d^{2} y}{d x^{2}}, \ldots$, or what is the same in terms of the modified derivatives $\left.t, a, b, \ldots\right)$, but the elements of such invariants are not the original simple elements, but more or less complicated functions of them.

What has just been stated is obvious from the fact that all invariants of the "reciprocal function" have been shown to be pure reciprocants (vide* Lecture XIX.). The ordinary protomorph invariants of this function will 
have for their leading term a power of $a$ multiplied by a single letter. Consequently, by reasoning previously employed in these lectures, every pure reciprocant will be a rational function of invariants of the Reciprocal Function divided by some power of $a$. Thus, for example, the Reciprocal Function

if

$$
14 a^{4}-21 a^{2} b t+3\left(2 a c+b^{2}\right) t^{2}-d t^{3}=(\alpha, \beta, \gamma, \delta \gamma 1,-t)^{3}
$$

$$
\alpha=14 a^{4}, \beta=7 a^{2} b, \gamma=2 a c+b^{2}, \delta=d .
$$

The two protomorph invariants of this reciprocal function are

$$
\alpha \gamma-\beta^{2}=7 a^{4}\left(4 a c-5 b^{2}\right)
$$

and

$$
\alpha^{2} \delta-3 \alpha \beta \gamma+2 \beta^{3}=196 a^{6}\left(a^{2} d-3 a b c+2 b^{3}\right) .
$$

All other pure reciprocants of extent 3 may be rationally expressed in terms of $a$ and the two protomorphs $4 a c-5 b^{2}, a^{2} d-3 a b c+2 b^{3}$; that is, all pure reciprocants of extent 3 are invariants of the reciprocal function of extent 3 .

The reasoning employed can be applied with equal facility to the general case of extent $n$.

Instead of $\frac{x}{1+h x}, \frac{y}{1+h x}$, let us consider the special homographic substitution $\frac{1}{x}, \frac{y}{x}$ employed by M. Halphen.

$$
\text { Writing } \quad X=\frac{1}{x} \text { and } Y=\frac{y}{x} \text {, }
$$

let $Y_{1}, Y_{2}, Y_{3}, \ldots$ denote the successive derivatives of $Y$ with respect to $X$, and $y_{1}, y_{2}, y_{3}, \ldots$ those of $y$ with respect to $x$. Then

$$
\begin{aligned}
& Y=x^{-1} y \\
& Y_{1}=-x\left(y_{1}-\frac{1}{x} y\right) \\
& Y_{2}=x^{3} y_{2} \\
& Y_{3}=-x^{5}\left(y_{3}+\frac{3}{x} y_{2}\right) \\
& Y_{4}=x^{7}\left(y_{4}+\frac{8}{x} y_{3}+\frac{12}{x^{2}} y_{2}\right) \\
& Y_{5}=-x^{9}\left(y_{5}+\frac{15}{x} y_{4}+\frac{60}{x^{2}} y_{3}+\frac{60}{x^{3}} y_{2}\right)
\end{aligned}
$$

Hence, if $a, b, c, d, \ldots$ are the successive modified derivatives (beginning with the second) of $y$ with respect to $x$, and $a^{\prime}, b^{\prime}, c^{\prime}, d^{\prime}, \ldots$ the corresponding 
modified derivatives of $Y$ with respect to $X$, it follows immediately that

$$
\begin{aligned}
& a^{\prime}=x^{3} a \\
& b^{\prime}=-x^{5}\left(b+\frac{1}{x} a\right) \\
& c^{\prime}=x^{7}\left(c+\frac{2}{x} b+\frac{1}{x^{2}} a\right) \\
& d^{\prime}=-x^{9}\left(d+\frac{3}{x} c+\frac{3}{x^{2}} b+\frac{1}{x^{3}} a\right)
\end{aligned}
$$

Attributing the weights $0,1,2,3, \ldots$ to the letters $a, b, c, d, \ldots$, it is very easily seen that if $F$ is any homogeneous and isobaric function of degree $i$ and weight $w$,

$$
F\left(a^{\prime}, b^{\prime}, c^{\prime}, \ldots\right)=(-)^{w} x^{3 i+2 w} F\left(a, b+\frac{1}{x} a, c+\frac{2}{x} b+\frac{1}{x^{2}} a, \ldots\right) .
$$

But we proved (in Lecture XXII.) [above, p. 429] that for all values of $h$

$$
F\left(a, b+a h, c+2 b h+a h^{2}, \ldots\right)=e^{h \Omega} F(a, b, c, \ldots) .
$$

Hence, making $h=\frac{1}{x}$, we obtain

$$
F^{\prime}\left(a^{\prime}, b^{\prime}, c^{\prime}, d^{\prime}, \ldots\right)=(-)^{w} x^{3 i+2 w} e^{\frac{\Omega}{x}} F^{\prime}(a, b, c, \ldots),
$$

which proves that the satisfaction of

$$
\Omega F(a, b, c, \ldots)=0
$$

is the necessary and sufficient condition for the persistence of the form of $F$ under the Halphenian substitution $\frac{1}{x}, \frac{y}{x}$.

Similarly we might prove that $F(y, t, a, b, c, \ldots)$, which contains $y$ and $t$, but not $x$, is changed by the substitution $\frac{1}{x}, \frac{y}{x}$ into

where

$$
(-)^{w} x^{\nu} e^{\frac{\Theta}{x}} F(y, t, a, b, c, \ldots)
$$

$$
\Theta=-y \partial_{t}+a \partial_{b}+2 b \partial_{c}+\ldots=\Omega-y \partial_{t}
$$

or we may deduce this result from the formula, demonstrated in the preceding lecture of this course,

$$
F_{1}=(1+h x)^{v} e^{\frac{h \Theta}{1+h x}} F
$$

in which $F_{1}$ is what $F$ becomes in consequence of the substitution $\frac{x}{1+h x}$, $\frac{y}{1+h x}$ impressed on the variables. 
Let $i$ be the degree and $\omega$ the weight measured by the sum of the orders of differentiation in each term of

$$
F(y, t, a, b, c, \ldots) \text {. }
$$

If we measure the weight by the sum of the orders of differentiation of every term of $F$ diminished by 2 units for each letter in the term, then

$$
w=\omega-2 i \text { and } 2 \omega-i=3 i+2 w=\nu .
$$

Let

$$
F(y, t, a, b, c, \ldots) \text { become } F^{\prime \prime}(y, t, a, b, c, \ldots),
$$

when we change

$$
x \text { into } q x+p \text { and } y \text { into } r y
$$

then

$$
F^{\prime}(y, t, a, b, c, \therefore)=r^{i} q^{-\omega} F(y, t, a, b, c, \ldots) \text {. }
$$

A further substitution $\frac{x}{1+h x}, \frac{y}{1+h x}$, impressed on the variables in $F^{\prime}$, will convert the original variables into

that is, into

$$
\begin{gathered}
\frac{q x}{1+h x}+p \text { and } \frac{r y}{1+h x}, \\
\frac{p(1+h x)+q x}{1+h x} \text { and } \frac{r y}{1+h x}
\end{gathered}
$$

The function $F^{\prime}$ is at the same time changed into

$$
r^{i} q^{-\omega}(1+h x)^{v} e^{\frac{h \Theta}{1+h x}} F(y, t, a, b, c, \ldots) .
$$

If now, in the above, we write $p=h, q=-h^{2}, r=h$, we shall have changed the original variables $x, y$ into $\frac{h}{1+h x}, \frac{h y}{1+h x}$, and the original function $F$ into $h^{i}\left(-h^{2}\right)^{-\omega}(1+h x)^{v} e^{\frac{h \Theta}{1+h x}} F=(-)^{\omega} h^{i-2 \omega}(1+h x)^{\nu} e^{\frac{h \Theta}{1+h x}} F=(-)^{w}\left(\frac{1+h x}{h}\right)^{\nu} e^{\frac{h \Theta}{1+h x}} F$

Let $h$ become infinite; then $\frac{h}{1+h x}, \frac{h y}{1+h x}$ and $(-)^{w}\left(\frac{1+h x}{h}\right)^{\nu} e^{\frac{h \Theta}{1+h x}} F^{\prime}$ become $\frac{1}{x}, \frac{y}{x}$ and $(-)^{w} x^{\nu} e^{\frac{\Theta}{x}} F$, showing that the substitution $\frac{1}{x}, \frac{y}{x}$ changes $F$ into $(-)^{w} x^{\nu} e^{\frac{\Theta}{x}} F$. 


\section{LECTURE XXV.}

In a letter to me dated June 14th, 1886, M. Halphen calls forms which are persistent under the substitution $\frac{1}{x}, \frac{y}{x}$, Invariants d'homologie. He uses the letters

$$
a_{0}, a_{1}, a_{2}, a_{3}, \ldots a_{n}
$$

to denote $y$ and its successive modified derivatives with respect to $x$; and, supposing them to become

$$
A_{0}, A_{1}, A_{2}, A_{3}, \ldots A_{n},
$$

in consequence of the substitution $\frac{1}{x}, \frac{y}{x}$, gives, in the briefest possible manner, two very ingenious proofs of the formula

$$
A_{n}=(-)^{n} x^{2 n-1}\left\{a_{n}+\frac{n-2}{1 . x} a_{n-1}+\frac{(n-2)(n-3)}{1.2 . x^{2}} a_{n-2}+\ldots\right\},
$$

from which he deduces the theorem that the substitution in question changes any homogeneous and isobaric function $f$, of degree $i$ and weight $\omega$ in

where $\Theta$ is the partial differential operator

$$
\begin{gathered}
a_{0}, a_{1}, a_{2}, a_{3}, \ldots a_{n}, \\
F=(-)^{\omega} x^{2 \omega-i} e^{\frac{\Theta}{x}} f,
\end{gathered}
$$

$$
-a_{0} \partial_{a_{1}}+a_{2} \partial_{a_{3}}+2 a_{3} \partial_{a_{4}}+\ldots+(n-2) a_{n-1} \partial_{a_{n}}
$$

I give the two proofs mentioned above in M. Halphen's own words, adding occasional footnotes, and making slight changes in the literation of his formulae when it seems desirable to do so.

Soient

$$
X=\frac{1}{x}, Y=\frac{y}{x} \text {. }
$$

Par une formule connue (Schlömilch, Compendium II.)

$$
\frac{d^{n} y}{d X^{n}}=(-1)^{n} x^{n+1} \frac{d^{n}}{d x^{n}}\left(x^{n-1} y\right)^{*}
$$

* An easy inductive proof of this may be obtained as follows :

Since

$$
\frac{d}{d X}=-x^{2} \frac{d}{d x} \text { we have } \frac{d^{\kappa+1} y}{d X^{\kappa+1}}=-x^{2} \frac{d}{d x}\left(\frac{d^{\kappa} y}{d X^{\kappa}}\right) .
$$

Hence, assuming the truth of the formula when $n=\kappa$, we find

$$
\begin{aligned}
\frac{d^{\kappa+1} y}{d X^{\kappa+1}} & =(-)^{\kappa+1} x^{2} \frac{d}{d x}\left\{x^{\kappa+1} \frac{d^{\kappa}}{d x^{\kappa}}\left(x^{\kappa-1} y\right)\right\} \\
& =(-)^{\kappa+1} x^{2}\left\{x^{\kappa+1} \frac{d^{\kappa+1}}{d x^{\kappa+1}}\left(x^{\kappa-1} y\right)+(\kappa+1) x^{\kappa} \frac{d^{\kappa}}{d x^{\kappa}}\left(x^{\kappa-1} y\right)\right\} \\
& =(-)^{\kappa+1} x^{\kappa+2}\left\{x \frac{d^{\kappa+1}}{d x^{\kappa+1}}\left(x^{\kappa-1} y\right)+(\kappa+1) \frac{d^{\kappa}}{d x^{\kappa}}\left(x^{\kappa-1} y\right)\right\} \\
& =(-)^{\kappa+1} x^{\kappa+2} \frac{d^{\kappa+1}}{d x^{\kappa+1}}\left(x^{\kappa} y\right) .
\end{aligned}
$$

Thus, if the formula is true for $n=\kappa$, it will be equally so when $n=\kappa+1$. But it is obviously true when $n=1$ (when it becomes $\frac{d y}{d X}=-x^{2} \frac{d y}{d x}$ ), and therefore holds universally. 
et puisque

$$
Y=X y
$$

il en resulte

$$
\begin{aligned}
\frac{d^{n} Y}{d X^{n}} & =X \frac{d^{n} y}{d X^{n}}+n \frac{d^{n-1} y}{d X^{n-1}}=(-1)^{n} x^{n}\left\{\frac{d^{n}}{d x^{n}}\left(x^{n-1} y\right)-n \frac{d^{n-1}}{d x^{n-1}}\left(x^{n-2} y\right)\right\} \\
& =(-1)^{n} x^{2 n-1}\left\{y_{n}+\frac{n(n-2)}{1 \cdot x} y_{n-1}+\frac{n(n-1)(n-2)(n-3)}{1.2 . x^{2}} y_{n-2}+\ldots\right\} *
\end{aligned}
$$

Si l'on pose

$$
\frac{d^{n} Y}{d X^{n}}=n ! A_{n}, y_{n}=n ! a_{n}
$$

il vient

$$
A_{n}=(-1)^{n} x^{2 n-1}\left\{a_{n}+\frac{n-2}{1 \cdot x} a_{n-1}+\frac{(n-2)(n-3)}{1 \cdot 2 \cdot x^{2}} a_{n-2}+\ldots\right\}
$$

Soit

$$
\Theta f=\Sigma(n-2) a_{n-1} \frac{\partial f}{\partial a_{n}} \dagger
$$

on aura

$$
\begin{aligned}
& \Theta a_{n}=(n-2) a_{n-1}, \\
& \Theta^{2} a_{n}=(n-2)(n-3) a_{n-2}, \\
& A_{n}=(-1)^{n} x^{2 n-1}\left\{a_{n}+\frac{1}{1 \cdot x} \Theta a_{n}+\frac{1}{1.2 \cdot x^{2}} \Theta^{2} a_{n}+\ldots\right\} .
\end{aligned}
$$

Par conséquent, pour une fonction contenant $a_{0}, a_{1}, a_{2}, \ldots$, de degré $i$ et de poids $\omega$, à chaque terme, on aura

$$
F=(-1)^{\omega} x^{2 \omega-i}\left\{f+\frac{1}{1 \cdot x} \Theta f+\frac{1}{1.2 \cdot x^{2}} \Theta^{2} f+\ldots\right\}+. \quad \text { C.Q.F.D. }
$$

* For, expanding by Leibnitz's Theorem,

$$
\begin{aligned}
\frac{d^{n}}{d x^{n}}\left(x^{n-1} y\right)-n \frac{d^{n-1}}{d x^{n-1}}\left(x^{n-2} y\right)= & x^{n-1} y_{n}+n(n-1) x^{n-2} y_{n-1}+\frac{n(n-1)}{1.2}(n-1)(n-2) x^{n-3} y_{n-2}+\ldots \\
& -n\left\{x^{n-2} y_{n-1}+(n-1)(n-2) x^{n-3} y_{n-2}+\ldots\right\} \\
= & x^{n-1} y_{n}+n(n-2) x^{n-2} y_{n-1}+\frac{n(n-1)(n-2)(n-3)}{1.2} x^{n-3} y_{n-2}+\ldots .
\end{aligned}
$$

+ The summation extending to all positive integral values of $n$, from 1 to $\infty$, so that

$$
\Theta=-a_{0} \partial_{a_{1}}+a_{2} \partial_{a_{3}}+2 a_{3} \partial_{a_{4}}+3 a_{4} \partial_{a_{5}}+\ldots .
$$

Remembering that Halphen's $a_{0}, a_{1}, a_{2}, a_{3}, \ldots$ have the same meaning as our $y, t, a, b, \ldots$, this operator is $-y \partial_{t}+a \partial_{b}+2 b \partial_{c}+3 c \partial_{d}+\ldots$ identical with the $\theta$ used in previous lectures.

$\ddagger$ We may show without much difficulty that, when $\theta_{1}, \theta_{2}, \theta_{3}, \ldots$ are each of them equivalent to $\theta$, but $\theta_{1}$ acts on $u$ only, $\theta_{2}$ on $v, \Theta_{3}$ on $w$, and so on, $\theta_{u v w} \ldots=\left(\theta_{1}+\theta_{2}+\theta_{3}+\ldots\right) u v w \ldots$. From this it can be deduced that $\theta^{\kappa} u v w \ldots=\left(\theta_{1}+\theta_{2}+\theta_{3}+\ldots\right)^{\kappa} u v w \ldots$, when $\kappa$ is any positive integer. Now let the number of the functions $u, v, w, \ldots$ be $i$, and suppose that

$$
u=a_{n}, v=a_{p}, w=a_{q}, \ldots ;
$$

suppose, also, that the weight $n+p+q+\ldots=\omega$. Then

$$
\begin{aligned}
A_{n} A_{p} A_{q} \ldots & =(-)^{\omega} x^{2 \omega-i}\left(e^{\frac{\Theta}{x}} a_{n}\right)\left(\frac{\Theta}{e^{x}} a_{p}\right)\left(\frac{\Theta}{e} a_{q}\right) \ldots=(-)^{\omega} x^{2 \omega-i} e^{\frac{1}{\left(\Theta_{1}+\Theta_{2}+\Theta_{3}+\ldots\right)} a_{n} a_{p} a_{q} \ldots} \\
& =(-)^{\omega} x^{2 \omega-i} e^{\frac{\Theta}{x}} a_{n} a_{p} a_{q} \ldots
\end{aligned}
$$

(for by what precedes $\Theta_{1}+\Theta_{2}+\Theta_{3}+\ldots$ may be replaced by $\Theta$ ). Taking $a_{n} a_{p} a_{q} \ldots$ and $A_{n} A_{p} A_{q} \ldots$ to be corresponding terms of $f$ and $F$, we see at once that

$$
F=(-)^{\omega} x^{2 \omega-i} e_{e}^{\stackrel{\Theta}{x}} f .
$$


Autre Demonstration de la Formule (I)*.

Si l'on change $X$ et $x$ en $X+H$ et $x+h$, on a

$$
h=-\frac{H}{X(X+H)} \text {. }
$$

Maintenant la formule

écrite symboliquement +

$$
y=a_{0}+h a_{1}+h^{2} a_{2}+\ldots+h^{n} a_{n}+\ldots
$$

$$
y=\frac{1}{1-a h}
$$

devient

$$
y=\frac{X(X+H)}{X^{2}+H(X+a)}
$$

D'ailleurs

$$
Y=(X+H) y \text {; }
$$

done symboliquement

$$
Y=\frac{X(X+H)^{2}}{X^{2}+H(X+a)} .
$$

Si l'on développe le second membre (II) suivant les puissances ascendants de $H$, le coefficient de $H^{n}$ est $A_{n}$. Or ce développement est

$$
\begin{aligned}
Y=X\left\{1+\left(1-\frac{a}{X}\right) \frac{H}{X}\right. & +\left(\frac{a}{X}\right)^{2}\left(\frac{H}{X}\right)^{2} \\
& \left.+\ldots+(-1)^{n}\left(\frac{H}{X}\right)^{n}\left(1+\frac{a}{X}\right)^{n-2}\left(\frac{a}{X}\right)^{2}+\ldots\right\}
\end{aligned}
$$

* If $x$ becomes $x+h$ in consequence of the augmentation of $X$ by an arbitrary quantity $H$, the increment of $x$ will not be a constant, but will depend on $X$ as well as on $H$. The value of $h$ may be found at once by eliminating $x$ between $X=\frac{1}{x}$ and $X+H=\frac{1}{x+h}$, when we obtain $X+H=\frac{X}{1+h X}$, and consequently $h=-\frac{H}{X(X+H)}$.

This increase of $X$ also changes $y$ and $Y$ (functions of $x$ and $X$, whose original values were $a_{0}$ and $A_{0}$ before the augmentation of $X$ took place) into

$$
\begin{aligned}
y & =a_{0}+h a_{1}+h^{2} a_{2}+\ldots+h^{n} a_{n}+\ldots \\
\text { and into } & Y=A_{0}+H A_{1}+H^{2} A_{2}+\ldots+H^{n} A_{n}+\ldots .
\end{aligned}
$$

These altered values of $y$ and $Y$ are the ones used in this second proof; the other letters retain their original signification.

+ The word symboliquement indicates, whenever it is used, that powers of $a$ are to be replaced by suffixes of corresponding value. For example, in the final result

is to be replaced by

$$
A_{n}=(-)^{n} x^{2 n-1}\left(a^{n}+\frac{n-2}{x} a^{n-1}+\ldots\right)
$$

$$
A_{n}=(-)^{n} x^{2 n-1}\left(a_{n}+\frac{n-2}{x} a_{n-1}+\ldots\right) \text {. }
$$

In our notation the final result is $A_{n+2}=(-)^{n} x^{2 n+3}\left(a, b, c, d, \ldots \ell \frac{1}{x}, 1\right)^{n}$. 
donc symboliquement

$$
A_{n}=(-1)^{n} \frac{1}{X^{n+1}}\left(1+\frac{a}{X}\right)^{n-2} a^{2}=(-1)^{n} x^{2 n-1}\left(a+\frac{1}{x}\right)^{n-2} a^{2}
$$

ce qui est justement la formule (I).

We may regard the coefficients $a, b, c, \ldots$ of the ordinary binary Quantic in $u, v$,

$$
(a, b, c, \ldots \gamma u, v)^{n} \text {, }
$$

as the successive modified derivatives, beginning with the second, of a new variable $y$ with respect to another new variable $x$.

Any invariant $I$ of this Quantic will then retain its form unaltered, or at most merely acquire an extraneous factor, if

(1) leaving $x, y, v$ unaltered we change $u$ into $u+\lambda v$,

$$
\begin{aligned}
& \text { " } u, v \quad " \quad \quad \quad, \quad x, y \quad " \quad \frac{x}{1+h x}, \frac{y}{1+h x}, \\
& u, v \quad " \quad \quad \quad, \quad x, y \quad, \quad \frac{1}{x}, \quad \frac{y}{x},
\end{aligned}
$$

where $\lambda$ and $h$ are arbitrary constants.

For we have seen that these three substitutions will severally convert any homogeneous and isobaric function $F$, of degree $i$ and weight $w$ in the letters $a, b, c, \ldots$, into

$$
e^{\lambda \Omega} F,(1+h x)^{\nu} e^{\frac{h \Omega}{1+h x}} F, \text { and }(-)^{w} x^{\nu} e^{\frac{\Omega}{x}} F,
$$

where, in each case, $\Omega=a \partial_{b}+2 b \partial_{c}+3 c \partial_{d}+\ldots$, and $\nu=3 i+2 w$. From our point of view an invariant is defined as a homogeneous and isobaric solution of the equation

$$
\Omega I=0 .
$$

Hence the above substitutions convert the invariant $I$ into

$$
I,(1+h x)^{\nu} I \text {, and }(-)^{w} x^{\nu} I \text {, respectively. }
$$

An absolute invariant with respect to any substitution is one which, disregarding its sign, remains unchanged in absolute value by that substitution. Thus, any invariant for which

$$
\nu=3 i+2 w=0
$$

is an absolute invariant with respect to each of the three substitutions here considered.

An invariant is of odd or even character with respect to any substitution according as its sign is or is not changed by that substitution. Thus, invariants are of odd or even character with respect to the substitution $\frac{1}{x}, \frac{y}{x}$ according as their weights are odd or even. 
This corresponds to the theorem that the character (with respect to the interchange of $x$ and $y$ ) of a pure reciprocant is odd or even according as its degree is odd or even [p. 316, above].

From any two invariants for which $\nu$ has the same value we can form an absolute invariant (that is, one for which $\nu=0$ ) by taking their ratio, and then by differentiating the absolute invariant thus formed obtain another invariant.

Suppose $\quad I_{1}$ to be an invariant of degree $i_{1}$ and weight $w_{1}$,

and let

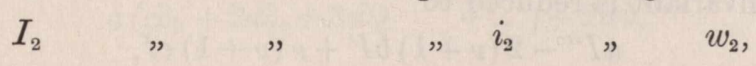

$$
" 3 i_{1}+2 w_{1}=\nu_{1}, 3 i_{2}+2 w_{2}=\nu_{2}
$$

then the $\nu$ for $I_{1}{ }^{\nu_{2}}$ is the same as that for $I_{2}^{\nu_{1}}$, and consequently $I_{1}^{\nu_{2}} I_{2}{ }^{-\nu_{1}}$ is an absolute invariant.

We proceed to show that $\frac{d}{d x}\left(I_{1}^{\nu_{2}} I_{2}-\nu_{1}\right)$ is an invariant, though not an absolute one.

Using accents to denote differential derivation with respect to $x$, we have

$$
\frac{d}{d x}\left(I_{1}^{\nu_{2}} I_{2}^{-\nu_{1}}\right)=I_{1}^{\nu_{2}-1} I_{2}^{-\nu_{1}-1}\left(\nu_{2} I_{1}^{\prime} I_{2}-\nu_{1} I_{1} I_{2}^{\prime}\right)
$$

If, then, we can prove that $\nu_{2} I_{1}^{\prime} I_{2}-\nu_{1} I_{1} I_{2}^{\prime}$ is an invariant, it will follow that $\frac{d}{d x}\left(I_{1}^{\nu_{2}} I_{2}{ }^{-\nu_{1}}\right)$ will be one also, and the proposition will be established. It may be very easily shown that this is the case by using Cayley's generators $P$ and $Q$. For [p. 327, above], $I$ being any invariant of degree $i$ and weight $w, P I$ and $Q I$ are also invariants where

and

$$
P=a\left(b \partial_{a}+c \partial_{b}+d \partial_{c}+e \partial_{d}+\ldots\right)-i b
$$

$$
Q=a\left(c \partial_{b}+2 d \partial_{c}+3 e \partial_{d}+\ldots\right)-2 w b \text {. }
$$

Hence

$$
(3 P+Q) I \text { is an invariant. }
$$

Now, since

and

$$
3 b \partial_{a}+4 c \partial_{b}+5 d \partial_{c}+\ldots=\frac{d}{d x}
$$

$$
(3 P+Q) I=a\left(3 b \partial_{a}+4 c \partial_{b}+5 d \partial_{c}+\ldots\right) I-(3 i+2 w) b I=a I^{\prime}-\nu b I .
$$

Consequently $\quad a I_{1}^{\prime}-\nu_{1} b I_{1}$ and $a I_{2}^{\prime}-\nu_{2} b I_{2}$

are both of them invariants. Hence the combination

$$
\nu_{2} I_{2}\left(a I_{1}^{\prime}-\nu_{1} b I_{1}\right)-\nu_{1} I_{1}\left(a I_{2}^{\prime}-\nu_{2} b I_{2}\right)=a\left(\nu_{2} I_{1}^{\prime} I_{2}-\nu_{1} I_{1} I_{2}^{\prime}\right)
$$

is also an invariant; that is

$$
\nu_{2} I_{1}^{\prime} I_{2}-\nu_{1} I_{1} I_{2}^{\prime}
$$

is one; which is the theorem to be demonstrated.

s. Iv. 
The invariant $a I^{\prime}-\nu b I$, which we generated from $I$, is of degree $i+1$ and weight $w+1$; its $\nu$ is therefore the original $\nu$ increased by 5 units, three for the unit increase in the degree and two for the unit increase in the weight. Hence, on repeating the process of generation, we obtain the invariant

$$
\left\{a \frac{d}{d x}-(\nu+5) b\right\}\left(a I^{\prime}-\nu b I\right)=a^{2} I^{\prime \prime}-2(\nu+1) a b I^{\prime}-4 \nu a c I+\nu(\nu+5) b^{2} I .
$$

By adding on the invariant $\nu(\nu+5)\left(a c-b^{2}\right) I$ and dividing the sum by $a$, the above invariant is reduced to

$$
a I^{\prime \prime}-2(\nu+1) b I^{\prime}+\nu(\nu+1) c I
$$

which is an invariant of lower degree by unity than the unreduced form.

The results obtained above may be compared with the corresponding ones in the theory of reciprocants.

Thus to the invariants

$$
I \text { (deg. } i \text {, wt. } w)
$$

$$
a I^{\prime}-\nu b I \text {, }
$$$$
\nu_{2} I_{1}^{\prime} I_{2}-\nu_{1} I_{1} I_{2}^{\prime} \text {, }
$$

$a I^{\prime \prime}-2(\nu+1) b I^{\prime}+\nu(\nu+1) c I$,

where $\quad \nu=3 i+2 w$, correspond the reciprocants

$R$ (deg. $i$, wt. $w$ ), $a R^{\prime}-\mu b R$, $\mu_{2} R_{1}^{\prime} R_{2}-\mu_{1} R_{1} R_{2}^{\prime}$,

$5 a R^{\prime \prime}-5(2 \mu+1) b R^{\prime}+4 \mu(\mu-1) c R$, where $\quad \mu=3 i+w$.

Defining a plenarily absolute form to be one whose degree and weight are both zero $(i=0, w=0)$, the theorem I shall now prove may be stated as follows :

By differentiating a plenarily absolute principiant we obtain another principiant.

Let $P$ be any principiant of degree $i$ and weight $w$. Then, by what precedes, since $P$ is both an invariant and a reciprocant,

and

$$
\begin{aligned}
& a \frac{d P}{d x}-\nu b P \text { is an invariant, } \\
& a \frac{d P}{d x}-\mu b P \text { is a reciprocant. }
\end{aligned}
$$

Hence, when $\nu=0$ (that is, when $3 i+2 w=0$ ),

$$
\frac{d P}{d x} \text { is an invariant, }
$$

and when $\mu=0$ (that is, when $3 i+w=0$ ),

$$
\frac{d P}{d x} \text { is a reciprocant. }
$$

When both $\mu=0$ and $\nu=0$ (which happens when $i=0, w=0$ ),

$$
\frac{d P}{d x} \text { is both a reciprocant and an invariant; }
$$

that is,

$$
\frac{d P}{d x} \text { is a principiant. }
$$




\section{LECTURE XXVI.}

In the theory of Invariants the annihilator $\Omega$ has two independent reversors any linear combination of which will also be a reversor. To each of these reversors there corresponds a generator for invariants. Thus Cayley's two generators

$$
\begin{aligned}
& a\left(b \partial_{a}+c \partial_{b}+d \partial_{c}+e \partial_{d}+\ldots\right)-i b \\
& a\left(c \partial_{b}+2 d \partial_{c}+3 e \partial_{d}+\ldots\right)-2 w b
\end{aligned}
$$

correspond to the two reversors

$$
\begin{aligned}
& b \partial_{a}+c \partial_{b}+d \partial_{c}+e \partial_{d}+\ldots \\
& c \partial_{b}+2 d \partial_{c}+3 e \partial_{d}+\ldots
\end{aligned}
$$

The only linear combination of these which does not increase the extent $j$ as well as the weight of the operand is

$$
0=j b \partial_{a}+(j-1) c \partial_{b}+(j-2) d \partial_{c}+\ldots
$$

It is convenient to take this for one of our reversors, and for the other

$$
\frac{d}{d x}=3 b \partial_{a}+4 c \partial_{b}+5 d \partial_{c}+\ldots
$$

which is a reversor to $V$, the annihilator for reciprocants, as well as to $\Omega$, the annihilator for invariants.

We saw in Lecture XI. [p. 364, above] that when $F$ is any homogeneous and isobaric function of degree $i$ and weight $w$ in the $j+1$ letters $a, b, c, \ldots$

$$
(\Omega O-O \Omega) F=(i j-2 w) F .
$$

The method employed in proving this can also be applied to show that

$$
\left(\Omega \frac{d}{d x}-\frac{d}{d x} \Omega\right) F=\nu F
$$

where $\nu=3 i+2 w$.

Corresponding to the reversors $O$ and $\frac{d}{d x}$ we have the two generators for invariants

$$
a \frac{d}{d x}-\nu b \text { and } a O-(\ddot{j}-2 w) b
$$

which are linear combinations of Cayley's generators.

Thus, if $I$ be any invariant,

$$
\left(a \frac{d}{d x}-\nu b\right) I \text { and }\{a O-(i j-2 w) b\} I
$$

are also invariants. 
The operator $\frac{d}{d x}$ has, but $O$ has not, analogous properties in the theory of Reciprocants; namely, $\frac{d}{d x}$ is a reversor to $V$ and $a \frac{d}{d x}-\mu b$ is a generator for reciprocants. Thus, we have shown in previous lectures that

$$
\left(V \frac{d}{d x}-\frac{d}{d x} V\right) F=2 \mu a F
$$

where $F$ is any homogeneous and isobaric function, and $\mu=3 i+w$, and that if $R$ is any pure reciprocant $\left(a \frac{d}{d x}-\mu b\right) R$ is one also.

Now, Mr Hammond has found that if

$$
W=\frac{b}{a} \partial_{a}+\frac{2 a c-b^{2}}{a^{2}} \partial_{b}+\frac{3 a^{2} d-3 a b c+b^{3}}{a^{3}} \partial_{c}+\ldots
$$

$W$ is a reversor to $V$, and $a^{2} W-i b$ is a generator for pure reciprocants. In fact we have

$$
\begin{aligned}
V W-W V & =V\left(\frac{b}{a}\right) \partial_{a} \\
& +\left\{V\left(\frac{2 a c-b^{2}}{a^{2}}\right)-W\left(2 a^{2}\right)\right\} \partial_{b} \\
& +\left\{V\left(\frac{3 a^{2} d-3 a b c+b^{3}}{a^{3}}\right)-W(5 a b)\right\} \partial_{c} \\
& +\ldots \ldots \ldots \ldots \ldots \ldots \ldots \ldots \ldots \ldots \ldots \ldots \ldots \ldots \ldots \ldots \ldots \ldots \ldots \ldots \ldots \ldots
\end{aligned}
$$

But, since

$$
\begin{array}{ll}
V\left(\frac{b}{a}\right) & =2 a \\
V\left(\frac{2 a c-b^{2}}{a^{2}}\right) & =10 b-4 b=6 b \\
V\left(\frac{3 a^{2} d-3 a b c+b^{3}}{a^{3}}\right) & =\left(18 c+9 \frac{b^{2}}{a}\right)-\left(15 \frac{b^{2}}{a}+6 c\right)+6 \frac{b^{2}}{a}=12 c
\end{array}
$$

and

$$
\begin{aligned}
& W\left(2 a^{2}\right)=4 b \\
& W(5 a b)=5 \frac{b^{2}}{a}+5\left(\frac{2 a c-b^{2}}{a}\right)=10 c,
\end{aligned}
$$

it follows that

$$
V W-W V=2 a \partial_{a}+2 b \partial_{b}+2 c \partial_{c}+\ldots=2 i .
$$

Thus $W$ is a reversor to $V$. Moreover, $a^{2} W-i b$ acting on any pure reciprocant generates another.

Let $R$ be a pure reciprocant of degree $i$; then, by what precedes,

$$
(V W-W V) R=2 i R \text {. }
$$


But, since $R$ is a pure reciprocant, $V R=0$, and consequently $V W R=2 i R$.

Now, $\quad V\left(a^{2} W-i b\right) R=a^{2} V W R-i R V b=a^{2} .2 i R-i R .2 a^{2}=0$.

Hence $\quad\left(a^{2} W-i b\right) R$

is a pure reciprocant; that is $a^{2} W-i b$

is a generator for pure reciprocants.

$\mathrm{Mr}$ Hammond shows that $W$ is a reversor to $V$ in the following manner :

Let

$$
\begin{aligned}
u & =a_{0}+a_{1} e^{\theta}+a_{2} e^{2 \theta}+a_{3} e^{3 \theta}+\ldots, \\
\phi(u) & =A_{0}+A_{1} e^{\theta}+A_{2} e^{2 \theta}+A_{3} e^{3 \theta}+\ldots, \\
\psi(u) & =A_{0}^{\prime}+A_{1}^{\prime} e^{\theta}+A_{2}^{\prime} e^{2 \theta}+A_{3}^{\prime} e^{3 \theta}+\ldots,
\end{aligned}
$$

and consider the operators

$$
\begin{aligned}
& P=\lambda A_{0} \partial_{a_{n}}+(\lambda+\mu) A_{1} \partial_{a_{n+1}}+(\lambda+2 \mu) A_{2} \partial_{a_{n+2}}+\ldots \\
& Q=\lambda^{\prime} A_{0}^{\prime} \partial_{a_{n^{\prime}}}+\left(\lambda^{\prime}+\mu^{\prime}\right) A_{1} \partial_{a_{n^{\prime}+1}}+\left(\lambda^{\prime}+2 \mu^{\prime}\right) A_{2}^{\prime} \partial_{a_{n^{\prime}+2}}+\ldots
\end{aligned}
$$

Regarding $e^{\theta}$ as an operative symbol defined by the equation

we may write

$$
e^{\kappa \theta}\left[\partial_{a_{0}}\right]=\partial_{a_{\kappa}},
$$

$$
\begin{aligned}
P= & \left\{\lambda A_{0} e^{n \theta}+(\lambda+\mu) A_{1} e^{(n+1) \theta}+(\lambda+2 \mu) A_{2} e^{(n+2) \theta}+\ldots\right\}\left[\partial_{a_{0}}\right] \\
= & e^{n \theta} \lambda\left(A_{0}+A_{1} e^{\theta}+A_{2} e^{2 \theta}+\ldots\right)\left[\partial_{a_{0}}\right] \\
& \quad+e^{n \theta} \mu\left(A_{1} e^{\theta}+2 A_{2} e^{2 \theta}+\ldots\right)\left[\partial_{a_{0}}\right] \\
= & e^{n \theta}\left(\lambda+\mu \frac{d}{d \theta}\right) \phi(u)\left[\partial_{a_{0}}\right] .
\end{aligned}
$$

Similarly,

$$
Q=e^{n^{\prime} \theta}\left(\lambda^{\prime}+\mu^{\prime} \frac{d}{d \theta}\right) \psi(u)\left[\partial_{a_{0}}\right]
$$

$$
\text { Now, } \begin{aligned}
P Q-Q P & =\left\{P e^{n^{\prime} \theta}\left(\lambda^{\prime}+\mu^{\prime} \frac{d}{d \theta}\right) \psi(u)-Q e^{n \theta}\left(\lambda+\mu \frac{d}{d \theta}\right) \phi(u)\right\}\left[\partial_{a_{0}}\right] \\
& =\left\{e^{n^{\prime} \theta}\left(\lambda^{\prime}+\mu^{\prime} \frac{d}{d \theta}\right) P \psi(u)-e^{n \theta}\left(\lambda+\mu \frac{d}{d \theta}\right) Q \phi(u)\right\}\left[\partial_{a_{0}}\right] .
\end{aligned}
$$

For

$$
Q \phi(u)=Q A_{0}+e^{\theta} Q A_{1}+e^{2 \theta} Q A_{2}+\ldots ;
$$

so that

$$
e^{n \theta} \frac{d}{d \theta} Q \phi(u)=e^{n \theta}\left(e^{\theta} Q A_{1}+2 e^{2 \theta} Q A_{2}+\ldots\right)
$$

and

$$
e^{n \theta} \frac{d}{d \theta} \phi(u)=e^{n \theta}\left(e^{\theta} A_{1}+2 e^{2 \theta} A_{2}+\ldots\right) ;
$$

so that

$$
Q e^{n \theta} \frac{d}{d \theta} \phi(u)=e^{n \theta}\left(e^{\theta} Q A_{1}+2 e^{2 \theta} Q A_{2}+\ldots\right)
$$

$$
=e^{n \theta} \frac{d}{d \theta} Q \phi(u) \text {. }
$$

Similarly,

$$
P e^{n^{\prime} \theta} \frac{d}{d \theta} \psi(u)=e^{n^{\prime} \theta} \frac{d}{d \theta} P \psi(u)
$$


Moreover,

$$
\begin{aligned}
P \psi(u) & =\psi^{\prime}(u) P u=\psi^{\prime}(u) P\left(a_{0}+a_{1} e^{\theta}+a_{2} e^{2 \theta}+\ldots\right) \\
& =\psi^{\prime}(u)\left\{e^{n \theta} \lambda A_{0}+e^{(n+1) \theta}(\lambda+\mu) A_{1}+e^{(n+2) \theta}(\lambda+2 \mu) A_{2}+\ldots\right\} \\
& =e^{n \theta} \psi^{\prime}(u)\left(\lambda+\mu \frac{d}{d \theta}\right) \phi(u) .
\end{aligned}
$$

Similarly, $\quad Q \phi(u)=e^{n^{\prime} \theta} \phi^{\prime}(u)\left(\lambda^{\prime}+\mu^{\prime} \frac{d}{d \theta}\right) \psi(u)$.

Hence

$$
\begin{aligned}
P Q-Q P= & \left\{e^{n^{\prime} \theta}\left(\lambda^{\prime}+\mu^{\prime} \frac{d}{d \theta}\right) e^{n \theta} \psi^{\prime}(u)\left(\lambda+\mu \frac{d}{d \theta}\right) \phi(u)\right. \\
& \left.-e^{n \theta}\left(\lambda+\mu \frac{d}{d \theta}\right) e^{n^{\prime} \theta} \phi^{\prime}(u)\left(\lambda^{\prime}+\mu^{\prime} \frac{d}{d \theta}\right) \psi(u)\right\}\left[\partial_{a_{0}}\right] \\
= & e^{\left(n+n^{\prime}\right) \theta}\left\{\left(\lambda^{\prime}+\mu^{\prime} n+\mu^{\prime} \frac{d}{d \theta}\right) \psi^{\prime}(u)\left(\lambda+\mu \frac{d}{d \theta}\right) \phi(u)\right. \\
& \left.-\left(\lambda+\mu n^{\prime}+\mu \frac{d}{d \theta}\right) \phi^{\prime}(u)\left(\lambda^{\prime}+\mu^{\prime} \frac{d}{d \theta}\right) \psi(u)\right\}\left[\partial_{a_{0}}\right] .
\end{aligned}
$$

If in this we write

$$
\begin{array}{ll}
\phi=\frac{u^{2}}{2}, & \lambda=4, \quad \mu=1, \quad n=1, \\
\psi=\log u, & \lambda^{\prime}=0, \mu^{\prime}=1, \quad n^{\prime}=-1,
\end{array}
$$

we have

$$
\begin{aligned}
P Q-Q P & =\left\{\left(1+\frac{d}{d \theta}\right) u^{-1}\left(4+\frac{d}{d \theta}\right) \frac{u^{2}}{2}-\left(3+\frac{d}{d \theta}\right) u \frac{d}{d \theta} \log u\right\}\left[\partial_{a_{0}}\right] \\
& =\left\{\left(1+\frac{d}{d \theta}\right)\left(2 u+\frac{d u}{d \theta}\right)-\left(3+\frac{d}{d \theta}\right) \frac{d u}{d \theta}\right\}\left[\partial_{a_{0}}\right] \\
& =\left\{\left(1+\frac{d}{d \theta}\right)\left(2+\frac{d}{d \theta}\right)-\left(3+\frac{d}{d \theta}\right) \frac{d}{d \theta}\right\} u\left[\partial_{a_{0}}\right] \\
& =2 u\left[\partial_{a_{0}}\right]
\end{aligned}
$$

Now,

$$
2 u\left[\partial_{a_{0}}\right]=2\left(a_{0}+a_{1} e^{\theta}+a_{2} e^{2 \theta}+\ldots\right)\left[\partial_{a_{0}}\right]
$$

Also

$$
\begin{gathered}
=2\left(a_{0} \partial_{a_{0}}+a_{1} \partial_{a_{1}}+a_{2} \partial_{a_{2}}+\ldots\right) \\
P=4 A_{0} \partial_{a_{1}}+5 A_{1} \partial_{a_{2}}+6 A_{2} \partial_{a_{3}}+\ldots \\
Q=A_{1}^{\prime} \partial_{a_{0}}+2 A_{2}^{\prime} \partial_{a_{1}}+3 A_{3}^{\prime} \partial_{a_{2}}+\ldots
\end{gathered}
$$

where

$$
\frac{1}{2}\left(a_{0}+a_{1} e^{\theta}+a_{2} e^{2 \theta}+\ldots\right)^{2}=A_{0}+A_{1} e^{\theta}+A_{2} e^{2 \theta}+\ldots
$$

and

$$
\log \left(a_{0}+a_{1} e^{\theta}+a_{2} e^{2 \theta}+\ldots\right)=\log a_{0}+A_{1}^{\prime} e^{\theta}+A_{2}{ }^{\prime} e^{2 \theta}+\ldots .
$$

Equating coefficients, we have

$$
\begin{gathered}
A_{0}=\frac{1}{2} a_{0}^{2}, A_{1}=a_{0} a_{1}, A_{2}=a_{0} a_{2}+\frac{a_{1}^{2}}{2}, \ldots \\
A_{1}^{\prime}=\frac{a_{1}}{a_{0}}, \quad A_{2}^{\prime}=\frac{2 a_{0} a_{2}-a_{1}{ }^{2}}{2 a_{0}{ }^{2}}, \ldots
\end{gathered}
$$


It is easily seen by expanding the logarithm that the general value of $A_{n}{ }^{\prime}$ is $(-)^{n+1} \frac{S_{n}}{n}$ where $S_{n}$ denotes the sum of the $n$th powers of the roots of

$$
a_{0} x^{n}+a_{1} x^{n-1}+a_{2} x^{n-2}+\ldots+a_{n} .
$$

Thus we have shown that if

$$
P=2 a_{0}^{2} \partial_{a_{1}}+5 a_{0} a_{1} \partial_{a_{2}}+\left(6 a_{0} a_{2}+3 a_{1}^{2}\right) \partial_{a_{3}}
$$

and

$$
Q=\frac{a_{1}}{a_{0}} \partial_{a_{0}}+\frac{2 a_{0} a_{2}-a_{1}^{2}}{a_{0}{ }^{2}} \partial_{a_{1}}+\frac{3 a_{0}{ }^{2} a_{3}-3 a_{0} a_{1} a_{2}+a_{1}^{3}}{a_{0}{ }^{3}} \partial_{a_{2}}+\ldots
$$

then

$$
P Q-Q P=2\left(a_{0} \partial_{a_{0}}+a_{1} \partial_{a_{1}}+a_{2} \partial_{a_{2}}+\ldots\right)=2 i .
$$

The general formula obtained for $P Q-Q P$ is an extension of a result of Capt. MacMahon's, who considers the case in which

$$
\phi(u)=\frac{u^{m}}{m}, \psi(u)=\frac{u^{m^{\prime}}}{m^{\prime}} .
$$

When $\phi(u)$ and $\psi(u)$ have these values, the general formula becomes $P Q-Q P=e^{\left(n+n^{\prime}\right) \theta}\left\{\left(\lambda^{\prime}+\mu^{\prime} n+\mu^{\prime} \frac{d}{d \theta}\right)\left(\frac{\lambda u^{m+m^{\prime}-1}}{m}+\mu u^{m+m^{\prime}-2} \frac{d u}{d \theta}\right)\right.$

But $\quad\left(\lambda^{\prime}+\mu^{\prime} n+\mu^{\prime} \frac{d}{d \theta}\right)\left(\frac{\lambda}{m} u^{m+m^{\prime}-1}+\mu u^{m+m^{\prime}-2} \frac{d u}{d \theta}\right)$

$$
=\left(\lambda^{\prime}+\mu^{\prime} n+\mu^{\prime} \frac{d}{d \theta}\right)\left(\frac{\lambda}{m}+\frac{\mu}{m+m^{\prime}-1} \frac{d}{d \theta}\right) u^{m+m^{\prime}-1} .
$$

Consequently

$$
P Q-Q P=e^{\left(n+n^{\prime}\right) \theta}\left\{\left(\lambda^{\prime}+\mu^{\prime} n+\mu^{\prime} \frac{d}{d \theta}\right)\left(\frac{\lambda}{m}+\frac{\mu}{m+m^{\prime}-1} \frac{d}{d \theta}\right)\right.
$$

In Capt. MacMahon's notation

in our notation

$$
P=(m, \lambda, \mu, n), Q=\left(m^{\prime}, \lambda^{\prime}, \mu^{\prime}, n^{\prime}\right) ;
$$

$$
\begin{aligned}
& P=e^{n \theta}\left(\lambda+\mu \frac{d}{d \theta}\right) \frac{u^{m}}{m}\left[\partial_{a_{0}}\right] \\
& Q=e^{n^{\prime} \theta}\left(\lambda^{\prime}+\mu^{\prime} \frac{d}{d \theta}\right) \frac{u^{m^{\prime}}}{m^{\prime}}\left[\partial_{a_{0}}\right]
\end{aligned}
$$

If now we write

$$
P Q-Q P=e^{\left(n+n^{\prime}\right) \theta}\left(\lambda_{1}+\mu_{1} \frac{d}{d \theta}\right) \frac{u^{m+m^{\prime}-1}}{m+m^{\prime}-1}\left[\partial_{a_{0}}\right]
$$

which is equivalent to

$$
P Q-Q P=\left(m+m^{\prime}-1, \lambda_{1}, \mu_{1}, n+n^{\prime}\right),
$$


we have $\quad\left(\lambda^{\prime}+\mu^{\prime} n+\mu^{\prime} \frac{d}{d \theta}\right)\left\{\frac{\lambda}{m}\left(m+m^{\prime}-1\right)+\mu \frac{d}{d \theta}\right\}$

$$
-\left(\lambda+\mu n^{\prime}+\mu \frac{d}{d \theta}\right)\left\{\frac{\lambda^{\prime}}{m^{\prime}}\left(m+m^{\prime}-1\right)+\mu^{\prime} \frac{d}{d \theta}\right\}=\lambda_{1}+\mu_{1} \frac{d}{d \theta} .
$$

Hence we obtain

$$
\begin{aligned}
& \lambda_{1}=\left(m+m^{\prime}-1\right)\left\{\frac{\lambda}{m}\left(\lambda^{\prime}+\mu^{\prime} n\right)-\frac{\lambda^{\prime}}{m^{\prime}}\left(\lambda+\mu n^{\prime}\right)\right\}, \\
& \mu_{1}=\mu \mu^{\prime}\left(n-n^{\prime}\right)+\frac{\lambda \mu^{\prime}}{m}\left(m^{\prime}-1\right)-\frac{\lambda^{\prime} \mu}{m^{\prime}}(m-1) .
\end{aligned}
$$

This agrees with Capt. MacMahon's result, a statement of which was given in Lecture XX. [above, p. 417].

Let $Q$ be a reversor to the operator $P=\lambda a^{m} \partial_{b}+(\ldots) \partial_{c}+(\ldots) \partial_{d}+\ldots$, and suppose that

$$
(P Q-Q P) F=\kappa a^{m-1} F,
$$

where $F$ is any homogeneous and isobaric function and $\kappa$ some number depending on its degree and weight. Then $\lambda a Q-\kappa b$ will be the generator corresponding to $Q$. In other words, we have to prove that

$$
P(\lambda a Q-\kappa b) F=0 \text { whenever } P F=0 .
$$

Now, by hypothesis, $P a=0, P b=\lambda a^{m}$, and when $P F=0$,

$$
P Q F=\kappa a^{m-1} F \text {. }
$$

Thus,

$$
\begin{aligned}
P(\lambda a Q-\kappa b) F & =\lambda a P Q F-\kappa F \cdot P b \\
& =\lambda \kappa a^{m} F-\lambda \kappa a^{m} F=0 .
\end{aligned}
$$

As an example, consider the case of the reversor $\frac{d}{d x}$ in the theory of reciprocants. Here

and since

$$
\begin{gathered}
P=V, \lambda=2, m=2 \\
\left(V \frac{d}{d x}-\frac{d}{d x} V\right) F=2 \mu a F
\end{gathered}
$$

we have $\kappa=2 \mu$. Hence the corresponding generator is $2\left(a \frac{d}{d x}-\mu b\right)$; or, disregarding the numerical factor 2 , we may take $a \frac{d}{d x}-\mu b$ for the generator in question, which is usually denoted by the letter $G$.

We may also write $G$ in the equivalent form

$$
G=4\left(a c-b^{2}\right) \partial_{b}+5(a d-b c) \partial_{c}+6(a e-b d) \partial_{d}+\ldots
$$

which it is sometimes more convenient to use.

I shall now show that

$$
\Omega G-G \Omega=a w-b \Omega,
$$

where $w$ is the weight of the operand. 
It is very easily seen that

$$
\begin{aligned}
& \Omega\left(a c-b^{2}\right)=0 \\
& \Omega(a d-b c)=2\left(a c-b^{2}\right) \\
& \Omega(a e-b d)=3(a d-b c) \\
& \Omega(a f-b e)=4(a e-b d)
\end{aligned}
$$

Hence it follows, by a direct and very simple calculation, that

$$
\Omega G-G \Omega=2\left(a c-b^{2}\right) \partial_{c}+3(a d-b c) \partial_{d}+4(a e-b d) \partial_{e}+\ldots .
$$

and

$$
b \partial_{b}+2 c \partial_{c}+3 d \partial_{d}+4 e \partial_{e}+\ldots=w,
$$

But, since

$$
a \partial_{b}+2 b \partial_{c}+3 c \partial_{d}+4 d \partial_{e}+\ldots=\Omega,
$$

$$
a w-b \Omega=2\left(a c-b^{2}\right) \partial_{c}+3(a d-b c) \partial_{d}+4(a e-b d) \partial_{e}+\ldots
$$

Consequently

$$
\Omega G-G \Omega=a w-b \Omega \text {. }
$$

The use of this formula will be seen in a subsequent lecture.

We may also prove an analogous theorem relating to the invariant generator $a \frac{d}{d x}-\nu b$, which we shall call $G^{\prime}$.

Let the operand be $F$, a homogeneous and isobaric function of degree $i$ and weight $w$. Then $V F$ is of degree $i+1$ and weight $w-1$; its $\nu$ is therefore

$$
3(i+1)+2(w-1)=\nu+1 .
$$

Thus, $\quad\left(V G^{\prime}-G^{\prime} V\right) F=\left\{V\left(a \frac{d}{d x}-\nu b\right)-\left(a \frac{d}{d x}-\nu b-b\right) V\right\} F^{\prime}$

$$
=a\left(V \frac{d}{d x}-\frac{d}{d x} V\right) F-\nu(V b-b V) F+b V F .
$$

But $\quad\left(V \frac{d}{d x}-\frac{d}{d x} V\right) F=2 \mu a F=2(3 i+w) a F$,

and

$$
V b F=b V F+2 a^{2} F .
$$

Consequently $V G^{\prime}-G^{\prime} V=2(3 i+w) a^{2} F-2 \nu a^{2} F+b V F$

$$
\begin{aligned}
& =2(3 i+w-\nu) a^{2} F+b V F^{\prime} \\
& =-2 w a^{2} F+b V F .
\end{aligned}
$$

It is perhaps worthy of notice that if $I$ is an invariant of weight $w$ and $R$ a pure reciprocant, also of weight $w$, then

whereas

$$
\Omega G I=a w I \text { and } V G^{\prime} R=-2 a^{2} w R ;
$$

$\Omega G^{\prime} I=0$ and $V G R=0$. 


\section{LECTURE XXVII.}

I should like to make a momentary pause in the development of the theory which now engages our attention and to revert to the proof of Cayley's theorem for the enumeration of linearly independent invariants contained in Lecture XI. and expressed by the formula $(w ; i, j)-(w-1 ; i, j)$.

Since that proof was written out I have endeavoured to obtain one that might be capable of being extended to the supposed analogous theorem, regarding pure reciprocants, expressed by the formula $(w ; i, j)-(w-1 ; i+1, j)$, but all my efforts and those of another and most skilful algebraist in this direction have hitherto proved ineffectual.

In aiming at this object, however, I obtained a second proof of Cayley's theorem, less compendious than the previous one, and subject to the drawback that it assumes the law of Reciprocity, but which possesses the advantage over it of being more direct and of looking the question, so to say, more squarely in the face. The forms of thought employed in it seem to me too peculiar and precious to be consigned to oblivion. I am not one of those who look upon Analysis as only valuable for the positive results to which it leads, and who regard proofs as almost a superfluity, thinking it sufficient that mathematical formulae should be obtained, no matter how, and duly entered on a register.

I look upon Mathematics not merely as a language, an art, and a science, but also as a branch of Philosophy, and regard the forms of reasoning which it embodies and enshrines as among the most valuable possessions of the human mind. Add to this that it is scarcely possible that a well-reasoned mathematical proof shall not contain within itself subordinate theoremsgerms of thought of intrinsic value and capable of extended application.

That such was the opinion of our High Pontiff is shown by the publication of his seven proofs of the Theorem of Reciprocity, a number to which subsequent researches have made almost annual additions (like so many continually augmenting asteroids in the Arithmetical Firmament) to such an extent that it would seem to be an interesting task for some one to undertake to form a corolla of these various proofs and to construct a reasoned bibliography, a catalogue raisonnée, of this one single theorem. For these reasons, I shall venture to put on record (valeat quantum) the following Second Proof of Cayley's Theorem.

The notation which I proceed to explain will be found very convenient. A rational integral homogeneous isobaric function will be called a gradient; its weight, degree, extent (extent meaning the number of letters after the first) will be denoted by $w ; i, j$ and spoken of as the type of the gradient. Either a single letter, such as $\phi$, will be employed to denote a gradient, or 
else its type enclosed in a parenthesis thus $[w ; i, j]$. The abbreviation $T \phi$ signifies the type of $\phi$; thus, $T \phi=w ; i, j$.

The number of terms in the most general gradient whose type is the same as that of $\phi$ will be spoken of as the denumerant of $\phi$. The letter $N$ will be used to denote such a denumerant; thus, $N \phi$ signifies the denumerant of $\phi$.

In like manner, the letter $\Delta$ will be used to denote the number of linear relations between the coefficients of any gradient, whenever such relations exist. Hence $N \phi-\Delta \phi$ expresses the number of terms in $\phi$ whose coefficients are left arbitrary. Obviously, when $\phi$ is the most general gradient of its type, we have

$$
\Delta \phi=0 \text {. }
$$

We also use $E$ to denote the $\ddot{j}-2 w$, which may be called the excess, of the gradient of type $w ; i, j$. Thus, if $T \phi=w ; i, j$, we write $E \phi=i j-2 w$.

The operators which we shall employ, namely, $\Omega$ and $\Omega^{\prime}$, are defined by the equations

$$
\begin{array}{lr}
\Omega=a_{0} \partial_{a_{1}}+a_{1} \partial_{a_{2}}+a_{2} \partial_{a_{3}}+\ldots, \\
\Omega^{\prime}= & a_{1} \partial_{a_{2}}+a_{2} \partial_{a_{3}}+\ldots .
\end{array}
$$

The first of these is of course an equivalent, but for present purposes more convenient, form of $a \partial_{b}+2 b \partial_{c}+3 c \partial_{d}+\ldots$, the ordinary invariant annihilator $\Omega$ (as will be evident on writing $\left.a_{0}=a, a_{1}=\frac{b}{1}, a_{2}=\frac{c}{1.2}, \ldots\right)$; the second of them, $\Omega^{\prime}$, is merely $\Omega$ deprived of its first term.

We may now give the following enunciation of the theorem to be proved:

If $\phi$ is the most general gradient of its type, $\Omega \phi$ is also the most general gradient of its type whenever $E \phi$ is not negative. In other words, we shall prove that, subject to the condition stated above, $\Delta \Omega \phi=0$ whenever $\Delta \phi=0$. This is equivalent to Cayley's Theorem on the number of linearly independent invariants. For the number of forms of the same type as $\phi$, and subject to annihilation by $\Omega$, is

$$
N \phi-N \Omega \phi+\Delta \Omega \phi
$$

and Cayley's Theorem states that the number of such forms is $N \phi-N \Omega \phi$, which will be the case when

$$
\Delta \Omega \phi=0 .
$$

The theorem of Reciprocity enables us to dispense with the discussion of those cases in which the extent $j$ is greater than the degree $i$. For since [Vol. III. of this Reprint, p. 151] the number of linearly independent invariants for the type $w ; j, i$ is the same as for the type $w ; i, j$, we can substitute the first of these types for the second, using $\psi$, whose type is $w ; j, i$, instead of $\phi$, whose type is $w ; i, j$. Thus we have

$$
N \psi-N \Omega \psi+\Delta \Omega \psi=N \phi-N \Omega \phi+\Delta \Omega \phi .
$$


But by Ferrers' proof of Euler's Theorem (vide "A Constructive Theory of Partitions" [p. 1, above]),

$$
N \psi=N \phi \text { and } N \Omega \psi=N \Omega \phi .
$$

It obviously follows that

$$
\Delta \Omega \psi=\Delta \Omega \phi .
$$

Cases for which the extent is greater than the degree may therefore be made to depend on those for which the degree is greater than the extent. Hence Cayley's Theorem depends on the proof that $\Delta \Omega \phi=0$ when $i=>j$ and $i j=>2 w$.

In the course of the demonstration, the following Lemma will be used:

$$
T \phi=w ; i, j \text { and } T \psi=i j-w ; i, j \text {, then } N \phi=N \psi \text {. }
$$

The types of the two gradients we are now considering may be said to be complementary, and then the Lemma may be enunciated in words as follows:

The denumerants of two gradients are equal when the types of the gradients are complementary.

The proof consists in showing that to each term of the type $w ; i, j$ there corresponds a term of the type $i j-w ; i, j$. Let $a_{0}{ }^{\lambda}{ }_{0} a_{1}{ }_{1}{ }_{1} a_{2}{ }^{\lambda_{2}} \ldots a_{j}{ }_{j}$ be any term of the type $w ; i, j$; then

and

$$
\begin{gathered}
w=\lambda_{1}+2 \lambda_{2}+3 \lambda_{3}+\ldots+j \lambda_{j} \\
i=\lambda_{0}+\lambda_{1}+\lambda_{2}+\lambda_{3}+\ldots+\lambda_{j} .
\end{gathered}
$$

Writing the suffixes of the letters $a_{0}, a_{1}, a_{2}, \ldots a_{j}$ in reverse order, everything else being kept unchanged, we obtain the term $a_{j}{ }_{0} a_{j-1}{ }_{1}{ }_{1} a_{j-2}{ }^{\lambda}{ }_{2} \ldots a_{0}{ }_{j}$, whose weight we will call $w^{\prime}$. Then

$$
\begin{aligned}
w^{\prime} & =j \lambda_{0}+(j-1) \lambda_{1}+(j-2) \lambda_{2}+\ldots+\lambda_{j-1} \\
& =j\left(\lambda_{0}+\lambda_{1}+\lambda_{2}+\ldots+\lambda_{j}\right)-\left(\lambda_{1}+2 \lambda_{2}+3 \lambda_{3}+\ldots+j \lambda_{j}\right) \\
& =\ddot{i}-w .
\end{aligned}
$$

The degree of the transformed term is still $i$, and its extent is still $j$, while its weight has become $i j-w$; its type is therefore complementary to that of the original term. Hence to each term of any given type there corresponds a term of the complementary type, and consequently the total number of possible terms (that is, the Denumerant) for each type is the same. Let

By means of this Lemma it can be shown that $\Delta \Omega \phi=0$ when $E \phi=-1$.

$$
T \phi=w ; i, j \text { where } i j-2 w=-1 \text {; }
$$

then, since $T \Omega \phi=w=1 ; i, j$, the types $T \phi$ and $T \Omega \phi$ are complementary (the sum of the weights being $w+w-1=i j$ ).

It follows from the Lemma that the Denumerants of $\phi$ and $\Omega \phi$ are equal. Hence

$$
\Delta \Omega \phi=0 .
$$


For if not, the number of independent terms in $\Omega \phi$ being less than the denumerant of $\Omega \phi$, will also be less than its equal, the denumerant of $\phi$, and therefore there will be one or more invariants of the type $w ; i, j$ for which the excess is negative. Since this is known to be impossible, we must have

$$
\Delta \Omega \phi=0 \text {. }
$$

We next prove that, in all cases for which $i=>w$, the number of linearly independent invariants of the type $w ; i, j$ is correctly given by the formula

$$
(w ; i, j)-(w-1 ; i, j)
$$

which is equivalent (as we showed at the beginning of Lecture XV.) to

$$
(w ; w, j)-(w-1 ; w, j)
$$

or, what is the same thing, to the coefficient of $a^{w} x^{w}$ in the expansion of

$$
F=\frac{1-x}{(1-a)(1-a x)\left(1-a x^{2}\right)\left(1-a x^{3}\right) \ldots\left(1-a x^{j}\right)} .
$$

Let the expansion of

be

$$
G=\frac{1-x}{(1-a x)\left(1-a x^{2}\right)\left(1-a x^{3}\right) \ldots\left(1-a x^{j}\right)}
$$

The expansion of $F$ is obtained by multiplying that of $G$ by the infinite geometrical series

$$
1+a+a^{2}+a^{3}+\ldots
$$

But we only require the coefficient of $a^{w} x^{w}$ in the expansion of $F$, so that we need only retain the portion

$$
A_{w} x^{w}\left(1+a+a^{2}+\ldots+a^{w}\right)
$$

of the above product instead of its complete expression.

It is of importance to notice here that $A_{w}$, which is independent of $x$, cannot contain any higher power of $a$ than $a^{w}$. (That this is so will be evident from the constitution of the fraction $G$, for clearly no power of $a$ in the expansion of $G$ can be associated with a lower power of $x$.) Thus we see that

$$
A_{w}=\alpha a^{w}+\beta a^{w-1}+\gamma a^{w-2}+\ldots+\kappa a+\lambda,
$$

and consequently

$$
A_{w} x^{w}\left(1+a+a^{2}+\ldots+a^{w}\right)=\ldots+a^{w} x^{w}(\alpha+\beta+\gamma+\ldots+\kappa+\lambda)+\ldots .
$$

Hence the coefficient of $a^{w} x^{w}$ in the expansion of $F$ is

$$
\alpha+\beta+\gamma+\ldots+\kappa+\lambda,
$$

which is the value assumed by $A_{w}$ when in it we write $a=1$. Call this value $A_{w}{ }^{\prime}$, and let the value of $G$ when $a=1$ be denoted by $G^{\prime}$. Then $A_{w}{ }^{\prime}$ is the coefficient of $x^{w}$ in

$$
G^{\prime}=\frac{1}{\left(1-x^{2}\right)\left(1-x^{3}\right) \ldots\left(1-x^{j}\right)} .
$$


Hence we see that, when $i=>w$, the value of $(w ; i, j)-(w-1 ; i, j)$ is the total number of ways in which $w$ can be made up of the parts $2,3, \ldots j$.

We have yet to show that this number is the same as that of the linearly independent invariants of the type $w ; i, j$ when $i=>w$.

This follows from the known theorem that every invariant is either a rational integral function of the Protomorphs $a, P_{2}, P_{3}, \ldots P_{j}$ (meaning the invariant $a$ and those of the second and third degrees alternately whose first terms are $a c, a^{2} d$, ae, $\left.a^{2} f, \ldots\right)$, or can be made so by multiplying it by a suitable power of $a$. Thus, if $I$ be any invariant of degree $i$ and weight $w$,

$$
I a^{w-i}=\Phi\left(a, P_{2}, P_{3}, \ldots P_{j}\right)
$$

where $\Phi$, which is of degree-weight $w . w$ when expressed in terms of $a, b, c, \ldots$, is rational and integral as regards the protomorphs.

When $i=>w$, writing

$$
I=a^{i-w} \Phi\left(a, P_{2}, P_{3}, \ldots P_{j}\right),
$$

$\Phi$ consists of a series of terms of the form $A a^{\theta} P_{2}{ }^{\lambda} P_{3}{ }^{\mu} \ldots P_{j}{ }^{\rho}$, each with an arbitrary coefficient, where, since

$$
2 \lambda+3 \mu+4 \nu+\ldots+j \rho=w
$$

the number of arbitrary constants in $\Phi$ is the total number of partitions of $w$ into parts $2,3, \ldots j$. Hence the number of linearly independent invariants of the type $w ; i, j$ is also this number of partitions, that is, by what precedes is $(w ; i, j)-(w-1 ; i, j)$. This proves Cayley's theorem for cases in which $i=>w$.

But when $i<w$, the equation

$$
I a^{w-i}=\Phi\left(a, P_{2}, P_{3}, \ldots P_{j}\right)
$$

shows that the coefficients of $\Phi$ are not all arbitrary, but must be so chosen that $\Phi$ may be divisible by $a^{w-i}$, and the reasoning employed in the case of $i=>w$ no longer holds.

It will be convenient at this point of the investigation to review the results we have hitherto obtained and to see what remains to be proved.

Cayley's Theorem has been demonstrated for cases in which the degree is not less than the weight. This will be expressed by saying that

$$
\Delta \Omega[w ; i, j]=0 \text { when } i=>w .
$$

We have also proved that

$$
\Delta \Omega[w ; i, j]=0 \text { when } \ddot{j}-2 w=-1 .
$$

The law of reciprocity has been expressed in the form

$$
\Delta \Omega[w ; i, j]=\Delta \Omega[w ; j, i],
$$

where $[w ; i, j]$ denotes the most general gradient of the type $w ; i, j$. 
The theorem to be proved is that

$$
\Delta \Omega[w ; i, j]=0 \text { when } i j-2 w=>0 ;
$$

but we may at once dismiss those cases in which $i=>w$, and (assuming the theorem to have been proved for Quantics of order inferior to $j$ ) those in which $i<j$, for these depend on the truth of the theorem for a Quantic of order $i$.

It remains, then, to prove that, when $i j-2 w=>0, \Delta \Omega[w ; i, j]=0$ for values of $i$ inferior to $w$, but not inferior to $j$. This may be effected as follows :

Let $\phi$ be the most general gradient of the type $w ; i+1, j$, and suppose

$$
\phi=P+Q a+R a^{2}+S a^{3},
$$

where $P, Q$ and $R$ do not contain the letter $a$, though $S$ may do so. Then, writing

$$
\phi_{1}=Q+R a+S a^{2}
$$

$\phi_{1}$ is the most general gradient of the type $w ; i, j$.

Now, if $\Omega=a \partial_{b}+b \partial_{c}+c \partial_{d}+\ldots$, and $\Omega^{\prime}=b \partial_{c}+c \partial_{d}+\ldots$, we have

$$
\Omega \phi=\Omega^{\prime} P+\left(\Omega^{\prime} Q+\frac{d P}{d b}\right) a+\left(\Omega^{\prime} R+\frac{d Q}{d b}\right) a^{2}+\left(\Omega S+\frac{d R}{d b}\right) a^{3},
$$

and

$$
\Omega \phi_{1}=\Omega^{\prime} Q+\left(\Omega^{\prime} R+\frac{d Q}{d b}\right) a+\left(\Omega S+\frac{d R}{d b}\right) a^{2}
$$

Confining our attention for the present to $\Omega \phi_{1}$, it is clear that if no linear relations exist among the coefficients of $\Omega^{\prime} R$ (that is, if $\Delta \Omega^{\prime} R=0$ ) the coefficients of $\Omega^{\prime} Q$ are not connected with those of $\Omega^{\prime} R+\frac{d Q}{d b}$ by any linear relation. For the coefficient of each term of $\Omega^{\prime} R+\frac{d Q}{d b}$ is the sum of a single coefficient of $Q$ and an independent linear function of the coefficients of $R$. Moreover, obviously the coefficients of $\Omega^{\prime} Q$ are unconnected with those of $\Omega S+\frac{d R}{d b}$.

If, then, the coefficients of $\Omega^{\prime} Q$ are not related inter se (that is, if $\left.\Delta \Omega^{\prime} Q=0\right)$, we have

$$
\Delta \Omega \phi_{1}=\Delta\left\{\left(\Omega^{\prime} R+\frac{d Q}{d b}\right) a+\left(\Omega S+\frac{d R}{d b}\right) a^{2}\right\} .
$$

Looking now to the expression (1) for $\Omega \phi$, we see immediately from (2) that any linear relation subsisting between the coefficients of $\Omega \phi_{1}$ will also subsist between those of $\Omega \phi$, and therefore that $\Delta \Omega \phi_{1}$ is not greater than $\Delta \Omega \phi$.

If, then, $\Delta \Omega \phi=0$, it follows that $\Delta \Omega \phi_{1}=0$, provided that both the supplementary conditions $\Delta \Omega^{\prime} Q=0$ and $\Delta \Omega^{\prime} R=0$ are also satisfied. 
Now, since $\phi_{1}=Q+R a+S a^{2}$ is the most general gradient of the type $w ; i, j$,

$Q$ will be the most general gradient of the type $w-i ; i, j-1$, and $R$

$$
w-i+1 ; i-1, j-1
$$

when in $Q$ and $R$ we change $b, c, d, \ldots$ into $a, b, c, \ldots$ This change converts $\Omega^{\prime}=b \partial_{c}+c \partial_{d}+\ldots$ into $\Omega=a \partial_{b}+b \partial_{c}+\ldots$. Hence the conditions $\Delta \Omega^{\prime} Q=0$ and $\Delta \Omega^{\prime} R=0$ are respectively equivalent to

$$
\Delta \Omega[w-i ; i, j-1]=0 \text { and } \Delta \Omega[w-i+1 ; i-1, j-1]=0 .
$$

Supposing these supplementary conditions to be satisfied, what we have proved is that when

$$
\begin{array}{ll} 
& \Delta \Omega[w ; i+1, j]=0(\text { that is, } \Delta \Omega \phi=0), \\
\text { then also } & \Delta \Omega[w ; i, j]=0\left(\text { that is, } \Delta \Omega \phi_{1}=0\right) .
\end{array}
$$

Now,

$$
\begin{aligned}
& T \phi=w ; i+1, j, \text { so that } E \phi=(i+1) j-2 w=(i j-2 w)+j, \\
& T Q=w-i ; i, j-1, \text { so that } E Q=i(j-1)-2(w-i)=(i j-2 w)+i, \\
& T R=w-i+1 ; i-1, j-1, \text { so that } E R=(i-1)(j-1)-2(w-i+1) \\
& =(i j-2 w)+i-j-1 .
\end{aligned}
$$

Thus, when $i j-2 w=>0$ and $i=>j$,

$E \phi$ and $E Q$ are both positive.

$E R$ is in general $=>0$, but in the special case where $i j-2 w=0$ and $i=j$, we have $E R=-1$. Except in this case (which gives us no trouble, since we have seen that $\Delta \Omega R=0$ in consequence of $E R=-1$ ), we have never to deal with a type of which the excess is negative.

Hence, if we assume Cayley's Theorem to have been proved for all extents up to $j-1$ inclusive, we have-

and

$$
\Delta \Omega[w-i ; i, j-1]=0,
$$

$$
\Delta \Omega[w-i+1 ; i-1, j-1]=0,
$$

(that is, the two supplementary conditions are satisfied).

We wish to extend the theorem to the extent $j$.

Subject to the conditions $i=>j$ and $i j-2 w=>0$, we have

$$
\Delta \Omega[w ; i, j]=0 \text { if } \Delta \Omega[w ; i+1, j]=0 .
$$

But we need consider no value of $i$ greater than $w$, as we have proved that

therefore

$$
\begin{aligned}
& \Delta \Omega[w ; w, j]=0=\Delta \Omega[w ; w+\kappa, j] \\
& \Delta \Omega[w ; w-1, j]=0, \\
& \Delta \Omega[w ; w-2, j]=0, \\
& \cdots \ldots \ldots \ldots \ldots \cdots \cdots \cdots \\
& \Delta \Omega[w ; j, j] \quad=0 .
\end{aligned}
$$


As previously shown, the theorem is true for all values of $i$ inferior to $j$ if it is true for all Quantics of inferior order. Thus the theorem is true for a Quantic of order $j$ and for every value of $i$ if it is true for all Quantics of order inferior to $j$. But it is true for the Quadric (where $j=2$ )*; therefore also for the Cubic $(j=3)$; therefore also for the Quartic $(j=4)$, and so universally. Hence the theorem to be proved is demonstrated.

\section{LECTURE XXVIII.}

We now resume the theory of Principiants and proceed to prove the important theorem that every Principiant is either simply an invariant in respect to a known series of pure reciprocants, which we call $A, B, C, D, \ldots$, or else becomes such an invariant'when multiplied by $a^{w-i}$, where $w$ is the weight and $i$ the degree of the Principiant in question. Using the letter $M$ to denote the pure reciprocant $a c-\frac{5}{4} b^{2}$, and $G$ the ordinary eductive generator,

$$
4\left(a c-b^{2}\right) \partial_{b}+5(a d-b c) \partial_{c}+6(a e-b d) \partial_{d}+7(a f-b e) \partial_{e}+\ldots
$$

(which, it will be remembered, is only another form of $a \frac{d}{d x}-\mu b$, with the advantage of the $\mu$ being suppressed, that is, only implicitly contained), we obtain in succession the values of $A, B, C, D, \ldots$ from the following equations:

$$
\begin{aligned}
& 5 A=G M, \\
& 6 B=G A, \\
& 7 C=G B-M A, \\
& 8 D=G C-2 M B, \\
& 9 E=G D-3 M C,
\end{aligned}
$$

On performing the calculations indicated by these equations we shall find

$$
\begin{aligned}
& A=a^{2} d-3 a b c+2 b^{3} \\
& B=a^{3} e-2 a^{2} c^{2}-\frac{7}{2} a^{2} b d+\frac{17}{2} a b^{2} c-4 b^{4} \\
& C=a^{4} f-5 a^{3} c d-4 a^{3} b e+13 a^{2} b c^{2}+\frac{45}{4} a^{2} b^{2} d-\frac{103}{4} a b^{3} c+\frac{19}{2} b^{5} \\
& D=a^{5} g-\frac{25}{8} a^{4} d^{2}-6 a^{4} c e+7 a^{3} c^{3}+\text { terms involving } b \\
& E=a^{6} h-\frac{15}{2} a^{5} d e-7 a^{5} c f+29 a^{4} c^{2} d+\text { terms involving } b
\end{aligned}
$$

* When $j=2$ the condition $i j=>2 w$ becomes identical with $i=>w$; but we have already seen that the theorem is true whenever $i=>w$.

s. IV. 
The fact that $D$ is a pure reciprocant enables us to calculate the terms in $E$ which are independent of $b$ without a previous knowledge of the values of those terms in $D$ which involve $b$. For, since

$$
\begin{aligned}
& G=4\left(a c-b^{2}\right) \partial_{b}+\ldots \text { and } V=2 a^{2} \partial_{b}+\ldots, \\
& a^{2} G-2\left(a c-b^{2}\right) V \text { does not contain } \partial_{b} .
\end{aligned}
$$

Hence the operation of $a^{2} G-2\left(a c-b^{2}\right) V$ on terms involving $b$ cannot give rise to terms independent of $b$. But,

$D$ being a pure reciprocant, $V D=0$;

so that

$$
\left\{a^{2} G-2\left(a c-b^{2}\right) V\right\} D=a^{2} G D,
$$

and the terms of $a^{2} G D$ which do not involve $b$ are found by operating with

$$
\left[a^{2} G-2\left(a c-b^{2}\right) V\right]_{b=0}
$$

on the terms of $D$ which do not involve $b$.

If, now, we use $M_{0}, A_{0}, B_{0}, C_{0}, \ldots$ to denote those portions of $M, A, B, C, \ldots$ which are independent of $b$, and write

$$
\left[a^{2} G-2\left(a c-b^{2}\right) V\right]_{b=0}=a^{2} G_{0}
$$

we shall still have

$$
9 E_{0}=G_{0} D_{0}-3 M_{0} C_{0}
$$

and in general the law of successive derivation for $A_{0}, B_{0}, C_{0}, D_{0}, \ldots$ is the same as that for $A, B, C, D, \ldots$ except that $G_{0}$ takes the place of $G$.

We have

$$
\begin{aligned}
a^{2} G_{0} & =\left[a^{2} G-2\left(a c-b^{2}\right) V\right]_{b=0} \\
& =a^{2}\left(5 a d \partial_{c}+6 a e \partial_{d}+7 a f \partial_{e}+8 a g \partial_{f}+9 a h \partial_{g}+\ldots\right) \\
& -2 a c\left\{6 a c \partial_{d}+7 a d \partial_{e}+\left(8 a e+4 c^{2}\right) \partial_{f}+(9 a f+9 c d) \partial_{g}+\ldots\right\}
\end{aligned}
$$

so that

$$
\begin{aligned}
G_{0}=5 a d \partial_{c}+6\left(a e-2 c^{2}\right) \partial_{d} & +7(a f-2 c d) \partial_{e} \\
& +\frac{8}{a}\left(a^{2} g-2 a c e-c^{3}\right) \partial_{f}+\frac{9}{a}\left(a^{2} h-2 a c f-2 c^{2} d\right) \partial_{g}+\ldots
\end{aligned}
$$

and consequently (since $M_{0}=a c$ ),

$$
\begin{array}{lll}
5 A_{0}=G_{0} M_{0} & \text { gives } & A_{0}=a^{2} d, \\
6 B_{0}=G_{0} A_{0} & \# & B_{0}=a^{3} e-2 a^{2} c^{2}, \\
7 C_{0}=G_{0} B_{0}-M_{0} A_{0} & \# & C_{0}=a^{4} f-5 a^{3} c d, \\
8 D_{0}=G_{0} C_{0}-2 M_{0} B_{0} & \# & D_{0}=a^{5} g-\frac{25}{8} a^{4} d^{2}-6 a^{4} c e+7 a^{3} c^{3}, \\
9 E_{0}=G_{0} D_{0}-3 M_{0} C_{0} & \# & E_{0}=a^{6} h-\frac{15}{2} a^{5} d e-7 a^{5} c f+29 a^{4} c^{2} d,
\end{array}
$$


Thus, for example,

$$
\begin{aligned}
8 D_{0} & =G_{0}\left(a^{4} f-5 a^{3} c d\right)-2 a c\left(a^{3} e-2 a^{2} c^{2}\right) \\
& =-25 a^{4} d^{2}-30 a^{3} c\left(a e-2 c^{2}\right)+8 a^{3}\left(a^{2} g-2 a c e-c^{3}\right)-2 a c\left(a^{3} e-2 a^{2} c^{2}\right) ;
\end{aligned}
$$

whence

$$
D_{0}=a^{5} g-\frac{25}{8} a^{4} d^{2}-6 a^{4} c e+7 a^{3} c^{3}
$$

Again, $9 E_{0}=G_{0}\left(a^{5} g-\frac{25}{8} a^{4} d^{2}-6 a^{4} c e+7 a^{3} c^{3}\right)-3 a c\left(a^{4} f-5 a^{3} c d\right)$

$$
\begin{gathered}
=5 a d\left(-6 a^{4} e+21 a^{3} c^{2}\right)-\frac{75}{2}\left(a e-2 c^{2}\right) a^{4} d-42(a f-2 c d) a^{4} c \\
+9\left(a^{2} h-2 a c f-2 c^{2} d\right) a^{4}-3 a c\left(a^{4} f-5 a^{3} c d\right),
\end{gathered}
$$

gives

$$
E_{0}=a^{6} h-\frac{15}{2} a^{5} d e-7 a^{5} c f+29 a^{4} c^{2} d
$$

Similarly, from the known values of $D_{0}$ and $E_{0}$ we may deduce that of the next letter, $F_{0}$, and so on to any extent.

It may be noticed that each of the pure reciprocants $A, B, C, D, \ldots$ can be determined without ambiguity, by means of the annihilator $V$, when the portions of them, $A_{0}, B_{0}, C_{0}, D_{0}, \ldots$ independent of $b$ are known.

For suppose $R$ and $R^{\prime}$ to be two reciprocants, of weight $w$, for each of which the terms independent of $b$ are the same. Then their difference is divisible by $b$. Let

$$
R-R^{\prime}=b \phi ; \text { then } V(b \phi)=0 ; \text { that is, } 2 a^{2} \phi+b V \phi=0 .
$$
Then

Hence $\phi$ is divisible by $b$, and $R-R^{\prime}$ is divisible by $b^{2}$; say $R-R^{\prime}=b^{2} \psi$.

$$
V\left(b^{2} \psi\right)=4 a^{2} b \psi+b^{2} V \psi=0
$$

showing that $\psi$ is divisible by $b$, and $R-R^{\prime}$ by $b^{3}$.

By continually reasoning in this manner, we prove that $R-R^{\prime}$ must be divisible by $b^{w}$; and then the remaining factor (being of weight 0 ) is necessarily of the form $\lambda a^{\theta}$, where $\lambda$ and $\theta$ are numerical constants. Thus

$$
R-R^{\prime}=\lambda a^{\theta} b^{w} \text {, and consequently } V\left(\lambda a^{\theta} b^{w}\right)=0 .
$$

This is impossible unless $\lambda=0$, when the two reciprocants $R, R^{\prime}$ become equal, showing that there cannot be two different reciprocants for which the terms independent of $b$ are the same. When, therefore, the terms which do not involve $b$ of any pure reciprocant are known, the complete expression of that reciprocant can be determined without ambiguity.

Each reciprocant of the series $A, B, C, D, \ldots$ possesses the property of being, so to say, an Invariant relative to the one which precedes it, meaning that the operation of $\Omega=a \partial_{b}+2 b \partial_{c}+3 c \partial_{d}+\ldots$ on any letter gives (to a 
factor près) the one immediately preceding it. The first letter, $A$, is an Invariant in the ordinary sense. We can in fact show that

$$
\begin{aligned}
& \Omega A=0, \\
& \Omega B=A \times \frac{a}{2}, \\
& \Omega C=2 B \times \frac{a}{2}, \\
& \Omega D=3 C \times \frac{a}{2}, \\
& \Omega E=4 D \times \frac{a}{2},
\end{aligned}
$$

The proof depends on a formula established in Lecture XXVI. of this course [p. 457, above], namely

$$
\Omega G-G \Omega=w a-b \Omega \text {, }
$$

where $G$ is the generator $4\left(a c-b^{2}\right) \partial_{b}+5(a d-b c) \partial_{c}+\ldots$, and $w$ is the weight of the operand.

Thus, observing that the weights of $A, B, C, D, \ldots$ are $3,4,5,6, \ldots$ respectively, we have

$$
\begin{aligned}
& (\Omega G-G \Omega) A=(3 a-b \Omega) A \\
& (\Omega G-G \Omega) B=(4 a-b \Omega) B \\
& (\Omega G-G \Omega) C=(5 a-b \Omega) C
\end{aligned}
$$

Now, since $A$ is the well-known invariant $a^{2} d-3 a b c+2 b^{3}$, we may write $\Omega A=0$ in the first of these equations, which then reduces to

But, since

$$
\Omega G A=3 a A \text {. }
$$

we have

$$
\begin{aligned}
6 B & =G A, \\
6 \Omega B & =\Omega G A=3 a A .
\end{aligned}
$$

Thus

$$
\Omega B=A \times \frac{a}{2} .
$$

Again, substituting for $\Omega B$ in the formula

$$
(\Omega G-G \Omega) B=(4 a-b \Omega) B,
$$

we find

$$
\Omega G B-G\left(\frac{a A}{2}\right)=4 a B-\frac{a b}{2} A,
$$

where, since $G$ (which is linear in $\partial_{b}, \partial_{c}, \ldots$ and does not contain $\partial_{a}$ ) does not operate on $a$,

$$
\begin{aligned}
& G\left(\frac{a A}{2}\right)=\frac{a}{2} G A=3 a B, \\
& \text { and consequently } \quad \Omega G B+\frac{a b}{2} A=7 a B \text {. }
\end{aligned}
$$


Now,

$$
7 C=G B-M A
$$

so that

$$
7 \Omega C=\Omega G B-A \Omega M-M \Omega A .
$$

But, since

$$
\begin{aligned}
& \Omega M=\Omega\left(a c-\frac{5 b^{2}}{4}\right)=-\frac{a b}{2} \text { and } \Omega A=0, \\
& 7 \Omega C=\Omega G B+\frac{a b}{2} A=7 a B .
\end{aligned}
$$

Thus

$$
\Omega C=2 B \times \frac{a}{2} .
$$

We may, in exactly the same way, prove that

and so on to any extent.

$$
\begin{aligned}
& \Omega D=3 C \times \frac{a}{2}, \\
& \Omega E=4 D \times \frac{a}{2},
\end{aligned}
$$
letters

In the following inductive proof it will be convenient to denote the

by

$$
\begin{gathered}
A, B, C, D, E, \ldots \\
u_{0}, u_{1}, u_{2}, u_{3}, u_{4}, \ldots,
\end{gathered}
$$

and then the theorem to be proved is that

$$
\Omega u_{n}=n u_{n-1} \times \frac{a}{2} \text {. }
$$

When this notation is used, the law of successive derivation which defines the capital letters is expressed by the equation

$$
(n+7) u_{n+2}-G u_{n+1}+(n+1) M u_{n}=0,
$$

where $G$ is the generator

$$
4\left(a c-b^{2}\right) \partial_{b}+5(a d-b c) \partial_{c}+\ldots, \text { and } M=a c-\frac{5 b^{2}}{4}
$$

Operating with $\Omega$ on the above equation, we obtain

$$
(n+7) \Omega u_{n+2}-\Omega G u_{n+1}+(n+1)\left(M \Omega u_{n}+u_{n} \Omega M\right)=0 .
$$

Now, the weights of $u_{0}, u_{1}, u_{2}, \ldots$ are $3,4,5, \ldots$ respectively, and consequently the operation of

$$
\Omega G-G \Omega=w a-b \Omega
$$

on $u_{n+1}$ (whose weight is $n+4$ ) gives

$$
(\Omega G-G \Omega) u_{n+1}=(n+4) a u_{n+1}-b \Omega u_{n+1} .
$$

Or, assuming that $\Omega u_{\kappa}=\kappa u_{\kappa-1} \times \frac{a}{2}$ for all values of $\kappa$ as far as $n+1$ inclusive (it has previously been shown that $\Omega B=A \times \frac{a}{2}$ and $\Omega C=2 B \times \frac{a}{2}$, so that the theorem is true for $\kappa=1$ and $\kappa=2$ ),

$$
\begin{aligned}
\Omega G u_{n+1} & =G \Omega u_{n+1}+(n+4) a u_{n+1}-b \Omega u_{n+1} \\
& =(n+1) G\left(\frac{a}{2} u_{n}\right)+(n+4) a u_{n+1}-(n+1) \frac{a b}{2} u_{n} .
\end{aligned}
$$


But (remembering that $G$ does not operate on $a$, so that $G \cdot \frac{a}{2} u_{n}=\frac{a}{2} G u_{n}$ ) we have, in virtue of equation (1),

$$
G\left(\frac{a}{2} u_{n}\right)=\frac{a}{2}\left\{(n+6) u_{n+1}+n M u_{n-1}\right\} .
$$

Hence it follows that

$$
\begin{aligned}
\Omega G u_{n+1} & =\frac{n+1}{2} a\left\{(n+6) u_{n+1}+n M u_{n-1}\right\}+(n+4) a u_{n+1}-(n+1) \frac{a b}{2} u_{n} \\
& =\frac{(n+2)(n+7)}{2} a u_{n+1}+\frac{n(n+1)}{2} a M u_{n-1}-(n+1) \frac{a b}{2} u_{n} .
\end{aligned}
$$

On substituting this in (2) we obtain

$$
\begin{aligned}
& (n+7)\left\{\Omega u_{n+2}-(n+2) \frac{a}{2} u_{n+1}\right\} \\
& +(n+1) M\left\{\Omega u_{n}-n \frac{a}{2} u_{n-1}\right\} \\
& +(n+1) u_{n}\left\{\Omega M+\frac{a b}{2}\right\}=0
\end{aligned}
$$

This reduces to

$$
\Omega u_{n+2}=(n+2) \frac{a}{2} u_{n+1} \text {. }
$$

For, according to the assumption previously made in the course of the demonstration,

$$
\Omega u_{n}=n \frac{a}{2} u_{n-1}
$$

so that the second term vanishes; and the third term vanishes because

$$
\Omega M=\Omega\left(a c-\frac{\check{5} b^{2}}{4}\right)=-\frac{a b}{2} .
$$

We have therefore proved that if the theorem is true for $\Omega u_{\kappa}$, when $\kappa$ has any value up to $n+1$ inclusive, it is also true for $\Omega u_{n+2}$. But the theorem holds for $\kappa=1$, and for $\kappa=2$. It therefore holds universally for any positive integer value of $\kappa$.

Recalling the known values of the reciprocants $M, A, B, C, D, \ldots$ we observe that their principal terms are $a c, a^{2} d, a^{3} e, a^{4} f, a^{5} g, \ldots$, where it is to be noticed that the most advanced of the small letters in the expression for any capital letter occurs only in the first degree multiplied by a power of a. In other words, $M, A, B, C, D, \ldots$ form a series of Protomorphs, and consequently every Pure Reciprocant can, as we have already seen (vide [p. 384, above]), be expressed as a function of $a, M, A, B, C, D, \ldots$ rational in all of them and integral in all except $a$. 
But it is further to be noticed that whereas

$\begin{array}{ccccc}a \text { is of degree } & 1 \text { and weight } & 0, \\ M & \# & 2 & \# & 2, \\ A & \# & 3 & \# & 3, \\ B & \# & 4 & \# & 4,\end{array}$

and in fact that every capital letter is of equal weight and degree.

From this it will follow that every Pure Reciprocant will be the product of a power of $a$ into a function of the capital letters alone.

For let $i$ be the degree and $w$ the weight of any pure reciprocant expressed in terms of $a, M, A, B, C, \ldots$, and suppose one of its terms to be

$$
a^{\eta} M^{\theta} A^{\wedge} B^{\wedge} C^{\mu} \ldots \text {; }
$$

then

$$
\begin{array}{r}
\eta+2 \theta+3 \kappa+4 \lambda+5 \mu+\ldots=i \\
2 \theta+3 \kappa+4 \lambda+5 \mu+\ldots=w .
\end{array}
$$

and

Hence

$$
\eta=i-w
$$

which is the same for every term of the pure reciprocant in question. Thus each term contains $a^{i-w}$ as a factor, and the reciprocant is of the form

$$
a^{i-w} \Phi(M, A, B, C, D, \ldots) \text {. }
$$

Let us now consider any Principiant $P$; since $P$ is a pure reciprocant, we must have

$$
P=a^{i-w} \Phi(M, A, B, C, D, \ldots) .
$$

But Principiants are subject to annihilation by $\Omega$, and consequently $\Omega P=0$, which gives

$$
\frac{d \Phi}{d M} \Omega M+\frac{d \Phi}{d A} \Omega A+\frac{d \Phi}{d B} \Omega B+\frac{d \Phi}{d C} \Omega C+\ldots=0
$$

On writing for

$\Omega M, \quad \Omega A, \quad \Omega B, \quad \Omega C, \ldots$

their values

$$
-b \times \frac{a}{2}, \quad 0, \quad A \times \frac{a}{2}, 2 B \times \frac{a}{2}, \ldots
$$

we obtain

$$
\frac{a}{2}\left(-b \partial_{M}+A \partial_{B}+2 B \partial_{C}+3 C \partial_{D}+\ldots\right) \Phi=0
$$

From this it would follow that $\Phi$ is an invariant in the two sets of letters

$$
-b, M \text { and } A, B, C, D, \ldots \text {; }
$$

but it is easy to see that it is an invariant in the latter set exclusively. For $M$ and $A, B, C, D, \ldots$ being all of them pure reciprocants,

$$
\Phi \text { and } \partial_{M} \Phi, \partial_{B} \Phi, \partial_{C} \Phi, \partial_{D} \Phi, \ldots
$$

which are functions of $M, A, B, C, \ldots$ exclusively, must also be pure reciprocants. 
If, then, we operate with $V$ on

$$
\left(-b \partial_{M}+A \partial_{B}+2 B \partial_{C}+3 C \partial_{D}, \ldots\right) \Phi=0
$$

we shall find $V\left(-b \partial_{M}\right) \Phi=0$ (every other term being annihilated by $V$ ). Thus

$$
V\left(b \partial_{M}\right) \Phi=\left(\partial_{M} \Phi\right) V b=2 a^{2} \partial_{M} \Phi=0,
$$

and consequently $\partial_{M} \Phi=0$. Hence

$$
\left(A \partial_{B}+2 B \partial_{C}+3 C \partial_{D}+\ldots\right) \Phi=0 .
$$

The equation $\partial_{M} \Phi=0$ shows that $M$ does not appear in the expression for any principiant in terms of the capital letters, while

$$
\left(A \partial_{B}+2 B \partial_{C}+3 C \partial_{D}+\ldots\right) \Phi=0
$$

shows that $\Phi$ is an invariant in $A, B, C, D, \ldots$

We have thus shown that every invariant of

$$
(A, B, C, \ldots)(x, y)^{j}
$$

is a principiant, and conversely that every principiant is an invariant of

$$
(A, B, C, \ldots)(x, y)^{j},
$$

or such an invariant multiplied by a power of $a$.

\section{LECTURE XXIX.}

From the theorem that every Principiant is (to a power of a près) an Invariant in the reciprocantive elements $A, B, C, \ldots$ we readily deduce its correlative in which, everything else remaining unchanged, the reciprocantive elements $A, B, C, \ldots$ are replaced by a set of invariantive elements which we call $A_{0}, A_{1}, A_{2}, \ldots$. The equations connecting the new elements with the old ones are as follows:

$$
\begin{aligned}
& A_{0}=A \\
& A_{1}=B-\left(\frac{b}{2}\right) A \\
& A_{2}=C-2\left(\frac{b}{2}\right) B+\left(\frac{b}{2}\right)^{2} A \\
& A_{3}=D-3\left(\frac{b}{2}\right) C+3\left(\frac{b}{2}\right)^{2} B-\left(\frac{b}{2}\right)^{3} A \\
& A_{4}=E-4\left(\frac{b}{2}\right) D+6\left(\frac{b}{2}\right)^{2} C-4\left(\frac{b}{2}\right)^{3} B+\left(\frac{b}{2}\right)^{4} A
\end{aligned}
$$


We have, in the first place, to prove that $A_{0}, A_{1}, A_{2}, \ldots$ are all of them invariants in the small letters $a, b, c, \ldots$ This is an immediate consequence of the identities

$$
\begin{aligned}
& \Omega A=0 \\
& \Omega B=A \times \frac{a}{2}, \\
& \Omega C=2 B \times \frac{a}{2},
\end{aligned}
$$

established in the preceding Lecture, coupled with the fact that $\Omega b=a$. Thus

$$
\begin{aligned}
& \Omega A_{0}=\Omega A=0 \\
& \Omega A_{1}=-\frac{b}{2} \Omega A+\left(\Omega B-A \times \frac{a}{2}\right)=0 \\
& \Omega A_{2}=\left(\frac{b}{2}\right)^{2} \Omega A-2\left(\frac{b}{2}\right)\left(\Omega B-A \times \frac{a}{2}\right)+\left(\Omega C-2 B \times \frac{a}{2}\right)=0
\end{aligned}
$$

and in general, writing the equation which gives $A_{n}$ in the form

$$
\begin{aligned}
A_{n}=\left(-\frac{b}{2}\right)^{n} A+n\left(-\frac{b}{2}\right)^{n-1} B & \frac{n(n-1)}{1.2}\left(-\frac{b}{2}\right)^{n-2} C \\
& +\frac{n(n-1)(n-2)}{1.2 .3}\left(-\frac{b}{2}\right)^{n-3} D+\ldots
\end{aligned}
$$

and operating on it with $\Omega$, we find

$$
\begin{aligned}
\Omega A_{n}= & \left(-\frac{b}{2}\right)^{n} \Omega A+n\left(-\frac{b}{2}\right)^{n-1}\left(\Omega B-A \times \frac{a}{2}\right) \\
& +\frac{n(n-1)}{1.2}\left(-\frac{b}{2}\right)^{n-2}\left(\Omega C-2 B \times \frac{a}{2}\right) \\
& +\frac{n(n-1)(n-2)}{1.2 .3}\left(-\frac{b}{2}\right)^{n-3}\left(\Omega D-3 C \times \frac{a}{2}\right)+\ldots \\
= & 0 \text { (each term vanishing separately). }
\end{aligned}
$$

We next observe that

$$
\left(A_{0}, A_{1}, A_{2}, \ldots\right)(x, y)^{j} \text {, being equal to }(A, B, C, \ldots)\left(x-\frac{b}{2} y, y\right)^{j} \text {, }
$$

is a linear transformation of $(A, B, C, \ldots)(x, y)^{j}$, and that the determinant of the transformation $\left|\begin{array}{rr}1 & -\frac{b}{2} \\ 0 & 1\end{array}\right|$ is equal to unity.

Hence every invariant in $A_{0}, A_{1}, A_{2}, \ldots$ is equal to the corresponding invariant in $A, B, C, \ldots$, which proves the theorem in question. 
Each of the invariantive elements $A_{0}, A_{1}, A_{2}, \ldots$ is, so to say, a reciprocant relative to the one which immediately precedes it, just as in the cognate theorem each of the capital letters $A, B, C, \ldots$ was an invariant relative to its antecedent. It is in fact easily seen that

and in general

$$
\begin{aligned}
& V A_{0}=0, \\
& V A_{1}=-A_{0} a^{2}, \\
& V A_{2}=-2 A_{1} a^{2}, \\
& V A_{3}=-3 A_{2} a^{2}, \\
& \cdots \cdots \cdots \cdots \cdots \cdots \\
& V A_{n}=-n A_{n-1} a^{2} .
\end{aligned}
$$

Thus, for example, if we operate with $V$ on

$$
A_{3}=D-3\left(\frac{b}{2}\right) C+3\left(\frac{b}{2}\right)^{2} B-\left(\frac{b}{2}\right)^{3} A
$$

remembering that $A, B, C, D$ are pure reciprocants, we shall find

$$
V A_{3}=-\frac{3}{2}\left\{C-2\left(\frac{b}{2}\right) B+\left(\frac{b}{2}\right)^{2} A\right\} V B
$$

But

$$
C-2\left(\frac{b}{2}\right) B+\left(\frac{b}{2}\right)^{2} A=A_{2} \text { and } V b=2 a^{2}
$$

so that

$$
V A_{3}=-3 A_{2} a^{2} \text {. }
$$

In like manner, operating with $V$ on

$$
A_{n}=(A, B, C, \ldots)\left(-\frac{b}{2}, 1\right)^{n}
$$

we obtain

$$
\begin{aligned}
V A_{n} & =-\frac{n}{2}(A, B, C, \ldots)\left(-\frac{b}{2}, 1\right)^{n-1} V b \\
& =-n A_{n-1} a^{2} .
\end{aligned}
$$

This property enables us to give a proof (exactly similar to the proof of the cognate theorem in the preceding Lecture) of the theorem that every principiant is expressible as the product of an invariant in $A_{0}, A_{1}, A_{2}, \ldots$ by a suitable power of $a$. We first observe that, using $N$ to denote $a c-b^{2}$,

$$
N, A_{0}, A_{1}, A_{2}, \ldots
$$

form a series of invariantive protomorphs of equal degree and weight.

Hence it follows that any invariant of degree $i$ and weight $w$ can be expressed in the form

$$
a^{i-w} \Phi\left(N, A_{0}, A_{1}, A_{2}, \ldots\right)
$$

and consequently that every Principiant can be expressed in this form, provided only that

$$
V \Phi=0 .
$$


Substituting for $V A_{0}, V A_{1}, V A_{2}, \ldots$ their values given above, and at the same time observing that

$$
V N=V\left(a c-b^{2}\right)=5 a^{2} b-4 a^{2} b=a^{2} b,
$$

we find $\quad V \Phi=a^{2}\left(b \partial_{N}-A_{0} \partial_{A_{1}}-2 A_{1} \partial_{A_{2}}-3 A_{2} \partial_{A_{3}}-\ldots\right) \Phi=0$.

Finally, we prove that $\Phi$ does not contain $N$, but is an invariant in $A_{0}, A_{1}, A_{2}, \ldots$ alone, by operating with $\Omega$ on

$$
\left(b \partial_{N}-A_{0} \partial_{A_{1}}-2 A_{1} \partial_{A_{2}}-3 A_{2} \partial_{A_{3}}-\ldots\right) \Phi=0,
$$

when it is easily seen that every term vanishes except the first, which gives

$$
\Omega\left(b \partial_{N} \Phi\right)=\Omega b \times \partial_{N} \Phi=0
$$

where, $\Omega b=a$ being different from zero, we must have $\partial_{N} \Phi=0$.

The invariants $N, A_{0}, A_{1}, A_{2}, \ldots$ obey a law of successive derivation similar to that which holds for the reciprocants $M, A, B, C, \ldots$

Starting with $N=a c-b^{2}$ and operating continually with

$$
G^{\prime}=a \frac{d}{d x}-(3 i+2 w) b=\left(4 a c-5 b^{2}\right) \partial_{b}+(5 a d-7 b c) \partial_{c}+\ldots
$$

we shall find

$$
\begin{aligned}
G^{\prime} N & =5 A_{0}, \\
G^{\prime} A_{0} & =6 A_{1}, \\
G^{\prime} A_{1} & =7 A_{2}-N A_{0}, \\
G^{\prime} A_{2} & =8 A_{3}-2 N A_{1}, \\
G^{\prime} A_{3} & =9 A_{4}-3 N A_{2}, \\
\cdots \cdots \cdots \cdots \cdots \cdots \cdots & \ldots \cdots \cdots \cdots \cdots \cdots \\
G^{\prime} A_{n} & =(n+6) A_{n+1}-n N A_{n-1} .
\end{aligned}
$$

and generally

These equations are exactly analogous to

$$
\begin{aligned}
& G M=5 A \\
& G A=6 B \\
& G B=7 C+M A \\
& G C=8 D+2 M B \\
& G D=9 E+3 M C
\end{aligned}
$$

in which $M=a c-\frac{5}{4} b^{2}$, and $G M, G A, G B, \ldots$ are the educts of $M, A, B, \ldots$ obtained by operating with

$$
G=a \frac{d}{d x}-(3 i+w) b=4\left(a c-b^{2}\right) \partial_{b}+5(a d-b c) \partial_{c}+\ldots
$$

It should be noticed that the two generators $G$ and $G^{\prime}$ are connected by the relation

$$
G^{\prime}=G-w b,
$$

where $w$ is the weight of the operand. 
Also, that

$$
G b=4\left(a c-b^{2}\right)=4 N, \text { and } G^{\prime} b=4 a c-5 b^{2}=4 M .
$$

We may easily verify that

$$
G^{\prime} N=5 A_{0}=5\left(a^{2} d-3 a b c+2 b^{3}\right)
$$

by operating with $G^{\prime}=\left(4 a c-5 b^{2}\right) \partial_{b}+(5 a d-7 b c) \partial_{c}$ on $N=a c-b^{2}$.

To prove that

$$
G^{\prime} A_{0}=6 A_{1} \text {, }
$$

we operate on

$$
A_{0}=A \text {, }
$$

for which the weight is 3 , with

$$
G^{\prime}=G-3 b .
$$

Thus

$$
G^{\prime} A_{0}=(G-3 b) A=6 B-3 b A=6 A_{1} .
$$

For by definition

$$
A_{1}=B-\left(\frac{b}{2}\right) A
$$

In general, to find $G^{\prime} A_{n}$, we have by definition

$$
A_{n}=(A, B, C, \ldots)\left(-\frac{b}{2}, 1\right)^{n}
$$

and, since the weight of $A_{n}$ is $n+3$,

Now

$$
G^{\prime} A_{n}=G A_{n}-(n+3) b A_{n} \text {. }
$$

$$
\begin{aligned}
G A_{n} & =G(A, B, C, \ldots)\left(-\frac{b}{2}, 1\right)^{n} \\
& =(G A, G B, G C, \ldots)\left(-\frac{b}{2}, 1\right)^{n}-\frac{n}{2}(A, B, C, \ldots)\left(-\frac{b}{2}, 1\right)^{n-1} G b .
\end{aligned}
$$

Substituting for $G A, G B, G C, \ldots$ their known values, and remembering that $G b=4 N$ and that $(A, B, C, \ldots)\left(-\frac{b}{2}, 1\right)^{n-1}=A_{n-1}$, we have

$$
\begin{aligned}
G A_{n}=(6 B, 7 C, 8 D & , \ldots)\left(-\frac{b}{2}, 1\right)^{n} \\
& +M(0, A, 2 B, 3 C, \ldots)\left(-\frac{b}{2}, 1\right)^{n}-2 n N A_{n-1} \\
= & 6(B, C, D, \ldots)\left(-\frac{b}{2}, 1\right)^{n}+(0, C, 2 D, 3 E, \ldots)\left(-\frac{b}{2}, 1\right)^{n} \\
& +M(0, A, 2 B, 3 C, \ldots)\left(-\frac{b}{2}, 1\right)^{n}-2 n N A_{n-1} .
\end{aligned}
$$

But $(0, C, 2 D, 3 E, \ldots)\left(-\frac{b}{2}, 1\right)^{n}$

$$
\begin{aligned}
& =n C\left(-\frac{b}{2}\right)^{n-1}+n(n-1) D\left(-\frac{b}{2}\right)^{n-2}+\frac{n(n-1)(n-2)}{1.2} E\left(-\frac{b}{2}\right)^{n-3}+\ldots \\
& =n(C, D, E, \ldots)\left(-\frac{b}{2}, 1\right)^{n-1}
\end{aligned}
$$


and similarly

$$
(0, A, 2 B, 3 C, \ldots)\left(-\frac{b}{2}, 1\right)^{n}=n(A, B, C, \ldots)\left(-\frac{b}{2}, 1\right)^{n-1}=n A_{n-1} .
$$

Hence

$$
G A_{n}=6(B, C, D, \ldots)\left(-\frac{b}{2}, 1\right)^{n}+n(C, D, E, \ldots)\left(-\frac{b}{2}, 1\right)^{n-1}
$$

$$
+n(M-2 N) A_{n-1} \text {. }
$$

Now let

$$
U=(A, B, C, \ldots)(u, v)^{n}
$$

then

$$
\frac{d U}{d u}=n(A, B, C, \ldots)(u, v)^{n-1}
$$

and

$$
\frac{d U}{d v}=n(B, C, D, \ldots)(u, v)^{n-1}
$$

whence it follows that

$$
\begin{aligned}
U=(A, B, C, \ldots)(u, v)^{n}=u(A, B, C, \ldots)(u, v)^{n-1} \\
+v(B, C, D, \ldots)(u, v)^{n-1}
\end{aligned}
$$

Similarly, we see that

$$
\begin{aligned}
(B, C, D, \ldots)(u, v)^{n}=u(B, C, D, \ldots) & (u, v)^{n-1} \\
& +v(C, D, E, \ldots)(u, v)^{n-1}
\end{aligned}
$$

Writing $u=-\frac{b}{2}$ and $v=1$ in the above equations, and remembering that

$$
(A, B, C, \ldots)\left(-\frac{b}{2}, 1\right)^{n}=A_{n}
$$

we obtain immediately from (1)

$$
(B, C, D, \ldots)\left(-\frac{b}{2}, 1\right)^{n-1}=A_{n}+\frac{b}{2} A_{n-1}
$$

and then (2) gives

$$
\begin{aligned}
(C, D, E, \ldots)\left(-\frac{b}{2}, 1\right)^{n-1} & =\left(A_{n+1}+\frac{b}{2} A_{n}\right)+\frac{b}{2}\left(A_{n}+\frac{b}{2} A_{n-1}\right) \\
& =A_{n+1}+b A_{n}+\frac{b^{2}}{4} A_{n-1}
\end{aligned}
$$

But it has been shown that

$$
\begin{aligned}
G A_{n}=6(B, C, D, \ldots)\left(-\frac{b}{2}, 1\right)^{n} & +n(C, D, E, \ldots)\left(-\frac{b}{2}, 1\right)^{n-1} \\
& +n(M-2 N) A_{n-1} .
\end{aligned}
$$

Hence, by substitution,

$$
\begin{aligned}
G A_{n} & =6\left(A_{n+1}+\frac{b}{2} A_{n}\right)+n\left(A_{n+1}+b A_{n}+\frac{b^{2}}{4} A_{n-1}\right)+n(M-2 N) A_{n-1} \\
& =(n+6) A_{n+1}+(n+3) b A_{n}+n\left(M+\frac{b^{2}}{4}-2 N\right) A_{n-1} .
\end{aligned}
$$


Now, $G^{\prime} A_{n}=G A_{n}-(n+3) b A_{n}=(n+6) A_{n+1}+n\left(M+\frac{b^{2}}{4}-2 N\right) A_{n-1}$,

where

$$
M+\frac{b^{2}}{4}=a c-\frac{5}{4} b^{2}+\frac{b^{2}}{4}=a c-b^{2}=N .
$$

Thus

$$
G^{\prime} A_{n}=(n+6) A_{n+1}-n N A_{n-1},
$$

which proves the law of successive derivation for the invariantive elements $A_{0}, A_{1}, A_{2}, \ldots *$.

We now proceed to explain the method of transforming a Principiant, given in terms of the small letters $a, b, c, \ldots$, into one expressed in terms of $a, A, B, C, \ldots$.

Remembering that the expressions for

$$
A, B, C, D, E, \ldots
$$

have for their most advanced small letters

$$
d, e, f, g, h, \ldots,
$$

and that, in each capital letter, the most advanced letter occurs only in the first degree, multiplied by a power of $a$, it follows, as an immediate consequence, that we may, by continually substituting for the most advanced letter, eliminate $d, e, f, g, h, \ldots$ from any rational integral function

$$
\phi(a, b, c, d, e, f, g, h, \ldots)
$$

and thus transform it into another function whose arguments are

$$
a, b, c, A, B, C, D, E, \ldots
$$

and which is rational in all its arguments, and integral in all of them, with the possible exception of the first argument, $a$.

But (see Lecture XXVIII.) [above, p. 471] the result of this elimination is known to be

$$
a^{i-w} \Phi(A, B, C, D, E, \ldots)
$$

in the case where $\phi$ is a Principiant of known degree $i$ and weight $w$. Hence $b$ and $c$ must disappear spontaneously during the process of elimination.

This being so, we can give $b$ and $c$ any arbitrary values, without thereby affecting the result, and it will greatly simplify the work to take $b=0$ and $c=0$.

It is also permissible to take $a=1$; for, although the factor $a^{i-w}$ is thereby lost, it can always be restored in the final result because both $i$ and

\footnotetext{
* The establishment of the seale of relation between the terms of the $A_{0}, A_{1}, A_{2}, \ldots$ series, and the above proof of it, is due exclusively to Mr Hammond.
} 
$w$ are known numbers. Now, if we write $a=1, b=0, c=0$ in the known expressions for $A, B, C, D, \ldots$, we shall find

$$
\begin{aligned}
& A=d \\
& B=e \\
& C=f \\
& D=g-\frac{25}{8} d^{2} \\
& E=h-\frac{15}{2} d e,
\end{aligned}
$$

Hence we have to eliminate $d, e, f, g, h, \ldots$ between the above equations and

$$
P=\phi(1,0,0, d, e, f, g, h, \ldots),
$$

where $P$ stands for the given Principiant. In other words, we have to substitute for

$$
\begin{array}{lllllllll}
a, & b, & c, & d, & e, & f, & g, & h, & \ldots \\
1, & 0, & 0, & A, & B, & C, & D+\frac{25}{8} A^{2}, & E+\frac{15}{2} A B, \ldots \\
& & P=\phi(a, b, c, d, e, f, g, h, \ldots) .
\end{array}
$$

The result of this substitution will be

$$
P=\Phi(A, B, C, D, E, \ldots),
$$

where, to compensate for the factor lost by taking $a=1$, we must multiply $\Phi$ by $a^{i-w}$. As an easy example, consider the Principiant which Halphen calls $\Delta$, and for which he obtains the expression

$$
\left|\begin{array}{cccll}
b & c & d & e & f \\
a & b & c & d & e \\
-a^{2} & 0 & b^{2} & 2 b c & 2 b d+c^{2} \\
0 & a^{2} & 2 a b & 2 a c+b^{2} & 2 a d+2 b c \\
0 & 0 & a^{2} & 3 a b & 3 b^{2}+3 a c
\end{array}\right|
$$

Here the degree $i=8$ and the weight $w=8$; so that $i-w=0$, and no factor has to be restored. On making the substitutions spoken of, the determinant becomes

$$
\left|\begin{array}{rrrrc}
0 & 0 & A & B & C \\
1 & 0 & 0 & A & B \\
-1 & 0 & 0 & 0 & 0 \\
0 & 1 & 0 & 0 & 2 A \\
0 & 0 & 1 & 0 & 0
\end{array}\right|
$$

which immediately reduces to $A C-B^{2}$ by striking out the first three columns and the last three rows.

Of this Principiant we shall have more to say hereafter. 


\section{LECTURE XXX.}

The method of substituting large letters for small ones will be better understood if we employ it to obtain an expression of the form

$$
a^{i-w} \Phi(M, A, B, C, D, E, \ldots)
$$

for any pure reciprocant

$$
\phi(a, b, c, d, e, f, g, h, \ldots)
$$

of known degree $i$ and weight $w$ in the small letters.

The transformation is effected by substituting in $\phi$ for $c, d, e, f, g, h, \ldots$ their values (which are perfectly definite) in terms of $a, b, M, A, B, C, D, E, \ldots$ But since $b$ does not appear in the final result, we are at liberty to give it any arbitrary value, and it will be convenient to take $b=0$, for then (see Lecture XXVIII.) [above, p. 465] we have

$$
\begin{aligned}
& M=a c \\
& A=a^{2} d \\
& B=a^{3} e-2 a^{2} c^{2} \\
& C=a^{4} f-5 a^{3} c d \\
& D=a^{5} g-\frac{25}{8} a^{4} d^{2}-6 a^{4} c e+7 a^{3} c^{3} \\
& E=a^{6} h-\frac{15}{2} a^{5} d e-7 a^{5} c f+29 a^{4} c^{2} d
\end{aligned}
$$

There is an additional advantage in taking $b=0$, namely, that then the values of the invariants $N, A_{0}, A_{1}, A_{2}, \ldots$ (see their definition at the beginning of * Lecture XXIX.) exactly coincide with those of the reciprocants $M, A, B, C, \ldots$ set forth above. Hence, merely interchanging the capital letters, the same substitutions enable us to express any invariant in terms of $a, N, A_{0}, A_{1}, \ldots$, as well as any reciprocant in terms of $a, M, A, B, \ldots$

The solution of the above equations will give $\frac{c}{a}, \frac{d}{a}, \frac{e}{a}, \ldots$ in terms of $\frac{M}{a^{2}}, \frac{A}{a^{3}}, \frac{B}{a^{4}}, \ldots ;$ but we can, without loss of generality, put $a=1$, when we shall find

$$
\begin{aligned}
a & =1, \\
b & =0, \\
c & =M, \\
d & =A \\
e & =B+2 M^{2}, \\
f & =C+5 M A \\
g & =D+\frac{25}{8} A^{2}+6 M B+5 M^{3}, \\
h & =E+\frac{15}{2} A B+7 M C+6 M A^{2},
\end{aligned}
$$

[* p. 472, above.] 
The substitution of these values in the pure reciprocant

will convert it into

$$
\phi(a, b, c, d, e, f, g, h, \ldots)
$$$$
\Phi(M, A, B, C, D, E, \ldots) \text {. }
$$

We have written $a=1$ for the sake of simplicity; but without doing this we have, since $\phi$ is homogeneous of degree $i$,

$$
\phi(a, 0, c, d, e, \ldots)=a^{i} \phi\left(1,0, \frac{c}{a}, \frac{d}{a}, \frac{e}{a}, \ldots\right) .
$$

Hence, substituting for $\frac{c}{a}, \frac{d}{a}, \frac{e}{a}, \ldots$ in terms of $\frac{M}{a^{2}}, \frac{A}{a^{3}}, \frac{B}{a^{4}}, \ldots$,

$$
\phi(a, 0, c, d, e, \ldots)=a^{i} \Phi\left(\frac{M}{a^{2}}, \frac{A}{a^{3}}, \frac{B}{a^{4}}, \ldots\right) ;
$$

or, since $M, A, B, \ldots$ are of weights $2,3,4, \ldots$ and $\Phi$ is of weight $w$,

$$
\phi(a, 0, c, d, e, \ldots)=a^{i-w} \Phi(M, A, B, \ldots) .
$$

Thus, in consequence of writing $a=1$, the factor $a^{i-w}$ has been lost; but this factor can always be restored, both $i$ and $w$ being known numbers.

When $\phi$ is a Principiant, $M$ will not appear in the final result, which will be identical with that obtained by the simpler substitutions of the preceding Lecture. If, for example, we substitute for

$$
\begin{array}{cccccc}
a, & b, & c, & d, & e, & f, \\
1, & 0, & M, & A, & B+2 M^{2}, & C+5 M A, \\
1, & 0, & 0, & A, & B, & C,
\end{array}
$$

instead of

in the determinant expression for Halphen's $\Delta$, previously given, it becomes

$$
\left|\begin{array}{rcccc}
0 & M & A & B+2 M^{2} & C+5 M A \\
1 & 0 & M & A & B+2 M^{2} \\
-1 & 0 & 0 & 0 & M^{2} \\
0 & 1 & 0 & 2 M & 2 A \\
0 & 0 & 1 & 0 & 3 M
\end{array}\right| .
$$

Subtracting the 4th row multiplied by $M$ from the first, the determinant reduces to

$$
\left|\begin{array}{rccc}
0 & A & B & C+3 M A \\
1 & M & A & B+2 M^{2} \\
-1 & 0 & 0 & M^{2} \\
0 & 1 & 0 & 3 M
\end{array}\right|
$$

Again, subtracting the 2nd column multiplied by $3 M$ from the last, and reducing, the determinant becomes

$$
\left|\begin{array}{rcc}
0, & B, & C \\
1, & A, & B-M^{2} \\
-1, & 0, & M^{2}
\end{array}\right|=A C-B^{2}
$$

where $M$ disappears, as it ought to do, because $\Delta$ is a Principiant. 
In what follows we shall have frequent occasion to make use of the fact that if $R_{a}$ is an absolute pure reciprocant, $\frac{d R_{a}}{a^{\frac{1}{3}} d x}$, which we know is a pure reciprocant, is also an absolute one.

This is very easily proved. For let $R$ be any pure reciprocant, of degree $i$ and weight $w$, which becomes $R_{a}$ when made absolute by division by a power of $a$, then

$$
R_{a}=\frac{R}{a^{\frac{\mu}{3}}}, \text { where } \mu=3 i+w,
$$

and, using $G$ as usual to denote the generator for pure reciprocants,

$$
\begin{gathered}
\frac{d R_{a}}{d x}=\frac{G R}{a^{\frac{\mu}{3}+1}} . \\
\frac{d R_{a}}{a^{\frac{1}{3}} d x}=\frac{G R}{a^{\frac{\mu+4}{3}}},
\end{gathered}
$$

Hence

which is an absolute pure reciprocant because $G R$, which is of degree $i+1$ and weight $w+1$, must be divided by $a^{\frac{\mu+4}{3}}$ in order to make it absolute. Thus, if $M_{a}, A_{a}, B_{a}, C_{a}, \ldots$ are what $M, A, B, C, \ldots$ become when each of them is made absolute by division by a power of $a$, we have

$$
\begin{aligned}
& a^{-\frac{1}{3}} \frac{d}{d x} M_{a}=5 A_{a} \\
& a^{-\frac{1}{3}} \frac{d}{d x} A_{a}=6 B_{a} \\
& a^{-\frac{1}{3}} \frac{d}{d x} B_{a}=7 C_{a}+M_{a} A_{a}
\end{aligned}
$$

We shall use these results in deducing the complete primitive of the differential equation

$$
A C-B^{2}=0
$$

from that of the equation in pure reciprocants,

$$
25 A^{2}-16 M^{3}=0
$$

This equation may be written in the form

$$
25 A_{a}{ }^{2}=16 M_{a}^{3} ;
$$

whence, by differentiation, we obtain

$$
50 A_{a}\left(a^{-\frac{1}{3}} \frac{d}{d x} A_{a}\right)=48 M_{a}^{2}\left(a^{-\frac{1}{3}} \frac{d}{d x} M_{a}\right),
$$

which gives

$$
50 A_{a} .6 B_{a}=48 M_{a}^{2} .5 A_{a} ;
$$

that is,

$$
5 B_{a}=4 M_{a}{ }^{2} \text {. }
$$


Differentiating this result, we find

$$
5\left(7 C_{a}+M_{a} A_{a}\right)=40 M_{a} A_{a} ;
$$

which gives

$$
C_{a}=M_{a} A_{a} \text {. }
$$

We now restore the non-absolute reciprocants $M, A, B, C$; that is, we write

$$
5 B=4 M^{2} \text { and } C=M A .
$$

Hence $25\left(A C-B^{2}\right)=M\left(25 A^{2}-16 M^{3}\right)=0$ (because $\left.25 A^{2}=16 M^{3}\right)$.

Now, the equation $A C-B^{2}=0$ remains unaltered by any homographic substitution, so that it will be satisfied not only by any solution of the equation in pure reciprocants $25 A^{2}-16 M^{3}=0$, but also by any homographic transformation of such solution. But it has been shown (in Lecture XIII., [p. 379, above]) that the complete primitive of $25 A^{2}-16 M^{3}=0$ is a linear transformation of $y=x^{\lambda}$, where $\lambda^{2}-\lambda+1=0$ (that is, where $\lambda$ is a cube root of negative unity).

Consequently any homographic transformation of $y=x^{\lambda}$ is a solution of

$$
A C-B^{2}=0 \text {. }
$$

Moreover, this is its complete primitive; for the highest letter, $f$, which occurs in $A C-B^{2}$, corresponds to the seventh order of differentiation, and if we write

$$
y=\frac{Y}{Z}, \quad x=\frac{X}{Z}
$$

where $X, Y, Z$ are general linear functions of $x, y, 1$ (that is, if we make the most general homographic substitution), $y=x^{\lambda}$ becomes $Y=X^{\lambda} Z^{1-\lambda}$, which will be found to contain exactly 7 independent arbitrary constants. Thus the complete primitive of $A C-B^{2}=0$ is $Y=X^{\lambda} Z^{1-\lambda}$, where $X, Y, Z$ are general linear functions of $x, y, 1$, and $\lambda$ is a cube root of negative unity.

Observe that although any solution of $M=0$ also makes $A, B, C, \ldots$ all vanish, and so satisfies $A C-B^{2}=0$, we cannot from this infer that a homographic transformation of the parabola $y=x^{2}$ will be the complete primitive of $A C-B^{2}=0$. For, though $Y Z=X^{2}$ is a solution of $A C-B^{2}=0$, it only contains 5 independent arbitrary constants, and therefore cannot be its complete primitive. Neither can $Y Z=X^{2}$ be obtained from the complete primitive by giving special values to the arbitrary constants. Hence $Y Z=X^{2}$ is a singular solution of $A C-B^{2}=0$.

We may also deduce the differential equation of the curve $Y=X^{\lambda} Z^{1-\lambda}$, where $\lambda$ has a general value, from the corresponding equation in pure reciprocants,

$$
25\left(2 \lambda^{2}-5 \lambda+2\right) A^{2}+16(\lambda+1)^{2} M^{3}=0,
$$

which has (see [p. 377, above]) for its complete primitive any linear transformation of the general parabola $y=x^{\lambda}$. 
Writing for shortness

$$
2 \lambda^{2}-5 \lambda+2=p \text { and }(\lambda+1)^{2}=q,
$$

and at the same time making both $A$ and $M$ absolute, the above equation becomes

$$
25 p A_{a}{ }^{2}+16 q M_{a}{ }^{3}=0 .
$$

Hence, by differentiation, we obtain

which gives

$$
50 p A_{a} .6 B_{a}+48 q M_{a}^{2} .5 A_{a}=0,
$$

$$
5 p B_{a}+4 q M_{a}^{2}=0 .
$$

After a second differentiation we find

$$
5 p\left(7 C_{a}+M_{a} A_{a}\right)+40 q M_{a} A_{a}=0 ;
$$

that is,

$$
7 p C_{a}+(p+8 q) M_{a} A_{a}=0 .
$$

We now replace the absolute reciprocants $M_{a}, A_{a}, B_{a}, C_{a}$ by $M, A, B, C$, and thus write the original equation and its two differentials in the form

Hence we find

$$
\begin{aligned}
25 p A^{2} & =-16 q M^{3}, \\
5 p B & =-4 q M^{2}, \\
7 p C & =-(p+8 q) M A .
\end{aligned}
$$

$$
\begin{aligned}
5^{2} \cdot 7 \cdot p^{2}\left(A C-B^{2}\right) & =-25 p(p+8 q) M A^{2}-16.7 q^{2} M^{4} \\
& =16 q(p+q) M^{4} \\
5^{6} \cdot 7^{3} \cdot p^{6}\left(A C-B^{2}\right)^{3} & =16^{3} q^{3}(p+q)^{3} M^{12} \\
5^{8} p^{4} A^{8} & =16^{4} q^{4} M^{12}
\end{aligned}
$$

and, eliminating $M$ from the two last equations,

$$
2^{4} \cdot 7^{3} \cdot p^{2} q\left(A C-B^{2}\right)^{3}=5^{2}(p+q)^{3} A^{8} .
$$

Now restoring $\quad p=2 \lambda^{2}-5 \lambda+2=(\lambda-2)(2 \lambda-1)$

and

$$
q=(\lambda+1)^{2}
$$

we have

$$
p+q=3\left(\lambda^{2}-\lambda+1\right)
$$

so that the final equation becomes

$$
2^{4} \cdot 7^{3}(\lambda+1)^{2}(\lambda-2)^{2}(2 \lambda-1)^{2}\left(A C-B^{2}\right)^{3}=3^{3} .5^{2}\left(\lambda^{2}-\lambda+1\right)^{3} A^{8} .
$$

The same reasoning as before will show that, for a general value of $\lambda$, the complete primitive of this equation is the general homographic transformation $Y=X^{\lambda} Z^{1-\lambda}$ of the curve $y=x^{\lambda}$.

There is, however, a special exceptional case in which the differential equation becomes

$$
2^{6} \cdot 7^{3}\left(A C-B^{2}\right)^{3}=3^{3} \cdot 5^{2} A^{8},
$$

the corresponding value of the parameter $\lambda$ being either 0,1 or $\infty$, as may be seen by solving the equation

$$
(\lambda+1)^{2}(\lambda-2)^{2}(2 \lambda-1)^{2}=4\left(\lambda^{2}-\lambda+1\right)^{3} .
$$


In the case where $\lambda=0$ or $\infty$ we can, in the same manner as before, show that the complete primitive is a homographic transformation of the curve $y=e^{x}$ by deducing the differential equation from the corresponding equation in pure reciprocants,

$$
25 A^{2}+8 M^{3}=0
$$

whose complete primitive is (see Lecture XIII.) [p. 379 above] a linear transformation of $y=e^{x}$.

When $\lambda=1$ the corresponding equation in pure reciprocants is

$$
25 A^{2}-64 M^{3}=0
$$

whose complete primitive may be shown to be a linear transformation of $y=x \log x$. The reason why these two distinct equations in pure reciprocants lead to the same equation in principiants is that the two curves $y=e^{x}$ and $y=x \log x$ are homographically equivalent but not linearly transformable into one another. For we may write the equation $y=x \log x$ in the form $x=e^{\frac{y}{x}}$, which is a homographic transformation of $y=e^{x}$.

Besides the special case just considered, in which the complete primitive of the equation in Principiants is $\frac{Y}{Z}=e^{\frac{X}{Z}}$, we may notice that in which the parameter $\lambda$ is either $-1,2$, or $\frac{1}{2}$, the differential equation reducing to $A=0$ simply, and its complete primitive $Y=X^{\lambda} Z^{1-\lambda}$ being the equation to a conic, as it should be. The case where $\lambda^{2}-\lambda+1=0$ and the differential equation reduces to $A C-B^{2}=0$ has been considered already. There remains the case in which $\lambda=3$, when the complete primitive becomes $Y Z^{2}=X^{3}$ (the equation of the general cuspidal cubic) and the differential equation assumes the simple form

$$
\left(\frac{A C-B^{2}}{3}\right)^{3}=\left(\frac{A}{2}\right)^{8}
$$

which is therefore the differential equation of cuspidal cubics.

We shall hereafter show that in this case the Principiant

$$
2^{8}\left(A C-B^{2}\right)^{3}-3^{3} A^{8}
$$

which is apparently of the 24th degree, loses a factor $a^{4}$ and so sinks to the 20th degree. It is, however, generally difficult to determine the power of $a$ contained as a factor in a Principiant given in terms of the large letters.

The results obtained in the present Lecture agree with those of M. Halphen contained in his Thèse sur les Invariants différentiels (Paris, Gauthier-Villars, 1878), which contains a complete investigation of the properties of the Principiant $A C-B^{2}$, which he calls $\Delta$. But our point of 
view is different from his. He obtains $\Delta$ in the form of a determinant from geometrical considerations. With him $\Delta=0$ is the differential equation which expresses the condition that, at a point $x, y$ on any curve, a nodal cubic shall exist, having its node at $x, y$, and such that one of its branches shall have 8-point contact with the curve at that point. With us $A C-B^{2}$ is the simplest example, after the Mongian $A$, of an invariant in the capital letters $A, B, C, \ldots$.

\section{LECTURE XXXI.}

We may include $\lambda$ among the arbitrary constants in the primitive equation $Y=X^{\lambda} Z^{1-\lambda}$, which can also be written in the form

$$
\lambda \log X-\log Y+(1-\lambda) \log Z=0,
$$

or $(X, Y, Z$ being general linear functions of $x, y, 1)$ in the equivalent form

$\lambda \log (y+\alpha x+\beta)-\log \left(y+\alpha^{\prime} x+\beta^{\prime}\right)+(1-\lambda) \log \left(y+\alpha^{\prime \prime} x+\beta^{\prime \prime}\right)=$ const. which evidently contains 8 independent arbitrary constants.

One of these will be made to disappear by differentiation, and thus we shall obtain a differential equation of the first order, containing 7 arbitrary constants, identical (when the constants are rearranged) with

$$
(y-x t)(l x+m y)+t\left(l^{\prime} x+m^{\prime} y+n^{\prime}\right)+l^{\prime \prime} x+m^{\prime \prime} y+n^{\prime \prime}=0,
$$

which is known as Jacobi's Equation.

For, by differentiating the primitive equation, we obtain

$$
\begin{aligned}
\lambda(t+\alpha)(y+\alpha x+\beta)^{-1}-\left(t+\alpha^{\prime}\right) & \left(y+\alpha^{\prime} x+\beta^{\prime}\right)^{-1} \\
& +(1-\lambda)\left(t+\alpha^{\prime \prime}\right)\left(y+\alpha^{\prime \prime} x+\beta^{\prime \prime}\right)^{-1}=0,
\end{aligned}
$$

which, when cleared of negative indices by multiplication, becomes

$$
\begin{aligned}
& \lambda\left(y+\alpha^{\prime} x+\beta^{\prime}\right)\left\{\left(y+\alpha^{\prime \prime} x+\beta^{\prime \prime}\right)(t+\alpha)-(y+\alpha x+\beta)\left(t+\alpha^{\prime \prime}\right)\right\} \\
& \quad+(y+\alpha x+\beta)\left\{\left(y+\alpha^{\prime} x+\beta^{\prime}\right)\left(t+\alpha^{\prime \prime}\right)-\left(y+\alpha^{\prime \prime} x+\beta^{\prime \prime}\right)\left(t+\alpha^{\prime}\right)\right\}=0 .
\end{aligned}
$$

Writing this equation in the equivalent form

$$
\begin{aligned}
& \lambda\left(y+\alpha^{\prime} x+\beta^{\prime}\right)\left\{\left(\alpha-\alpha^{\prime \prime}\right)(y-x t)+\left(\beta^{\prime \prime}-\beta\right) t+\left(\alpha \beta^{\prime \prime}-\alpha^{\prime \prime} \beta\right)\right\} \\
& \quad+(y+\alpha x+\beta)\left\{\left(\alpha^{\prime \prime}-\alpha^{\prime}\right)(y-x t)+\left(\beta^{\prime}-\beta^{\prime \prime}\right) t+\left(\alpha^{\prime \prime} \beta^{\prime}-\alpha^{\prime} \beta^{\prime \prime}\right)\right\}=0,
\end{aligned}
$$

it is easily seen to be identical with Jacobi's equation given above.

The seven arbitrary constants which occur in Jacobi's equation are the mutual ratios of the eight coefficients $l, m, l^{\prime}, m^{\prime}, n^{\prime}, l^{\prime \prime}, m^{\prime \prime}, n^{\prime \prime}$, any one of which may have an arbitrarily chosen value assigned to it.

Taking $m=-1$, the equation may be written in the form

where

$$
\begin{gathered}
P t+l x y-y^{2}+l^{\prime \prime} x+m^{\prime \prime} y+n^{\prime \prime}=0, \\
P=l^{\prime} x+m^{\prime} y+n^{\prime}-l x^{2}+x y .
\end{gathered}
$$


In order to eliminate $n^{\prime \prime}$ and $l^{\prime \prime}$, we differentiate the above equation twice. The first differentiation gives

$$
2 a P+t\left(P^{\prime}+l x-2 y+m^{\prime \prime}\right)+l y+l^{\prime \prime}=0,
$$

where $P^{\prime}=\frac{d P}{d x}=l^{\prime}+m^{\prime} t-2 l x+y+x t$, and the second differentiation gives

$$
6 b P+2 a\left(2 P^{\prime}+l x-2 y+m^{\prime \prime}\right)+t\left(P^{\prime \prime}+2 l-2 t\right)=0 .
$$

Now, $P^{\prime \prime}=\frac{d P^{\prime}}{d x}=2 a\left(m^{\prime}+x\right)+2(t-l) ;$ so that, on substituting this value, the above equation becomes

where

$$
3 b P+a Q=0
$$

$$
\begin{aligned}
Q & =2 P^{\prime}+l x-2 y+m^{\prime \prime}+m^{\prime} t+x t \\
& =2 l^{\prime}+3 m^{\prime} t-3 l x+3 x t+m^{\prime \prime} .
\end{aligned}
$$

Differentiating (1) we have

$$
12 c P+3 b P^{\prime}+3 b Q+a Q^{\prime}=0
$$

where

$$
Q^{\prime}=3(t-l)+6 a\left(x+m^{\prime}\right)=3 R+6 a S, \text { suppose. }
$$

Thus we have $\quad 4 c P+b P^{\prime}+b Q+a R+2 a^{2} S=0$.

Differentiating this 4 times in succession, and at each step substituting for

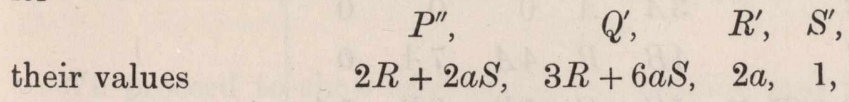

we obtain 4 more equations, from which, combined with the 2 previously obtained, we can eliminate

$$
P, P^{\prime}, Q, R, S
$$

Thus, differentiating (2), we find

$$
\begin{aligned}
20 d P+8 c P^{\prime}+b(2 R+2 a S) & +4 c Q+b(3 R+6 a S) \\
& +3 b R+2 a^{2}+12 a b S+2 a^{2}=0 \\
5 &
\end{aligned}
$$

that is,

and continuing the same process,

$$
\begin{aligned}
& 6 e P+3 d P^{\prime}+d Q+3 c R+\left(6 a c+3 b^{2}\right) S+3 a b=0 \\
& 7 f P+4 e P^{\prime}+e Q+4 d R+(7 a d+7 b c) S+\left(4 a c+2 b^{2}\right)=0, \\
& 8 g P+5 f P^{\prime}+f Q+5 e R+\left(8 a e+8 b d+4 c^{2}\right) S+(5 a d+5 b c)=0 .
\end{aligned}
$$

The result of elimination is

$$
\left|\begin{array}{llllll}
3 b & 0 & a & 0 & 0 & 0 \\
4 c & b & b & a & 2 a^{2} & 0 \\
5 d & 2 c & c & 2 b & 5 a b & a^{2} \\
6 e & 3 d & d & 3 c & 6 a c+3 b^{2} & 3 a b \\
7 f & 4 e & e & 4 d & 7 a d+7 b c & 4 a c+2 b^{2} \\
8 g & 5 f & f & 5 e & 8 a e+8 b d+4 c^{2} & 5 a d+5 b c
\end{array}\right|=0
$$

where the determinant equated to zero is a Principiant. 
In his Thèse sur les Invariants différentiels, p. 42, M. Halphen states that this equation can be found by eliminating the constants from Jacobi's equation, but he does not set out the work. When in the above determinant twice the 3rd column is added to the second, it becomes exactly identical with the one given by Halphen, which he calls $T$.

We proceed to express the above result in terms of the capital letters, using the method explained in Lecture XXIX., and observing that the determinant is of degree 8 and of weight 12 ; so that in this case $i-w=8-12=-4$, showing that the final result has to be multiplied by $a^{-4}$.

Substituting in the determinant for

$$
\begin{array}{lllllll}
a & b & c & d & e & f & g \\
1 & 0 & 0 & A & B & C & D+\frac{25}{8} A^{2}
\end{array}
$$

it becomes

$\left|\begin{array}{llllll}0 & 0 & 1 & 0 & 0 & 0 \\ 0 & 0 & 0 & 1 & 2 & 0 \\ 5 A & 0 & 0 & 0 & 0 & 1 \\ 6 B & 3 A & A & 0 & 0 & 0 \\ 7 C & 4 B & B & 4 A & 7 A & 0 \\ 8 D+25 A^{2} & 5 C & C & 5 B & 8 B & 5 A\end{array}\right|$

Subtracting the last column multiplied by $5 \mathrm{~A}$ from the first, and the 4 th column multiplied by 2 from the 5 th, and then striking out rows and columns, we obtain

$$
\begin{aligned}
& \left|\begin{array}{llllcl}
0 & 0 & 1 & 0 & 0 & 0 \\
0 & 0 & 0 & 1 & 0 & 0 \\
0 & 0 & 0 & 0 & 0 & 1 \\
6 B & 3 A & A & 0 & 0 & 0 \\
7 C & 4 B & B & 4 A & -A & 0 \\
8 D & 5 C & C & 5 B & -2 B & 5 A
\end{array}\right| \\
& =\left|\begin{array}{lllcl}
0 & 0 & 1 & 0 & 0 \\
0 & 0 & 0 & 0 & 1 \\
6 B & 3 A & 0 & 0 & 0 \\
7 C & 4 B & 4 A & -A & 0 \\
8 D & 5 C & 5 B & -2 B & 5 A
\end{array}\right| \\
& =\left|\begin{array}{llll}
0 & 0 & 0 & 1 \\
6 B & 3 A & 0 & 0 \\
7 C & 4 B & -A & 0 \\
8 D & 5 C & -2 B & 5 A
\end{array}\right|=\mid \begin{array}{lll}
6 B & 3 A & 0 \\
7 C & 4 B & A \\
8 D & 5 C & 2 B
\end{array} \\
& =24\left(A^{2} D-3 A B C+2 B^{3}\right) \text {. }
\end{aligned}
$$


If, using Halphen's notation, we call the principiant now under consideration $T$, what we have proved is that

$$
T=24 a^{-4}\left(A^{2} D-3 A B C+2 B^{3}\right)
$$

and consequently that $A^{2} D-3 A B C+2 B^{3}$ is divisible by $a^{4}$.

The differential equation $T=0$ corresponds, as we have seen, to the complete primitive $Y=X^{\lambda} Z^{1-\lambda}$, in which $\lambda$ is counted as one of the arbitrary constants.

This result may be otherwise obtained. For we have shown in the preceding Lecture that the differential equation of the seventh order, from which all the arbitrary constants except $\lambda$ have disappeared, has the form

where $\kappa$ depends solely on $\lambda$.

$$
\left(A C-B^{2}\right)^{3}=\kappa A^{8}
$$

Writing this equation in the form

$$
\left(A C-B^{2}\right) A^{-\frac{8}{3}}=\text { const., }
$$

and differentiating with respect to $x$, we remove the remaining arbitrary constant, and thus obtain the differential equation of the 8th order free from all arbitrary constants, a result which, to a factor près, must coincide with

$$
T=0 \text {. }
$$

We proceed to show how this differentiation may be performed without introducing any of the small letters. In the first place, it is clear that since

$$
G=4\left(a c-b^{2}\right) \partial_{b}+5(a d-b c) \partial_{c}+6(a e-b d) \partial_{d}+\ldots
$$

does not contain $\partial_{a}$ and is linear in the other differential reciprocals $\partial_{b}, \partial_{c}, \ldots$,

$$
\begin{aligned}
G a^{\theta} \Phi(A, B, C, \ldots) & =a^{\theta} G \Phi(A, B, C, \ldots) \\
& =a^{\theta}\left(\frac{d \Phi}{d A} G A+\frac{d \Phi}{d B} G B+\frac{d \Phi}{d C} G C+\ldots\right)
\end{aligned}
$$

And since we have

$$
\begin{aligned}
& G A=6 B \\
& G B=7 C+M A \\
& G C=8 D+2 M B
\end{aligned}
$$

it follows immediately that

$$
\begin{aligned}
G a^{\theta} \Phi(A, B, C, \ldots) & =a^{\theta}\left(6 B \partial_{A}+7 C \partial_{B}+8 D \partial_{C}+\ldots\right) \Phi \\
& +a^{\theta} M\left(A \partial_{B}+2 B \partial_{C}+3 C \partial_{D}+\ldots\right) \Phi
\end{aligned}
$$

This is true for any function of the capital letters, whatever its nature may be; but when $\Phi$ is a principiant, it is also an invariant in the large letters; so that in this case we have

and

$$
\begin{gathered}
\left(A \partial_{B}+2 B \partial_{C}+3 C \partial_{D}+\ldots\right) \Phi=0 \\
G a^{\theta} \Phi=a^{\theta}\left(6 B \partial_{A}+7 C \partial_{B}+8 D \partial_{C}+\ldots\right) \Phi
\end{gathered}
$$


Now, the operation of $G$ on a function of degree $i$ and weight $w$ is equivalent to that of $a \frac{d}{d x}-(3 i+w) b$, or to that of $a \frac{d}{d x}$, when both $i=0$ and $w=0$ (which happens in the case of a plenarily absolute form). Hence, if we suppose $\Phi$ to be a plenarily absolute principiant, $G \Phi$ is also a principiant, though not a plenarily absolute one.

For $a$ is a principiant, and $\frac{d \Phi}{d x}$ is a principiant; therefore $a \frac{d \Phi}{d x}$ or $G \Phi$ is one also*. Thus

$$
6 B \partial_{A}+7 C \partial_{B}+8 D \partial_{C}+\ldots
$$

acting on any plenarily absolute principiant, generates another principiant, but not a plenarily absolute one.

We now resume the consideration of the equation

$$
\left(A C-B^{2}\right) A^{-\frac{8}{3}}=\text { const. }
$$

Differentiating and multiplying by $a$, we have

$$
a \frac{d}{d x}\left\{\left(A C-B^{2}\right) A^{-\frac{8}{3}}\right\}=0
$$

Hence, by what precedes,

$$
\left(6 B \partial_{A}+7 C \partial_{B}+8 D \partial_{C}\right)\left\{\left(A C-B^{2}\right) A^{-\frac{8}{3}}\right\}=0 ;
$$

or, using $\Theta$ to denote the operator,

$$
\begin{gathered}
6 B \partial_{A}+7 C \partial_{B}+8 D \partial_{C}+\ldots \\
A^{-\frac{8}{3}} \Theta\left(A C-B^{2}\right)-\frac{8}{3} A^{-\frac{11}{3}}\left(A C-B^{2}\right) \Theta A=0
\end{gathered}
$$

or, observing that $\Theta A=6 B$,

$$
A \Theta\left(A C-B^{2}\right)-16 B\left(A C-B^{2}\right)=0 .
$$

This gives $A(6 B C-14 B C+8 A D)-16 B\left(A C-B^{2}\right)=0$; or finally

$$
A^{2} D-3 A B C+2 B^{3}=0 \text {. }
$$

We may find a generator for principiants expressed in terms of the large letters similar to the expression for the reciprocant generator $G$ in terms of

* See the concluding paragraph of Lecture XXV. [p. 450 above], where it was shown that $P$, being a principiant (of degree $i$ and weight $w$ ), $a \frac{d P}{d x}-(3 i+w) b P$ is a reciprocant, and $a \frac{d P}{d x}-(3 i+2 w) b P$ an invariant. This proves, what we omitted to mention there, that $P$ being a zero-weight principiant,

$$
G P=\left(a \frac{d}{d x}-3 i b\right) P \text { is a principiant. }
$$

It may here be remarked that a principiant of degree $i$ and of zero weight is equal to the corresponding plenarily absolute principiant (which is a function of the large letters only) multiplied by the factor $a^{i}$, on which the operator $G$ does not act. 
the small letters. For let $P$ be any principiant, of weight $w$, which, when reduced to zero weight by division by $A^{\frac{w}{3}}$, becomes $P A^{-\frac{w}{3}}$; then

is a principiant. But

$$
\Theta\left(P A^{-\frac{w}{3}}\right)
$$

$$
\Theta\left(P A^{-\frac{w}{3}}\right)=A^{-\frac{w}{3}-1}(A \Theta-2 w B) P,
$$

where, remembering that $A^{=\frac{w}{3}-1}$ is a principiant, $(A \Theta-2 w B) P$ is one also.

Now, the weights of

$$
A, B, C, D, \ldots
$$

being

$3,4,5,6, \ldots$,

we may write

$$
w=3 A \partial_{A}+4 B \partial_{B}+5 C \partial_{C}+6 D \partial_{D}+\ldots,
$$

and consequently

$$
\begin{aligned}
A \Theta-2 w B= & A\left(6 B \partial_{A}+7 C \partial_{B}+8 D \partial_{C}+9 E \partial_{D}+\ldots\right) \\
& -2 B\left(3 A \partial_{A}+4 B \partial_{B}+5 C \partial_{C}+6 D \partial_{D}+\ldots\right) \\
= & \left(7 A C-8 B^{2}\right) \partial_{B}+(8 A D-10 B C) \partial_{C}+(9 A E-12 B D) \partial_{D}+\ldots,
\end{aligned}
$$

which is the generator in question.

As an easy example of its use, suppose it to operate on $A C-B^{2}$; then

$$
\begin{aligned}
& \left\{\left(7 A C-8 B^{2}\right) \partial_{B}+(8 A D-10 B C) \partial_{C}\right\}\left(A C-B^{2}\right) \\
& =-2 B\left(7 A C-8 B^{2}\right)+A(8 A D-10 B C) \\
& =8\left(A^{2} D-3 A B C+2 B^{3}\right) .
\end{aligned}
$$

The generator just obtained,

$$
\left(7 A C-8 B^{2}\right) \partial_{B}+(8 A D-10 B C) \partial_{C}+(9 A E-12 B D) \partial_{D}+\ldots,
$$

is a linear combination of Cayley's two generators (given in Lecture IV., [p. 327, above]), which, when we write $A, B, C, \ldots$ instead of the corresponding small letters, become

$$
\left(A C-B^{2}\right) \partial_{B}+(A D-B C) \partial_{C}+(A E-B D) \partial_{D}+\ldots
$$

and

$$
\left(A C-2 B^{2}\right) \partial_{B}+(2 A D-4 B C) \partial_{C}+(3 A E-6 B D) \partial_{D}+\ldots
$$

Thus we shall obtain the principiant generator by adding the second of Cayley's generators to six times the first. Either of Cayley's generators acting on a principiant would of course give an invariant in the large letters (that is, a principiant), but the combination we have used has special relation to the theory of the generation of principiants by differentiation. 


\section{LECTURE XXXII.}

I will now pass on to the consideration of the Principiant which, when equated to zero, gives the Differential Equation to the most general Algebraic Curve of any order.

The Differential Equation to a Conic (see the reference given [p. 380, above]) was obtained by Monge in the first decade of this century. This was followed by the determination, in 1868, by Mr Samuel Roberts, of the Differential Equation to the general Cubic (see Vol. x. p. 47 of Mathematical Questions and Solutions from the Educational Times). I do not consider that any substantial advance was made upon this by Mr Muir, in the Philosophical Magazine for February, 1886, except that he sets out explicitly the quantities to be eliminated in obtaining the final result. These may, of course, be collected from the processes indicated by $\mathrm{Mr}$ Roberts, but are not set forth by him. In speaking of the history of this part of the subject, I pass over M. Halphen's process for obtaining the Differential Equation to a Conic. It is very ingenious, like everything that proceeds from his pen, but, being founded on the solution of a quadratic equation, does not admit of being extended to forms of a higher degree, and consequently, viewed in the light of subsequent experience, must be regarded as faulty in point of method.

Let the Differential Equation to a curve of any order, when written in its simplest form, containing no extraneous factor, be $\chi=0$. It is convenient to give $\chi$ a single name; I call it the Criterion. The integral of the Criterion to a curve of order $n$ must contain as many arbitrary constants as there are ratios between the coefficients of a curve of the $n$th order. The number of these ratios being $\frac{n^{2}+3 n+2}{2}-1$, the order of the Criterion ought to be $\frac{n^{2}+3 n}{2}$

It must be independent of Perspective Projection, because projection does not affect the order of a curve. Hence it is a Principiant, and as such ought not (when $y$ is regarded as the dependent and $x$ as the independent variable) to contain either $x, y$ or $\frac{d y}{d x}$ (see Lecture XXIV. [p. 438, above]).

Let $U=0$ be an algebraical equation of the $n$th order between $x, y$. I write symbolically

$$
U=(p+q x+y)^{n}=u^{n},
$$

where the different powers and products of $p, q, 1$ which occur in the expan- 
sion of $u^{n}$ are considered as representing the different coefficients in $U$; so that, for example, if $n=3$ the coefficients of

$$
y^{3}, 3 y^{2} x, 3 y^{2}, 3 y x^{2}, 6 y x, 3 y, x^{3}, 3 x^{2}, 3 x, 1
$$

are represented by

$$
\text { 1, } q, \quad p, q^{2}, \quad p q, p^{2}, q^{3}, p q^{2}, p^{2} q, p^{3} .
$$

The number of terms in $U$ is

$$
1+2+3+\ldots+(n+1)=\frac{(n+1)(n+2)}{2}
$$

The number of these containing $y$ is

$$
1+2+3+\ldots+n=\frac{n(n+1)}{2} .
$$

To obtain the Differential Equation we equate to zero the Differential Derivatives of $U$ of all orders from $n+1$ to $\frac{1}{2}\left(n^{2}+3 n\right)$ inclusive, and from the $\frac{1}{2}\left(n^{2}+n\right)$ equations thus formed eliminate the $\frac{1}{2}\left(n^{2}+n\right)$ coefficients of the terms in $U$ containing $y$.

All the coefficients of pure powers of $x$ will obviously disappear under differentiation; for no power of $x$ higher than $x^{n}$ occurs in $U$, and no differential derivative of $U$ of lower order than $n+1$ is taken.

We thus find a differential equation of the order $\frac{1}{2}\left(n^{2}+3 n\right)$, free from all the $\frac{1}{2}\left(n^{2}+3 n+2\right)$ coefficients of $U$. This equation might conceivably contain $x, y$ and all the successive differential derivatives of $y$ with respect to $x$. But we know à priori that it ought not to contain either $x, y$ or $\frac{d y}{d x}$; and in fact we shall be able so to conduct the elimination that $x, y$ and $\frac{d y}{d x}$ appear only in the quantities to be eliminated and not in the final result.

Treating $u=p+q x+y$ as an ordinary algebraical quantity, we have, by Taylor's theorem,

$$
\frac{1}{1.2 .3 \ldots r} \cdot \frac{d^{r} u^{n}}{d x^{r}}=\text { co. } h^{r} \text { in }\left(u+u_{1} h+u_{2} \frac{h^{2}}{1.2}+u_{3} \frac{h^{3}}{1.2 .3}+\ldots\right)^{n},
$$

where $u_{1}, u_{2}, u_{3}, \ldots$ are the successive differential derivatives of $u$ with respect to $x$. And this result will remain true when for $u^{n}$ we write $U$, meaning thereby that $\frac{1}{1.2 .3 \ldots r} \cdot \frac{d^{r} U}{d x^{r}}$ will be the quantitative interpretation of the function of $u, u_{1}, u_{2}, \ldots$ which multiplies $h^{r}$ in the expansion of

$$
\left(u+u_{1} h+u_{2} \frac{h^{2}}{1.2}+\ldots\right)^{n}
$$

subject to the condition that this function shall be linear in the coefficients 
of $U$. This condition can be fulfilled in only one way, so that there is no ambiguity in such interpretation. Hence the equations obtained by equating to zero the successive differential derivatives of $U$ of all orders from $n+1$ to $\frac{1}{2}\left(n^{2}+3 n\right)$ inclusive may be written under the form

$$
\text { co. } h^{r} \text { in }\left(u+u_{1} h+u_{2} \frac{h^{2}}{1.2}+u_{3} \frac{h^{3}}{1.2 .3}+\ldots\right)^{n}=0
$$

where

$$
r=n+1, n+2, n+3, \ldots \frac{1}{2}\left(n^{2}+3 n\right) \text {. }
$$

Now, using $y_{1}, y_{2}, y_{3}, \ldots$ to denote the successive differential derivatives of $y$ with respect to $x$, we have

$$
u_{1}=q+y_{1}, \quad u_{2}=y_{2}, \quad u_{3}=y_{3}, \ldots,
$$

and, in general, $u_{i}=y_{i}$ when $i$ is any positive integer greater than 1 . Thus

$$
\text { co. } h^{r} \text { in }\left(u+u_{1} h+y_{2} \frac{h^{2}}{1.2}+y_{3} \frac{h^{3}}{1.2 .3}+\ldots\right)^{n}=0
$$

or, employing the usual modified derivatives $a, b, c, \ldots$,

$$
\text { co. } h^{r} \text { in }\left(u+u_{1} h+a h^{2}+b h^{3}+c h^{4}+\ldots\right)^{n}=0 .
$$

Writing now

$$
Q=a h^{2}+b h^{3}+c h^{4}+\ldots,
$$

and expanding $\left(u+u_{1} h+Q\right)^{n}$ in ascending powers of $Q$, we have

$$
\text { co. } h^{r} \text { in }\left\{\left(u+u_{1} h\right)^{n}+n\left(u+u_{1} h\right)^{n-1} Q+\frac{n(n-1)}{1.2}\left(u+u_{1} h\right)^{n-2} Q^{2}+\ldots\right\}=0
$$

where, remembering that $r>n$, the value of co. $h^{r}$ in $\left(u+u_{1} h\right)^{n}$ is zero; so that, omitting this term, we may write

$$
\text { co. } h^{r} \text { in }\left\{n\left(u+u_{1} h\right)^{n-1} Q+\frac{n(n-1)}{1.2}\left(u+u_{1} h\right)^{n-2} Q^{2}+\ldots+Q^{n}\right\}=0
$$

The quantities to be eliminated will now be combinations of the various powers of $u, u_{1}$ and 1 . Their number will be the same as that of the terms in $\left(u, u_{1}, 1\right)^{n-1}$, which is $\frac{1}{2}\left(n^{2}+n\right)$, the same number as that of the equations between which the elimination is to be performed.

We now use $(m . \mu)$ to denote the coefficient of $h^{m}$ in $Q^{\mu}$ (which, since

$$
Q=a h^{2}+b h^{3}+c h^{4}+\ldots,
$$

will be independent of the combinations of $u$ and $u_{1}$ to be eliminated), and in writing out the $\frac{1}{2}\left(n^{2}+n\right)$ equations which result from making the coefficients of $h^{n+1}, h^{n+2}, \ldots h^{\frac{n^{2}+3 n}{2}}$ in

$$
n\left(u+u_{1} h\right)^{n-1} Q+\frac{n(n-1)}{1.2}\left(u+u_{1} h\right)^{n-2} Q^{2}+\ldots+Q^{n}
$$


vanish, we arrange their terms according to ascending values of $m$ and $\mu$. Thus, making the coefficient of $h^{n+1}$ vanish, we find

$n u_{1}^{n-1}(2.1)+n(n-1) u_{1}^{n-2} u(3.1)+\frac{n(n-1)}{1.2} u_{1}^{n-2}(3.2)+\ldots+(n+1 . n)=0$,

and similarly, making the coefficient of $h^{n+2}$ vanish,

$n u_{1}^{n-1}(3.1)+n(n-1) u_{1}^{n-2} u(4.1)+\frac{n(n-1)}{1.2} u_{1}^{n-2}(4.2)+\ldots+(n+2 . n)=0$.

So in general the equation obtained by making the coefficient of $h^{n+\kappa}$ vanish consists of a series of numerical multiples (which are independent of the value of $\kappa)$ of $u_{1}^{n-\theta} u^{\theta-\eta}(\theta+\kappa, \eta)$ where $\eta$ has all values from 1 to $\theta$ inclusive, and $\theta$ all values from 1 to $n$ inclusive. Hence, by elimination, we find
(2.1) (3.1)
$(3.2)(4.1)$
$(4.2)$
$(5.1)(5.2)$
$(5.3)$
$(5.4) \ldots$
$\begin{array}{llllllllll}(3.1) & (4.1) & (4.2) & (5.1) & (5.2) & (5.3) & (6.1) & (6.2) & (6.3) & (6.4) \ldots\end{array}$
$\begin{array}{lllllllllll}(4.1) & (5.1) & (5.2) & (6.1) & (6.2) & (6.3) & (7.1) & (7.2) & (7.3) & (7.4) \ldots\end{array}$
$\begin{array}{llllllllll}(5.1) & (6.1) & (6.2) & (7.1) & (7.2) & (7.3) & (8.1) & (8.2) & (8.3) & (8.4) \ldots\end{array}$
$\begin{array}{lllllllllll}(6.1) & (7.1) & (7.2) & (8.1) & (8.2) & (8.3) & (9.1) & (9.2) & (9.3) & (9.4) \ldots\end{array}$

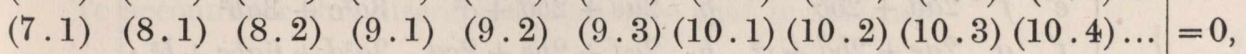
$(8.1)(9.1)(9.2)(10.1)(10.2)(10.3)(11.1)(11.2)(11.3)(11.4) \ldots$
$(9.1)(10.1)(10.2)(11.1)(11.2)(11.3)(12.1)(12.2)(12.3)(12.4) \ldots$
$(10.1)(11.1)(11.2)(12.1)(12.2)(12.3)(13.1)(13.2)(13.3)(13.4) \ldots$
$(11.1)(12.1)(12.2)(13.1)(13.2)(13.3)(14.1)(14.2)(14.3)(14.4) \ldots$

where the determinant on the left-hand side, consisting of $\frac{1}{2}\left(n^{2}+n\right)$ rows and columns, is the Criterion of the curve of the $n$th order.

Thus in the case of the Cubic Criterion, which we shall specially consider, we have $n=3$, and the elimination of $3 u_{1}^{2}, 6 u_{1} u, 3 u_{1}, 3 u^{2}, 3 u$ and 1 between the six equations

$$
\begin{aligned}
& 3 u_{1}^{2}(2.1)+6 u_{1} u(3.1)+3 u_{1}(3.2)+3 u^{2}(4.1)+3 u(4.2)+(4.3)=0 \\
& 3 u_{1}^{2}(3.1)+6 u_{1} u(4.1)+3 u_{1}(4.2)+3 u^{2}(5.1)+3 u(5.2)+(5.3)=0 \\
& 3 u_{1}^{2}(4.1)+6 u_{1} u(5.1)+3 u_{1}(5.2)+3 u^{2}(6.1)+3 u(6.2)+(6.3)=0 \\
& 3 u_{1}^{2}(5.1)+6 u_{1} u(6.1)+3 u_{1}(6.2)+3 u^{2}(7.1)+3 u(7.2)+(7.3)=0 \\
& 3 u_{1}^{2}(6.1)+6 u_{1} u(7.1)+3 u_{1}(7.2)+3 u^{2}(8.1)+3 u(8.2)+(8.3)=0 \\
& 3 u_{1}^{2}(7.1)+6 u_{1} u(8.1)+3 u_{1}(8.2)+3 u^{2}(9.1)+3 u(9.2)+(9.3)=0
\end{aligned}
$$

gives the Cubic Criterion in the form of the determinant

$\left|\begin{array}{llllll}(2.1) & (3.1) & (3.2) & (4.1) & (4.2) & (4.3) \\ (3.1) & (4.1) & (4.2) & (5.1) & (5.2) & (5.3) \\ (4.1) & (5.1) & (5.2) & (6.1) & (6.2) & (6.3) \\ (5.1) & (6.1) & (6.2) & (7.1) & (7.2) & (7.3) \\ (6.1) & (7.1) & (7.2) & (8.1) & (8.2) & (8.3) \\ (7.1) & (8.1) & (8.2) & (9.1) & (9.2) & (9.3)\end{array}\right|$.


Remembering that

$$
(m \cdot \mu)=\operatorname{co} . h^{m} \text { in }\left(a h^{2}+b h^{3}+c h^{4}+\ldots\right)^{\mu},
$$

it is easy to express the Criterion explicitly in terms of $a, b, c, \ldots$.

Thus, since

$$
\begin{aligned}
\left(a h^{2}+b h^{3}+c h^{4}+\ldots\right)^{2}= & a^{2} h^{4}+2 a b h^{5}+\left(2 a c+b^{2}\right) h^{6}+(2 a d+2 b c) h^{7} \\
& +\left(2 a e+2 b d+c^{2}\right) h^{8}+(2 a f+2 b e+2 c d) h^{9}+\ldots
\end{aligned}
$$

and

$$
\begin{aligned}
\left(a h^{2}+b h^{3}+c h^{4}+\ldots\right)^{3}=a^{3} h^{6}+3 a^{2} b h^{7}+ & \left(3 a^{2} c+3 a b^{2}\right) h^{8} \\
& +\left(3 a^{2} d+6 a b c+b^{3}\right) h^{9}+\ldots
\end{aligned}
$$

the Cubic Criterion may be written in the form

$$
\left|\begin{array}{llllll}
a & b & 0 & c & a^{2} & 0 \\
b & c & a^{2} & d & 2 a b & 0 \\
c & d & 2 a b & e & 2 a c+b^{2} & a^{3} \\
d & e & 2 a c+b^{2} & f & 2 a d+2 b c & 3 a^{2} b \\
e & f & 2 a d+2 b c & g & 2 a e+2 b d+c^{2} & 3 a^{2} c+3 a b^{2} \\
f & g & 2 a e+2 b d+c^{2} & h & 2 a f+2 b e+2 c d & 3 a^{2} d+6 a b c+b^{3}
\end{array}\right|
$$

in which it was originally obtained by Mr Roberts.

M. Halphen has remarked that the minor of $h$ in the Cubic Criterion is the Principiant which he calls $\Delta$ (our $A C-B^{2}$ ) multiplied by $a$ (see p. 50 of his Thèse).

We proceed to determine the degree and weight of the Criterion of the curve of the $n$th order. These are the same as the degree and weight of its diagonal

$(2.1)(4.1)(5.2)(7.1)(8.2)(9.3)(11.1)(12.2)(13.3)(14.4) \ldots$,

which consists of $\frac{1}{2}\left(n^{2}+n\right)$ factors, separable into $n$ groups,

(2.1), (4.1)(5.2), (7.1)(8.2)(9.3), (11.1)(12.2)(13.3)(14.4), .

containing $1,2,3,4, \ldots n$ factors respectively. Now,

$$
\begin{aligned}
(m . \mu) & =\text { co. } h^{m} \text { in }\left(a h^{2}+b h^{3}+c h^{4}+\ldots\right)^{\mu} \\
& =\text { co. } h^{m-2 \mu} \text { in }\left(a+b h+c h^{2}+\ldots\right)^{\mu},
\end{aligned}
$$

and consequently $(m . \mu)$ is of degree $\mu$ and weight $m-2 \mu$. Hence the degree of the Criterion (found by adding together the second numbers of the duads which occur in the diagonal) is

$$
\begin{aligned}
1+(1+2)+(1+ & 2+3)+(1+2+3+4)+\ldots+(1+2+3+\ldots+n) \\
& =1+3+6+10+\ldots+\frac{n^{2}+n}{2} \\
& =\frac{n(n+1)(n+2)}{6} .
\end{aligned}
$$


To find the weight of the Criterion, we begin by arranging the factors of its diagonal according to their weight. This is done by writing each group of factors in reverse order, so that the diagonal is written thus:

$(2.1)(5.2)(4.1)(9.3)(8.2)(7.1)(14.4)(13.3)(12.2)(11.1) \ldots$

The weights of the factors are now seen to be $0,1,2,3, \ldots \frac{n^{2}+n}{2}-1$; there being $\frac{1}{2}\left(n^{2}+n\right)$ factors in the diagonal, one of them of zero weight. Hence the weight of the Criterion is

$$
\begin{gathered}
1+2+3+\ldots+\left(\frac{n^{2}+n}{2}-1\right) \\
=\frac{\left(\frac{n^{2}+n}{2}-1\right) \frac{n^{2}+n}{2}}{2}=\frac{(n-1) n(n+1)(n+2)}{8} .
\end{gathered}
$$

If, in the above formulae, we make $n=2$, we shall find that the degree is 4 and the weight 3 , whereas the Mongian $a^{2} d-3 a b c+2 b^{3}$ (which is the Criterion of the second order) is of degree 3 and weight 3.

To account for this discrepancy, observe that in this case

$$
\begin{array}{lll}
(2.1) & (3.1) & (3.2) \\
(3.1) & (4.1) & (4.2) \\
(4.1) & (5.1) & (5.2)
\end{array}|=| \begin{array}{lll}
a & b & 0 \\
b & c & a^{2} \\
c & d & 2 a b
\end{array} \mid,
$$

which is divisible by $a$, the other factor being the Mongian, as may easily be verified. This is the only case in which the determinant expression for the Criterion contains an irrelevant factor.

To express the Cubic Criterion in terms of $a, A, B, C, D, E$, we first remark that its degree is $\frac{3.4 .5}{6}=10$, and its weight $\frac{2.3 .4 .5}{8}=15$. Thus the Cubic Criterion is expressible as the product of $a^{-5}(10-15=-5)$ into a function of the capital letters, which we determine by the usual method of substituting for

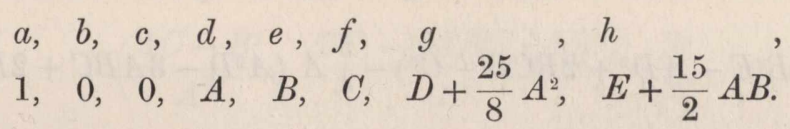

When these substitutions are made, the Cubic Criterion becomes

s. IV.

$\left|\begin{array}{llllll}1 & 0 & 0 & 0 & 1 & 0 \\ 0 & 0 & 1 & A & 0 & 0 \\ 0 & A & 0 & B & 0 & 1 \\ A & B & 0 & C & 2 A & 0 \\ B & C & 2 A & D+\frac{25}{8} A^{2} & 2 B & 0 \\ C & D+\frac{25}{8} A^{2} & 2 B & E+\frac{15}{2} A B & 2 C & 3 A\end{array}\right|$


Subtracting the first column of this determinant from the fifth and reducing, we obtain

$$
\left|\begin{array}{lllll}
0 & 1 & A & 0 & 0 \\
A & 0 & B & 0 & 1 \\
B & 0 & C & A & 0 \\
C & 2 A & D+\frac{25}{8} A^{2} & B & 0 \\
D+\frac{25}{8} A^{2} & 2 B & E+\frac{15}{2} A B & C & 3 A
\end{array}\right|
$$

Again, subtracting the second column multiplied by $A$ from the third and reducing, there results

$$
-\left|\begin{array}{llll}
A & B & 0 & 1 \\
B & C & A & 0 \\
C & D+\frac{9}{8} A^{2} & B & 0 \\
D+\frac{25}{8} A^{2} & E+\frac{11}{2} A B & C & 3 A
\end{array}\right|
$$

which, after subtracting the first row multiplied by $3 A$ from the last and reducing, becomes

$$
\begin{gathered}
\quad \begin{array}{ccc}
B & C & A \\
C & D+\frac{9}{8} A^{2} & B \\
D+\frac{1}{8} A^{2} & E+\frac{5}{2} A B & C
\end{array} \mid \\
=B\left(C D+\frac{9}{8} A^{2} C-B E-\frac{5}{2} A B^{2}\right)+C\left(B D+\frac{1}{8} A^{2} B-C^{2}\right) \\
+A\left(C E+\frac{5}{2} A B C-D^{2}-\frac{5}{4} A^{2} D-\frac{9}{64} A^{4}\right) \\
=\left(A C E-B^{2} E-A D^{2}+2 B C D-C^{3}\right)-\frac{5}{4} A\left(A^{2} D-3 A B C+2 B^{3}\right)-\frac{9}{64} A^{5} .
\end{gathered}
$$

This expression, which is of degree-weight 15.15 , instead of 10.15 , must be divided by $a^{5}$ to give the correct value of the Cubic Criterion. 


\section{LECTURE XXXIII.}

In this Lecture it is proposed to investigate the differential equation of a cubic curve having a given absolute invariant $\frac{S^{3}}{T^{2}}$.

Since the value of $\frac{S^{3}}{T^{2}}$ is the same for any homographic transformation of the cubic as for the original curve, the differential equation in question must be of the form

$$
\text { Plenarily absolute principiant }=\frac{S^{3}}{T^{2}} .
$$

This equation is (as we see at once by differentiating it) the integral of another of the form

$$
\text { Principiant }=0 \text {, }
$$

which is satisfied, independently of the value of the absolute invariant, at ail points on a perfectly general cubic.

Now, the differential equation of the general cubic is of the 9 th order, and when expressed in terms of $A, B, C, \ldots$ contains no letter beyond $E$. Hence the integral of this equation, which we are in search of, will be of the 8th order and will contain no capital letter beyond $D$.

When no letters beyond $D$ are involved, all plenarily absolute principiants are functions of the two fundamental, or protomorphic, ones,

$$
\frac{A C-B^{2}}{A^{\frac{8}{3}}}, \frac{A^{2} D-3 A B C+2 B^{3}}{A^{4}} \text {. }
$$

Thus the differential equation of a cubic with a given absolute invariant is of the form

$$
F\left(\frac{A C-B^{2}}{A^{\frac{s}{3}}}, \frac{A^{2} D-3 A B C+2 B^{3}}{A^{4}}\right)=\frac{S^{3}}{T^{2}} .
$$

M. Halphen actually integrates the differential equation of the general cubic, which he shows (on p. 52 of his Thèse sur les Invariants Différentiels) may be put under the form

$$
\xi \zeta d \xi+\left\{\zeta-\frac{3}{8}(\xi+3)(\xi+27)\right\} d \zeta=0
$$

where, in our notation,

$$
\xi=\frac{24\left(A^{2} D-3 A B C+2 B^{3}\right)}{A^{4}}, \quad \zeta=\frac{288\left(A C-B^{2}\right)^{3}}{A^{8}} .
$$


The integral of this equation, which M. Halphen obtains partly from geometrical considerations, involves an arbitrary parameter depending on $\frac{S^{3}}{T^{2}}$. His result is as follows :

where

$$
R^{2}=h Q^{3}
$$

$$
\begin{gathered}
2^{9} R=2^{9} \zeta^{3}+2^{6} \cdot 3\left[\left(\xi-3^{2}\right)^{2}+2^{4} .3^{4}\right] \zeta^{2}+2^{3} \cdot 3\left(\xi+3^{3}\right)^{3}\left(\xi-3^{2} .5\right) \zeta+\left(\xi+3^{3}\right)^{6}, \\
\text { and } \\
2^{6} Q=2^{6} \zeta^{2}+2^{4}\left(\xi+3^{3}\right)\left(\xi-3^{2} .5\right) \zeta+\left(\xi+3^{3}\right)^{4}, \\
T^{2}-64 h S^{3}=0 .
\end{gathered}
$$

(Two misprints, which are here corrected, occur in the expression for $R$ as given on p. 54 of the Thèse.)

In this result the invariant $S$ differs in sign from the invariant usually denoted by that letter. Thus the discriminant is $T^{2}-64 S^{3}$ instead of $T^{2}+64 S^{3}$.

When $h=1$ the discriminant vanishes and the differential equation becomes

$$
R^{2}-Q^{3}=0 .
$$

This is divisible by a numerical multiple of $\zeta^{3}$; in fact,

$$
R^{2}=Q^{3}+2^{3} \cdot 3^{5} \zeta^{3} P,
$$

where

$$
2^{6} P \equiv\left(2^{3} \zeta+\xi^{2}-2.3^{3} \xi-3^{5}\right)^{2}+2^{6} \cdot 3 \xi^{3}=0
$$

is the differential equation of a nodal cubic, previously obtained by Halphen.

It is from a knowledge of the fact that $P=0$ and another algebraic relation between $\xi$ and $\zeta$, which he finds by trial to be $Q=0$, constitute two particular integrals of the differential equation to the general cubic, that he arrives, not by any regular method but by repeated strokes of penetrative genius, at the general integral

$$
R^{2}=h Q^{3} \text {. }
$$

In establishing the relation $T^{2}-64 h S^{3}=0$ he supposes that, by means of the equation to the cubic and its differentials as far as the 8th order inclusive, the coefficients of the cubic have been expressed in terms of the variables $x, y$ and the derivatives of $y$ with respect to $x$ up to the 8th order, and that the values thus obtained for the coefficients have been substituted in Aronhold's $S$ and $T$.

The abbreviations introduced by the use of our notation enable us to actually perform this calculation, which would otherwise be impracticable in consequence of the enormous amount of labour required; and we shall use this method to obtain the plenarily absolute principiant which, equated to $\frac{S^{3}}{T^{2}}$, gives the differential equation to a cubic with a known absolute invariant. 
Using the symbolic notation explained in Lecture XXXII. [above, p. 492], the equation of the cubic and its first eight differentials are

$$
\begin{aligned}
& u^{3}=0, \\
& u^{2} u_{1}=0, \\
& 2 u u_{1}{ }^{2}+u^{2} u_{2}=0, \\
& 2 u_{1}{ }^{3}+6 u u_{1} u_{2}+u^{2} u_{3}=0, \\
& 3 u_{1}{ }^{2}(2.1)+6 u_{1} u(3.1)+3 u_{1}(3.2)+3 u^{2}(4.1)+3 u(4.2)+(4.3)=0, \\
& 3 u_{1}^{2}(3.1)+6 u_{1} u(4.1)+3 u_{1}(4.2)+3 u^{2}(5.1)+3 u(5.2)+(5.3)=0, \\
& 3 u_{1}{ }^{2}(4.1)+6 u_{1} u(5.1)+3 u_{1}(5.2)+3 u^{2}(6.1)+3 u(6.2)+(6.3)=0, \\
& 3 u_{1}{ }^{2}(5.1)+6 u_{1} u(6.1)+3 u_{1}(6.2)+3 u^{2}(7.1)+3 u(7.2)+(7.3)=0, \\
& 3 u_{1}{ }^{2}(6.1)+6 u_{1} u(7.1)+3 u_{1}(7.2)+3 u^{2}(8.1)+3 u(8.2)+(8.3)=0,
\end{aligned}
$$

where

$$
u=p+q x+y, \quad u_{1}=q+t, \quad u_{2}=2 a, \quad u_{3}=6 b
$$

as usual,

$$
t=\frac{d y}{d x}, \quad a=\frac{1}{2} \cdot \frac{d^{2} y}{d x^{2}}, \quad b=\frac{1}{6} \cdot \frac{d^{3} y}{d x^{3}}, \ldots ;
$$

$(m . \mu)$ denotes the coefficient of $h^{m}$ in $\left(a h^{2}+b h^{3}+c h^{4}+\ldots\right)^{\mu}$; and if, as in Salmon's Higher Plane Curves (2nd edit., p. 187), the equation of the cubic is taken to be

$$
r+3 a_{0} x+3 a_{1} y+3 b_{0} x^{2}+6 b_{1} x y+3 b_{2} y^{2}+c_{0} x^{3}+3 c_{1} x^{2} y+3 c_{2} x y^{2}+c_{3} y^{3}=0,
$$

then, in the above equations, the symbols

stand for

$$
\begin{aligned}
& p^{3}, p^{2} q, p^{2}, p q^{2}, p q, p, q^{3}, q^{2}, q, 1 \\
& r, a_{0}, a_{1}, b_{0}, b_{1}, b_{2}, c_{0}, c_{1}, c_{2}, c_{3} .
\end{aligned}
$$

These nine equations are sufficient to determine the values of the coefficients of the cubic which have to be substituted in $\frac{S^{3}}{T^{2}}$ in order to obtain our differential equation, which will be, as we have seen, of the form

$$
F\left(\frac{A C-B^{2}}{A^{\frac{8}{3}}}, \frac{A^{2} D-3 A B C+2 B^{3}}{A^{4}}\right)=\frac{S^{3}}{T^{2}} .
$$

Since this equation contains nothing which involves $x, y$, or $t$, these letters must have disappeared spontaneously in the process of forming it, and consequently we may, at any stage of the work, give $x, y$, and $t$ any arbitrary values without thereby affecting the result. Let, then,

$$
x=0, y=0, t=0 \text {, so that } u=p, u_{1}=q, u_{2}=2 a, u_{3}=6 b \text {, }
$$

and the first four equations become

$$
\begin{gathered}
u^{3}=p^{3}=r=0, \\
u^{2} u_{1}=p^{2} q=a_{0}=0, \\
\frac{1}{2}\left(2 u u_{1}^{2}+u^{2} u_{2}\right)=p q^{2}+p^{2} a=b_{0}+a_{1} a=0, \\
\frac{1}{2}\left(2 u_{1}^{3}+6 u u_{1} u_{2}+u^{2} u_{3}\right)=q^{3}+6 p q a+3 p^{2} b=c_{0}+6 b_{1} a+3 a_{1} b=0 .
\end{gathered}
$$


Writing in the last five equations

we have

$$
\begin{array}{r}
u_{1}^{2}=q^{2}=c_{1}, \\
u_{1} u=p q=b_{1}, \\
u_{1}=q=c_{2}, \\
u^{2}=p^{2}=a_{1}, \\
u=p=b_{2}, \\
1=c_{3},
\end{array}
$$

$$
\begin{aligned}
& 3 c_{1}(2.1)+6 b_{1}(3.1)+3 c_{2}(3.2)+3 a_{1}(4.1)+3 b_{2}(4.2)+c_{3}(4.3)=0 \\
& 3 c_{1}(3.1)+6 b_{1}(4.1)+3 c_{2}(4.2)+3 a_{1}(5.1)+3 b_{2}(5.2)+c_{3}(5.3)=0 \\
& 3 c_{1}(4.1)+6 b_{1}(5.1)+3 c_{2}(5.2)+3 a_{1}(6.1)+3 b_{2}(6.2)+c_{3}(6.3)=0 \\
& 3 c_{1}(5.1)+6 b_{1}(6.1)+3 c_{2}(6.2)+3 a_{1}(7.1)+3 b_{2}(7.2)+c_{3}(7.3)=0 \\
& 3 c_{1}(6.1)+6 b_{1}(7.1)+3 c_{2}(7.2)+3 a_{1}(8.1)+3 b_{2}(8.2)+c_{3}(8.3)=0 *
\end{aligned}
$$

Substituting in $\frac{S^{3}}{T^{2}}$ for $r, a_{0}, b_{0}, c_{0}$ their values given by the equations

$$
r=0, \quad a_{0}=0, \quad b_{0}+a_{1} a=0, \quad c_{0}+6 b_{1} a+3 a_{1} b=0,
$$

and for the mutual ratios of $a_{1}, b_{1}, b_{2}, c_{1}, c_{2}, c_{3}$ their values found by solving the last five equations, we obtain the differential equation required. $r=0$,

Referring to Salmon's Higher Plane Curves, p. 188, we see that, when

$$
\begin{aligned}
& S=\left(c^{2} a^{2}\right)+\left(c b^{2} a\right)-\left(b^{2}\right)^{2}, \\
& T=4\left(c^{3} a^{3}\right)-3\left(c^{2} b^{2} a^{2}\right)-12\left(b^{2}\right)\left(c b^{2} a\right)+8\left(b^{2}\right)^{3},
\end{aligned}
$$

where $\left(c^{2} a^{2}\right),\left(c b^{2} a\right), \ldots$ are functions of $a_{0}, a_{1}, b_{0}, b_{1}, b_{2}, c_{0}, c_{1}, c_{2}, c_{3}$, which, when $a_{0}=0$, become

$$
\begin{aligned}
\left(c^{2} a^{2}\right) & =\left(c_{0} c_{2}-c_{1}^{2}\right) a_{1}^{2}, \\
\left(c b^{2} a\right) & =\left(b_{0}^{2} c_{3}-3 b_{0} b_{1} c_{2}+b_{0} b_{2} c_{1}+2 b_{1}{ }^{2} c_{1}-b_{1} b_{2} c_{0}\right) a_{1}, \\
\left(b^{2}\right) & =b_{0} b_{2}-b_{1}{ }^{2} \\
\left(c^{3} a^{3}\right) & =\left(c_{0}{ }^{2} c_{3}-3 c_{0} c_{1} c_{2}+2 c_{1}^{3}\right) a_{1}^{3} \\
\left(c^{2} b^{2} a^{2}\right) & =\left(c_{0}{ }^{2} b_{2}{ }^{2}-4 c_{0} c_{1} b_{1} b_{2}-2 c_{0} c_{2} b_{0} b_{2}-4 c_{0} c_{2} b_{1}{ }^{2}+8 c_{0} c_{3} b_{0} b_{1}\right. \\
& \left.+8 c_{1}^{2} b_{1}{ }^{2}+4 c_{1}{ }^{2} b_{0} b_{2}-12 c_{1} c_{2} b_{0} b_{1}-8 c_{1} c_{3} b_{0}{ }^{2}+9 c_{2}{ }^{2} b_{0}{ }^{2}\right) a_{1}{ }^{2} .
\end{aligned}
$$

We have now reached a point at which the work will be greatly facilitated by the introduction of the capital letters $A, B, C, D$. This is usually done by writing for

$$
\begin{aligned}
& a, b, c, d, e, f, g \\
& 1,0,0, A, B, C, D+\frac{25}{8} A^{2}
\end{aligned}
$$

* These equations are only set out for the sake of distinctness; when our abbreviations are introduced, only two terms survive in the first three, and only three terms in the last two of these five equations. 
But in the present instance we may make a further simplification by writing

$$
A=1, B=0, C=C_{1}, \quad D=D_{1},
$$

for the only effect of this will be to make the final result take the form

$$
F\left(C_{1}, D_{1}\right)=\frac{S^{3}}{T^{2}}
$$

instead of $\quad F\left(\frac{A C-B^{2}}{A^{\frac{8}{3}}}, \frac{A^{2} D-3 A B C+2 B^{3}}{A^{4}}\right)=\frac{S^{3}}{T^{2}}$.

The form of the function will not be affected by writing in it $A=1, B=0$, and the letters $A, B$ can be restored at pleasure by making

$$
C_{1}=\frac{A C-B^{2}}{A^{\frac{8}{3}}}, \quad D_{1}=\frac{A^{2} D-3 A B C+2 B^{3}}{A^{4}} .
$$

Hence we may write for

$$
\begin{aligned}
& a, b, c, d, e, f, g \\
& 1,0,0,1,0, C_{1}, D_{1}+\frac{25}{8} .
\end{aligned}
$$

Instead of the coefficient of

$$
h^{m} \text { in }\left(a h^{2}+b h^{3}+c h^{4}+\ldots\right)^{\mu}
$$

$(m \cdot \mu)$ will now signify

Thus we have

$$
\text { co. } h^{m} \text { in }\left\{h^{2}+h^{5}+C_{1} h^{7}+\left(D_{1}+\frac{25}{8}\right) h^{8}\right\}^{\mu} \text {. }
$$

$$
\begin{array}{lll}
(2.1)=1 & \\
(3.1)=0 & (3.2)=0 & \\
(4.1)=0 & (4 \cdot 2)=1 & (4.3)=0 \\
(5.1)=1 & (5.2)=0 & (5.3)=0 \\
(6.1)=0 & (6.2)=0 & (6.3)=1 \\
(7.1)=C_{1} & (7.2)=2 & (7.3)=0 \\
(8.1)=D_{1}+\frac{25}{8} & (8.2)=0 & (8.3)=0
\end{array}
$$

Hence the equations which give $a_{1}, b_{1}, b_{2}, c_{1}, c_{2}, c_{3}$ become

$$
\begin{array}{r}
c_{1}+b_{2}=0 \\
c_{2}+a_{1}=0 \\
6 b_{1}+c_{3}=0 \\
c_{1}+a_{1} C_{1}+2 b_{2}=0 \\
2 b_{1} C_{1}+2 c_{2}+a_{1}\left(D_{1}+\frac{25}{8}\right)=0
\end{array}
$$

From the first four of these, coupled with the equations

$$
b_{0}+a_{1}=0, \quad c_{0}+6 b_{1}=0,
$$


obtained by making $\alpha=1$ and $b=0$ in the original equations which give $b_{0}, c_{0}$, we find

$$
\begin{aligned}
& c_{0}=c_{3}=-6 b_{1}, \\
& c_{1}=-b_{2}=-C_{1}^{2}, \\
& c_{2}=b_{0}=-a_{1}=C_{1},
\end{aligned}
$$

by assuming $a_{1}=-C_{1}$ (which we are at liberty to do since any one of the coefficients may be chosen arbitrarily).

The last equation then gives

$$
b_{1}=\frac{D_{1}}{2}+\frac{9}{16}
$$

Substituting these values in the previously given expressions for $\left(c^{2} \alpha^{2}\right)$, $\left(c b^{2} a\right), \ldots$ we have

$$
\begin{aligned}
\left(c^{2} a^{2}\right) & =-\left(6 b_{1}+C_{1}^{3}\right) C_{1}^{3}, \\
\left(c b^{2} a\right) & =-\left(4 b_{1}^{2}-9 b_{1}-C_{1}^{3}\right) C_{1}^{3}, \\
\left(b^{2}\right) & =C_{1}^{3}-b_{1}^{2}, \\
\left(c^{3} a^{3}\right) & =\left(216 b_{1}^{3}+18 b_{1} C_{1}^{3}+2 C_{1}^{6}\right) C_{1}^{3}, \\
\left(c^{2} b^{2} a^{2}\right) & =\left(312 b_{1}^{3}+20 b_{1}{ }^{2} C_{1}^{3}-24 b_{1} C_{1}^{3}+9 C_{1}^{3}+4 C_{1}{ }^{6}\right) C_{1}^{3} .
\end{aligned}
$$

Hence $\quad S=\left(c^{2} a^{2}\right)+\left(c b^{2} a\right)-\left(b^{2}\right)^{2}$

$$
=-C_{1}{ }^{6}+3 b_{1} C_{1}{ }^{3}-2 b_{1}{ }^{2} C_{1}{ }^{3}-b_{1}{ }^{4} \text {, }
$$

and

$$
\begin{aligned}
T & =4\left(c^{3} a^{3}\right)-3\left(c^{2} b^{2} a^{2}\right)-12\left(b^{2}\right)\left(c b^{2} a\right)+8\left(b^{2}\right)^{3} \\
& =-8 C_{1}{ }^{9}-3\left(8 b_{1}{ }^{2}-12 b_{1}+9\right) C_{1}{ }^{6}-12 b_{1}{ }^{3}\left(2 b_{1}-3\right) C_{1}{ }^{3}-8 b_{1}{ }^{6} .
\end{aligned}
$$

To express $S$ and $T$ in terms of $A, B, C, D$, we write

$$
C_{1}=\frac{A C-B^{2}}{A^{\frac{8}{3}}}, \quad b_{1}=\frac{D_{1}}{2}+\frac{9}{16}=\frac{A^{2} D-3 A B C+2 B^{3}}{2 A^{4}}+\frac{9}{16},
$$

or, if we use Halphen's notation in which

$$
\zeta=\frac{288\left(A C-B^{2}\right)^{3}}{A^{8}}, \quad \xi=\frac{24\left(A^{2} D-3 A B C+2 B^{3}\right)}{A^{4}},
$$

we have

$$
2^{5} \cdot 3^{2} C_{1}^{3}=\zeta, \quad 2^{4} \cdot 3 b_{1}=\xi+3^{3},
$$

and consequently,

$$
2^{3} \cdot 3\left(2 b_{1}-3\right)=\xi-3^{2} .5,
$$

$$
2^{5} \cdot 3^{2}\left(8 b_{1}{ }^{2}-12 b_{1}+9\right)=\left(2^{4} \cdot 3 b_{1}-2^{2} \cdot 3^{2}\right)^{2}+2^{4} \cdot 3^{4}=\left(\xi-3^{2}\right)^{2}+2^{4} \cdot 3^{4}
$$

Hence

$$
\begin{aligned}
-2^{16} \cdot 3^{4} S= & 2^{16} \cdot 3^{4} C_{1}{ }^{6}+2^{16} \cdot 3^{4} b_{1}\left(2 b_{1}-3\right) C_{1}{ }^{3}+2^{16} \cdot 3^{4} b_{1}{ }^{4} \\
= & 2^{6} \zeta^{2}+2^{4}\left(\xi+3^{3}\right)\left(\xi-3^{2} \cdot 5\right) \zeta+\left(\xi+3^{3}\right)^{4} \\
-2^{21} \cdot 3^{6} T= & 2^{24} \cdot 3^{6} C_{1}^{9}+2^{21} \cdot 3^{7}\left(8 b_{1}{ }^{2}-12 b_{1}+9\right) C_{1}{ }^{6} \\
& +2^{23} \cdot 3^{7} b_{1}{ }^{3}\left(2 b_{1}-3\right) C_{1}{ }^{3}+2^{24} \cdot 3^{6} b_{1}{ }^{6} \\
= & 2^{9} \zeta^{3}+2^{6} \cdot 3\left[\left(\xi-3^{2}\right)^{2}+2^{4} \cdot 3^{4}\right] \zeta^{2} \\
& +2^{3} \cdot 3\left(\xi+3^{3}\right)^{3}\left(\xi-3^{2} \cdot 5\right) \zeta+\left(\xi+3^{3}\right)^{6}
\end{aligned}
$$


where the expressions on the right-hand side are $2{ }^{6} Q$ and $2{ }^{9} R$ in Halphen's notation. Thus

so that

$$
\begin{gathered}
-2^{10} \cdot 3^{4} S=Q, \quad-2^{12} \cdot 3^{6} T=R \\
\frac{Q^{3}}{R^{2}}=-\frac{2^{30} \cdot 3^{12} S^{3}}{2^{24} \cdot 3^{12} T^{2}}=-\frac{64 S^{3}}{T^{2}}
\end{gathered}
$$

This result agrees exactly with Halphen's, if we remember that his $S$ is taken with a different sign from ours.

Since

$$
b_{1}=\frac{D_{1}}{2}+\frac{9}{16}=\frac{A^{2} D-3 A B C+2 B^{3}}{2 A^{4}}+\frac{3^{2}}{2^{4}},
$$

we may write

$$
\Phi=2^{4} A^{4} b_{1}=2^{3}\left(A^{2} D-3 A B C+2 B^{3}\right)+3^{2} A^{4},
$$

and in like manner

Now

$$
\Psi=A^{8} C_{1}^{3}=\left(A C-B^{2}\right)^{3} .
$$$$
2^{8} A^{8}\left(b_{1}^{2}+C_{1}^{3}\right)=\Phi^{2}+2^{8} \Psi,
$$

which is divisible by $\boldsymbol{A}^{2}$. Hence if

$$
\Phi^{2}+2^{8} \Psi=A^{2} \Theta
$$

we have $\quad \Theta=2^{8} A^{6}\left(b_{1}{ }^{2}+C_{1}{ }^{3}\right)$

$$
\begin{aligned}
=2^{6}\left(A^{2} D^{2}-6 A B C D+\right. & \left.4 A C^{3}+4 B^{3} D-3 B^{2} C^{2}\right) \\
& +2^{4} \cdot 3^{2} A^{2}\left(A^{2} D-3 A B C+2 B^{3}\right)+3^{4} A^{6} .
\end{aligned}
$$

The equations which give $S$ and $T$ in terms of $b_{1}$ and $C_{1}$ may be written

$$
\begin{aligned}
& -S=\left(b_{1}{ }^{2}+C_{1}{ }^{3}\right)^{2}-3 b_{1} C_{1}^{3}, \\
& -T=2^{3}\left(b_{1}{ }^{2}+C_{1}^{3}\right)^{3}-2^{2} \cdot 3^{2}\left(b_{1}{ }^{2}+C_{1}{ }^{3}\right) b_{1} C_{1}{ }^{3}+3^{3} C_{1}{ }^{6},
\end{aligned}
$$

and consequently,

$$
\begin{aligned}
& -2^{16} A^{12} S=\Theta^{2}-2^{12} \cdot 3 \Phi \Psi, \\
& -2^{21} A^{18} T=\Theta^{3}-2^{11} \cdot 3^{2} \Theta \Phi \Psi+2^{21} \cdot 3^{3} A^{2} \Psi^{2},
\end{aligned}
$$

where $\Theta, \Phi, \Psi$ are the rational integral principiants

$$
\begin{aligned}
\Theta & =2^{6}\left(A^{2} D^{2}-6 A B C D+4 A C^{3}+4 B^{3} D-3 B^{2} C^{2}\right) \\
& +2^{4} \cdot 3^{2} A^{2}\left(A^{2} D-3 A B C+2 B^{3}\right)+3^{4} A^{6}, \\
\Phi & =2^{3}\left(A^{2} D-3 A B C+2 B^{3}\right)+3^{2} A^{4}, \\
\Psi & =\left(A C-B^{2}\right)^{3},
\end{aligned}
$$

which, as we have seen, are connected by the relation

$$
\Phi^{2}+2^{8} \Psi=A^{2} \Theta .
$$

The differential equation of cubics with a given absolute invariant is

$$
\frac{\left(\Theta^{2}-2^{12} \cdot 3 \Phi \Psi\right)^{3}}{\left(\Theta^{3}-2^{11} \cdot 3^{2} \Theta \Phi \Psi+2^{21} \cdot 3^{3} A^{2} \Psi^{2}\right)^{2}}=-\frac{2^{6} S^{3}}{T^{2}}
$$

or, as it may also be written,

$$
\left(\Theta^{2}-2^{12} \cdot 3 \Phi \Psi\right)^{3} T^{2}+2^{6} S^{3}\left(\Theta^{3}-2^{11} \cdot 3^{2} \Theta \Phi \Psi+2^{21} \cdot 3^{3} A^{2} \Psi^{2}\right)^{2}=0 .
$$


For a nodal cubic, the discriminant $T^{2}+2{ }^{6} S^{3}$ vanishes. Hence the differential equation of a nodal cubic is

$$
\left(\Theta^{3}-2^{11} \cdot 3^{2} \Theta \Phi \Psi+2^{21} \cdot 3^{3} A^{2} \Psi^{2}\right)^{2}-\left(\Theta^{2}-2^{12} .3 \Phi \Psi\right)^{3}=0 .
$$

When expanded, and divided by $2^{22} \cdot 3^{3} \Psi^{2}$, this reduces to

$$
A^{2} \Theta^{3}-\Theta^{2} \Phi^{2}-2^{11} \cdot 3^{2} A^{2} \Theta \Phi \Psi+2^{14} \Phi^{3} \Psi+2^{20} \cdot 3^{3} A^{4} \Psi^{2}=0,
$$

which (since $A^{2} \Theta-\Phi^{2}=2^{8} \Psi$ ) divides out by $2^{8} \Psi$, giving

$$
\Theta^{2}-2^{3} \cdot 3^{2} A^{2} \Theta \Phi+2^{6} \Phi^{3}+2^{12} \cdot 3^{3} A^{4} \Psi=0,
$$

or, what is the same thing,

$$
\Theta^{2}-2^{3} \cdot 3^{2} A^{2} \Theta \Phi+2^{6} \Phi^{3}+2^{4} \cdot 3^{3} A^{4}\left(A^{2} \Theta-\Phi^{2}\right)=0 .
$$

This may also be written in the form

$$
\left(\Theta-2^{2} \cdot 3^{2} A^{2} \Phi+2^{3} \cdot 3^{3} A^{6}\right)^{2}+2^{6}\left(\Phi-3^{2} A^{4}\right)^{3}=0,
$$

or, replacing $\Theta$ and $\Phi$ by their values in terms of $A, B, C, D$, $\left\{2^{6}\left(A^{2} D^{2}-6 A B C D+4 A C^{3}+4 B^{3} D+3 B^{2} C^{2}\right)\right.$

$$
\left.-2^{4} \cdot 3^{2} A^{2}\left(A^{2} D-3 A B C+2 B^{3}\right)-3^{3} A^{6}\right\}^{2}+2^{15}\left(A^{2} D-3 A B C+2 B^{3}\right)^{3}=0 \text {. }
$$

For a cubic whose invariant $S$ vanishes, the differential equation is

$$
\Theta^{2}-2^{12} \cdot 3 \Phi \Psi=0,
$$

and for a cubic whose invariant $T$ vanishes,

$$
\Theta^{3}-2^{11} \cdot 3^{2} \Theta \Phi \Psi+2^{21} \cdot 3^{3} A^{2} \Psi^{2}=0 .
$$

For the cuspidal cubic, both $S$ and $T$ vanish, so that the algebraic equation of the cuspidal cubic is a particular solution of each of these equations. We can, however, replace the system

$$
\begin{aligned}
& \Theta^{2}-2^{12} \cdot 3 \Phi \Psi=0, \\
& \Theta^{3}-2^{11} \cdot 3^{2} \Theta \Phi \Psi+2^{21} \cdot 3^{3} A^{2} \Psi^{2}=0,
\end{aligned}
$$

by another pair of equations, for one of which the cuspidal cubic is a particular solution, and for the other the complete primitive.

Multiplying the first equation by $\Theta$ and subtracting the second from it, we have, after dividing by $2^{11} \cdot 3 \Psi$,

$$
\Theta \Phi-2^{10} \cdot 3^{2} A^{2} \Psi=0 \text {. }
$$

From (1) and (3) we obtain

Hence

$$
\Theta^{2} \Phi^{2}=2^{12} \cdot 3 \Phi^{3} \Psi=2^{20} \cdot 3^{4} A^{4} \Psi^{2} .
$$

But

$$
\Phi^{3}=2^{8} \cdot 3^{3} A^{4} \Psi \text {. }
$$

so that

$$
A^{2} \Theta=\Phi^{2}+2^{8} \Psi
$$$$
A^{2} \Theta \Phi=\Phi^{3}+2^{8} \Phi \Psi \text {. }
$$

Substituting in this the values of $\Theta \Phi$ and $\Phi^{3}$ found from (3) and (4) and dividing by $\Psi$, we have

which gives

$$
\begin{aligned}
2^{10} \cdot 3^{2} A^{4} & =2^{8} \cdot 3^{3} A^{4}+2^{8} \Phi \\
\Phi & =3^{2} A^{4}
\end{aligned}
$$


Substituting this value of $\Phi$ in (4) and rejecting the factor $3^{3} A^{4}$, we obtain

$$
3^{3} A^{8}=2^{8} \Psi
$$

that is

$$
\left(\frac{A}{2}\right)^{8}=\left(\frac{A C-B^{2}}{3}\right)^{3}
$$

In the course of the work we have only rejected powers of $\Psi$ (that is of $A C-B^{2}$ ) and of $A$, of which neither corresponds to the cuspidal cubic.

Since $\Phi=3^{2} A^{4}$, it follows that $A^{2} D-3 A B C+2 B^{3}=0$. The equation to the cuspidal cubic above obtained is a particular solution of this, its complete primitive being (see Lecture XXXI. [above, p. 486]), $Y=X^{\lambda} Z^{1-\lambda}$, where $\lambda$ is an arbitrary constant.

\section{LECTURE XXXIV.}

The preceding 33 lectures contain the substance of the lectures on Reciprocants actually delivered, entire or in abstract, in the course of three terms, to a class at the University of Oxford.

A good deal of material remains over which the lecturer has lacked leisure or energy to throw into form, which he hopes to be able to recover and annex to what has gone before as supplemental matter in the convenient form of lectures numbered on from those which have already appeared.

The one that follows is entirely due to Mr Hammond, who has rendered invaluable aid in compiling, and in many cases bettering, the lectures previously published.

It constitutes probably the most difficult problem in elimination which has been effected up to the present time.

J. J. S.

The problem in question is to obtain the differential equation corresponding to the complete primitive

$$
\left(l^{\prime} x+m^{\prime} y+n^{\prime}\right)=(l x+m y+n)^{\lambda}\left(l^{\prime \prime} x+m^{\prime \prime} y+n^{\prime \prime}\right)^{1-\lambda}
$$

(say $Y=X^{\lambda} Z^{1-\lambda}$ ) by the process of eliminating all the arbitrary constants except $\lambda$.

The eliminations to be performed become greatly simplified by aid of the following Lemma. If $X$ be any linear function of $x$ and $y$, and $M_{a}$ the absolute pure reciprocant corresponding to $M$; then

$$
X_{3}-4 M_{a} X_{1}=0
$$

where

$$
\frac{d X}{d x}=a^{\frac{1}{3}} X_{1}, \quad \frac{d X_{1}}{d x}=a^{\frac{1}{3}} X_{2}, \quad \frac{d X_{2}}{d x}=a^{\frac{1}{3}} X_{3}
$$

For if we suppose

$$
X=l x+m y+n,
$$

two successive differentiations give

$$
a^{\frac{1}{3}} X_{1}=l+m t
$$

and

$$
a^{\frac{2}{3}} X_{2}+a^{-\frac{2}{3}} b X_{1}=2 m a \text {. }
$$


Writing the second of these equations in the form

$$
a^{-\frac{1}{3}} X_{2}+a^{-\frac{5}{3}} b X_{1}=2 m
$$

and differentiating again, we find

$$
X_{3}-a^{-\frac{4}{3}} b X_{2}+a^{-\frac{4}{3}} b X_{2}+\left(4 a c-5 b^{2}\right) a^{-\frac{8}{3}} X_{1}=0,
$$

or, since $4 M_{a}=\left(4 a c-5 b^{2}\right) a-\frac{8}{3}$,

$$
X_{3}+4 M_{a} X_{1}=0
$$

N.B.-Throughout the following work all letters with numerical suffixes are to be considered as derived from the corresponding unsuffixed letters in the same way as, in what precedes, $X_{1}, X_{2}$, and $X_{3}$ are derived from $X$; namely by successive differentiations, each of which is accompanied by a division by $a^{\frac{1}{3}}$.

Writing the equation

$$
Y=X^{\lambda} Z^{1-\lambda}
$$

(in which $X, Y, Z$ denote any three linear functions of $x, y$ ) in the form

$$
\log Y=\lambda \log X+(1-\lambda) \log Z,
$$

we obtain by differentiation and division by $a^{\frac{1}{3}}$,

$$
\frac{Y_{1}}{Y}=\lambda \frac{X_{1}}{X}+(1-\lambda) \frac{Z_{1}}{Z} \text {. }
$$

Let now

$$
\begin{aligned}
& X_{1}=u X, \\
& Y_{1}=v Y, \\
& Z_{1}=w Z,
\end{aligned}
$$

so that (1) takes the form

and consequently

$$
\begin{gathered}
v=\lambda u+(1-\lambda) w, \\
v_{1}=\lambda u_{1}+(1-\lambda) w_{1}, \\
v_{2}=\lambda u_{2}+(1-\lambda) w_{2} .
\end{gathered}
$$

By means of the Lemma it can be shown that

$$
\begin{gathered}
u^{3}+3 u u_{1}+u_{2}+4 M_{a} u=0 \\
v^{3}+3 v v_{1}+v_{2}+4 M_{a} v=0 \\
w^{3}+3 w w_{1}+w_{2}+4 M_{a} w=0 .
\end{gathered}
$$

For, since $\quad X_{1}=X u$,

$$
\begin{array}{ll}
\text { we have } & X_{2}=X_{1} u+X u_{1}=X\left(u^{2}+u_{1}\right) \\
\text { and } & X_{3}=X_{2} u+2 X_{1} u_{1}+X u_{2}=X\left(u^{3}+3 u u_{1}+u_{2}\right) .
\end{array}
$$

Substituting these values for $X_{1}$ and $X_{3}$ in

$$
X_{3}+4 M_{a} X_{1}=0 \text {, }
$$

we obtain

$$
u^{3}+3 u u_{1}+u_{2}+4 M_{a} u=0 \text {, }
$$


which proves equation (2). The equations (3) and (4) connecting $v, v_{1}, v_{2}$ and $w, w_{1}, w_{2}$ are similarly established. We now write

These, combined with

$$
\left.\begin{array}{c}
u+v+w=3 \omega \\
u-w=3 z
\end{array}\right\} .
$$

which, when operated on by $a^{-\frac{1}{3}} \frac{d}{d x}$ twice in succession, yield

$$
\left.\left.\begin{array}{c}
u_{1}=\omega_{1}-(\lambda-2) z_{1} \\
v_{1}=\omega_{1}-(1-2 \lambda) z_{1} \\
w_{1}=\omega_{1}-(\lambda+1) z_{1}
\end{array}\right\}, \quad \begin{array}{c}
u_{2}=\omega_{2}-(\lambda-2) z_{2} \\
v_{2}=\omega_{2}-(1-2 \lambda) z_{2} \\
w_{2}=\omega_{2}-(\lambda+1) z_{2}
\end{array}\right\} .
$$

When expressed in terms of $\omega, \omega_{1}, \omega_{2}$ and $z, z_{1}, z_{2}$, equations (2), (3), and (4) become transformed into

$$
\begin{aligned}
& P-(\lambda-2) Q+(\lambda-2)^{2} R-(\lambda-2)^{3} z^{3}=0 \\
& P-(1-2 \lambda) Q+(1-2 \lambda)^{2} R-(1-2 \lambda)^{3} z^{3}=0 \\
& P-(\lambda+1) Q+(\lambda+1)^{2} R-(\lambda+1)^{3} z^{3}=0
\end{aligned}
$$

where, for the sake of brevity, we write

$$
\begin{array}{r}
\omega^{3}+3 \omega \omega_{1}+\omega_{2}+4 M_{a} \omega=P, \\
3 \omega^{2} z+3 \omega z_{1}+3 \omega_{1} z+z_{2}+4 M_{a} z=Q \\
3 \omega z^{2}+3 z z_{1}=R .
\end{array}
$$

In order to simplify (5), (6), and (7), we multiply the first of them by $\lambda$, the second by -1 , and the third by $1-\lambda$, and take their sum, which is obviously independent of $P$, and from which it is easily seen that the terms containing $Q$ and $z^{3}$ will also disappear. For

$$
\lambda(\lambda-2)-(1-2 \lambda)+(1-\lambda)(\lambda+1)=0,
$$

and

$$
\lambda(\lambda-2)^{3}-(1-2 \lambda)^{3}+(1-\lambda)(\lambda+1)^{3}=0 \text {. }
$$

We are thus left with

$$
\left\{\lambda(\lambda-2)^{2}-(1-2 \lambda)^{2}+(1-\lambda)(\lambda+1)^{2}\right\} R=0,
$$

which, on restoring the value of $R$ and reducing, becomes

$$
\lambda(\lambda-1) z\left(\omega z+z_{1}\right)=0 \text {. }
$$

Now the values of $u, v, w$, which are equal to $\frac{X_{1}}{X}, \frac{Y_{1}}{Y}, \frac{Z_{1}}{Z}$ respectively, being distinct from each other, $z$ cannot vanish; for $z=0$ would imply $u=v=w$. Hence, considering $\lambda$ to have any finite numerical value except 1 or 0 , we may write

$$
\omega z+z_{1}=0
$$


in equations (5), (6), (7), which will then become

$$
\begin{aligned}
& P-(\lambda-2)\left(3 \omega_{1} z+z_{2}+4 M_{a} z\right)-(\lambda-2)^{3} z^{3}=0, \\
& P-(1-2 \lambda)\left(3 \omega_{1} z+z_{2}+4 M_{a} z\right)-(1-2 \lambda)^{3} z^{3}=0, \\
& P-(\lambda+1)\left(3 \omega_{1} z+z_{2}+4 M_{a} z\right)-(\lambda+1)^{3} z^{3}=0 .
\end{aligned}
$$

Adding these together, we find

$$
\begin{aligned}
3 P & =\left\{(\lambda-2)^{3}+(1-2 \lambda)^{3}+(\lambda+1)^{3}\right\} z^{3} \\
& =3(\lambda-2)(1-2 \lambda)(\lambda+1) z^{3} .
\end{aligned}
$$

Restoring the value of $P$, and writing for shortness

there results

$$
(\lambda-2)(\lambda+1)(2 \lambda-1)=p,
$$

From any pair of the equations (8), (9), (10) we obtain by subtraction

$$
3 \omega_{1} z+z_{2}+4 M_{a} z+3\left(\lambda^{2}-\lambda+1\right) z^{3}=0 .
$$

Thus, for example, subtracting (10) from (8), we have

$$
3\left(3 \omega_{1} z+z_{2}+4 M_{a} z\right)=\left\{(\lambda-2)^{3}-(\lambda+1)^{3}\right\} z^{3}=-9\left(\lambda^{2}-\lambda+1\right) z^{3} .
$$

Collecting our results, we see that equations (5), (6), (7) may be replaced by

$$
\begin{array}{r}
\omega^{3}+3 \omega \omega_{1}+\omega_{2}+4 M_{a} \omega+p z^{3}=0 \\
3 \omega_{1} z+z_{2}+4 M_{a} z+3 q z^{3}=0, \\
\omega z+z_{1}=0,
\end{array}
$$

where

$$
p=(\lambda-2)(\lambda+1)(2 \lambda-1) \text {, }
$$

and

$$
q=\lambda^{2}-\lambda+1 \text {. }
$$

Differentiating (13), we obtain

$$
\omega_{1} z+\omega z_{1}+z_{2}=0 \text {. }
$$

Subtracting this from (12) and adding (13) multiplied by $\omega$, the result divides by $z$, and we find

$$
\omega^{2}+2 \omega_{1}+4 M_{a}+3 q z^{2}=0,
$$

which, when multiplied by $\omega$ and subtracted from (11), reduces it to

$$
\omega \omega_{1}+\omega_{2}+p z^{3}-3 q z^{2} \omega=0 .
$$

Now it has been shown in Lecture XXX. [above, p. 482] that

$$
\begin{aligned}
& a^{-\frac{1}{3}} \frac{d}{d x} M_{a}=5 A_{a}, \\
& a^{-\frac{1}{3}} \frac{d}{d x} A_{a}=6 B_{a}, \\
& a^{-\frac{1}{3}} \frac{d}{d x} B_{a}=7 C_{a}+M_{a} A_{a},
\end{aligned}
$$

whence it follows that (14) gives on differentiation

$$
\omega \omega_{1}+\omega_{2}+10 A_{a}+3 q z z_{1}=0 .
$$


Combining this with (15) we have

or, finally, since $\omega z+z_{1}=0$,

$$
10 A_{a}=p z^{3}-3 q z\left(\omega z+z_{1}\right)
$$

Differentiating this, we have

$$
10 A_{a}=p z^{3}
$$

that is

$$
\begin{gathered}
20 B_{a}=p z^{2} z_{1}=-p z^{3} \omega \\
2 B_{a}+A_{a} \omega=0,
\end{gathered}
$$

whence, by differentiation,

$$
14 C_{a}+2 M_{a} A_{a}+6 B_{a} \omega+A_{a} \omega_{1}=0 .
$$

Subtracting (14) multiplied by $A_{a}$ from the double of this, we have

$$
28 C_{a}-A_{a} \omega^{2}+12 B_{a} \omega-3 q z^{2} A_{a}=0 .
$$

Substituting in this for $\omega$ its value $-\frac{2 B_{a}}{A_{a}}$, found from (16), there results

But it has been shown that

$$
28\left(A_{a} C_{a}-B_{a}^{2}\right)=3 q z^{2} A_{a}^{2} .
$$

Hence the elimination of $z$ gives

$$
10 A_{a}=p z^{3}
$$

$$
28^{3} p^{2}\left(A_{a} C_{a}-B_{a}{ }^{2}\right)^{3}=3^{3} q^{3} p^{2} z^{6} A_{a}{ }^{6}=10^{2} 3^{3} q^{3} A_{a^{8}} .
$$

Or restoring for $p$ and $q$ their values in terms of $\lambda$, and replacing the absolute reciprocants $A_{a}, B_{a}, C_{a}$ by the non-absolute ones $A, B, C$ (which is effected by merely multiplying throughout by a power of $a$ ), we have

$$
2^{4} \cdot 7^{3}(\lambda-2)^{2}(\lambda+1)^{2}(2 \lambda-1)^{2}\left(A C-B^{2}\right)^{3}=3^{3} \cdot 5^{2}\left(\lambda^{2}-\lambda+1\right)^{3} A^{8} .
$$

For other methods of obtaining this differential equation see Halphen's Thèse sur les Invariants Différentiels, p. 30, and Lecture XXX. of the present course. It corresponds in general (that is unless $\lambda=0,1, \infty$ ) to the complete primitive

$$
Y=X^{\lambda} Z^{1-\lambda}
$$

When $\lambda=0,1, \infty$, the differential equation (17) becomes

$$
28^{3}\left(A C-B^{2}\right)^{3}=3^{3} .5^{2} A^{8}
$$

which corresponds to the complete primitive

$$
Y=X e^{\frac{Z}{X}}
$$

This case has been discussed in the Thèse and in Lecture XXX. [above, p. 480$]$.

We may obtain (18) from (19) by a method of elimination similar to that employed in deducing (17) from its complete primitive. Thus the first differential of (19) may be written

$$
\frac{Y_{1}}{Y}=\frac{X_{1}}{X}+\frac{Z_{1} X-Z X_{1}}{X^{2}}
$$

which becomes

$$
v=u+3 z
$$

when we assume

$$
X_{1}=X u, \quad Y_{1}=Y v, \quad Z_{1}=Z u+3 X_{z} .
$$


By means of the Lemma we obtain

$$
\begin{array}{r}
u^{3}+3 u u_{1}+u_{2}+4 M_{a} u=0, \\
v^{3}+3 v v_{1}+v_{2}+4 M_{a} v=0 \\
3 u^{2} z+3 u_{1} z+3 u z_{1}+z_{2}+4 M_{a} z=0 .
\end{array}
$$

The first two of these are identical with (2) and (3) previously given; the third is found as follows. Since

$$
\begin{aligned}
Z_{1} & =Z u+3 X z, \\
Z_{2} & =Z_{1} u+Z u_{1}+3 X_{1} z+3 X_{z_{1}} \\
& =Z\left(u^{2}+u_{1}\right)+3 X\left(2 u z+z_{1}\right) .
\end{aligned}
$$

Hence

$$
\begin{aligned}
Z_{3} & =Z_{1}\left(u^{2}+u_{1}\right)+Z\left(2 u u_{1}+u_{2}\right)+3 X_{1}\left(2 u z+z_{1}\right)+3 X\left(2 u_{1} z+2 u z_{1}+z_{2}\right) \\
& =Z\left(u^{3}+3 u u_{1}+u_{2}\right)+3 X\left(3 u^{2} z+3 u_{1} z+3 u z_{1}+z_{2}\right) .
\end{aligned}
$$

Thus we have

$$
Z_{3}+4 M_{a} Z_{1}=Z\left(u^{3}+3 u u_{1}+u_{2}+4 M_{a} u\right)+3 X\left(3 u^{2} z+3 u_{1} z+3 u z_{1}+z_{2}+4 M_{a} z\right) \text {. }
$$

But $Z_{3}+4 M_{a} Z_{1}=0$, and $u^{3}+3 u u_{1}+u_{2}+4 M_{a} u=0$, which shows that

$$
3 u^{2} z+3 u_{1} z+3 u z_{1}+z_{2}+4 M_{a} z=0 .
$$

Equations (20), (21), and (22), of which we have just proved the last, are merely convenient expressions of the fact that $X, Y, Z$ are linear functions of $x, y$. We combine them with the first, second, and third differentials of the primitive equation (19) by writing

$$
\left.\begin{array}{c}
v=u+3 z \\
v_{1}=u_{1}+3 z_{1} \\
v_{2}=u_{2}+3 z_{2}
\end{array}\right\} .
$$

When this is done (21) becomes

$$
\begin{aligned}
\left(u^{3}+3 u u_{1}+u_{2}+4 M_{a} u\right)+3\left(3 u^{2} z+3 u z_{1}+3 u_{1} z\right. & \left.+z_{2}+4 M_{a} z\right) \\
& +27 z\left(u z+z^{2}+z_{1}\right)=0,
\end{aligned}
$$

which, in consequence of the identities (20) and (22), reduces to

$$
(u+z) z+z_{1}=0 \text {. }
$$

Let now $u=\omega-z$ (so that $\omega z+z_{1}=0$ ). Substituting in (20) and (22) we find

$$
\omega^{3}+3 \omega \omega_{1}+\omega_{2}+4 M_{a} \omega-3(\omega-z)\left(\omega z+z_{1}\right)-z^{3}-3 \omega_{1} z-z_{2}-4 M_{a} z=0,
$$

and $\quad(3 \omega-6 z)\left(\omega z+z_{1}\right)+3 z^{3}+3 \omega_{1} z+z_{2}+4 M_{a} z=0$

respectively. Adding both equations together, and remembering that

$$
\omega z+z_{1}=0
$$

we obtain

$$
\begin{array}{r}
\omega^{3}+3 \omega \omega_{1}+\omega_{2}+4 M_{a} \omega+2 z^{3}=0, \\
3 \omega_{1} z+z_{2}+4 M_{a} z+3 z^{3}=0,
\end{array}
$$

which, combined with

$$
\omega z+z_{1}=0
$$

replace the system (20), (21), (22). 
Comparing these equations with (11), (12), (13), we see that the two sets are identical if we make $\lambda=0$, when $p$ becomes 2 and $q=1$. Hence, by performing exactly the same work as in the previous case, we shall find

$$
5 A_{a}=z^{3} \quad\left(\text { instead of } 10 A_{a}=p z^{3}\right)
$$

and

$$
\left.28\left(A_{a} C_{a}-B_{a}^{2}\right)=3 z^{2} A_{a}^{2} \text { (instead of } 3 q z^{2} A_{a}^{2}\right) \text {. }
$$

And, finally, eliminating $z$ between this pair of equations, at the same time replacing the absolute reciprocants $A_{a}, B_{a}, C_{a}$ by the corresponding non-absolute ones $A, B, C$, we have

$$
28^{3}\left(A C-B^{2}\right)^{3}=3^{3} .5^{2} A^{8}
$$

which is what (17) becomes when $\lambda$ has any of the values 0,1 , or $\infty$. 